\title{
WestVirginiaUniversity
}

THE RESEARCH REPOSITORY @ WVU

Graduate Theses, Dissertations, and Problem Reports

2014

\section{The Modeling and Advanced Controller Design of Wind, PV and Battery Inverters}

Junbiao Han

West Virginia University

Follow this and additional works at: https://researchrepository.wvu.edu/etd

\section{Recommended Citation}

Han, Junbiao, "The Modeling and Advanced Controller Design of Wind, PV and Battery Inverters" (2014). Graduate Theses, Dissertations, and Problem Reports. 121.

https://researchrepository.wvu.edu/etd/121

This Dissertation is protected by copyright and/or related rights. It has been brought to you by the The Research Repository @ WVU with permission from the rights-holder(s). You are free to use this Dissertation in any way that is permitted by the copyright and related rights legislation that applies to your use. For other uses you must obtain permission from the rights-holder(s) directly, unless additional rights are indicated by a Creative Commons license in the record and/ or on the work itself. This Dissertation has been accepted for inclusion in WVU Graduate Theses, Dissertations, and Problem Reports collection by an authorized administrator of The Research Repository @ WVU.

For more information, please contact researchrepository@mail.wvu.edu. 


\title{
The Modeling and Advanced Controller Design of Wind, PV and Battery Inverters
}

\author{
Junbiao Han
}

\author{
Dissertation submitted \\ to the Benjamin M. Statler College of Engineering and Mineral Resources \\ at West Virginia University \\ In partial fulfillment of the requirements for the degree of \\ Doctor of Philosophy in \\ Electrical Engineering
}

Prof. Sarika Khushalani-Solanki, Ph.D., Chair

Prof. Muhammad A. Choudhry, Ph.D.

Prof. Ali Feliachi, Ph.D.,

Prof. Jignesh Solanki, Ph.D.

Prof. Qipeng phil Zheng, Ph.D.

LANE DEPARTMENT OF COMPUTER SCIENCE AND ELECTRICAL ENGINEERING

Morgantown, West Virginia

2014

Keywords: Microgrid, PhotoVoltaic, Wind Farm, Battery, Coordination control, Autonomous control, Genetic Algorithm, Dual Heuristic Programming, EMTDC/PSCAD, RTDS

Copyright 2014 Junbiao Han 


\title{
Abstract
}

The Modeling and Advanced Controller Design of Wind, PV and Battery Inverters

\author{
Junbiao Han
}

Renewable energies such as wind power and solar energy have become alternatives to fossil energy due to the improved energy security and sustainability. This trend leads to the rapid growth of wind and Photovoltaic (PV) farm installations worldwide. Power electronic equipments are commonly employed to interface the renewable energy generation with the grid. The intermittent nature of renewable and the large scale utilization of power electronic devices bring forth numerous challenges to system operation and design. Methods for studying and improving the operation of the interconnection of renewable energy such as wind and $\mathrm{PV}$ are proposed in this $\mathrm{Ph} . \mathrm{D}$. dissertation.

A multi-objective controller including is proposed for PV inverter to perform voltage flicker suppression, harmonic reduction and unbalance compensation. A novel supervisory control scheme is designed to coordinate PV and battery inverters to provide high quality power to the grid. This proposed control scheme provides a comprehensive solution to both active and reactive power issues caused by the intermittency of PV energy. A novel real-time experimental method for connecting physical PV panel and battery storage is proposed, and the proposed coordinated controller is tested in a Hardware in the Loop (HIL) experimental platform based on Real Time Digital Simulator (RTDS).

This work also explores the operation and controller design of a microgrid consisting of a direct drive wind generator and a battery storage system. A Model Predictive Control (MPC) strategy for the AC-DC-AC converter of wind system is derived and implemented to capture the maximum wind energy as well as provide desired reactive power. The MPC increases the accuracy of maximum wind energy capture as well as minimizes the power oscillations caused by varying wind speed. An advanced supervisory controller is presented and employed to ensure the power balance while regulating the PCC bus voltage within acceptable range in both grid-connected and islanded operation.

The high variability and uncertainty of renewable energies introduces unexpected fast power variation and hence the operation conditions continuously change in distribution networks. A three-layers advanced optimization and intelligent control algorithm for a microgrid with multiple renewable resources is proposed. A Dual Heuristic Programming (DHP) based system control layer is used to ensure the dynamic reliability and voltage stability of the entire microgrid as the system operation condition changes. A local layer maximizes the capability of the Photovoltaic (PV), wind power generators and battery systems, and a Model Predictive Control (MPC) based device layer increases the tracking accuracy of the converter control. The detail design of the proposed SWAPSC scheme are presented and tested on an IEEE 13 node feeder with a PV farm, a wind farm and two battery-based energy storage systems. 


\section{Acknowledgements}

Many people have inspired, encouraged and supported me in every way during my study at West Virginia University. Without whom, it would not have been possible for me to finish this dissertation.

Dr. Sarika Khushalani-Solanki has been a wise and trustworthy advisor to me throughout my entire study. It is due to her constant support and encouragement that I have gained a deeper understanding of electric engineering and made progress toward solving problems and improving my writing and presentation skills as a researcher. Her vision, patience and her confidence in my ability have made this process a much easier one for me.

I have the greatest respect and gratitude to my co-advisor Dr. Jignesh Solanki for his understanding and invaluable input into my research. I would like to thank Dr. Ali Feliachi for his help and technical advices throughout my Ph.D. study. Dr. Muhammad A. Choudhry is the graduate program director in the power group who has inspired me so much and brought to me different angles of power engineering. I would also like to thank Dr. Qipeng Zheng for serving on my comprehensive exam and defense committee and sharing his knowledge and experience.

I would like to express my gratitude to all my friends and student colleagues who have supported me directly or indirectly during my study at West Virginia University. I was fortunate to work with them and they have been an enormous source of energy, support, and inspiration.

I would like to thank my parents, my brother and my sister for being my source of motivation and gave me my strength and confidence in my pursuit for the doctoral degree.

Most of all, I would like to thank my wife, Lang Chen, who has always been there for me, given me love and encouragement during the most difficult time, and come along with me in every step through the entire journey. 
ABSTRACT

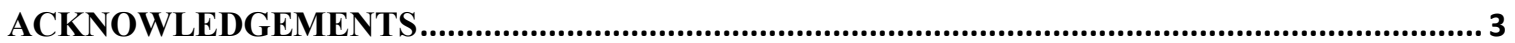

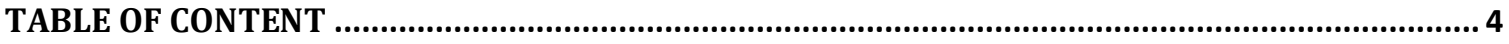

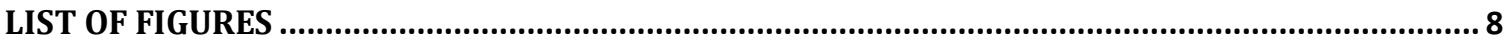

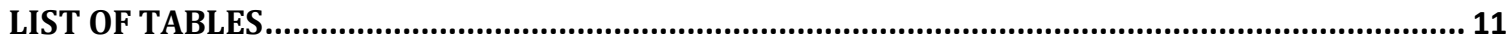

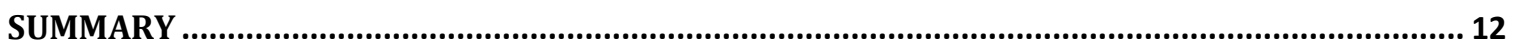

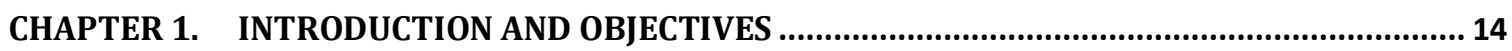

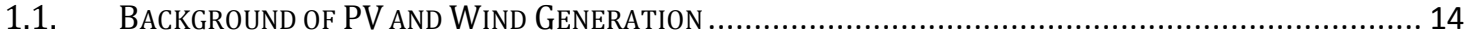

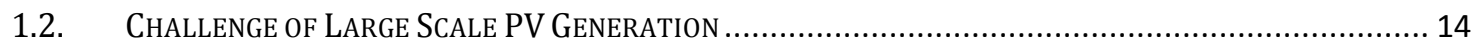

1.3. OPERATION OF Wind GENERATION IN GRID-CONNECTED AND ISLANDED MOdE .............................. 16

1.4. Optimal Operation of a Microgrid With High Penetration LeVel of Wind and PV

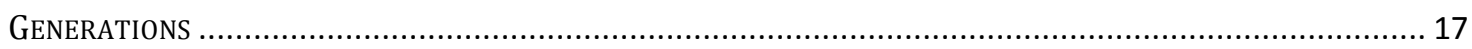

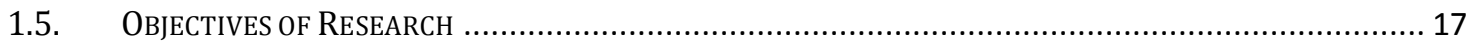

CHAPTER 2. LITERATURE REVIEW .............................................................................................. 19

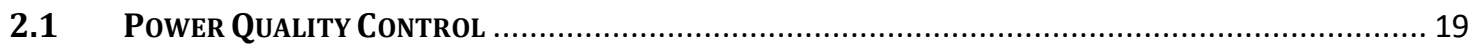

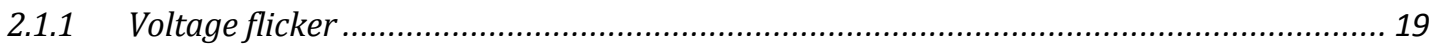

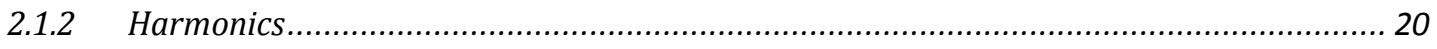

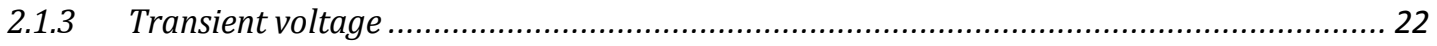

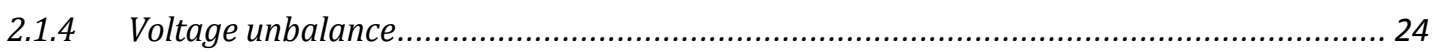

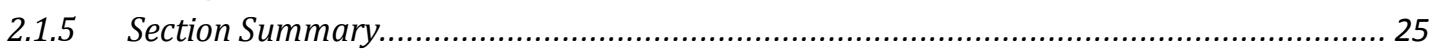

2.2 Microgrid Modeling ANd Coordination Controller DeSign .......................................... 26

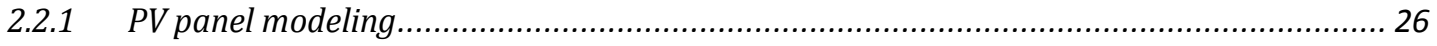

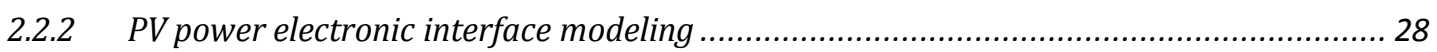

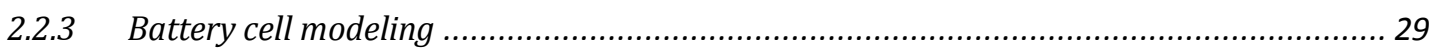

2.2.4 Battery power electronic interface modeling ............................................................ 30

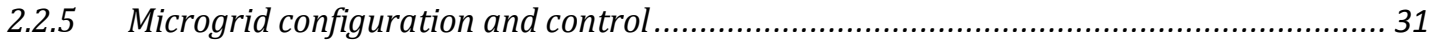

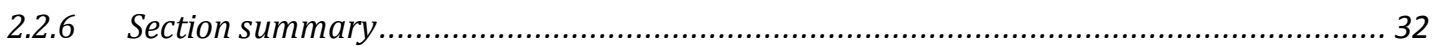

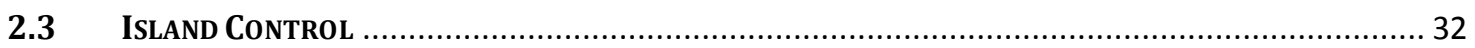

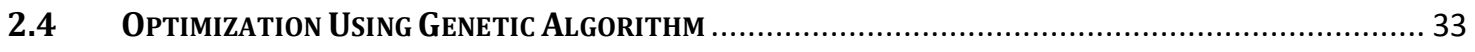

2.4.1. Crossover

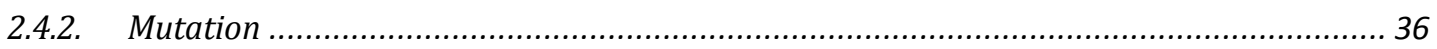

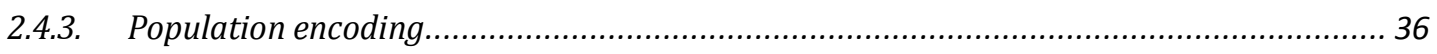

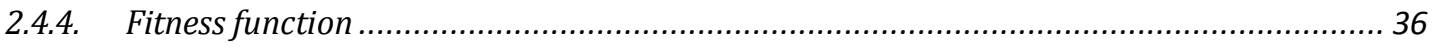

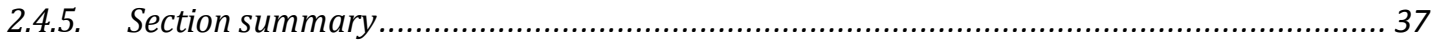

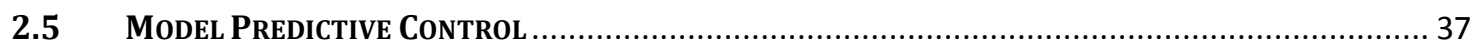

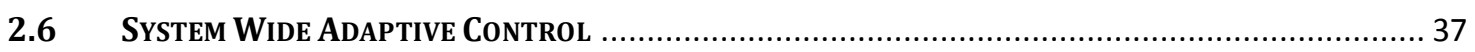

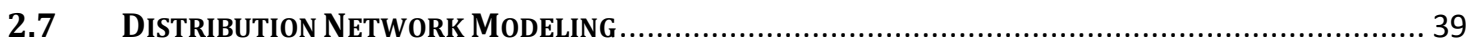

CHAPTER 3. PROBLEM STATEMENT …........................................................................................... 41

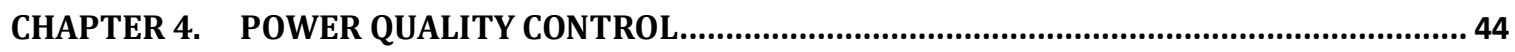




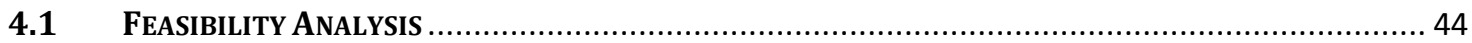

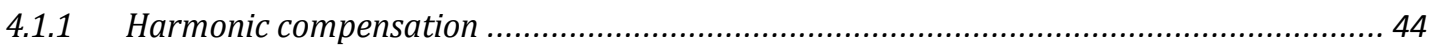

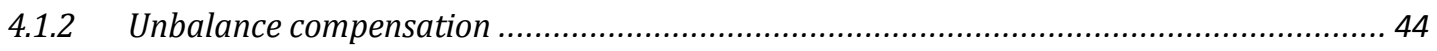

4.1.3 Voltage flicker suppression and Dynamic reactive power support................................. 45

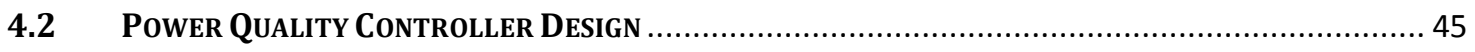

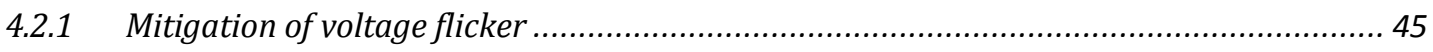

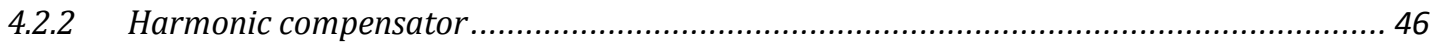

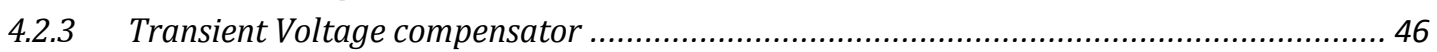

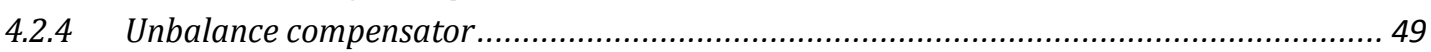

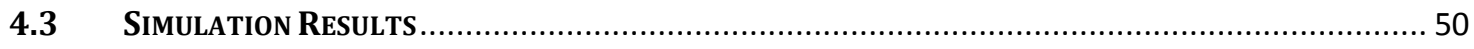

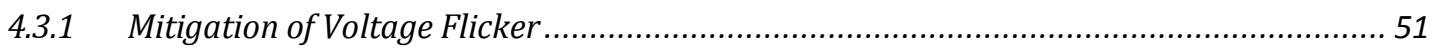

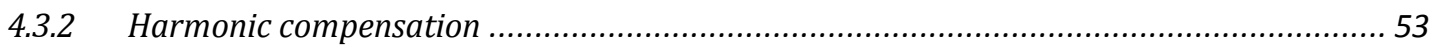

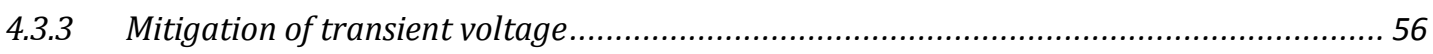

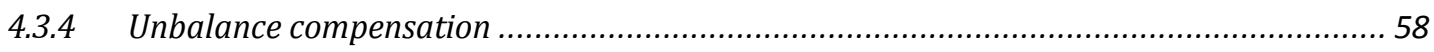

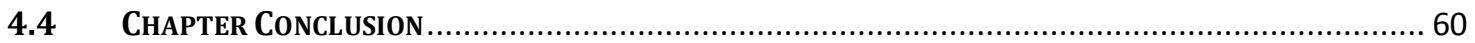

\section{CHAPTER 5. MODELING AND COORDINATED CONTROLLER DESIGN OF A PV/BATTERY SYSTEM 61}

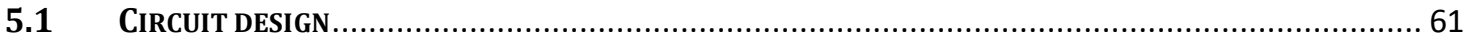

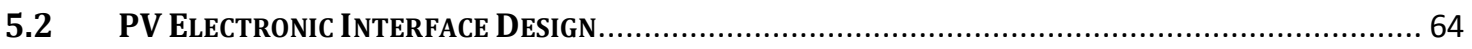

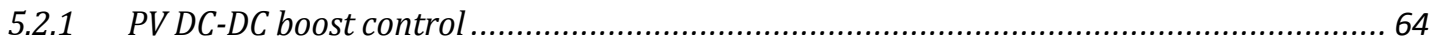

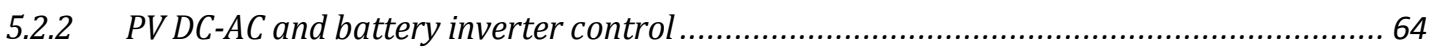

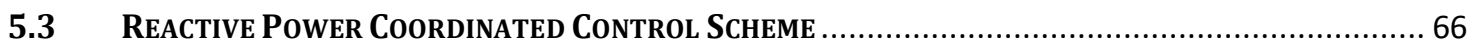

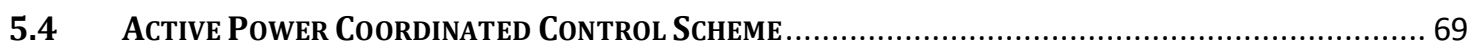

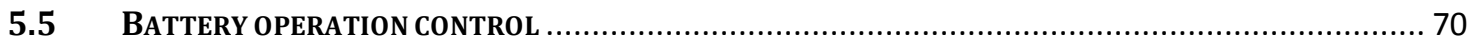

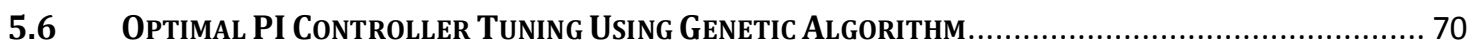

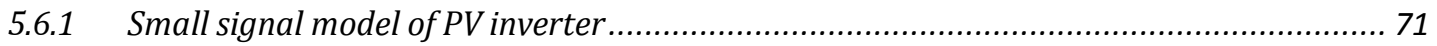

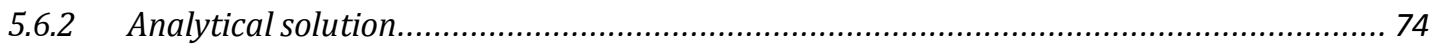

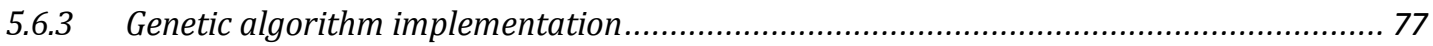

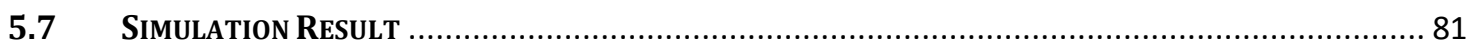

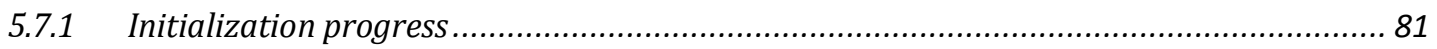

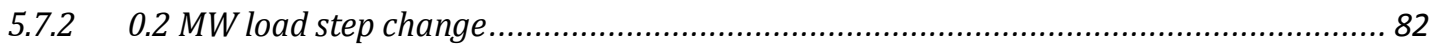

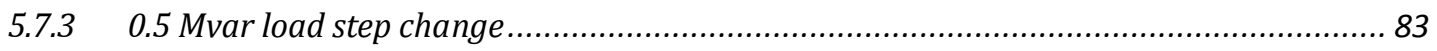

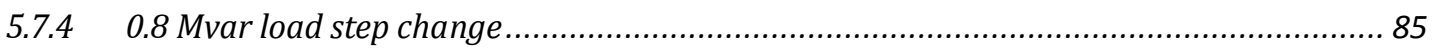

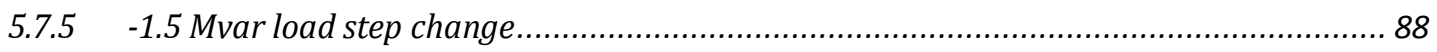

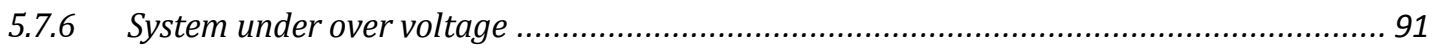

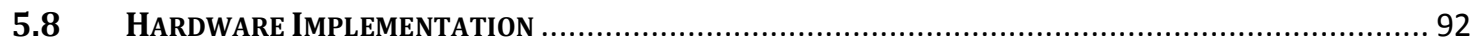

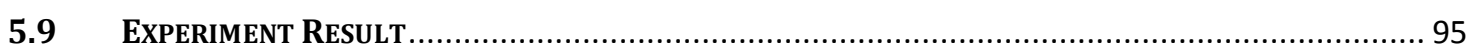

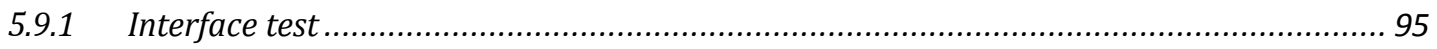

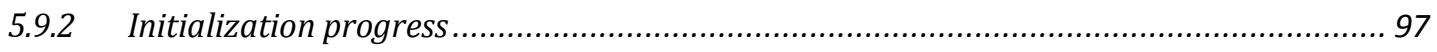

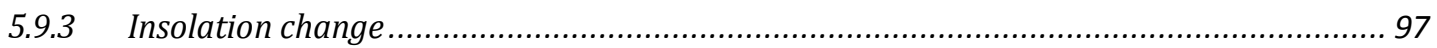

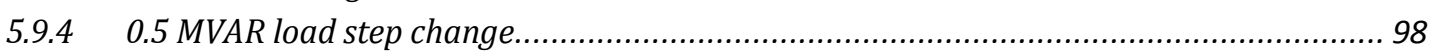

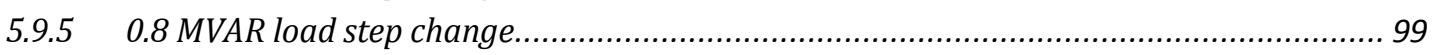

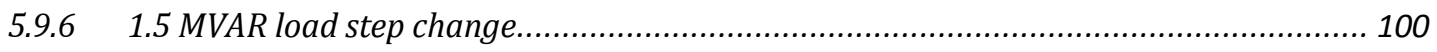

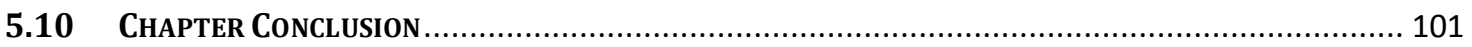




\section{CHAPTER 6. COORDINATED PREDICTIVE CONTROL OF A WIND/BATTERY MICROGRID SYSTEM 103}

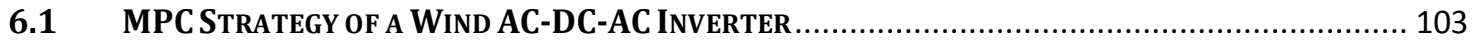

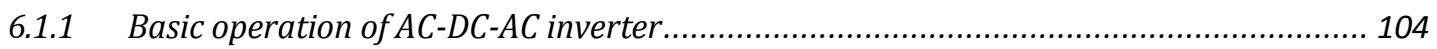

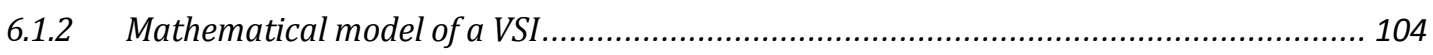

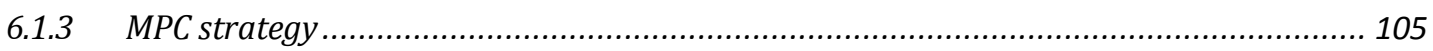

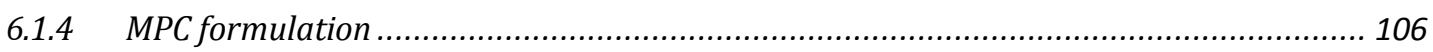

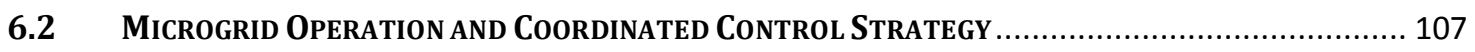

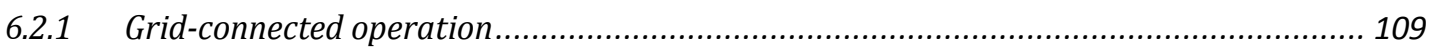

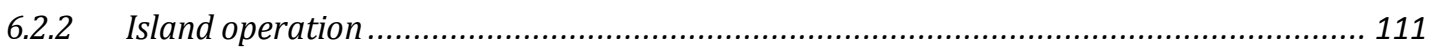

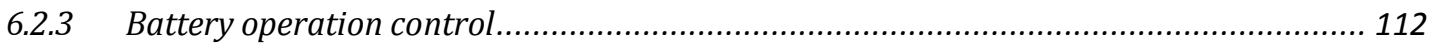

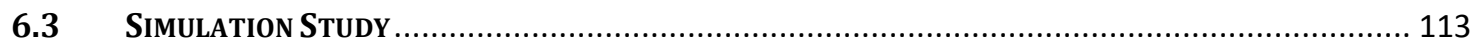

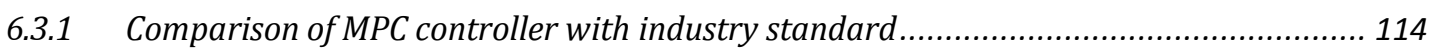

6.3.2 Reactive power coordination under grid-connected operation....................................... 115

6.3.3 Active power coordination under grid-connected operation ...................................... 117

6.3.4 Active and reactive power coordination under transition from grid-connected to islanded

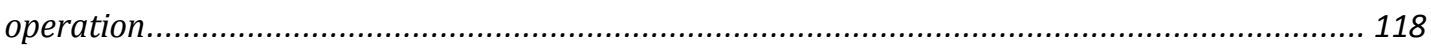

6.3.5 Active and reactive power coordination under transition from islanded to grid-connected

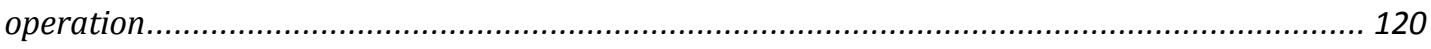

6.3.6 Reactive and active power coordination under black start in islanded operation ........... 120

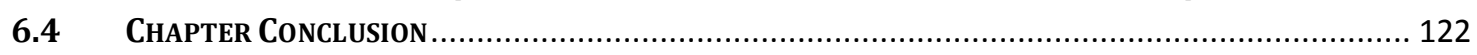

CHAPTER 7. ADAPTIVE CRITIC DESIGN BASED DYNAMIC STOCHASTIC OPTIMAL CONTROL DESIGN FOR A MICROGRID WITH MULTIPLE RENEWABLE RESOURCES................................... 123

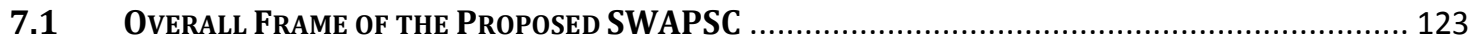

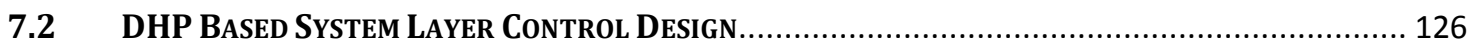

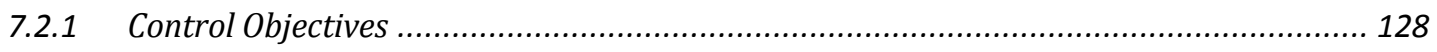

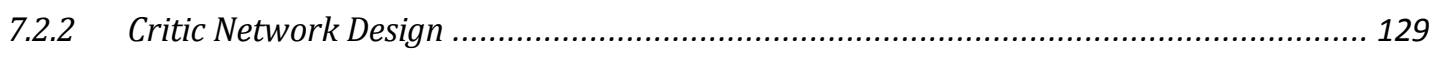

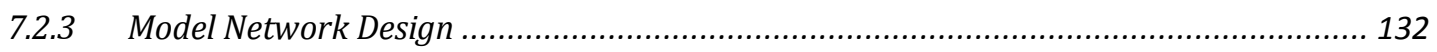

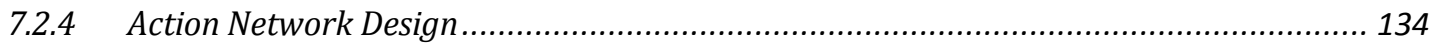

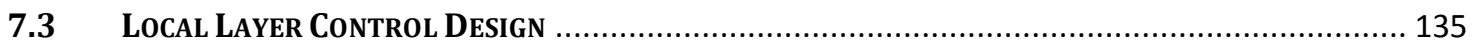

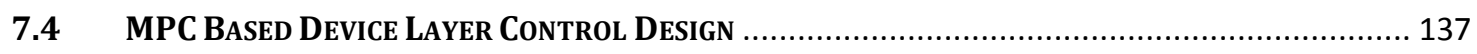

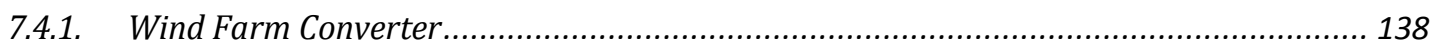

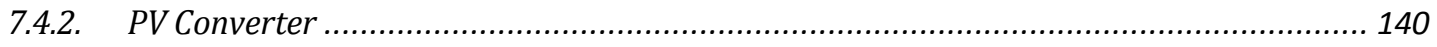

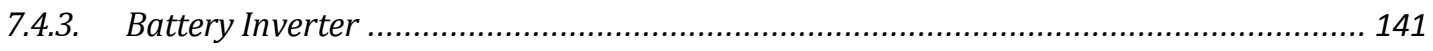

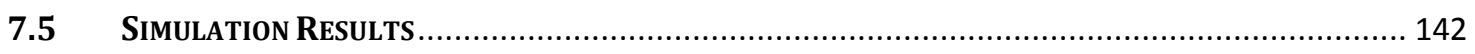

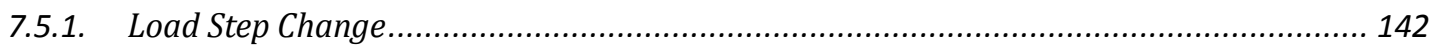

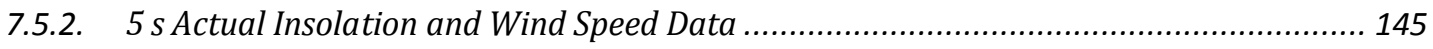

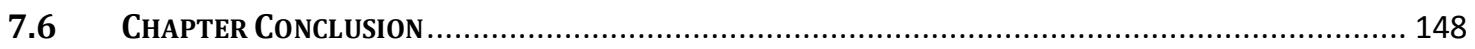

CHAPTER 8. SUMMARY CONCLUSION AND CONTRIBUTIONS.................................................. 150

CHAPTER 9. RECOMMENDATIONS FOR FUTURE WORK ......................................................... 153

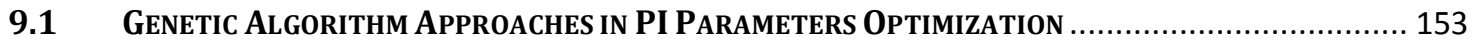

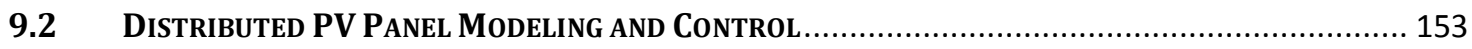

9.3 SWAPSC in REgional Voltage STABILITY IMPROVEMENT AND LOAD FREQUENCY CONTROL .... 153 


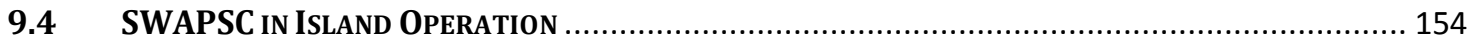

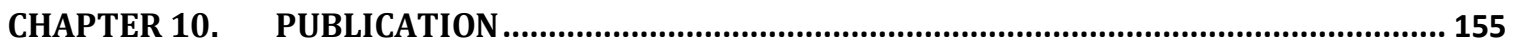

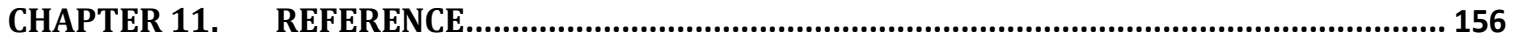

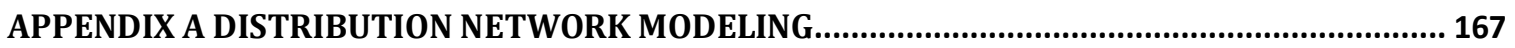

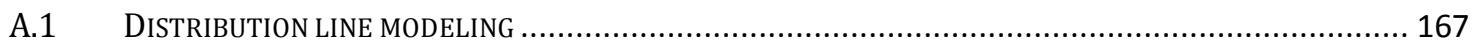

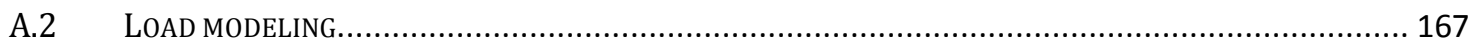

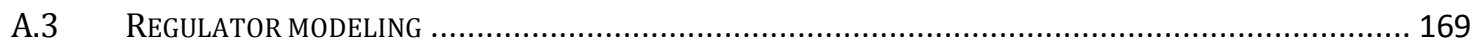

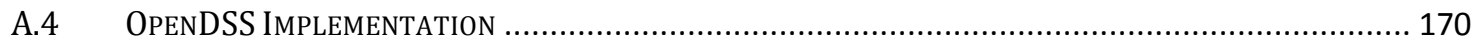

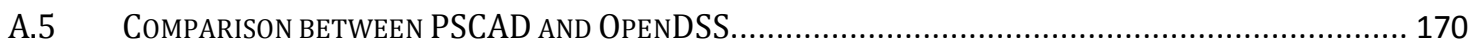

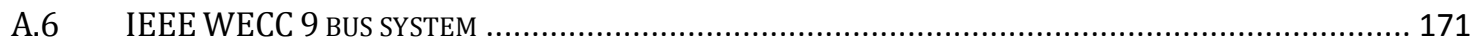




\section{List of Figures}

FIG.2.1 A VOLTAGE SOURCE INVERTER 20

FIG.2.2 UNBALANCE COMPENSATION 24

FIG.2.3 AN EQUIVALENT PV CELL CIRCUIT

FIG.2.4 PV GENERATOR MODEL IN PSCAD 27

FIG.2.5 EQUIVALENT CIRCUIT MODEL OF A BATTERY CELL 29

FIG.2.6 BUCK- BOOST CIRCUIT OF BSS

FIG.2.8 THE IDEA OF GENETIC ALGORITHM

FIG.2.9 IDEA OF CROSSOVER 36

FIG.2.10 IDEA OF MUTATION 36

FIG. 2.11 SINGLE LINE DIAGRAM OF IEEE 13 BUS FEEDER 39

FIG.4.1 FLICKER REDUCTION CONTROL OF PV INVERTER 46

FIG.4.2 HARMONIC REDUCTION CONTROL

FIG.4.3 INTERNAL CONTROL STRATEGY FOR TRANSIENT VOLTAGE REGULATION 48

FIG.4.4 FAULT RIDE THROUGH CONTROL

FIG.4.5 UNBALANCE COMPENSATION CONTROL

FIG.4.6 IEEE 34 NODES SYSTEM WITH PV INTEGRATION

FIG.4.7 INPUT IRRADIATION OF PV CELL

FIG.4.8 PV OUTPUT POWER TO THE GRID

FIG.4.9 GRID SIDE LINE VOLTAGE RMS VALUE

FIG.4.10 VOLTAGE AT THE TERMINALS OF THE FILTER $\quad 54$

FIG.4.11 PV PANEL CHARACTERISTICS WITH MPPT

FIG.4.12 THD OF VOLTAGE AND CURRENT WAVEFORM OF PV FARM

FIG.4.13 OUTPUT POWER OF PV FARM DURING HARMONIC COMPENSATION

FIG.4.14 PV GENERATION AND PCC BUS VOLTAGE

FIG.4.15 PCC BUS VOLTAGE RMS VALUE

FIG.4.16 DC CAPACITOR BANK VOLTAGE

FIG.4.17 THE TERMINAL VOLTAGE OF PV GENERATOR

FIG.4.18 UNBALANCED INDICES OF PCC VOLTAGE AND CURRENT

FIG.4.19 OUTPUT POWER OF PV FARM DURING UNBALANCE COMPENSATION 59

FIG.5.1 ONE LINE DIAGRAM OF THE HYBRID SYSTEM 61

FIG.5.2 POWER ELECTRONICS INTERFACE OF PV FARM

FIG.5.3 GAIN VERSUS FREQUENCY CURVE OF A SECOND-ORDER LC LOW- PASS FILTER 63

FIG.5.4 PV DC-DC BOOSTER CONTROLLER 64

FIG.5.5 PV INVERTER CONTROLLER

FIG.5.6 BATTERY INVERTER CONTROLLER 66

FIG.5.7 THE FLOW CHART OF REACTIVE POWER COORDINATION CONTROL SCHEME

FIG.5.8 FLOW CHART OF REACTIVE POWER COORDINATION FOR RTDS IMPLEMENTATION 69

FIG.5.9 CIRCUIT DIAGRAM OF A PV DC-AC INVERTER

FIG.5.10 AVERAGE PV INVERTER MODEL

FIG.5.11 CONTROL BLOCK OF A GRID CONNECTED INVERTER FOR PV SYSTEM 75

FIG.5.12 OPTIMIZATION PROCEDURES USING GA $\quad 77$

FIG.5.13 THE STRUCTURE OF THE CHROMOSOME

FIG.5.14 SCREENSHOT OF THE SIMULINK MODEL OF A GRID CONNECTED PV SYSTEM 79

FIG.5.15 FITNESS EVALUATION DIAGRAM 80 
FIG.5.16 ROULETTE WHEEL SELECTION ALGORITHM 80

FIG.5.17 SIMULATION RESULTS OF THE HYBRID SYSTEM UNDER INITIALIZATION MODE 82

FIG.5.18 SIMULATION RESULTS OF THE HYBRID SYSTEM UNDER LOAD STEP CHANGE FROM 0.77

MW TO 0.57 MW 83

FIG.5.19 SIMULATION RESULTS OF THE HYBRID SYSTEM UNDER 0.5 MVAR LOAD STEP CHANGE 84 FIG.5.20 SIMULATION RESULTS OF THE HYBRID SYSTEM WHEN THE LOAD CHANGES BACK FROM

0.5 MVAR TO 0 MVAR 85

FIG.5.21 SIMULATION RESULTS OF THE HYBRID SYSTEM UNDER 0.8 MVAR LOAD STEP CHANGE 86

FIG.5.22 SIMULATION RESULTS OF THE HYBRID SYSTEM WHEN THE LOAD CHANGES BACK FROM

0.8 MVAR TO 0 MVAR

FIG.5.23 SIMULATION RESULTS OF THE HYBRID SYSTEM UNDER -1.5 MVAR LOAD STEP CHANGE 88

FIG.5.24 SIMULATION RESULTS OF THE HYBRID SYSTEM WHEN THE LOAD CHANGE BACK FROM -

1.5 MVAR TO 0 MVAR

FIG.5.25 SIMULATION RESULT OF THE HYBRID SYSTEM UNDER OVER VOLTAGE

FIG.5.26 ONE LINE DIAGRAM OF THE HYBRID SYSTEM 93

FIG.5.27 HARDWARE SETUP OF THE MICROGRID

FIG.5.28 HARDWARE INTERFACE BOARD SETUP A) PV INTERFACE; B) BATTERY INTERFACE 95

FIG.5.29 INTERFACE TEST DURING INSOLATION CHANGE 96

FIG.5.30 SYSTEM PERFORMANCE DURING INITIALIZATION PROGRESS 97

FIG.5.31 SYSTEM PERFORMANCE DURING INSOLATION CHANGE 98

FIG.5.32 SYSTEM PERFORMANCE UNDER 0.5MVAR LOAD STEP CHANGE 99

FIG.5.33 SYSTEM PERFORMANCE DURING 0.8MVAR LOAD STEP CHANGE 100

FIG.5.34 SYSTEM PERFORMANCE UNDER -1.5MVAR LOAD STEP CHANGE 101

FIG.6.1 ONE LINE DIAGRAM OF THE MICROGRID 103

FIG.6.2 CIRCUIT DIAGRAM OF WIND CONVERTER 104

FIG.6.3 CONTROL SCHEME OF WIND CONVERTER 106

FIG.6.4 CONTROL SCHEME OF BATTERY VSI 107

FIG.6.5 COORDINATED CONTROL SCHEME OF THE MICROGRID WITH WIND AND BATTERY

$\begin{array}{ll}\text { STORAGE } & 108\end{array}$

FIG.6.6 ACTIVE POWER COORDINATION 111

FIG.6.7 ISLANDED CONTROL STRATEGY 112

FIG.6.8 COMPARISON BETWEEN MPC AND OPTIMIZED PI CONTROLLER 114

FIG.6.9 SYSTEM PERFORMANCE UNDER LOW VOLTAGE IN GRID-CONNECTED MODE 115

FIG.6.10 SYSTEM PERFORMANCE UNDER HIGH VOLTAGE IN GRID-CONNECTED MODE 116

FIG.6.11 SYSTEM PERFORMANCE UNDER WIND SPEED VARIATION 117

FIG.6.12 PERFORMANCE OF THE GENERATOR AND GRID SIDE MPC 118

FIG.6.13 SYSTEM RESPONSE TO OPERATION MODE CHANGE 119

FIG.6.14 SYSTEM PERFORMANCE DURING THE TRANSITION FROM GRID-CONNECTED TO

ISLANDED MODE 119

FIG.6.15 SYSTEM RESPONSE DURING THE TRANSITION FROM GRID-CONNECTED TO ISLANDED

MODE 120

FIG.6.16 SYSTEM PERFORMANCE DURING THE TRANSITION FROM GRID-CONNECTED TO

ISLANDED MODE $\quad 121$

FIG.6.17 SYSTEM PERFORMANCE DURING BLACK START IN ISLANDED OPERATION 122

FIG.7.1 IEEE 13 NODE SYSTEM WITH RENEWABLE RESOURCES. 124

FIG.7.2 STRUCTURE OF THE PROPOSED SWAPSC. $\quad 125$

FIG.7.3 SYSTEM LAYER CONTROL USING DHP 127

FIG.7.4 THE STRUCTURE OF CRITIC NETWORK 131 
FIG.7.5 THE STRUCTURE OF MODEL NETWORK

FIG.7.6 THE STRUCTURE OF ACTION NETWORK

FIG.7.7 LOCAL CONTROL LAYER CONFIGURATION OF THE WIND SET 136

FIG.7.8 OVERALL CONTROL OF WIND FARM $\quad 138$

FIG.7.9 OVERALL CONTROL OF PV FARM $\quad 141$

FIG.7.10 OVERALL CONTROL OF BATTERY

FIG.7.11 PCC BUS VOLTAGES WITH SWAPSC UNDER LOAD STEP CHANGE 143

FIG.7.12 SMOOTHING OF PV AND WIND FARM POWER WITH BESS UNDER LOAD STEP CHANGE 144

FIG.7.13 BESS PERFORMANCE WITH SOC CONTROL UNDER LOAD STEP CHANGE

FIG.7.14 5S INSOLATION AND WIND SPEED 146

FIG.7.15 PCC BUS VOLTAGES WITH SWAPSC UNDER INSOLATION AND WIND SPEED CHANGE 146

FIG.7.16 SMOOTHING OF PV AND WIND FARM POWER WITH BESS UNDER INSOLATION AND WIND SPEED CHANGE

147

FIG.7.17 BESS PERFORMANCE WITH SOC CONTROL UNDER INSOLATION AND WIND SPEED CHANGE 


\section{List of Tables}

TABLE 2.1 PV CAUSED POWER QUALITY ISSUES

TABLE 5.1 CONTROL STRATEGY MAP FOR OPERATION MODES UNDER REACTIVE POWER

TABLE 5.2 PV INVERTER SPECIFICATIONS

TABLE 6.1 CONTROL STRATEGY MAP FOR OPERATION MODES UNDER REACTIVE POWER COORDINATED CONTROL 


\section{Summary}

Renewable energies such as wind power and solar energy have become alternatives to fossil energy due to the improved energy security and sustainability. This trend leads to the rapid growth of wind and Photovoltaic (PV) farm installations worldwide. Power electronic equipments are commonly employed to interface the renewable energy generation with the grid. The intermittent nature of renewable and the large scale utilization of power electronic devices bring forth numerous challenges to system operation and design. Methods for studying and improving the operation of the interconnection of renewable energy such as wind and PV are proposed in this Ph.D. dissertation.

The modeling of a grid connected three phase PV system are presented, and equipped with a multi-objective controller including Power Quality Control (PQC) and Fault Ride Through Control (FRTC). PQC is activated to perform voltage flicker suppression, harmonic reduction and unbalance compensation. FRTC improves the dynamic performance of PV generation during system contingency by the coordination of controls of the PV power electronics interface, DC-DC booster and DC-AC inverter. Genetic algorithm is adopted to find the optimized parameters for the proposed controller. However, the control design alone of PV converters cannot solve the discontinuity of active power supply and hence in a reliable power system with high penetration of PV energy, energy storage is needed to address the active power variations. A novel supervisory control scheme is designed to coordinate PV and battery inverters to provide high quality power to the grid. This proposed control scheme provides a comprehensive solution to both active and reactive power issues caused by the intermittency of PV energy. A novel real-time experimental method for connecting physical PV panel and battery storage is proposed, and the proposed coordinated controller is tested in a Hardware in the Loop (HIL) experimental platform based on Real Time Digital Simulator (RTDS).

This work also explores the operation and controller design of a microgrid consisting of a direct drive wind generator and a battery storage system. A Model Predictive Control (MPC) strategy for the AC-DC-AC converter of wind system is derived and implemented to capture the maximum wind energy as well as provide desired reactive power. The MPC increases the accuracy of maximum wind energy capture as well as minimizes the power oscillations caused by varying wind speed. An advanced supervisory controller is presented and 
employed to coordinate the operation of wind farm and battery system in the microgrid. The control strategy ensures the power balance while regulating the PCC bus voltage within acceptable range in both grid-connected and islanded operation.

The aforementioned control algorithm works only around the designed operation point, however; due to high variability and uncertainty of renewable energies, the operation point of a power system with renewable resources is unpredictable, and the operating conditions continuously change in distribution networks. A three-layers advanced optimization and intelligent control algorithm for a microgrid with multiple renewable resources is proposed. A Dual Heuristic Programming (DHP) based system control layer is used to ensure the dynamic reliability and voltage stability of the entire microgrid as the system operation condition changes. A local layer maximizes the capability of the Photovoltaic (PV), wind power generators and battery systems, and a Model Predictive Control (MPC) based device layer increases the tracking accuracy of the converter control. The proposed control scheme namely System Wide Adaptive Predictive Supervisory Control (SWAPSC) reduces the influence of the intermittency nature of the renewable resources by smoothing the output of the PV and wind generators, maintains voltage stability by providing dynamic reactive power support to the grid, and reduces the total system loss as well as maximizes the usage of battery storages. The detailed designs of the proposed SWAPSC scheme are presented and tested on an IEEE 13 node feeder with a PV farm, a wind farm and two battery-based energy storage systems. 


\section{Chapter 1. Introduction and Objectives}

\subsection{Background of PV and Wind Generation}

Distributed generation can be used to increase operational margin and reduce net loss by providing generation to nearby load in distribution networks. A microgrid is essentially a countermeasure to implement distributed generators. Conventional energy sources such as coal and oil are increasingly losing their appeal as a viable solution to meet our future energy needs, while renewable energies such as wind power and solar energy have become alternatives to fossil energy due to the improved energy security and sustainability. This trend has led to the rapid growth of wind and PV farm installations worldwide. The European Union has set a binding target that by the year 2020,20\% of its energy supply will come from wind, PV and other renewable energy resources [1]. In the US, the Department of Energy also envisions that the wind and PV power penetration levels will exceed $20 \%$ by 2030 according to the current momentum of development in renewable resources [2]. Power electronic equipments are commonly employed to interface the renewable energy generation with the grid. For instance, a direct drive wind generator is connected to the grid via a four quarter bidirectional AC-DC-AC inverter, and a PV system is connected to the grid via DC-AC inverter [3]. The usage of these power electronic converters enables the development of advanced intelligent control algorithms to improve the performance of wind farms, PV farms and associated electric power networks.

\subsection{Challenge of Large Scale PV Generation}

PV is favorable in distributed applications due to its cleanness and inexhaustible nature. Intense concern about the adverse effects of fossil fuels on the environment and the energy crisis [4] has led to a rapid installation of the PV farms all over the world even before the potentials and challenges of PV generation have been fully understood. The interest in solar energy has reached an unprecedented level. The intermittent nature of solar power, and the large scale utilization of power electronic devices in PV generation, results in the following characteristics:

- Changing solar irradiance profile.

- Remote location of PV farm due to solar energy. 
- Harmonics.

- Lack of reactive power support.

- Inability to meet expected energy output levels without storage or auxiliary device.

These challenges result in variations in power flow, deteriorations in power quality and system stability, which are worsened by the increasing penetration levels of PV generation. A comprehensive study is required before interconnecting PV systems. Among these issues, power quality is more about dynamic characteristics and is driven by the customers and which can affect the performance of PV farm or even damage the installed power electronic devices. Analysis and countermeasures are needed to address PV related power quality issues. However, when PV is operated alone, the power generation fluctuates due to the intermittency of solar irradiation. A mismatch between demand and supply may occur and induce stability issues, and the influence may vary depending on penetration level, fault ride through ability, and reactive power support in low voltage scenario. Besides, in remote distribution network, it is desirable that distributed generators can provide local load, but the control strategy design of PV inverter cannot solve the discontinuity of active power supply. Hence, auxiliary devices are needed to address the stability issue caused by active power discontinuity induced by insolation intermittency. Storage devices such as Batteries based Storage System (BSS) are considered to be a solution to the active power intermittency related to PV generation. BSS provides operation freedom to PV generation that allows timeshifting between generation and demand. Energy storage with inverter interface in a microgrid is very similar to any other inverter-based voltage source with the abilities of plugand-play, fast response and bi-directional power flow capabilities. Having the abilities to generate and absorb active power in transient condition means that the demand and the supply can be disparate by as far as the power capabilities of the energy storage unit allow [5]. Therefore, battery provides a solution to intermittency of renewable resources like PV and wind, to provide sustainable power. With proper designed coordination control scheme, the PV and Battery can be coordinated to provide uninterruptable power supply.

Moreover, active power is not the only cause of instability. Insufficient reactive power compensation can cause voltage stability issues, which are worsened due to the remote location of PV farm. As the key component used to manage the power exchange between battery and power grid, a battery inverter is also a voltage source full bridge converter and it can be used to provide dynamic reactive power support as PV inverter. Thus, battery can also 
provide dynamic reactive power support as well as provide instant active power supply that can be used to maintain voltage stability together with PV inverter. However, in contrast to the PV panel, battery storage has only limited charging and discharging times so it is not desirable for the battery to provide reactive power compensation in normal operation mode when PV inverter has enough reactive power capacity. Furthermore, oscillations may occur when PV and battery both work in voltage control mode. But PV inverter cannot guarantee sufficient reactive power support whenever necessary because the majority of inverter capacity is used to generate active power. The battery should compensate for reactive power when the PCC voltage violates the limit and the PV inverter is at maximum reactive power output. A supervisory control scheme is also needed to coordinate PV and battery for reactive power.

\subsection{Operation of Wind Generation in Grid-connected and Islanded Mode}

The wind turbine technology can be divided into two categories: Constant Speed Constant Frequency (CSCF) and Variable Speed Constant Frequency (VSCF) [3]. Although the Squirrel Cage Inductor Generator (SCIG), as the main member of CSCF wind turbine, has the merits of economy and simplicity, it cannot capture maximum wind energy. VSCF wind generator, on the other hand, is more attractive because of the improvement in wind energy production and the flexibility of output power regulation. VSCF wind turbine is connected to the electric power network through AC-DC-AC inverter. With properly designed controller, VSCF can generate 20 to 30 percent more energy than the CSCF, and can reduce active power oscillation and provide dynamic reactive power support. In normal operation, a linear PI controller is capable of capturing the maximum energy from varying wind speed as well as delivering desired reactive power to the grid. However, the controller's performance is compromised when the wind speed changes drastically. Nonlinear control strategies such as fuzzy logic and neural network have fast response and are capable of predicting wind generation; however, they are difficult to implement in industry standard simulation tools like PSCAD/EMTDC. It is necessary to find an alternative non-linear control strategy for wind AC-DC-AC inverter to look-ahead current wind profile that optimizes system operation in transient situation.

In addition, the distribution network with wind generation is often located in a remote area and connects with the main power grid through only one transmission line. Once a fault occurs, the transmission line has to be disconnected for protection purposes. Therefore, this configuration has low safety level and cannot ensure the supply of the electricity to the critical 
load. The traditional way of increasing the reliability of the power supply is to install another transmission line as a backup. However, the major drawback of this set up is the high cost. With proper control strategy, wind farm and battery storage can provide fast response and are able to control the output current regardless of AC system voltage. Therefore, an autonomous control strategy can be a solution to maintaining power supply when the distribution network is disconnected from the main grid. Also, a novel coordination control is needed because the control objectives in grid-connected mode and island mode may differ.

1.4. Optimal Operation of a Microgrid With High Penetration Level of Wind and PV

\section{Generations}

One of the major challenges associated with the large scale utilization of renewable energy is the intermittent nature of renewable energy, leading to unexpected fast power variations of the power system thereby pushing the operating points of the power system closer to their stability limits. Moreover, due to high variability and uncertainty of renewable energies, the operation point of a power system with renewable resources is unpredictable, and the operating conditions continuously change in distribution networks. The integrated renewable resources should be able to adjust their outputs to track the changes in the operation conditions of the power system, indicating the dynamic regulation of renewable resources, and hence the optimal operation of the microgrid.

Robust nonlinear control theories feature fast response and are independent of the operation point. The main limitation of these nonlinear controllers is that they can only improve the system stability in certain area and little system-wide optimization is achieved since only local power system quantities are monitored. A Wide Area Control (WAC) is necessary for a microgrid since it can find the optimal operation point of the entire power system and hence mitigate the voltage instability as well as improve grid dynamic performances.

\subsection{Objectives of Research}

In summation, this work makes the following contributions to the dynamic analysis of distribution networks with renewable resources integration:

- The detail modeling of grid connected PV systems, wind systems and BSSs in PSCAD and RTDS. 
- The design of control strategy to mitigate power quality issues related to PV integration including.

- The implementation of genetic algorithm in finding the optimal PI parameters used in PV inverters.

- The design of coordinate controller to supervise the active and reactive power output of PV and battery based on power system quantities.

- The novel design of a real-time hardware in the loop experimental platform based on Real Time Digital Simulator (RTDS) for testing the operation and control design of a microgrid with physical PV panel and battery storage.

- The implementation of MPC strategy to capture the maximum wind energy as well as provide desired reactive power.

- The implementation of island control to a wind-Battery hybrid system that enables the sustainable power supply even when the hybrid system is isolated from the power grid.

- The design of System Wide Adaptive Predictive Supervisory Control (SWAPSC) for a microgrid with multi renewable energy integration, aims to reduce the influence of the intermittency nature of the renewable resources by smoothing the output of the PV and wind generators, maintains voltage stability by providing dynamic reactive power support to the grid, and reduces the total system loss as well as maximize the usage of battery storages. 


\section{Chapter 2. Literature Review}

In this chapter, a brief introduction is given of the previous work done by other researchers, including: (1) the causes of power quality related to PV integration and the various mitigation methods, (2) the existing modeling and supervisory control of microgrid with different renewable resources, (3) the optimization method using the genetic algorithm, (4) different island control techniques designed for autonomous operation when the microgrid is isolated from the main grid, (5) nonlinear adaptive control method for controlling the real and reactive power of renewable energy, and (6) the system wide control method for optimum operation of the microgrid in system level.

\subsection{Power Quality Control}

Some general classes of power quality issues related to three phase PV system are as listed below [6].

- Voltage flicker

- Harmonics

- Transient voltages

- Voltage unbalance

This section focuses on the mitigation of voltage flicker, harmonics, transient voltages and voltage unbalance. The causes and the existing mitigation methods of these power quality issues are analyzed in the following section.

\subsubsection{Voltage flicker}

Voltage flicker is the frequent voltage change ranging from $0.5 \mathrm{~Hz}$ to $35 \mathrm{~Hz}$ when subject to changes in the illumination intensity [7]. Voltage flicker is caused by rapid voltage changes which are normally generated by large time-varying loads like electric arc furnaces. Domestic lighting, power electronic devices and other devices like computers can be affected by voltage flicker. The frequent switching of PV inverters, due to rapid change of PV active and reactive power output can induce flickers. In addition, poorly designed control strategies with respect 
to maximum power point tracking (MPPT), inverter control and the triggering algorithm aggravate the flicker issue.

Reference [7] presents a method to measure flicker by using $P_{s t}$ (short term flicker index) meter. According to the authors, the most observable flicker frequency is from $5 \mathrm{~Hz}-15 \mathrm{~Hz}$. References [8] and [9] present control strategies for STATCOM to compensate voltage flicker. Voltage flicker can be suppressed by maintaining voltage at the PCC within certain range. The traditional control strategy of STATCOM for voltage control may cause a reverse effect, since the voltage flicker may not always be in phase with the fundamental voltage. In such a scenario, the operation of STATCOM equipped with fundamental compensation will increase the magnitude of flicker.

\subsubsection{Harmonics}

Harmonics in a PV system are attributed to two causes [10].

- Causes internal to PV: Dead time, discrete time control, calculation time of the current controller.

- Causes external to PV: Voltage distortion in a power system (existing resonance points), interconnection strategy and radiation level

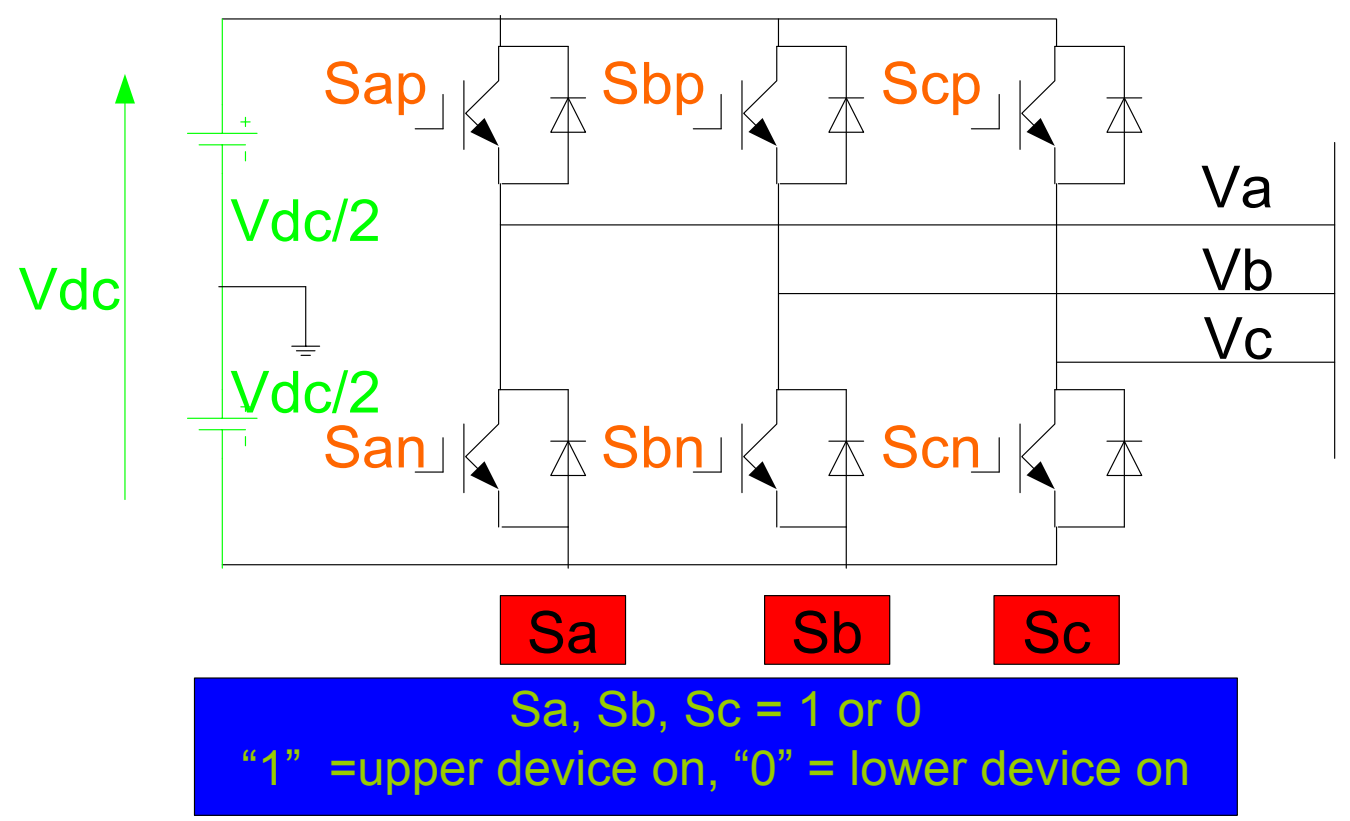

Fig.2.1 A voltage source inverter 
The internal causes are primarily related to the type of power electronic devices in a PV farm. One of the power electronic devices is the DC-AC inverter of a grid connected PV system that determines the harmonics. Fig.2.1 shows a typical inverter for a PV system.

For a three phase voltage inverter as shown in Fig.2.1, the phase voltage can be written as,

$$
\begin{gathered}
V_{a}=\left(S_{a}-\frac{1}{2}\right) \cdot V_{d c}=\left(2 S_{a}-1\right) \cdot \frac{V_{d c}}{2} \\
\frac{V_{a}}{\frac{V_{d c}}{2}}=\left(2 S_{a}-1\right)=\alpha \sin \left(\omega_{0} t+\varphi\right)+\sum_{n=1}^{\infty} \frac{4}{k \pi} \sin \left\{\frac{n \pi}{2}\left[\alpha \sin \left(\omega_{0} t+\varphi\right)-1\right]\right\} \cos \left(n \omega_{s} t\right)
\end{gathered}
$$

Using Double Fourier Analysis, the amplitude of fundamental voltage is $\alpha \frac{V_{d c}}{2}$, amplitude of $n \omega_{s} \pm k \omega_{0}$ harmonic components is $\frac{4 V_{d c}}{n \pi} J_{k}\left(\frac{\alpha n \pi}{2}\right)$, where $k=0,2,4, \ldots$ for $n=1,3,5 \ldots$ and $k=1,3,5 \ldots$ for $n=2,4,6 \ldots[10] . J_{k}$ is the $k$-th order Bessel function, $\omega_{0}$ is the system fundamental frequency, $\omega_{s}$ is the switching frequency, and $\alpha$ is the modulation index.

It can be seen from equations (2.1) and (2.2) that the harmonic components of a PV inverter have the following characteristics,

- The fundamental component has large variation in magnitude determined by the modulation index which in turn depends on the solar radiation and control strategy.

- None of the harmonics are dominant.

However, field tests in references $[11,12,13,14,15]$ show that the observed dominant harmonics associated with grid connected PV plants are $3^{\text {rd }}, 5^{\text {th }}, 7^{\text {th }}, 11^{\text {th }}$ and $13^{\text {th }}$ orders. These field test results along with equation (2.2) prove that causes external to PV have a great impact on dominant harmonics. Total Harmonic Distortion (THD) is used to quantify the harmonic pollution and is given by equation (2.3).

$$
T H D=\frac{\sqrt{I_{2}^{2}+I_{3}^{2}+I_{4}^{2}+\cdots+I_{n}^{2}}}{I_{1}}
$$

where, $I_{1}$ is the fundamental current and $I_{n}$ is the $n$-th order harmonic current (for $n=2,3,4 \cdots)$. Reference [16] shows comparison of THD in current waveform with prior-voltage distortion and without prior-voltage distortion. Prior-voltage distortion results in higher THD with harmonic orders of $3^{r d}, 5^{\text {th }}$ and $7^{\text {th }}$ matching the frequencies of voltage distortion 
making them dominant. The harmonic current during the day is proportional to the fundamental current. However, during sunset and sunrise, the harmonic current proportions significantly increase and according to equation (2.3), the THD is much higher $[17,18]$. Apart from voltage distortion and irradiation level, harmonics can be caused by interconnection schemes. Traditionally transformers isolate the inverters from the grid, however, it has been noticed that they result in higher THD [18].

Generally, for small scale (up to $10 \mathrm{KW}$ ) and medium scale (10 to $1000 \mathrm{KW}$ ) PV systems, the harmonic mitigation is not essential, however for large scale (1 to $10 \mathrm{MW}$ ) PV with high THD, harmonic mitigation is essential. Several passive and active methods have been presented in literature and applied in industries. The passive reduction employs a first order LC filter for economy and simplicity. However, when prior-voltage distortion changes, the THD varies and makes it difficult to calculate the capacity of each LC filter branch and configuration. STATCOM employs an active mitigation method due to its fast response and can generate any voltage with desired frequency (lower than switch frequency) and magnitude (lower than rated DC voltage value). Reference [19] utilizes a STATCOM harmonic current reduction control that extracts specific harmonic voltage using Fourier Coefficient Expansions (FCEs). However, it is difficult to extract specific harmonic voltage since the harmonic components have lower order harmonics and high frequency ripple as a result of high switching frequency.

\subsubsection{Transient voltage}

Transient voltages are caused by sudden changes in load or supply resulting in active or reactive power unbalances apart from short circuit faults causing line tripping [20,21]. Variable PV active power due to intermittencies in solar radiation can cause transient voltage. When the active power is higher than local demand, there is a reverse power flow on the grid which in turn causes voltage rise even with small scale PV due to remote location. When the active power is lower than local demand, the power is drawn from the grid which results in a voltage drop and an increase in line losses. The variable PV active power creates voltage rises and drops which are more pronounced in a weak system where voltage is sensitive to an active power change.

Active power can be balanced by battery storage where battery is charged when system voltage is higher than the upper set value, and discharged when system voltage is lower than 
the lower set value. Reference [22] utilizes a battery charging scheme and introducing dead band for charging and discharging to protect the battery storage from excessive charging frequency and current variation.

Considering the system $\mathrm{R} / \mathrm{X}$ ratio is normally low, voltage change is also highly related to reactive power. Lack of dynamic reactive power support leads to voltage issues such as low voltage or over voltage and general ways to mitigate these issues in a distribution system are:

- Changing the secondary transformer voltage by adjusting the tap position [23].

- Switching on or off capacitor compensators.

- Installing transformer/voltage regulators [24].

- Installing dynamic reactive power compensators, such as SVC and STATCOM.

The traditional ways to provide reactive power compensation have obvious limitations. Changing secondary voltage of the transformer doesn't change the reactive power of the system. Capacitors and regulators cannot perform linear compensation and the switching operation creates disturbance in the system. What is more, the mechanical switches used in regulators can only be operated for a limited time and their massive use should be limited due to the wear and tear. In addition, the operation of mechanical switches is slow (from a few seconds to a few minutes) and hence cannot respond to rapid voltage oscillations.

[25] and [26] present several reactive-power control schemes to optimize the dynamic performance of a PV generation. However, traditional reactive power control doesn't incorporate active power control during system contingency, which results in overcharging of the coupling capacitor bank and overshoot of active power output after a fault clearance. Most of the control strategies presented in the literature for regulating voltage fluctuations use output current as a reference variable and assume the terminal voltage as constant. If the terminal voltage is lower than the rated value, the close loop control increases the reference value of output current to meet the power requirements, which can further damage the PV inverter.

SVC and STATCOM can regulate voltage fluctuations due to their capability of providing fast and linear reactive power compensation. Over-reactive power compensation can also result in active power balance. SVC and STATCOM can also raise the fault ride through ability of PV farm and can hold the terminal voltage of PV plant within acceptable levels. Due to a faster 
response and less dependence on terminal voltage value, STATCOM has better performance than SVC.

\subsubsection{Voltage unbalance}

Unbalanced load, unsymmetrical system fault or un-transposed lines can cause voltage unbalance. Among these causes, unbalanced load is the prominent cause in a distribution network. With traditional control strategies, devices with Voltage Source Inverter (VSI) interface, such as PV farm and BSS, can be greatly affected by the unbalance voltage. In severe cases, voltage unbalance can cause the disconnection of PV farm for over current protection $[27,28]$. By compensating for unbalanced load at a load terminal the system voltage and current balance can be reduced. An unbalanced signal can be decomposed to positive, negative and zero sequences by using symmetrical component decomposition method. The negative sequence component is the primary unbalance source in a three phase three wire system as the zero sequence component doesn't exist. As shown in Fig.2.2 for the unbalance control scheme an output current similar in magnitude but reverse in direction of the negative sequence component is injected.

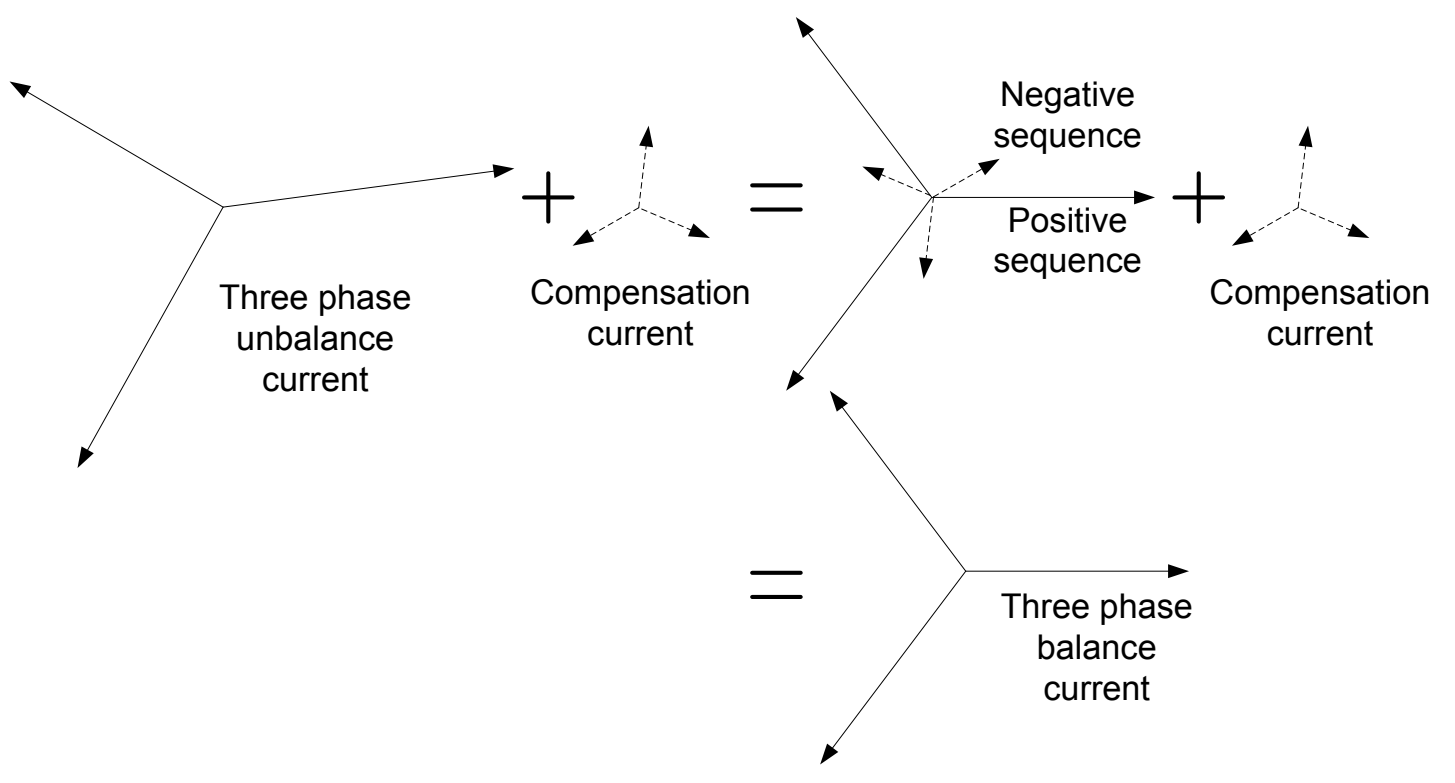

Fig.2.2 Unbalance Compensation

Unbalance compensation control using STATCOM is discussed in [29,30,31]. A novel controller which can deal with voltage unbalance caused by utility is presented in [29]. The author analyzes the compensation characteristics and the optimization of operation point of 
STATCOM controller for unbalance compensation and compares the compensation loss of Voltage Controlled Voltage Source (VCVS) with Voltage Controlled Current Source type STATCOM. A new method to detect the negative sequence component of Point of Common Coupling (PCC) buss voltage during an unbalance fault is presented in [30]. The author proposes two different kinds of voltage unbalance control methods, direct voltage regulation and decoupled voltage regulation. The first method shows a fast transient response in steady state, and the second one has inner current protection and can deal with any voltage unbalance caused by utility or load. The source [31] presents a three phase independent control to address voltage unbalance caused by an electric arc furnace. The three phase voltages are decoupled and compensated separately. Table 2.1 gives an overall description of power quality issues of PV.

TABLE 2.1

PV CAUSED POWER QUALITY ISSUES

\begin{tabular}{|c|c|c|c|}
\hline $\begin{array}{c}\text { Power quality } \\
\text { issues }\end{array}$ & Cause & Hazard & Mitigation \\
\hline Voltage Flicker & Rapid change of PV output power & Health issues. & STATCOM \\
\hline Harmonics & $\begin{array}{l}\text { Voltage distortion in power system; } \\
\text { Interconnect strategy; Insulation level; } \\
\text { Dead time, discrete time control, } \\
\text { calculation time of the current } \\
\text { controller. }\end{array}$ & $\begin{array}{l}\text { Degradation of supply } \\
\text { quality; } \\
\text { Reduction of equipment } \\
\text { reliability; } \\
\text { Shortening of component } \\
\text { life expectancy. }\end{array}$ & $\begin{array}{l}\text { LC Filter } \\
\text { STATCOM }\end{array}$ \\
\hline $\begin{array}{l}\text { Transient } \\
\text { voltages }\end{array}$ & $\begin{array}{l}\text { Reactive power unbalance; } \\
\text { Active power unbalance. }\end{array}$ & $\begin{array}{c}\text { Unexpected } \\
\text { disconnection of PV } \\
\text { plant; } \\
\text { Damage to generators }\end{array}$ & $\begin{array}{c}\text { SVC } \\
\text { STATCOM }\end{array}$ \\
\hline $\begin{array}{l}\text { Voltage } \\
\text { unbalance }\end{array}$ & $\begin{array}{l}\text { Unbalanced load; } \\
\text { Unsymmetrical system fault; } \\
\text { Un-transposed lines. }\end{array}$ & $\begin{array}{c}\text { Increase of losses; } \\
\text { Reduction of reliability; } \\
\text { Shutdown of load with } \\
\text { high sensitivity }\end{array}$ & STATCOM \\
\hline
\end{tabular}

\subsubsection{Section Summary}

It is seen from table 2.1 that, the most effective way to mitigate power quality issues related to PV integration is using STATCOM. However, one of the major drawbacks of STATCOM is its high cost. As the key component used to manage the power exchange between PV and power grid, a PV inverter is also a voltage source full bridge converter which is similar to a STATCOM. In this work, power quality control for PV inverters has been designed to perform STATCOM applications as shown in Chapter 4. With the proposed control, voltage flicker, transient voltage, and voltage unbalance can be compensated by PV inverter. Moreover, this 
controller can eliminate up to the $100^{\text {th }}$ order harmonic under the premise of sufficient capacity.

\subsection{Microgrid Modeling and Coordination Controller Design}

\subsubsection{PV panel modeling}

Photovoltaic effect forms the basis of PV cell modeling where solar energy is converted to electricity under PV panel insolation. A PV generator is a combination of a large number of PV cells connected in series and in parallel. It can be expressed as a current source with an anti-parallel diode as shown in Fig.2.3 [32,33,34,35,36],

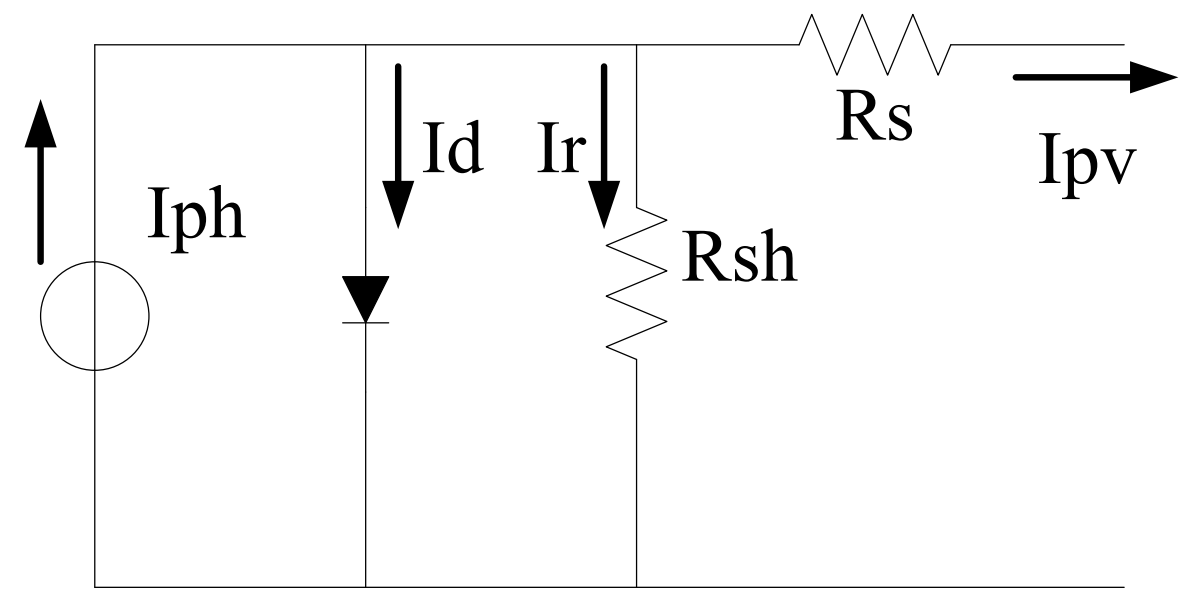

Fig.2.3 An equivalent PV cell circuit

where $I_{p h}$ is the current directly generated by the insolation, $I_{d}$ is the current flow through the diode, $R_{s h}$ is the shunt resistor of PV module, $I_{r}$ is the current flow through $R_{s h}, R_{S}$ is the series resistor of the PV panel, and $I_{p v}$ is the output current of PV module.

$I_{p v}$ can be calculated from (2.4), [32]

$$
I_{p v}=I_{p h}-I_{d}-I_{r}
$$

$I_{d}$ and $I_{p h}$ are given by (2.5) and (2.6), [34],

$$
\begin{gathered}
I_{p h}=\left[I_{s c}+K_{i}\left(T-T_{n}\right)\right] \frac{G}{G_{n}} \\
I_{d}=I_{0}\left[e^{\frac{q\left(V_{p v}+I_{p v} R_{s}\right)}{\alpha K T}}-1\right]
\end{gathered}
$$


$I_{S C}$ is the short circuit current of the PV module, $K_{i}$ is the short circuit current/temperature coefficient, $G$ is the solar insolation in $W / \mathrm{m}^{2}, G_{n}$ is the nominal solar insolation, $V_{p v}$ is the PV module output voltage, $q$ is the electron charge $\left(1.6 \times 10^{-19} \mathrm{C}\right), K$ is the Boltzmann constant $\left(1.38 \times 10^{-23} \mathrm{~J} / \mathrm{K}\right), \alpha$ is the ideality factor and varies between 1.0 and 1.5 (1.2 is chosen), $T$ is the temperature in Kelvin of the p-n junction, and $I_{0}$ is the diode saturation current expressed in (2.7) [34],

$$
I_{0}=I_{0, n}\left[e^{\frac{q E_{g}}{\alpha K}\left(\frac{1}{T_{n}}-\frac{1}{T}\right)}-1\right]
$$

$E_{g}$ is the band gap energy of the diode, $T_{n}$ is the nominal temperature, and $I_{0, n}$ is the nominal saturation current which can be calculated by (2.8), [34]

$$
I_{0, n}=\frac{I_{s c}}{e^{\frac{V_{o c}}{\alpha V_{t}}}-1}
$$

$V_{o c}$ is the open circuit voltage of the PV module, $V_{t}$ is the thermal voltage of the PV cell, which is defined as $V_{t}=N_{s} K T / q$, and $N_{s}$ is the number of PV cells in a series.

Based on (2.4) - (2.8), a PV generator model is developed in EMTDC/ PSCAD as shown in Fig.2.4.
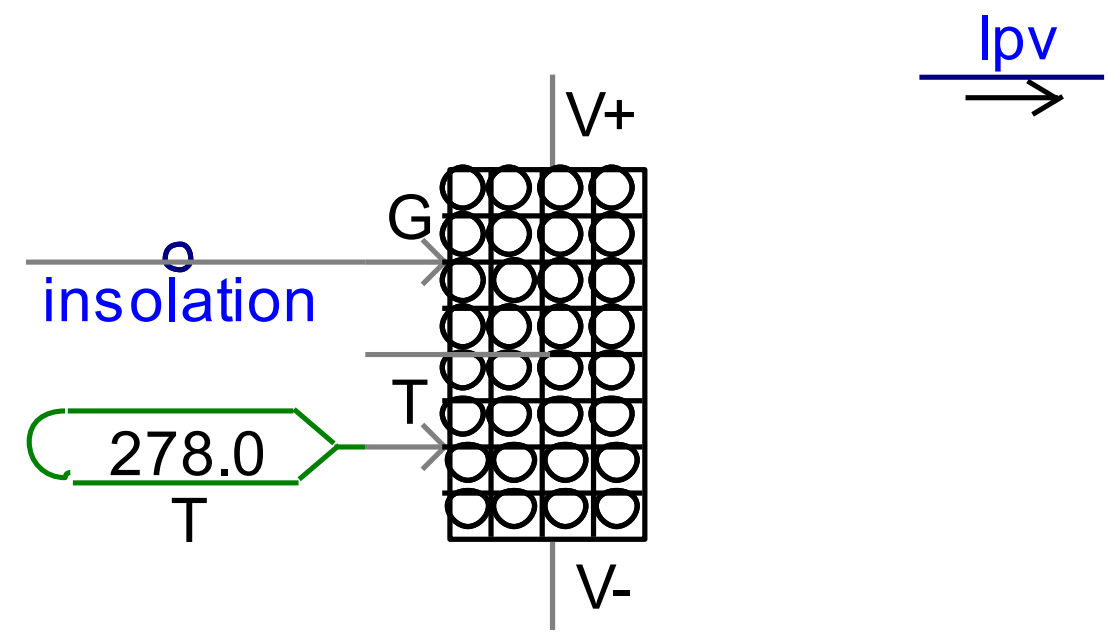

Fig.2.4 PV generator model in PSCAD

The PV generator model functions as a controllable DC current source and the value of the current depends on insolation $G$, temperature and the PV panel terminal voltage. 


\subsubsection{PV power electronic interface modeling}

The power electronic interface of PV consists of a PV DC-DC booster and a DC-AC inverter. The DC-DC booster is used to regulate the terminal voltage of the PV panel and boost the voltage for AC system integration. Reference [34] studies the V-I and P-V characteristics of a PV panel. It is shown that there is a single maximum power point of the PV generator for a specified operating condition, and it is desired that the PV generator operates at this point. The author proposes a hill top algorithm which adjusts the PV terminal voltage and obtains the maximum power output. Reference [37] presents a new method to get PV instantaneous V-I characteristics and then uses this for MPPT control. The V-I characteristic curve is obtained by PV short and open circuit while calculating terminal voltage and current. Reference [38] introduces a variable time step MPPT which can increase the speed of maximum power tracking without causing oscillations. The method can automatically adjust the calculation step based on the output power. However, for PSCAD application, these methods are very difficult to implement. Since this work focuses on the system level control strategy design, a modified Perturbation and Observation (P\&O) method is adopted due to its accuracy and simplicity.

The DC-AC inverter of a grid connected PV system is used to balance the average power delivery from the PV generator to PCC as well as provide desired reactive power to the power system. $d q 0$ transformation is used in this work to decouple the control of active and reactive power. Different books use different ways to define $d$ and $q$ axis, and correct modeling can be achieved either way. Here, the $d q 0$ transformation described in [39] is used and $q$-axis is defined as 90 degree ahead of $d$-axis. Equations (2.9) and (2.10) shown the transformation matrix used in this section.

$$
\begin{aligned}
& {\left[\begin{array}{l}
q \\
d \\
0
\end{array}\right]=\frac{2}{3} \cdot\left[\begin{array}{ccc}
\cos (\theta) & \cos \left(\theta-\frac{2 \pi}{3}\right) & \cos \left(\theta+\frac{2 \pi}{3}\right) \\
\sin (\theta) & \sin \left(\theta-\frac{2 \pi}{3}\right) & \sin \left(\theta+\frac{2 \pi}{3}\right) \\
\frac{1}{2} & \frac{1}{2} & \frac{1}{2}
\end{array}\right] \cdot\left[\begin{array}{l}
a \\
b \\
c
\end{array}\right]} \\
& {\left[\begin{array}{l}
\mathrm{a} \\
\mathrm{b} \\
\mathrm{c}
\end{array}\right]=\left[\begin{array}{ccc}
\cos (\theta) & \sin (\theta) & 1 \\
\cos \left(\theta-\frac{2 \pi}{3}\right) & \sin \left(\theta-\frac{2 \pi}{3}\right) & 1 \\
\cos \left(\theta+\frac{2 \pi}{3}\right) & \sin \left(\theta+\frac{2 \pi}{3}\right) & 1
\end{array}\right] \cdot\left[\begin{array}{l}
\mathrm{q} \\
\mathrm{d} \\
0
\end{array}\right]}
\end{aligned}
$$


where, $\theta=\int_{0}^{t} \omega d t$, and $\omega$ is the angular velocity of $d q 0$ reference frame.

\subsubsection{Battery cell modeling}

The storage system has a heavy influence on the integration of not just PV but all renewable energy. Therefore, it is very important to develop an accurate battery set which can be conveniently used in simulation study. There have been several different kinds of battery models reported in the literature and these models can be divided into three categories: experimental, electrochemical and electric circuit based [40,41,42,43]. However, the modeling of the experimental and electrochemical type battery models is inaccurate due to the State of Charge (SOC) estimation. In this work, an electric circuit based battery model is chosen, and it includes a controllable voltage source in series with an internal resistance to represent the battery packs. The magnitude of the voltage source is determined by the battery current, initial status, and battery operation mode. Fig.2.5 shows the battery model with the charging and discharging dynamics.

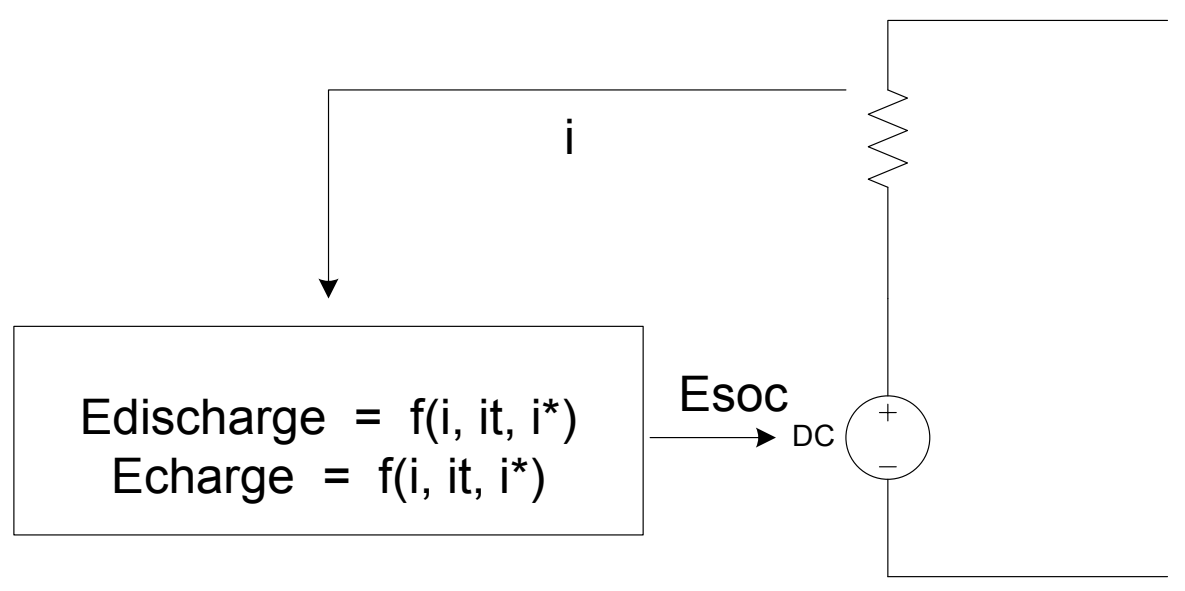

Fig.2.5 Equivalent circuit model of a battery cell

where, the discharging Voltage $E_{\text {discharge }}$ can be calculated from (2.11) [41],

$$
E_{\text {discharge }}=E_{0}-K \cdot \frac{Q}{Q-i t} \cdot i^{*}-K \cdot \frac{Q}{Q-i t} \cdot i t+A \cdot e^{-B \cdot i t}
$$

The charging voltage $E_{\text {charge }}$ is given by (2.12) [41],

$$
E_{\text {charge }}=E_{0}-K \cdot \frac{Q}{|i t|+0.1 \cdot Q} \cdot i^{*}-K \cdot \frac{Q}{Q-i t} \cdot i t+A \cdot e^{-B \cdot i t}
$$

State Of Charge (SOC) is given by (2.13) [40], 


$$
\text { SOC }=\frac{Q-i t}{Q} \times 100 \%
$$

where $E_{0}$ is the constant voltage, $Q$ is the maximum battery capacity, it is the extracted capacity, $i^{*}$ is the low frequency current dynamics, $K$ is the polarization constant or polarization resistance, $A$ is the exponential voltage and $B$ is the exponential capacity. Among these variables, $E_{0}, Q, K, A$ and $B$ are fixed and only depend on the manufacturer's data. $i^{*}$ and it are determined from the current flowing through the battery.

\subsubsection{Battery power electronic interface modeling}

The typical battery power electronic interface consists of a DC-DC buck booster and a DC-AC inverter [44]. The desired function for a DC-DC buck booster is to maintain the voltage of the DC coupling capacitor constant by charging or discharging the battery. The buck-booster circuit used in this work is shown in Fig.2.6,

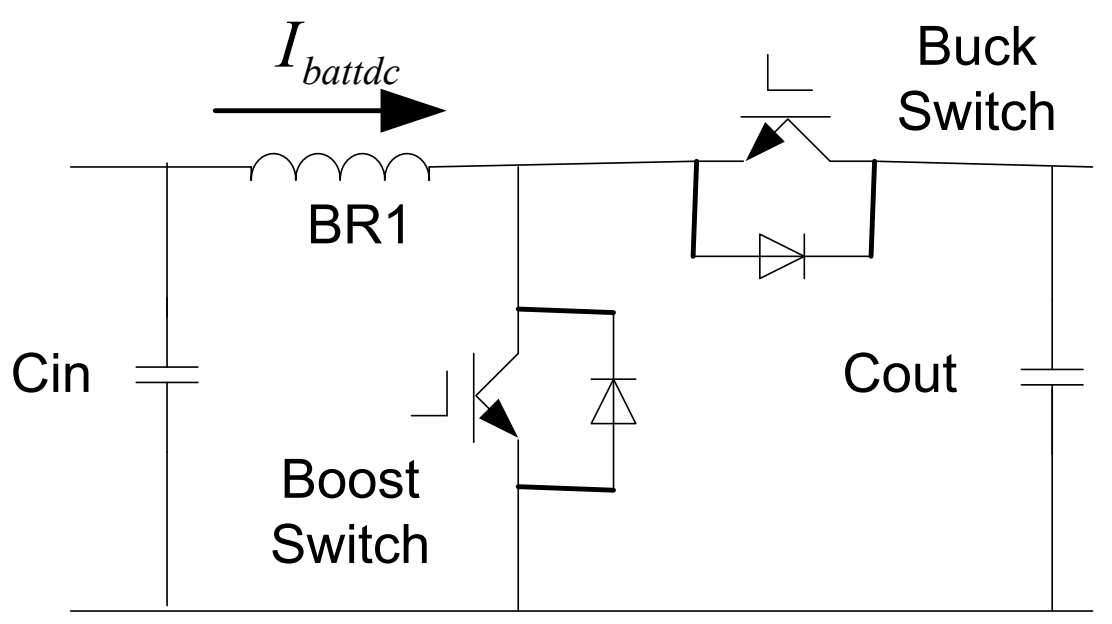

Fig.2.6 Buck- Boost circuit of BSS

Basically, battery storage has two operation modes: charging and discharging. The buck and booster switches shown in Fig.2.6 are used for charging and discharging the battery respectively. The operation modes of the battery are selected through changing the status of booster and buck switches, and only one switch can be activated at a time. Normally, one voltage loop is sufficient to control the buck switch due to the simplicity of the buck circuit. However, one more outer current loop is necessary to the booster controller because the discharging of the battery is realized by the energizing and de-energizing inductor BR1 and thus $I_{b a t t d c}$ needs to be controlled. 
The desired functions for battery DC-AC inverter are to:

- Generate sufficient active power when the supply is lower than demand.

- Absorb surplus active power when the supply is higher than demand.

However, due to its VSI based structure, BSS can also provide dynamic reactive power supply to the power grid when necessary. This study explores the ability of BSS thoroughly and proposes a new operation mode (idle mode) to avoid frequent operational changes of BSS.

\subsubsection{Microgrid configuration and control}

Microgrid is essentially a countermeasure to implementing distributed generators. A modern microgrid is normally based on small scale renewable resources such as wind, PV, diesel, BSS, and electric vehicle due to the rapid development of renewable technologies. Prior works detailing modeling and designs of hybrid systems with different renewable resources were presented in $[45,46,47,48,49]$. A microgrid can be DC or AC or a combination of DC and AC network [50]. Since most of the distribution networks are AC systems, here AC microgrid is the main focus. There are a wide variety of configurations for an AC microgrid. A windbattery hybrid system is presented in [51]; the author investigates the feature of a Sodium sulfur (NAS) battery system and the reaction between battery storage and wind system during varying wind speed and induction motor start/stop. [52] presents a Fuel Cell/ Battery hybrid power system and investigates an integrated control system to regulate the output power from each energy source under different scenarios through active current sharing and source management. A wind-PV-Battery hybrid system is presented in [53], and the modeling of a PV panel, wind turbine and battery storage are described in detail. PI controller is used to regulate the active power of PV DC-DC inverter and wind AC-DC-AC converter. The simulation result shows the complementary nature of PV and wind. A PV-diesel hybrid system is present in [54]. The modeling and controller design of a diesel generator is provided and the diesel generator is used to smooth the active power output of PV farm. However, these papers investigate the modeling and individual controller design rather than coordination between different renewable resources. A microgrid's supervisory control can regulate the active power dispatch among different renewable resources as presented in $[55,56,57,58]$. References [59] and [60] present the coordination control to charge and discharge the battery based on the active power unbalance between PV and battery. Deadband is introduced to avoid unnecessary operation of battery storage. A supervisory 
control is proposed in [61] to maintain the system frequency by adjusting the PV and diesel output powers. However, reactive power coordination between battery and PV has received little research attention. Insufficient reactive power compensation can cause voltage stability issues which are worsened by the remote location of renewable resources.

\subsubsection{Section summary}

The existing work on microgrid modeling and control either focus on modeling and individual controller design rather than coordination between different renewable resources or pay little attention on the coordination of reactive power. A comprehensive coordinated control algorithm is thus proposed in Chapters 5 and 6 to smooth the transient active and reactive power delivery of the PV and wind power system utilizing a battery-based energy storage unit and the grid.

\subsection{Island Control}

In grid connected mode, the synchronous generators in main power grid maintain the voltage and frequency of the microgrid constant even when there is no power exchange on the interconnection transmission line. All the synchronous signals used in controlling renewable devices, such as PV inverter and wind converter, can be obtained directly from measurements. In a standalone situation, the network is isolated from the main AC supply system. There are no synchronous alternators that can provide voltage and frequency references [62]. Those references are crucial in the control of VSI which is the most commonly used interface between the distribution generator and load. In an islanded system with multiple VSIs operating in parallel, at least one of the VSIs should be selected to control the voltage and frequency. Reference [63] uses a diesel generator as the main source during island mode. The diesel inverter changes from current control to voltage control when the microgrid is disconnected from the power grid. However, the diesel generator can be treated as an infinite source due to the relatively stable and continuous energy supply. PV generation, on the other hand, is determined by the insolation intensity which is intermittent. Reference [64] proposes an island controller which uses battery storage as the main source. The controller adjusts the output of the battery to provide a constant voltage amplitude and frequency at PCC. However, in a location with frequent change of weather and load, the life span of battery is compromised due to the frequent operational changes between charging and discharging. A decentralized voltage droop controller is used in [65] to share the power 
generation among wind turbines based on the available wind power during island mode. With this controller, all the wind turbines work together to supply the entire power and the maximum wind energy is utilized. However, this controller requires a control change for all the interconnected wind turbines which can increase the expense and complexity of the operation. A standalone operation of a direct drive wind turbine and doubly fed induction wind turbine with maximum power extraction capability is proposed in [66]. BSS and dump load are used to assist the wind turbine to provide constant voltage and frequency. A simple coordination control between battery and wind is also presented to manage the active power flow between supply and demand. Dump load absorbs excessive power under overgeneration. However, the battery and wind are connected to the same DC bus which increases the fault possibility and reduces the operation flexibility. Also, dump load is expensive and can cause huge power losses. The transient control when the system is changing from gridconnected to island, is presented in [67]. All renewable resources are connected to the same $\mathrm{AC}$ bus. The transient system voltage before island is sampled and used as the reference angle for island control for seamless transition. However, angle shift may occur when the system is disconnected from main grid, and same voltage angle cannot be used as the reference for all renewable resources if they were not connected on the same bus. Also, it is difficult to obtain the voltage angle when the renewable resource is far from PCC and hence commutation is inevitable. Other works related to island control are presented in $[68,69,70,71,72,73]$. The control strategies of wind turbines in island mode are examined thoroughly. Only a few transaction papers investigate the standalone operation of a microgrid with renewable resources interconnected at different locations. Moreover, since the existing research shows that battery storage is necessary when the microgrid is working under islanded mode, coordinations between battery and renewable resources in islanded operation are needed to be addressed. A comprehensive coordination method proposed in Chapter 6 ensures the maximum power extraction capability of a wind turbine while regulating the PCC bus voltage within acceptable range in both grid-connected and islanded operations. A seamless transition method is also proposed to calculate the reference voltage angle for islanded operation control.

\subsection{Optimization Using Genetic Algorithm}

PI controller is the most commonly used element in converter control, and improperly selected PI parameters can influence the converter performance significantly. The optimum 
PI parameters of a control system for a single converter can be obtained without programing. However, the transfer function of a control system with multi converters working in parallel can be very complicated and not generic, relying on specific control complexity and objectives, in particular for distribution systems. Consider a parameter optimization problem where a set of variables are needed to be optimized either to maximize some goal such as profit, or to minimize the cost or some measurement of error. Genetic algorithm makes it possible to investigate a much larger range of potential solutions to a problem compared to conventional programs. Genetic algorithm doesn't require any information or knowledge about the objects, and it is currently the most prominent stochastic optimization algorithm and is widely used in power system problems [74]. The procedures of a typical genetic algorithm are shown as follow [75]:

1) Encoding and creation of the initial population of individuals.

- Encoding the individuals (or chromosomes) by determining the number and definition of all the genes in each chromosome.

- Generate the initial population of individuals by using uniform random variables.

2) Evaluation and selection.

- Choose a proper objective function (or fitness function) to evaluate the individual fitness of all the chromosomes in that population.

- Select the best-fit chromosomes to reproduce the individuals for the next generation. The greater the fitness index value, the more likely its selection for reproduction.

3) Breeding, and reproducing the next generation.

- Breed new offspring through crossover and mutation operations.

- Generate the next generation by adding the obtained offspring and replacing least-fit population with new individuals or use the most fitness individuals from the last generation.

These reproduction and selection steps are repeated until a termination condition has been reached. Common terminating conditions are [75]:

- A solution is found that satisfies the optimization criterion.

- A certain number of generations is reached.

- Allocated budget (computation time/money) is reached.

- The highest ranking solution's fitness is reaching or has reached a plateau such that successive iterations no longer produce better results. 
- Manual inspection.

- Combinations of the above.

The key idea of genetic algorithm approach is shown in Fig.2.7,

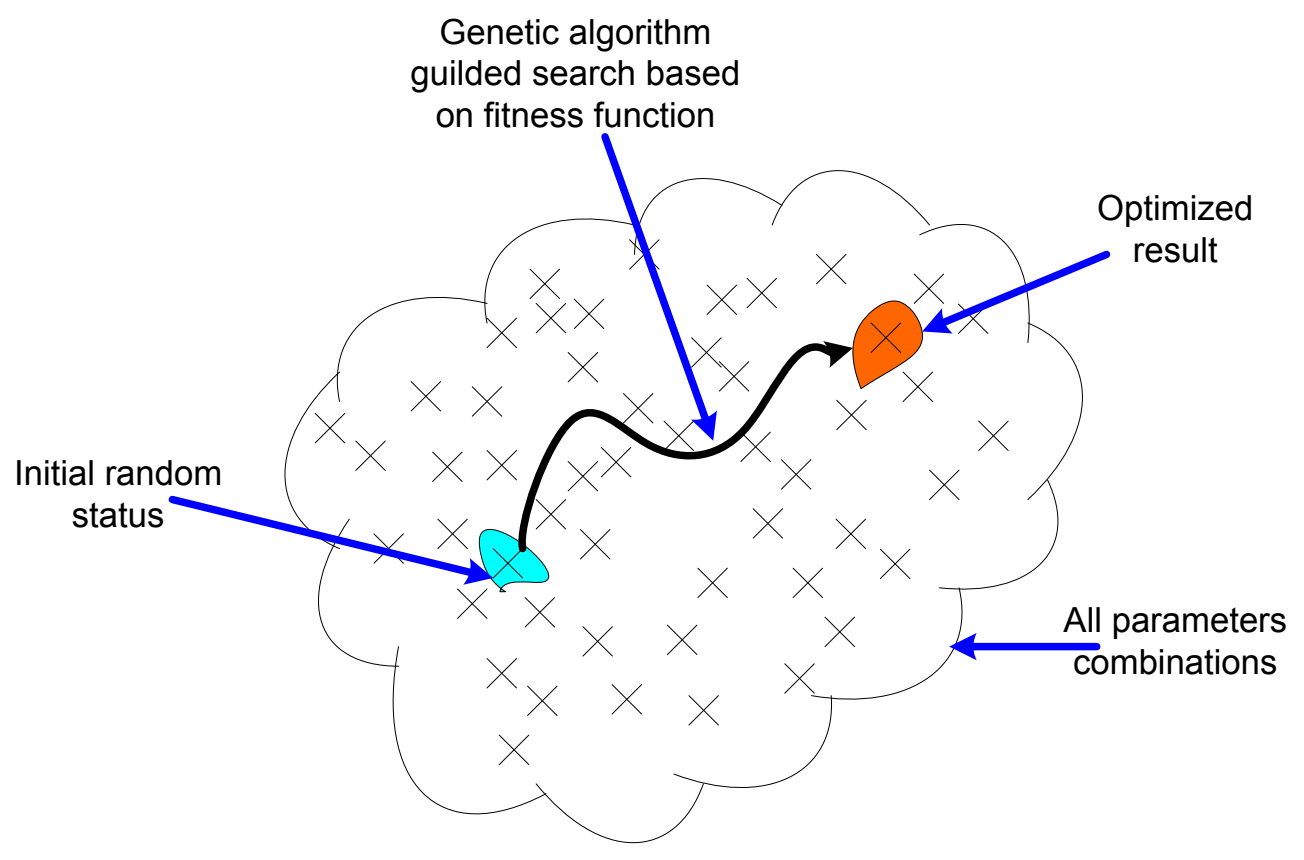

Fig.2.7 The idea of genetic algorithm

where, the initial random status is the first generation, the genetic algorithm guided search is selection and reproduction, and the optimized result is the decoded solution when termination condition has been reached. Among the three major procedures, reproduction is problem-independent and is achieved through crossover and mutation. On the other hand, the initial generation encoding and the fitness function are two main components of most genetic algorithms that are problem-dependent.

\subsubsection{Crossover}

Crossover is the process of taking two parents and producing two children, and it greatly accelerates the search in evolution of a population. The crossover operation ensures the new individuals inherit characteristics from both parents, and the ratio of characteristics from different parents is decided by the type of crossovers [76]. There are mainly two different kinds of crossover: single point crossover, and multi point crossover. Fig.2.8 shows a single point crossover: 


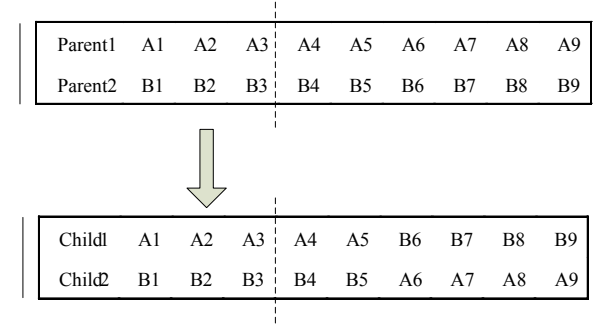

Fig.2.8 Idea of crossover

Crossover rate is a parameter to describe how often crossover will be performed. If crossover probability is $100 \%$, then all offspring are made by crossover. If it is $0 \%$, a whole new generation is made from exact copies of chromosomes from the old population.

\subsubsection{Mutation}

Mutation operation is an aleatory small change in genes and it is used to cause movement in the search space and hence restores lost information to the population [76]. Fig.2.9 shows a flipping mutation that used in chromosomes consisting of binary bits:

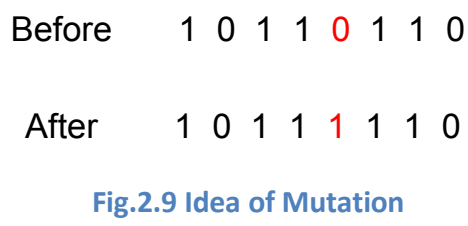

Similar mutation can also be used in chromosomes consisting of real numbers. The mutation rate decides how often parts of the chromosome will be mutated. Normally the mutation rate is much lower than crossover rate. However, it is equally essential.

\subsubsection{Population encoding}

For each and every problem, the population size is different and depends on the complexity of the optimization. A population is a collection of chromosomes (individuals), and each chromosome may describe a possible solution to a problem, without actually being the solution. A chromosome consists of a sequence of genes. Gene is the basic "instruction" for building a generic algorithm and is a digit string of arbitrary lengths.

\subsubsection{Fitness function}

For calculating the fitness, the chromosome has to be first decoded and the objective function has to be evaluated. The fitness function is problem-dependent and is used to evaluate all the 
chromosomes in one population. The fitness not only indicates how good the solution is, but also corresponds to how close the chromosome is to the optimal one [77].

\subsubsection{Section summary}

A genetic algorithm approach is adopted in chapter 5 to find the optimum PI parameters of a voltage source inverter, and the optimization result is compared with hand calculation.

\subsection{Model Predictive Control}

Model Predictive Control (MPC) is a computer control technique developed since 1970's which received increasing interest due to its flexibility and simplicity. A few applications of MPC in power system problems can be found in $[78,79,80,81]$. The application of MPC in an inverter type UPS system with an output LC filter is presented in [78]. The UPS with proposed MPC shows good performance for voltage regulation under both linear and non-linear load. [79] presents a distributed MPC strategy and aims to maintain multi-area voltage within acceptable bounds during major disturbances. The nonlinear hybrid model of the studied system is presented and all MPC units are coordinated through limited commutation. Reference [80] presents the application of MPC in induction motor drives. The authors derive the prediction model for a two-level five-phase induction motor drive, and explore all switching states according to a predefined cost function. The optimum control signals are obtained after each calculation interval. Reference [81] introduces the application of MPC in a back-to-back converter of a HVDC substation. The mathematical model of a VSI inverter is presented and a multi-objective function to increase the control accuracy as well as reduce circulation current is proposed. According to the previous research, the theory behind MPC is, in each time step, it uses the model of the system to predict the system response under finite control variables variations until a certain horizon of time. Then a cost function or objective function is applied to evaluate all system responses under different control actions and determine the optimum future control action for the next predefined time horizon. Due to similarity of the topology used in this work, the prediction model developed in $[78,81]$ is adopted here to ensure the adaptability of the wind farm converter controller. The implementation of MPC can be found in Chapter 6.

\subsection{System Wide Adaptive Control}


The classical control algorithm using PI controller works only around the designed operation point, however, due to high variability and uncertainty of renewable energies, the operation point of a power system with renewable resources is unpredictable, and the operating conditions continuously change in distribution networks. The integrated renewable resources should be able to adjust their outputs to track the changes in the operation conditions of the power system, indicating the dynamic regulation of renewable resources. Robust nonlinear control theories presented in $[82,83,84,85,86,87]$ feature fast response and are independent of the operation point. The main limitation of these nonlinear controllers is that they can only improve the system stability in a certain area and little system-wide optimization is achieved since only local power system quantities are monitored. A Wide Area Control (WAC) can find the optimal operation point of the entire power system and hence mitigate the voltage instability as well as improve grid dynamic performances $[88,89,90,91,92,93]$. The authors of $[89,90,91]$ propose different closed-loop WAC algorithm to improve the system performance by using Adaptive Critic Design (ACD). A family of ACD was proposed by Werbos $[92,93]$ as a new control technique that can reduce the computation burdens and approximate the optimal control signals by reinforcement learning and approximate dynamic programming. There are different kinds of ACD and normally it consists of three elements: a model network, a critic network, and an action network. The model network predicts the future state of the system, the critic network evaluates the control signals by approximating a cost-to-go function $J$ and the action network determines the control action at the next time step that can minimize J. Reference [92] designs a nonlinear optimal adaptive interface neuro-controller by employing a Heuristic Dynamic Program (HDP) approach for wind farm and STATCOM coordination. However, in HDP, the critic network is so trained that the cost-to-go function J is directly considered; a potential problem of being coarse is expected since the derivatives of $J$ are indirectly obtained by backpropagation through the critic network [94]. More advanced DHP is presented in [93,95]. In DHP, the critic network is so trained that the derivatives of the cost-to-go function J with respect to the output factor $Y$ is directly used rather than the cost-to-go function [[96]. [95] adopts DHP to coordinate all the generators in the system to achieve operation optimal active power flow as well as minimum fuel cost. However, detailed designs and application of ACD on multiple renewable resources have not been reported. The DHP is adopted in this work to design a System Wide Adaptive Predictive Supervisory Control (SWAPSC) scheme to smoothen the PV and wind generation output, provides dynamic reactive power support, and 
reduces the power loss as well as maximizes the usage of battery storage. The detail description can be found in Chapter 7.

\subsection{Distribution Network Modeling}

An accurate distribution network is needed to test the effectiveness of the proposed control strategy. IEEE published distribution feeders are used in this work. Take IEEE 13 bus feeder, for instance. The one line diagram of IEEE 13 bus feeder is shown in Fig. 2.10,

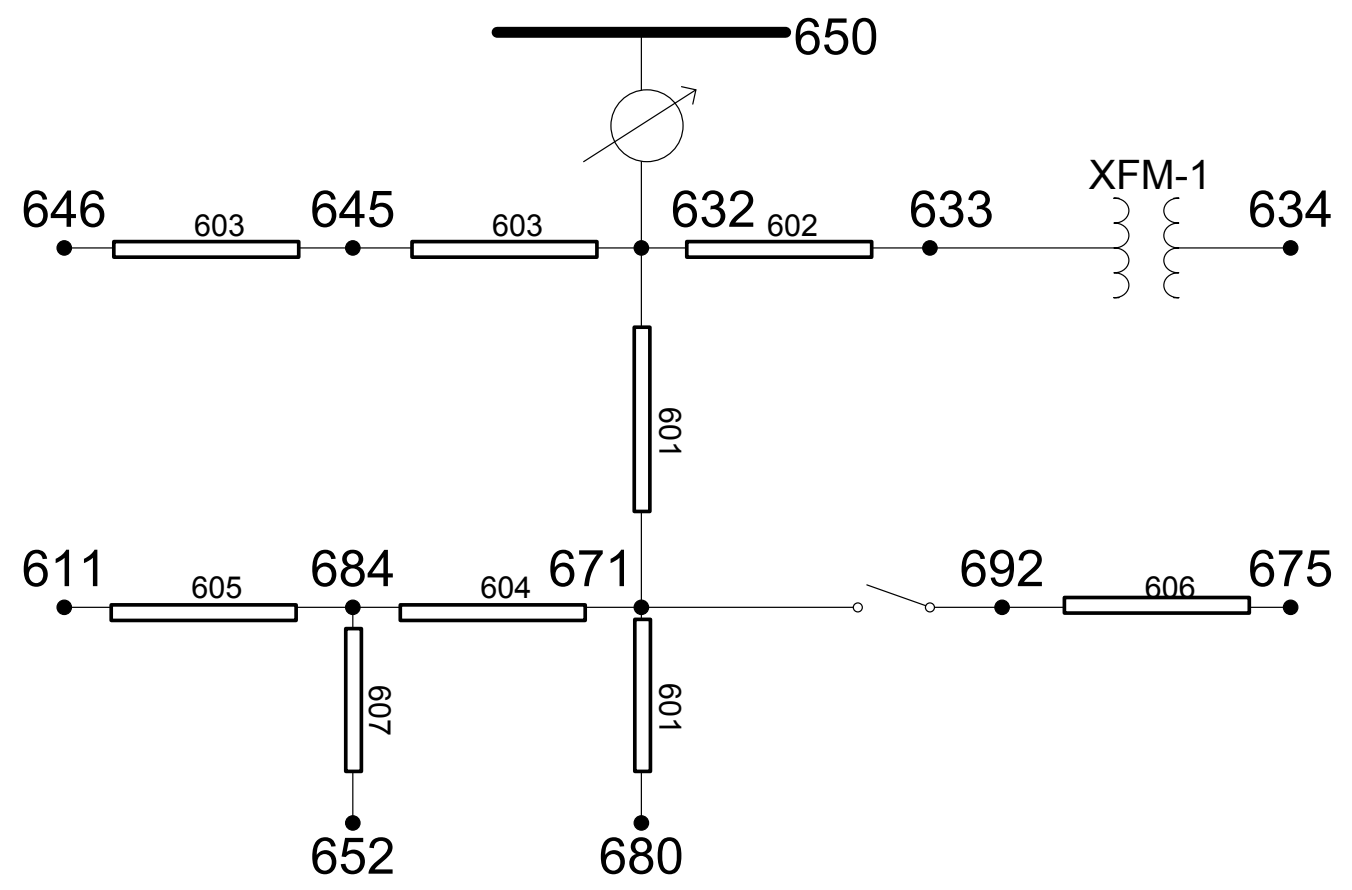

Fig. 2.10 Single line diagram of IEEE 13 Bus Feeder

The time domain modeling of IEEE 13 node feeder consists of three elements, distribution line modeling, load modeling and regulator modeling [97]. Seven different line type (601-67) are used in this system. Since line types 601, 602 and 606 are three phase, 603 and 604 are two phase and 605 and 607 are single phase, unbalanced line modeling is needed. There are four most commonly used types of load models: distributed load, constant power load, constant current load and constant impedance load. The existing transmission module libraries as well as voltage stability load models in PSCAD are not suited for distribution systems due to the load characteristics matching the four models discussed above. The available distribution PQ load model in PSCAD does not give accurate results as it uses a constant PQ load model to represent all kinds of loads as shown in [98]. Distribution level 
modeling and analysis have been presented in [99] for unbalanced power flow in steady state. The dynamic models for lines and loads for unbalanced IEEE 34 node feeder have been developed in $[99,100,101]$, However, the maximum absolute error of voltage magnitude and angle is higher than $2 \%$ and 2 degrees, respectively. This work develops models including unbalanced distribution lines, unbalanced spot and distributed loads which have not been previously developed. The same approach is used to model other IEEE feeders used in this work since the elements of distribution feeders are similar to IEEE 13 bus feeder. 


\section{Chapter 3. Problem Statement}

The major drawback of using STATCOM to mitigate the power quality issues related to PV integration is high cost. As the key component used to manage the power exchange between PV and power grid, a PV inverter is also a voltage source full bridge converter which is similar to a STATCOM. Hence PV inverters can be designed to perform STATCOM applications. Furthermore, traditional reactive power control doesn't incorporate active power control during system contingency, which results in overcharging of coupling capacitor bank and overshoot of active power output after fault clearance. Active power also needs to be regulated during fault to avoid increase of short circuit current. A multi-objective controller with Power Quality Control (PQC) and Fault Ride Through Control (FRTC) is proposed and tested on a 34 node system with a grid connected PV system under different simulation scenarios. A genetic algorithm approach is adopted to find the optimum PI parameters for the proposed controller. Generally, the PQC is activated to perform voltage flicker suppression, harmonic reduction, transient voltage support and unbalance compensation functions. During system contingency, FRTC improves the dynamic performance of PV generation by coordinating the controls of the PV power electronics interface, DC-DC booster and DC-AC inverter.

The intermittency of PV generation brings forth numerous challenges to system operation and design. These challenges result in variations in power flow, deteriorations in power quality and system stability, which are worsened by the increasing penetration levels of PV generation. To overcome these issues, storage devices such as batteries are considered as potential solutions to maintain the active power output levels of PV panels. The focuses on modeling and advanced control of PV farms and battery storage exploit the potential benefits of PV energy and battery. The transient stability and dynamic performance of the microgrid still needs further studies, and reactive power coordination between battery and PV generation has received little research attention. Insufficient reactive power compensation can cause voltage stability issues, which are worsened due to the remote location of PV farm. Battery can also provide dynamic reactive power support as well as provide instant active power supply when equipped with inverter interface that can be used to maintain voltage stability together with PV inverter. The modeling of a microgrid system with a Photovoltaic 
(PV) array and a battery storage system (BSS) is presented and a novel coordinated control scheme is designed to coordinate the PV system with battery inverters to provide high power quality to the grid. The proposed coordinated control scheme provides a comprehensive solution to both active and reactive power issues caused by the intermittency of PV energy and load changes, and it is tested in both a digital environment and RTDS-based hardware in the loop experimental platform.

In a standalone operation, a microgrid is isolated from the main AC supply system. Oscillations may occur during the transition results in voltage and frequency instability since synchronous alternators that can provide voltage and frequency references are not existed in the microgrid. An islanded control strategy with seamless transition ability is necessary for this situation. The operation and control design of a microgrid consisting of a direct drive wind generator and a battery storage system is proposed. A model predictive control strategy for the AC-DC-AC converter of a wind system is derived and implemented to capture the maximum wind energy as well as provide desired reactive power. A novel supervisory controller is presented and employed to coordinate the operation of a wind farm and battery system in the microgrid for grid-connected and islanded operation. The proposed coordinated controller can mitigate both active and reactive power disturbances that are caused by the intermittency of wind speed and load change. Moreover, the control strategy ensures the maximum power extraction capability of the wind turbine while regulating the PCC bus voltage within an acceptable range in both grid-connected and islanded operation. A modified seamless transient control will be presented to minimize the influence on system voltage and frequency during transient progress. The designed concept is verified through various simulation studies in EMTDC/PSCAD, and the results are presented and discussed.

The conventional coordinated control algorithm in microgrid works only around the designed operation point, however, due to high variability and uncertainty of renewable energies, the operation point of a power system with renewable resources is unpredictable, and the operating conditions continuously change in distribution networks. The integrated renewable resources should be able to adjust their outputs to track the changes in the operation conditions of the power system, indicating the dynamic regulation of renewable resources. A three-layer advanced optimization and intelligent control algorithm for a microgrid with multiple renewable resources is proposed. A Dual Heuristic Programming (DHP) based system control layer is used to ensure the dynamic reliability and voltage 
stability of the entire microgrid as the system operation condition changes. A local layer maximizes the capability of the Photovoltaic (PV), wind power generators and battery systems, and a Model Predictive Control (MPC) based device layer increases the tracking accuracy of the converter control. The proposed control scheme namely, System Wide Adaptive Predictive Supervisory Control (SWAPSC), reduces the influence of the intermittent nature of the renewable resources by smoothing the output of the PV and wind generators, maintains voltage stability by providing dynamic reactive power support to the grid, and reduces the total system loss as well as maximizes the usage of battery storages. The detail design is presented on an IEEE 13 node test system with a PV farm, a wind farm and two battery-based energy storage systems to demonstrate the proposed SWAPSC scheme. 


\section{Chapter 4. Power Quality Control}

The main component that connects the PV generator to the power system is a Voltage Source Inverter (VSI) which is similar to STATCOM. Therefore, the PV inverter can also perform voltage flicker suppression, harmonic reduction, and unbalance compensation as well as dynamic reactive power support.

\subsection{Feasibility Analysis}

The PV farm should generate as much active power as possible. The daily insolation profile shows that PV works under the rated capacity most of the time and hence the remaining capacity of the PV inverter can be utilized for power quality and system stability improvement. The decrease in active power due to control needs to be analyzed.

\subsubsection{Harmonic compensation}

For harmonic compensation, the required compensation capacity $S_{C h}$ of PV inverter can be calculated by

$$
S_{C h}=3 \sqrt{\sum_{n=1}^{\infty} U_{c n}^{2}} \times \sqrt{\sum_{n=1}^{\infty} I_{c n}^{2}}=3 \sqrt{U_{c}^{2} I_{c}^{2}}=3 S \times U_{T H D} \% I_{T H D} \%
$$

where, $S$ is the rated inverter capacity. $U_{c n}$ is the $n$-th harmonic voltage, $I_{c n}$ is the $n$-th harmonic current, $U_{c}$ is the vector sum of harmonic voltages, $I_{c}$ is the vector sum of harmonic currents. $U_{T H D}$ is the Total Harmonic Distortion (THD) of voltage waveform, $I_{T H D}$ is the THD of current waveform. Generally, $U_{T H D}$ is less than $10 \%$, and $I_{T H D}$ is less than $30 \%$. In the worst case scenario, assuming $U_{c}$ and $I_{C}$ are in phase, the compensation capacity required is only $3 \%$ of the rated inverter capacity.

\subsubsection{Unbalance compensation}

Likewise, required unbalance compensation capacity can be calculated by

$$
S_{C h}=3 \sqrt{U_{0}^{2}+U_{n}^{2}} \times \sqrt{I_{0}^{2}+I_{n}^{2}}
$$


In a three phase three wire distribution system, there are no zero-sequence current components, hence the equation (4.2) can be rewritten as

$$
S_{C h}=3 U_{n} \times I_{n}=3 S \times U_{\text {UnbalanceIndex }} I_{\text {UnbalanceIndex }}
$$

Generally, voltage and current unbalance indices are less than $5 \%$ and $30 \%$, respectively. In the worst case scenario, assuming $U_{c}$ and $I_{c}$ are in phase, the compensation capacity required is only $1.5 \%$ of the rated inverter capacity.

\subsubsection{Voltage flicker suppression and Dynamic reactive power support}

Voltage flicker suppression and Transient voltage mitigation can be achieved by reactive power compensation from the PV inverter. Neglecting switching losses there is no active power needed for reactive power support.

From sections 4.1, only a small amount of PV active power is utilized for compensation. Therefore, under a good control scheme, the PV inverter can be used for power quality and system reliability improvement.

\subsection{Power Quality Controller Design}

This section develops the multi-objective control strategy for the PV inverter to mitigate voltage flicker, harmonics, transient voltage and voltage unbalance associated with the PV system.

\subsubsection{Mitigation of voltage flicker}

Fig.4.1 shows the PV inverter control scheme to compensate voltage flicker. As seen in Fig.4.1, an $a b c-d q 0$ transformation as shown in equation (2.9) transfers three phase voltage to $d q 0$ reference frame at synchronous speed. $V_{q}$ is the component that follows the voltage oscillation of the network, while $V_{d}$ and $V_{0}$ are nearly zero. The transferred instantaneous vector $V_{q}$ is processed by low pass digital filter to extract $\mathrm{AC}$ sequence to finally obtain output signals $V_{s a b c}$. These signals are in turn used for calculating the conducting angle needed for triggering the IGBTs of the PV inverter. 


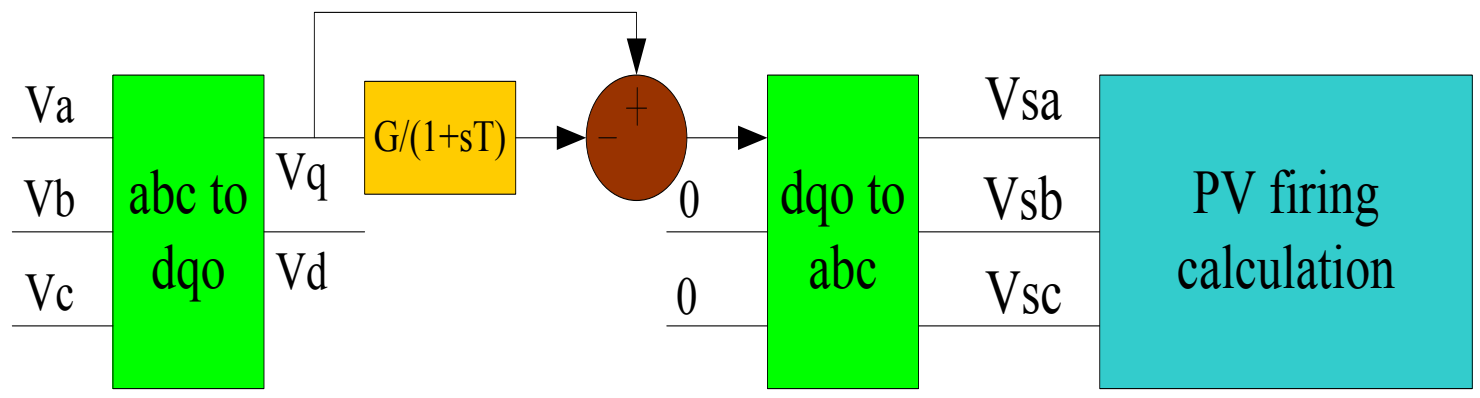

Fig.4.1 Flicker reduction control of PV inverter

\subsubsection{Harmonic compensator}

The proposed control scheme for harmonic reduction is as shown in Fig.4.2. An $a b c-d q 0$ transformation as shown in (2.9) transfers three-phase current to $d q 0$ reference frame whereby all the impurity sequences of $I_{d}$ and $I_{q}$ are eliminated by the cascaded low pass digital filters. A transformation to $a b c$ reference frame using (2.10) and processing in relation to original current $I_{a b c}$ results in $I_{h}$ which is superposed on the reference current of the inverter to obtain an output current similar in magnitude but opposite in direction of the system harmonic voltage.

By choosing $60 \mathrm{~Hz}$ as cutoff frequency, the first order low pass filter can approximately reduce $5^{\text {th }}$ harmonic to $20 \%$ and 7 th harmonic to $14 \%$. A filtering system consisting of two cascaded first order low pass filters can reduce $5^{\text {th }}$ harmonic to $4 \%$ and 7 th harmonic to $2 \%$.

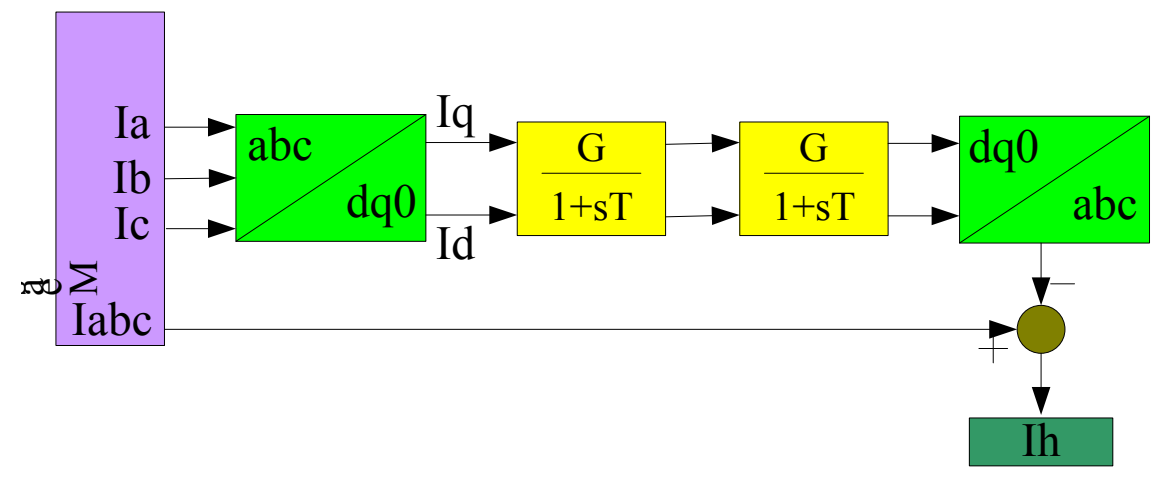

Fig.4.2 Harmonic Reduction Control

\subsubsection{Transient Voltage compensator}

Fig.4.3 shows the transient voltage regulation controller. As seen in Fig.4.3, the controller consists of an outer voltage control loop and an inner current control loop. $I_{d_{-} r e f}$ and $I_{q_{-} r e f}$ are obtained from the voltage control loop based on equations (4.4) and (4.5), 


$$
\begin{gathered}
P=\frac{3}{2}\left(E_{q} I_{q}+E_{d} I_{d}\right) \\
Q=\frac{3}{2}\left(E_{q} I_{d}-E_{d} I_{q}\right)
\end{gathered}
$$

where, $E_{d}$ and $E_{q}$ are the $d$ and $q$ components of system voltage, and $I_{d}$ and $I_{q}$ are $\mathrm{d}$ and q components of the PV inverter output current. Equations (4.4) and (4.5) give a representation of active power and reactive power of the PV inverter in synchronous $d q 0$ reference frame. Since $E_{q}$ is constant, and $E_{d}$ is equal to 0 at synchronous speed, equations (4.4) and (4.5) can also be written as,

$$
\begin{aligned}
& P=\frac{3}{2} E_{q} I_{q} \\
& Q=\frac{3}{2} E_{q} I_{d}
\end{aligned}
$$

It can be seen from equations (4.6) and (4.7) that the output power of the PV inverter can be controlled by regulating $I_{d \_r e f}$ and $I_{q_{-} r e f}$. Similar to equations (4.4) and (4.5), the relation between PCC voltage and PV terminal voltage in dq0 reference frame at synchronous speed can be derived as,

$$
\begin{aligned}
& E_{s d}=R I_{d}+p L I_{d}+L I_{q}+E_{d} \\
& E_{s q}=R I_{q}+p L I_{q}-L I_{d}+E_{q}
\end{aligned}
$$

where, $E_{s d}$ and $E_{s q}$ are the $d$ and $q$ components of the PV inverter terminal voltage, $L$ is the inductance of the line connecting PV node to PCC node. The final output signals $E_{s a b c}$ can be obtained from the inner current loop using $I_{d_{-} r e f}$ and $I_{q_{-r e f}}$ according to equations (4.8) and (4.9). These signals are in turn used for calculating the conducting angle, which is needed for triggering the IGBTs of the PV inverter. 


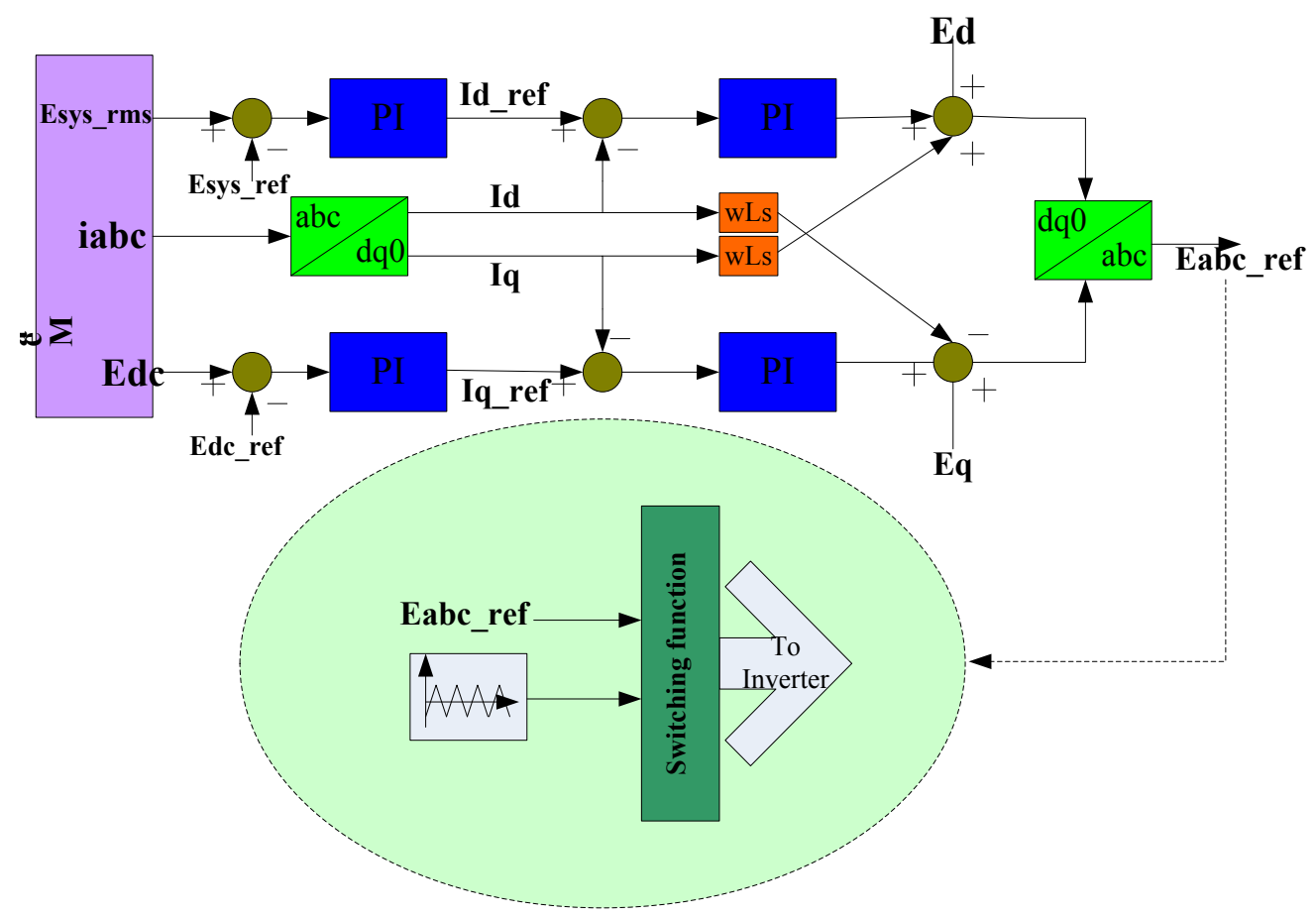

Fig.4.3 Internal control strategy for transient voltage regulation

An FRTC controller is designed to address transient voltage during system contingency. As shown in Fig.4.4, the FRTC coordinates the DC-AC inverter with the DC-DC booster so that during a fault if PCC voltage reduces to 0.7 p.u. for more than 2 cycles, the PV inverter output current $I d$ is limited to rated inverter current and the inverter output current $I q$ is calculated by $\left.\sqrt{(I m a x} x^{2}-I d^{2}\right)$. These limits on $I d$ and $I q$ maximize the reactive current and limit the active current during the fault; however, since there is active power generated by the PV panel and only a small amount of this power is delivered to the power grid, the surplus power charges DC capacitor bank and voltage $V_{d c}$ exceeds the acceptable level. The RMPPT control reduces the active power output of the PV panel by controlling the firing angle of the DC-DC booster to maintain the voltage within limits when voltage exceeds $1.03 \mathrm{pu}$. 


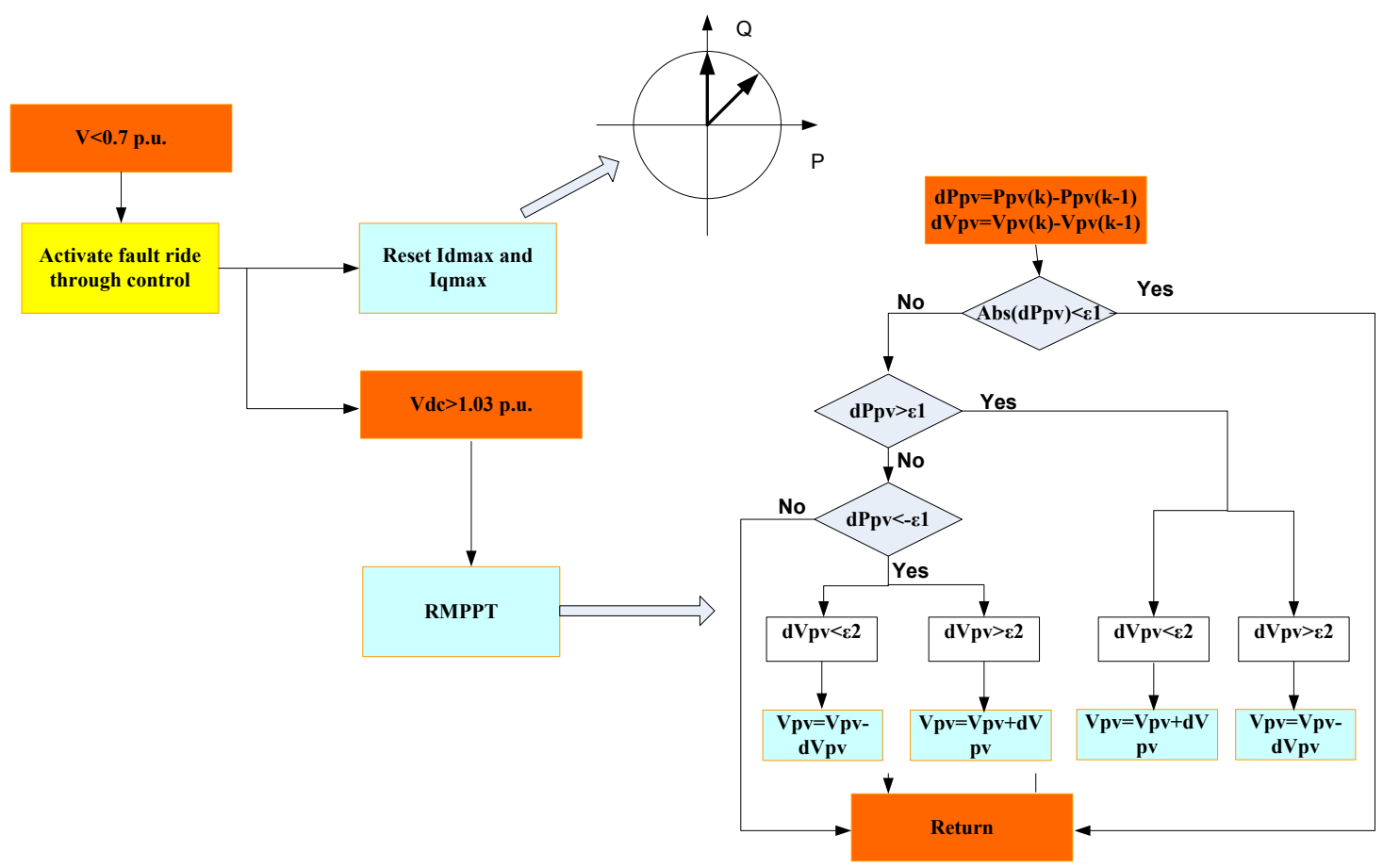

Fig.4.4 Fault Ride through Control

\subsubsection{Unbalance compensator}

Unbalanced load, unsymmetrical system fault or un-transposed lines can cause voltage unbalance. Among these causes, unbalanced load is the prominent cause in distribution networks. By compensating for unbalanced load at load terminal the system voltage and current unbalance can be reduced. An unbalanced signal can be decomposed to positive, negative and zero sequence by using symmetrical component decomposition method. The negative sequence component is the primary unbalance source in a three phase three wire system as zero sequence component doesn't exist. As shown in Fig.4.5, for the unbalance control scheme an output current similar in magnitude but opposite in direction of the negative sequence component is injected.

For this compensation an unbalance detection method similar to harmonic detection as shown in Fig.2.2 is required. However, equations (2.9) and (2.10) do not work effectively, especially when the system voltage is contaminated with harmonics. For compensation of negative sequence component, a new transformation matrix is needed. Considering negative sequence component in the reverse rotation of positive sequence, equations (2.9) and (2.10) can be rewritten as: 


$$
\begin{gathered}
{\left[\begin{array}{l}
q \\
d \\
0
\end{array}\right]=\frac{2}{3} \cdot\left[\begin{array}{ccc}
\cos (\theta) & \cos \left(\theta+\frac{2 \pi}{3}\right) & \cos \left(\theta-\frac{2 \pi}{3}\right) \\
\sin (\theta) & \sin \left(\theta+\frac{2 \pi}{3}\right) & \sin \left(\theta-\frac{2 \pi}{3}\right) \\
\frac{1}{2} & \frac{1}{2} & \frac{1}{2}
\end{array}\right] \cdot\left[\begin{array}{l}
a \\
b \\
c
\end{array}\right]} \\
{\left[\begin{array}{l}
a \\
b \\
c
\end{array}\right]=\left[\begin{array}{ccc}
\cos (\theta) & \sin (\theta) & 1 \\
\cos \left(\theta+\frac{2 \pi}{3}\right) & \sin \left(\theta+\frac{2 \pi}{3}\right) & 1 \\
\cos \left(\theta-\frac{2 \pi}{3}\right) & \sin \left(\theta-\frac{2 \pi}{3}\right) & 1
\end{array}\right] \cdot\left[\begin{array}{l}
q \\
d \\
0
\end{array}\right]}
\end{gathered}
$$

The negative sequence $I_{n}$ in $a b c$ frame is obtained by the inverse transformation after eliminating the impurities using the cascaded low pass digital filters. The control scheme to compensate voltage unbalance is shown in Fig.4.5.

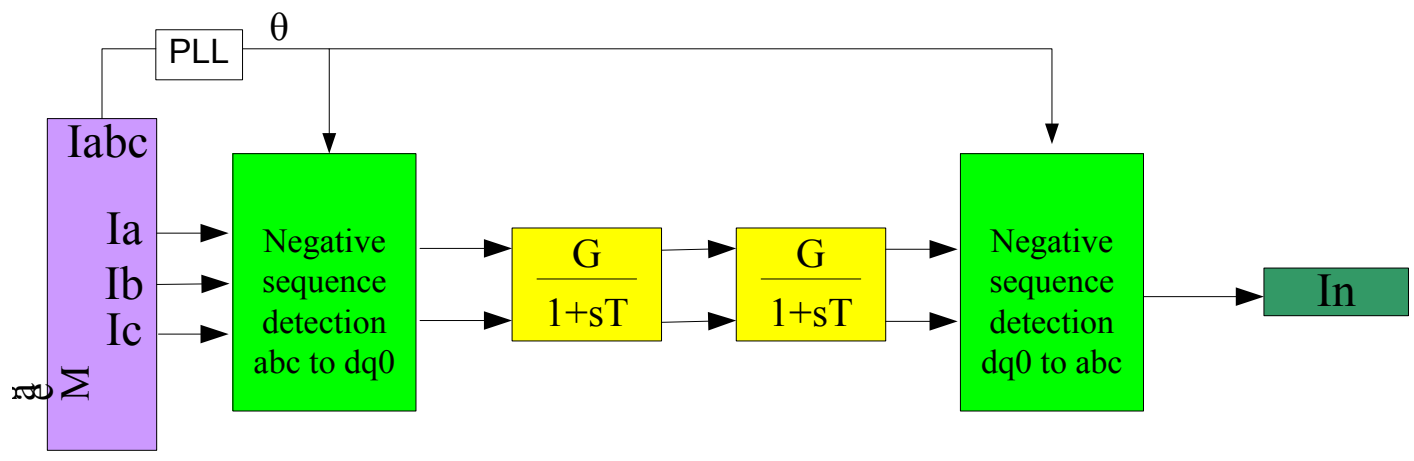

Fig.4.5 Unbalance Compensation Control

\subsection{Simulation Results}

In order to verify the proposed control strategy, a detailed PSCAD model of PV generator is developed. The PV generator is connected at node 890 of the IEEE 34 node system as shown in Fig.4.6. The tap change set points of the regulators PT1 and PT2 are $122 \mathrm{~V}$ and $124 \mathrm{~V}$, respectively, and the deadband is $\pm 1 \mathrm{~V}$. Several scenarios are simulated and the results are presented in this section. 


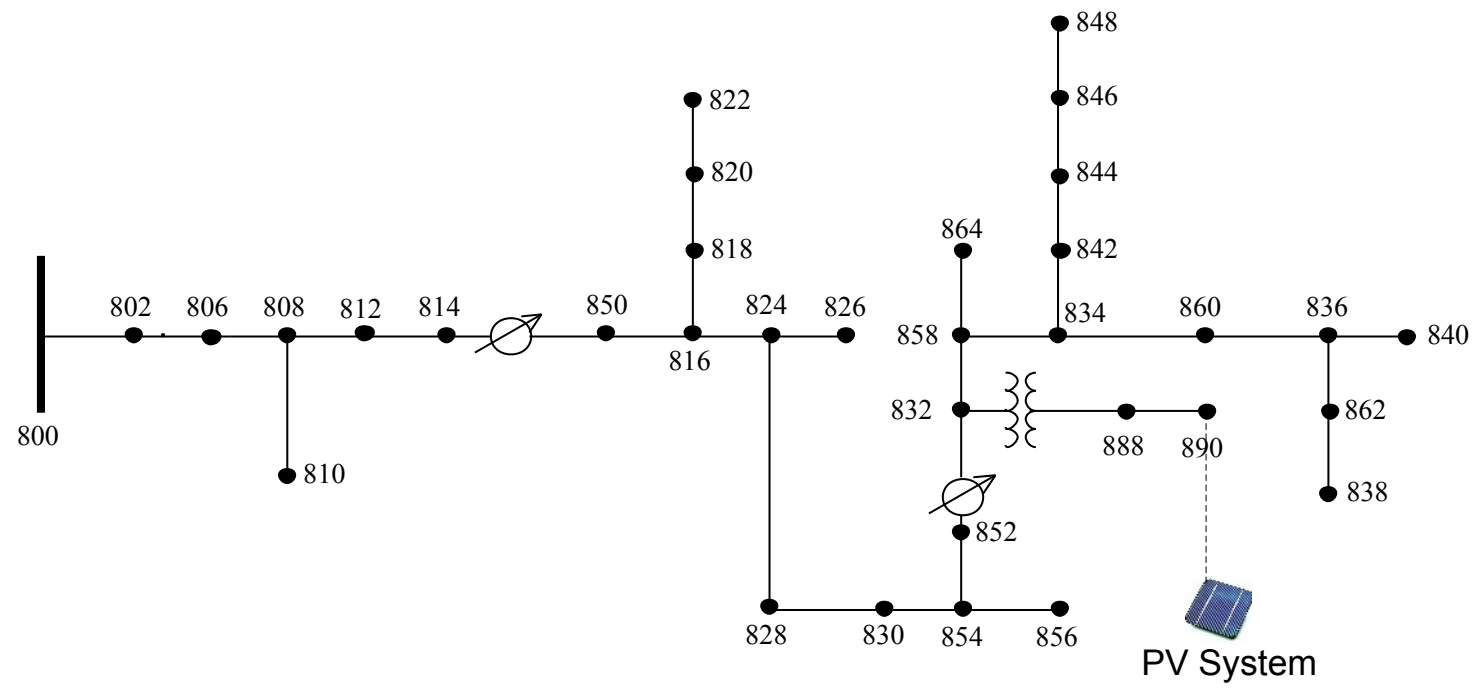

Fig.4.6 IEEE 34 Nodes system with PV integration

\subsubsection{Mitigation of Voltage Flicker}

A mutative irradiation is given as an input to the PV panel. The base value of the irradiation is $800 \mathrm{~W} / \mathrm{m}^{2}$ with a $5 \mathrm{~Hz} \pm 200 \mathrm{~W} / \mathrm{m}^{2}$ deviation in the interval $2.2 \mathrm{~s}-2.8 \mathrm{~s}$, as shown in Fig.4.7. Fig.4.8 shows the output power of PV where without voltage flicker control no reactive power support is provided by PV farm and therefore, the active power is lower than maximum value due to the low voltage level. However, deploying power quality control reactive power compensates for the voltage variations and active power tracks the maximum power point during insolation changes. Fig.4.9 shows RMS value of the PCC voltage with and without power quality control. It is seen that without voltage flicker control there are large voltage variations from the steady state voltage level of 0.93 p.u. with the lowest being 0.82 p.u. at 1.47s due to the rapid changes of PV active power. These fluctuations cause flicker in system voltage. However, with the power quality control developed in 4.2.1, the system voltage is maintained at $1 \pm 0.03$ p.u. in steady state and during rapid changes of PV active power, demonstrating the effectiveness of the control strategy. 


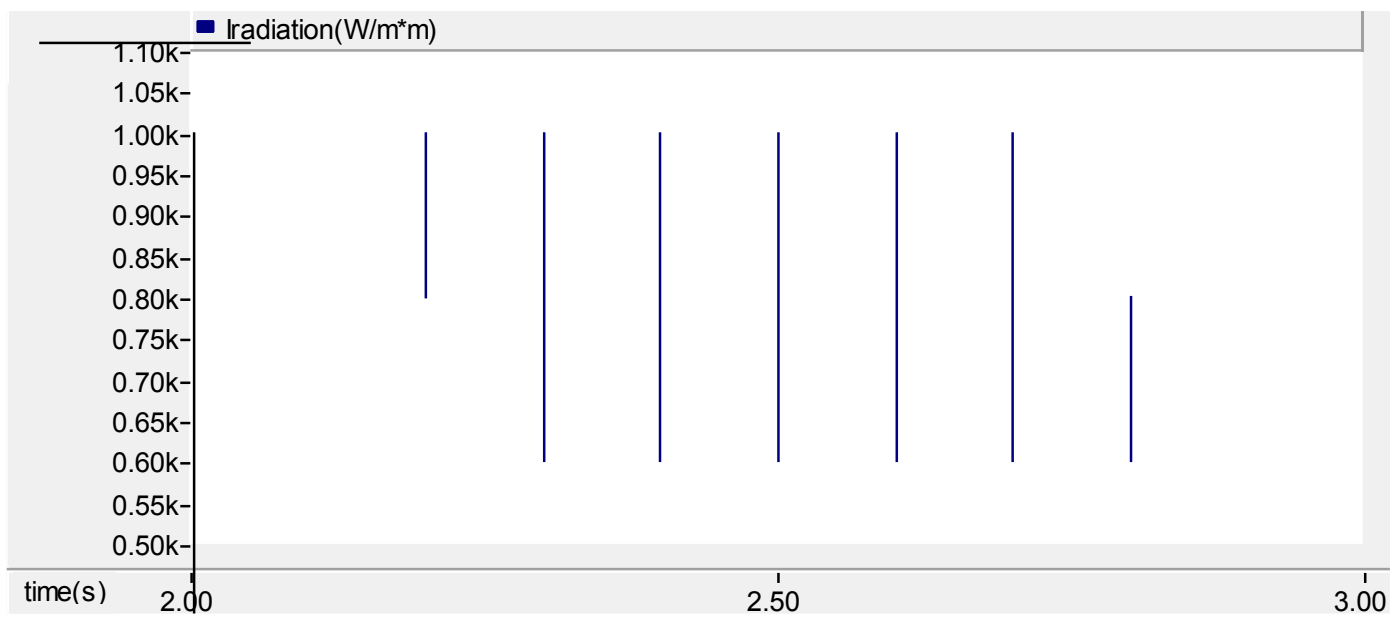

Fig.4.7 Input irradiation of PV cell

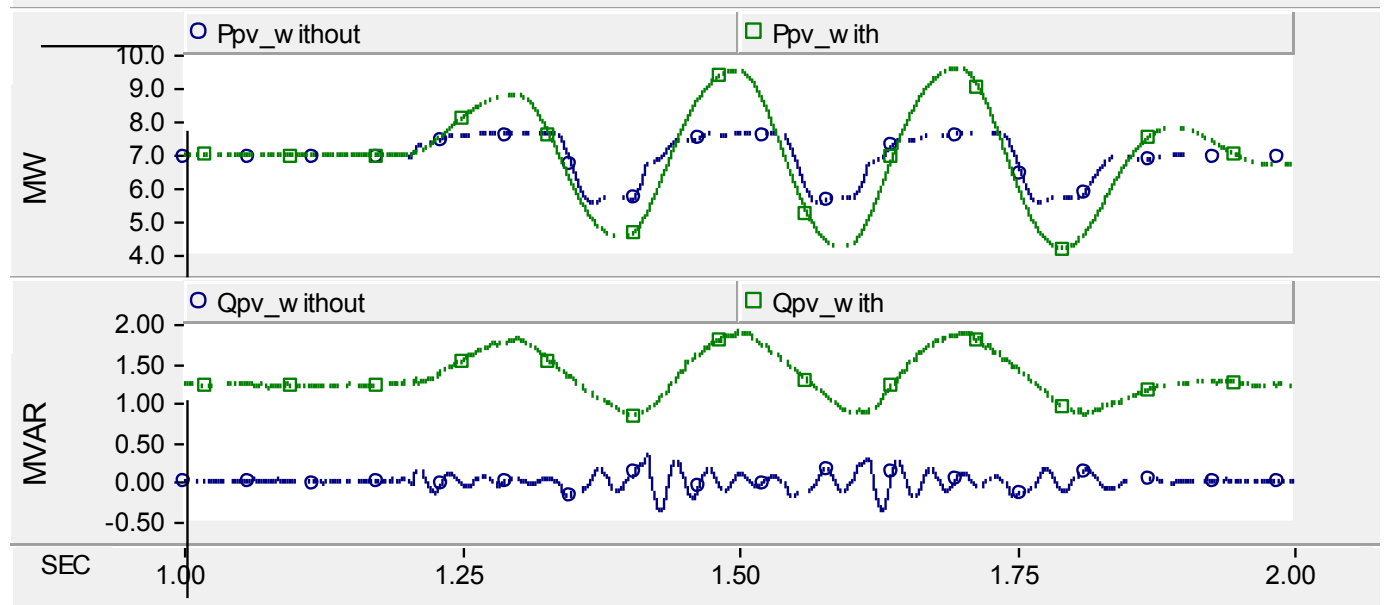

Fig.4.8 PV output power to the grid

$00000:$ PV active power output;

$\square \boxminus \boxminus \boxminus \boxminus:$ PV reactive power output 


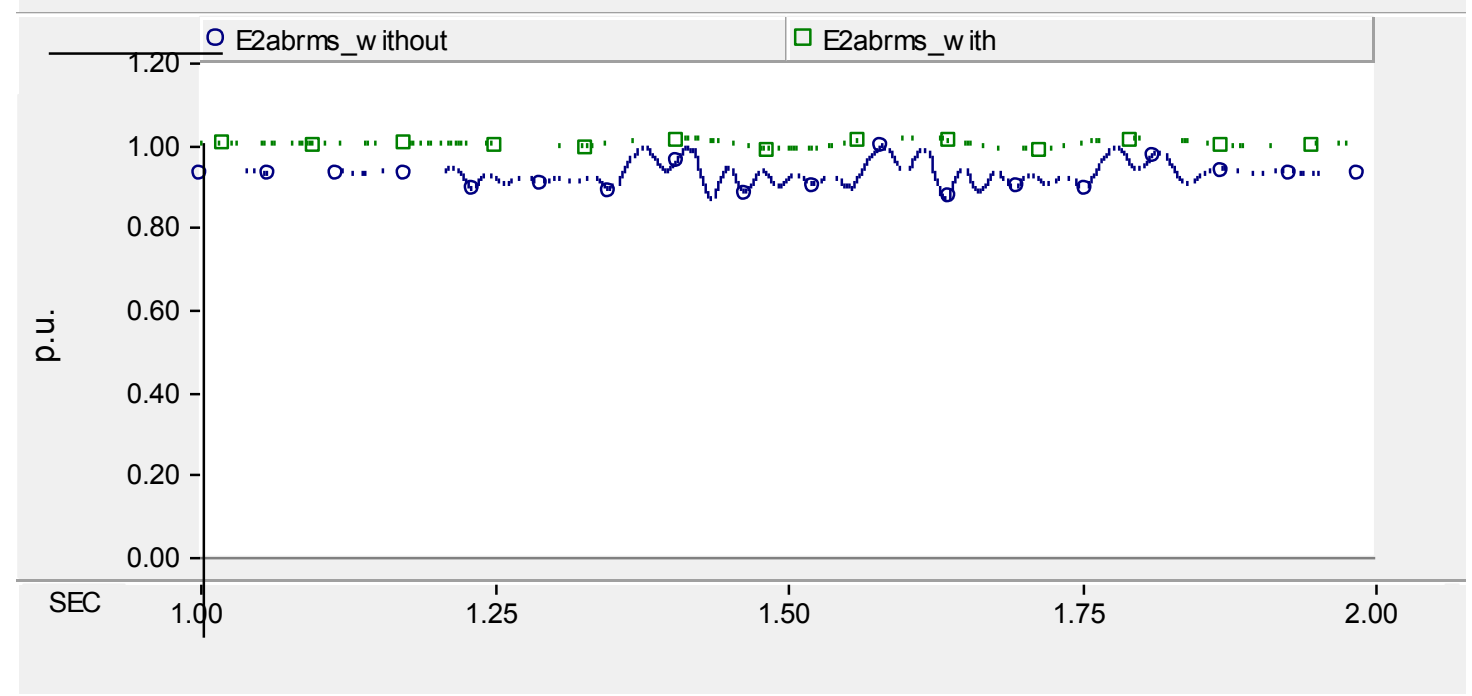

Fig.4.9 Grid side line voltage RMS value

00000 : System line voltage RMS value without voltage flicker control;

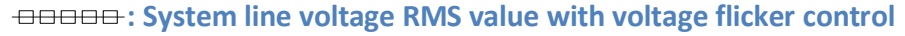

\subsubsection{Harmonic compensation}

The rating of $\mathrm{PV}$ generator is $0.7 \mathrm{MW}$ at $1000 \mathrm{~W} / \mathrm{m}^{2}$. Harmonic compensation strategy as shown in 4.1.2 is applied. For the harmonics of orders $5^{\text {th }}$ and $7^{\text {th }}$ with amplitudes $100 \mathrm{~A}$ and $50 \mathrm{~A}$ respectively are simulated by the current source. The insolation level is maintained at $1000 \mathrm{~W} / \mathrm{m}^{2}$. The MPPT control is activated at $0.3 \mathrm{~s}$, and the harmonic reduction control is activated at $0.5 \mathrm{~s}$.

Fig.4.10 shows the voltage $E_{b e}$ before and voltage $E_{a f}$ after the LC filter. $E_{b e}$ is a square wave, which is filtered using the filter designed in 5.1, to sinusoidal wave $E_{a f}$. This shows that the PV generator is not acting as source of harmonics and on the contrary it injects harmonics for mitigation. Also, the power is maintained at maximum power point as shown in Fig.4.12 by increasing $V_{\text {mppt }}$ from $0.3 \mathrm{kV}$ to $0.328 \mathrm{kV}$. The voltage and current ripples are less than $1 \%$ and 5\% respectively. 


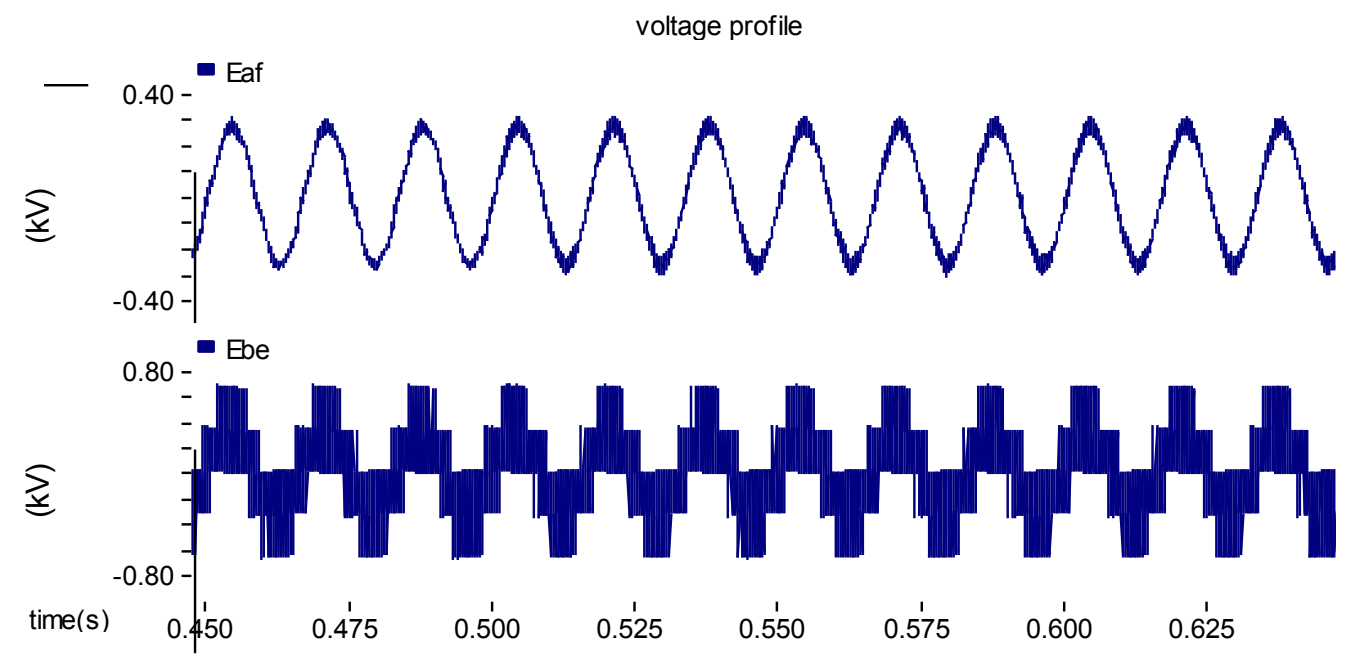

Fig.4.10 Voltage at the terminals of the Filter

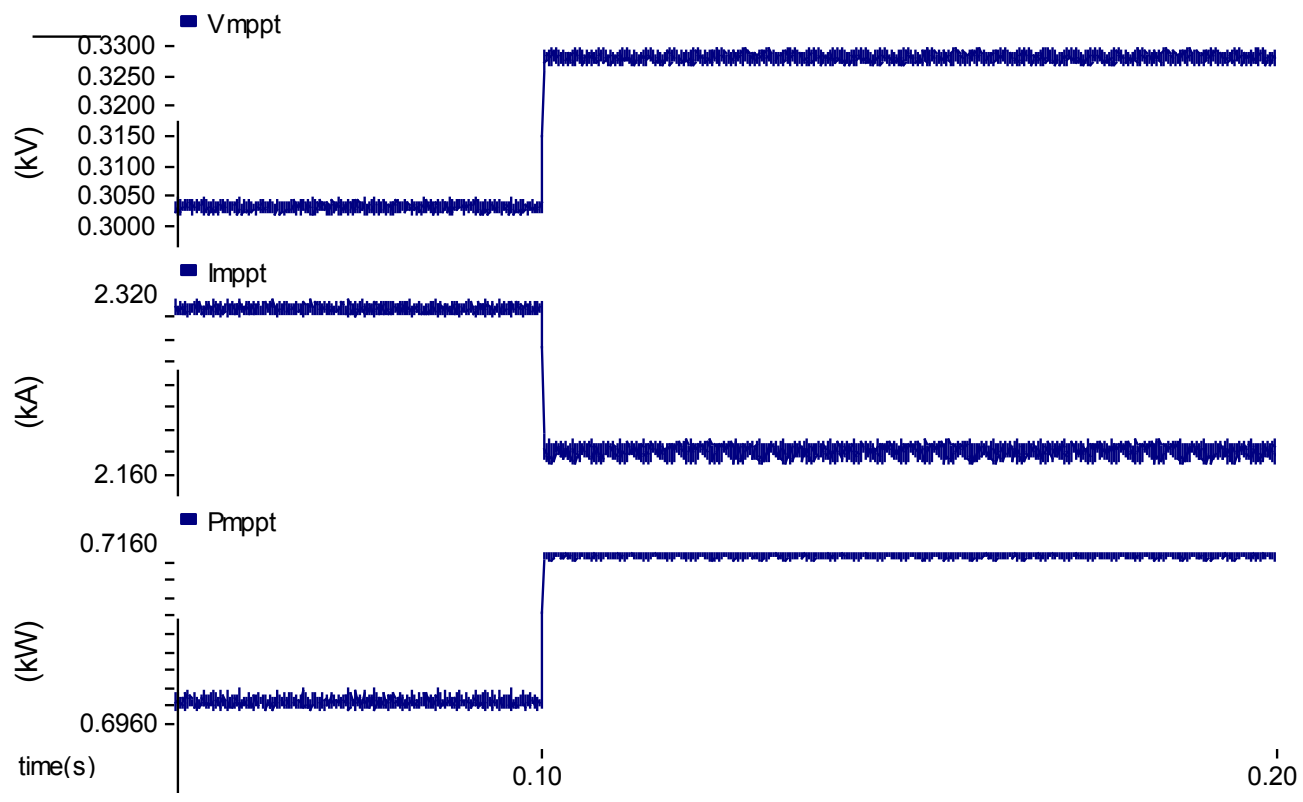

Fig.4.11 PV panel Characteristics with MPPT 


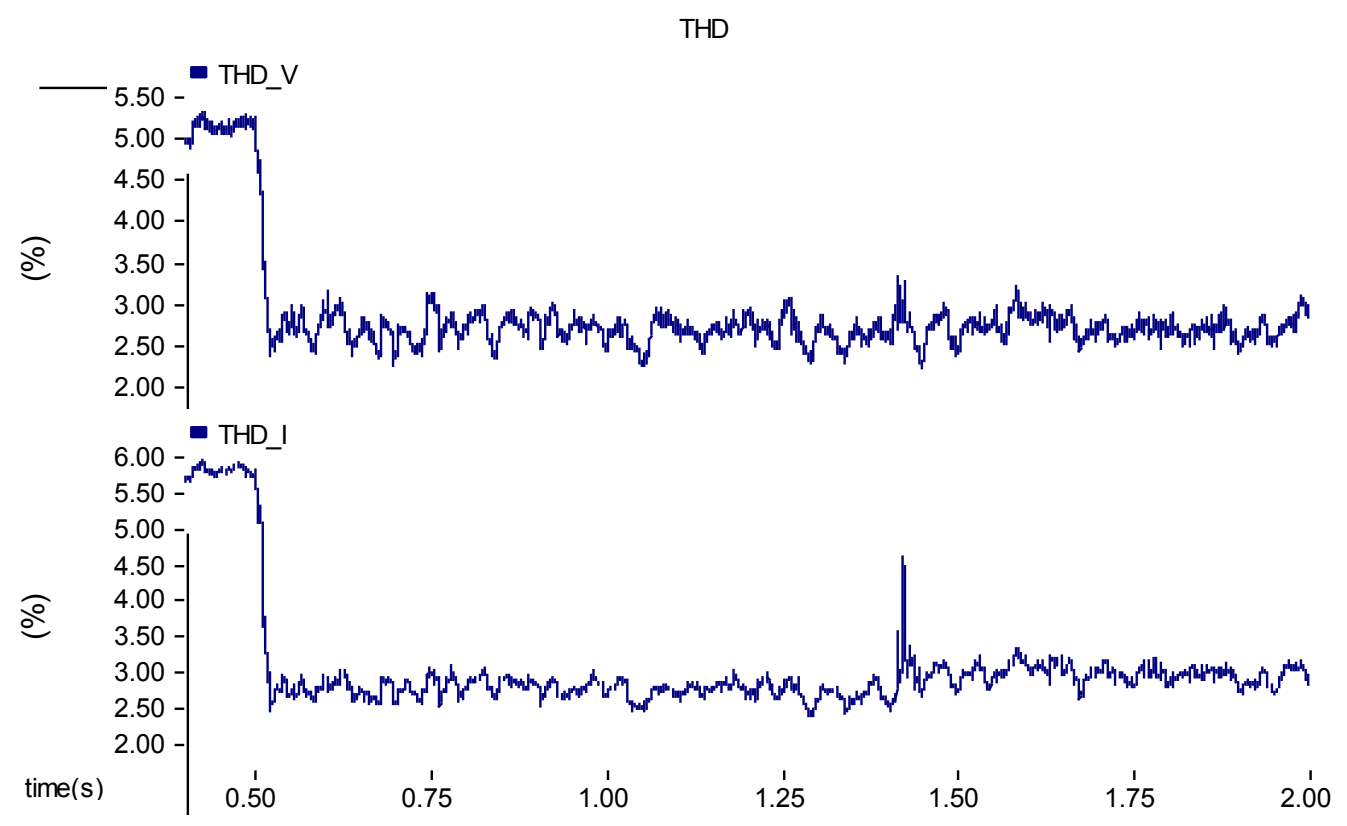

Fig.4.12 THD of Voltage and Current Waveform of PV farm

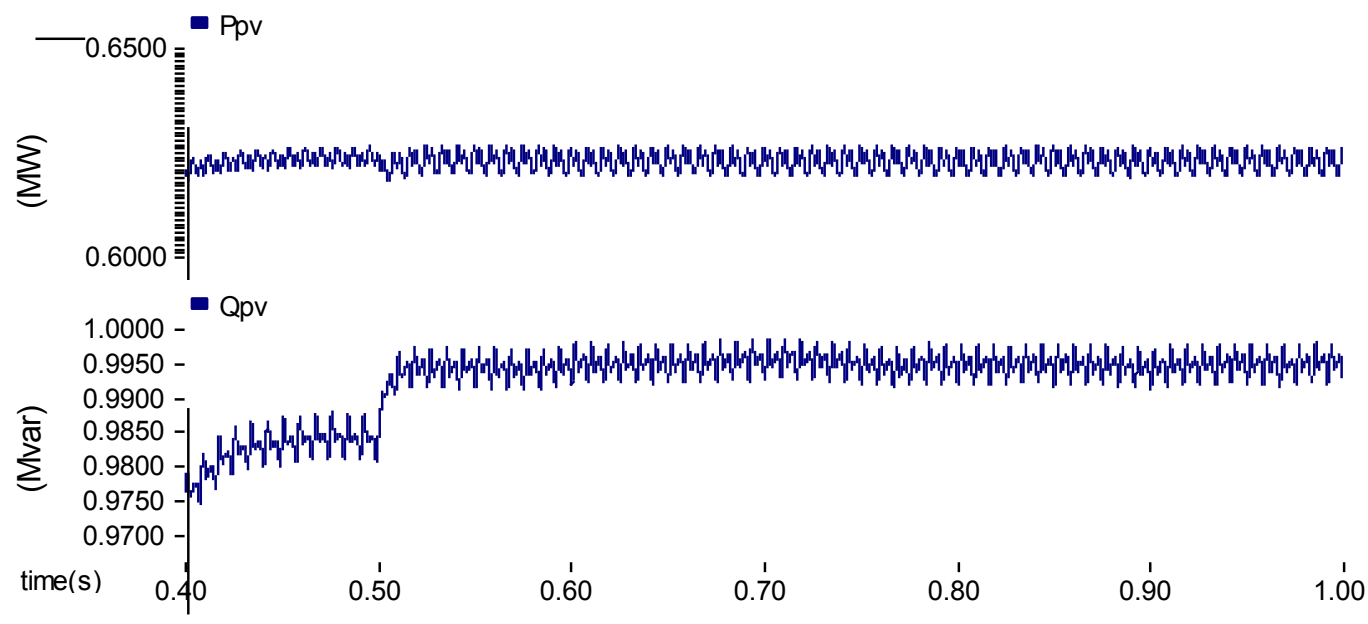

Fig.4.13 Output power of PV farm during Harmonic Compensation

The THD of voltage and current waveforms of the PV farm are shown in Fig.4.12. It can be seen that $T H D_{-} V$ is around $5.2 \%$ before the compensation and according to IEEE standards, this $T H D_{-} V$ exceeds the acceptable levels. However, after compensation the $T H D_{-} V$ decreases significantly and the maximum $T H D_{-} V$ is lesser than 3\% while the $T H D_{-} I$ is reduced from 5.8\% to $2.6 \%$ at $0.5 \mathrm{~s}$. A corresponding result is the increase of reactive power of $\mathrm{PV}$ inverter during the harmonic compensation and little change in active power of PV farm as shown in Fig.4.13. 


\subsubsection{Mitigation of transient voltage}

The rating of PV generator is $9 \mathrm{MW}$ at $1000 \mathrm{~W} / \mathrm{m}^{2}$. A three-phase fault with an impedance of $1 \mathrm{ohm}$ occurs near the PCC at 1.0s and it is cleared at 1.1s. It is seen from Fig.4.14 that without FRTC, the active power is not supplied during fault, overshoots after fault is cleared and takes 0.65 s to reach steady state due to DC capacitor overcharge as shown in Fig.4.16. With FRTC, the active power is not supplied during fault, overshoots less after fault is cleared and takes 0.083 s to reach steady state due to constant DC capacitor bank voltage.

As shown in Fig.4.14, without FRTC, the reactive power is not supplied by the power electronic inverter during steady state and 4 MVAR reactive power is supplied on fault clearance as a portion of the inverter capacity is used to generate active power. Without FRTC, the PCC bus voltage changes from 0.93 p.u. in steady state to $0.2 \mathrm{p} . \mathrm{u}$ during fault as shown in Fig.4.15. However, with FRTC, reactive power is supplied during steady state and increases to 8MVAR after fault clearance. With FRTC, PCC bus voltage changes from 1 p.u. in steady state to 0.2 p.u. during fault.

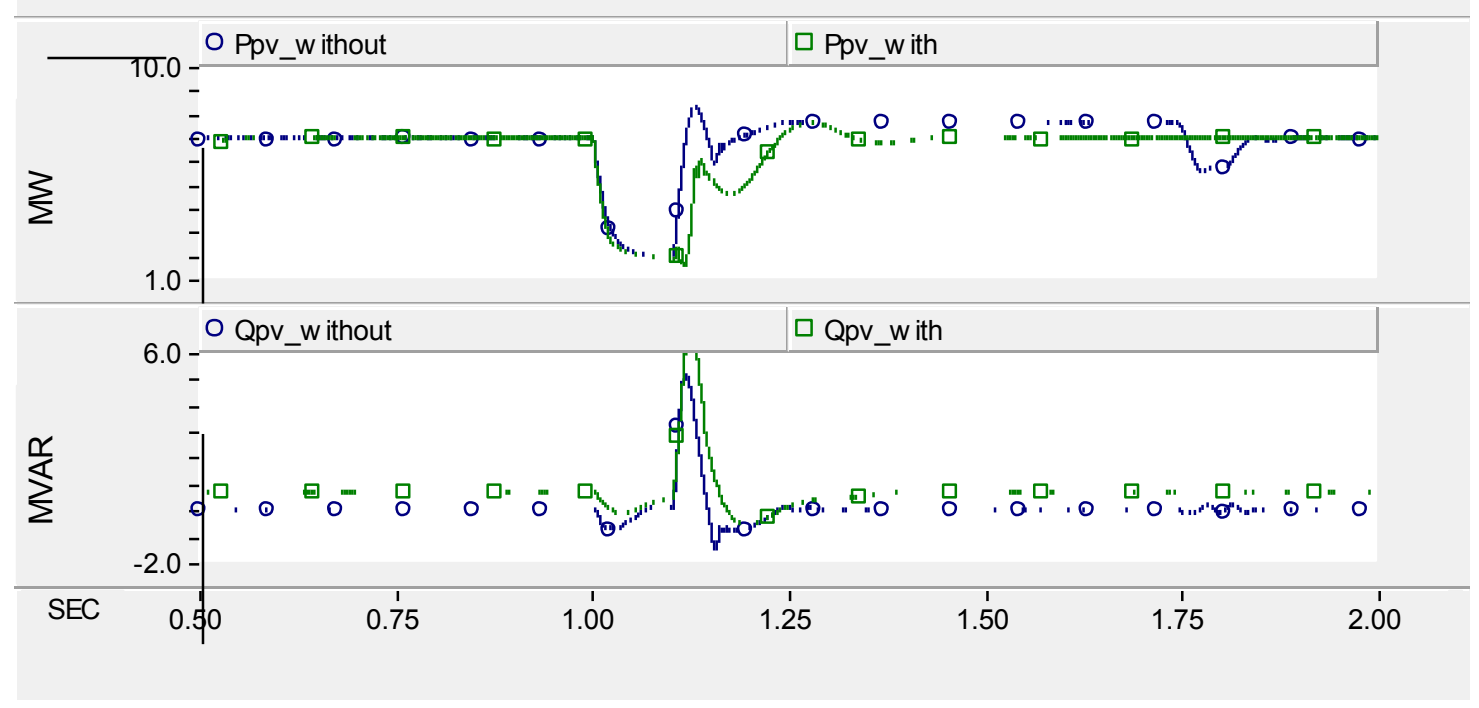

Fig.4.14 PV generation and PCC bus voltage 


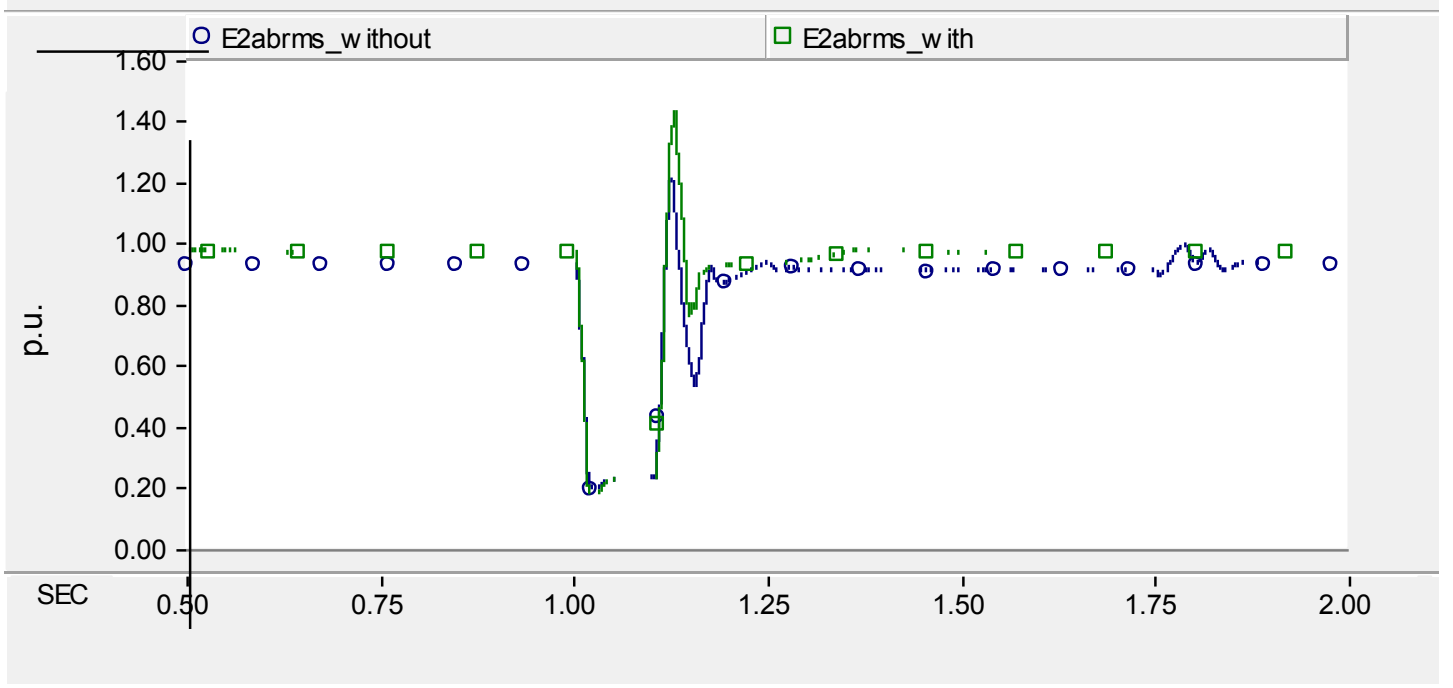

Fig.4.15 PCC bus voltage RMS value

-0000: Without FRTC

$\square \square \square \square \square:$ With FRTC

\begin{tabular}{l|l}
\hline & Edc_with \\
\hline
\end{tabular}

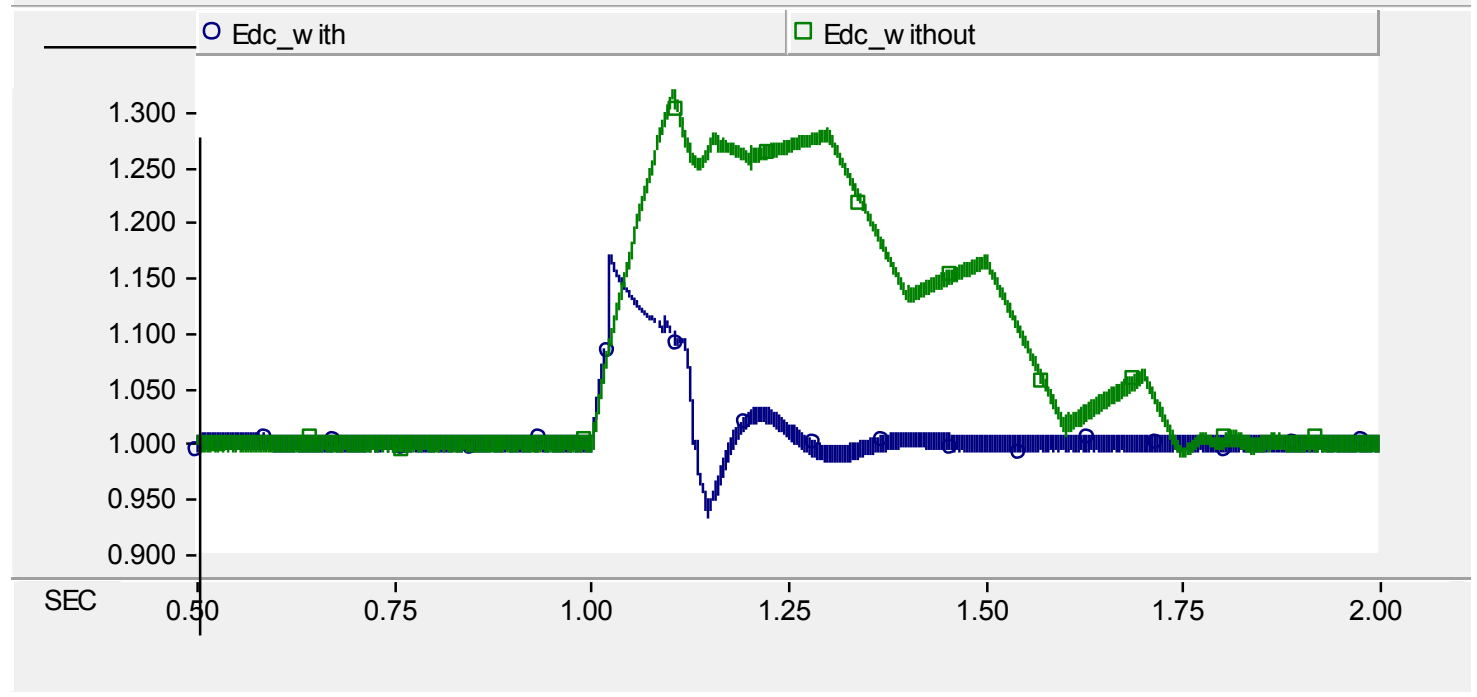

Fig.4.16 DC capacitor bank voltage

$00000:$ Without FRTC

$\square \square \square \square \square:$ With FRTC

Fig.4.17 shows the terminal voltage of PV generator with and without FRTC control. It is seen that the terminal voltage during fault is very high without FRTC. However, with FRTC, the terminal voltage is maintained zero during system fault. 


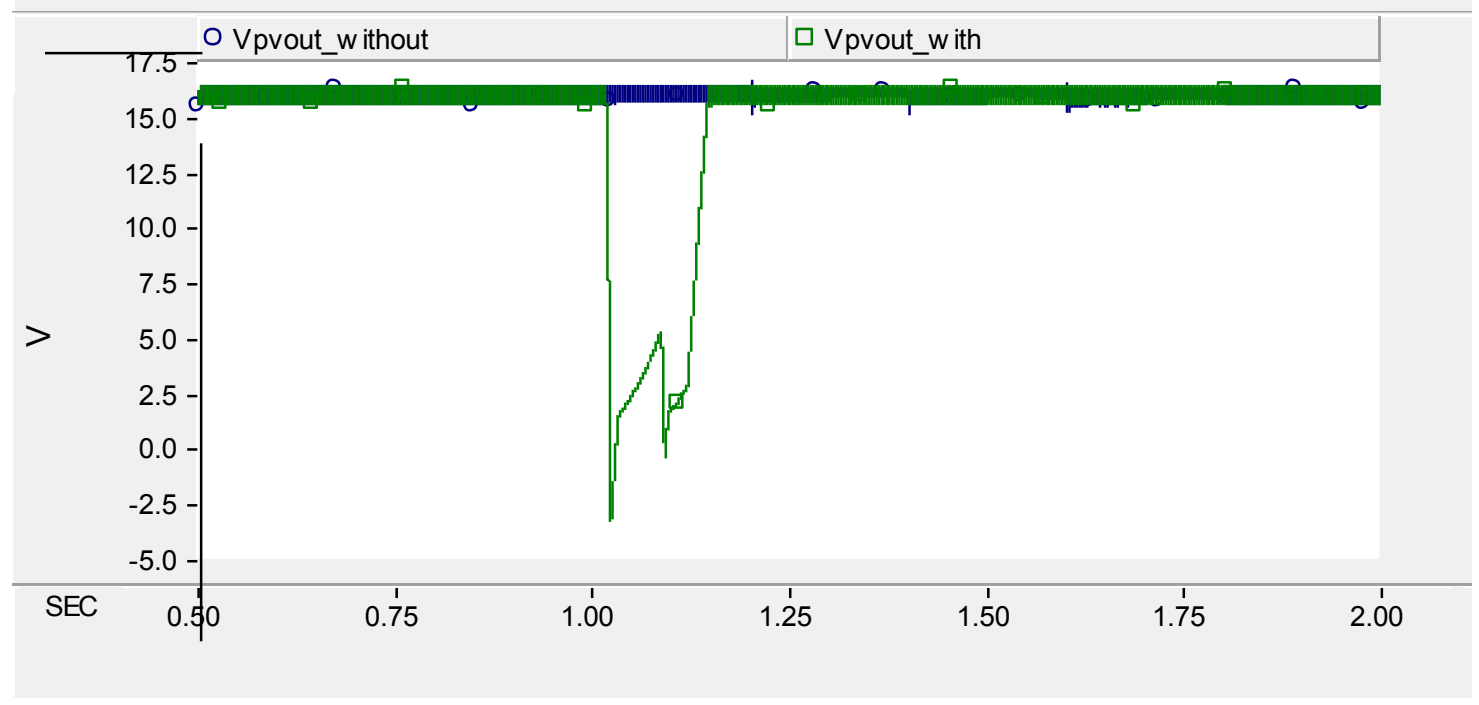

Fig.4.17 The terminal voltage of PV generator

00000 : Without FRTC

$\square \square \square \square \square:$ With FRTC

\subsubsection{Unbalance compensation}

The rating of $\mathrm{PV}$ generator is set as $0.7 \mathrm{MW}$ at $1000 \mathrm{~W} / \mathrm{m}^{2}$. Unbalance compensation strategy discussed in section 4.1 .2 is applied and activated at $0.5 \mathrm{~s}$ simulation. A three phase unbalanced load is connected to node 846. The load ratings in phases $A, B$ and C are 0.17 MVA with 0.95 lag, 0.12 MVA with 0.97 lag, and 0.23 MVA with 0.8 lag respectively. 


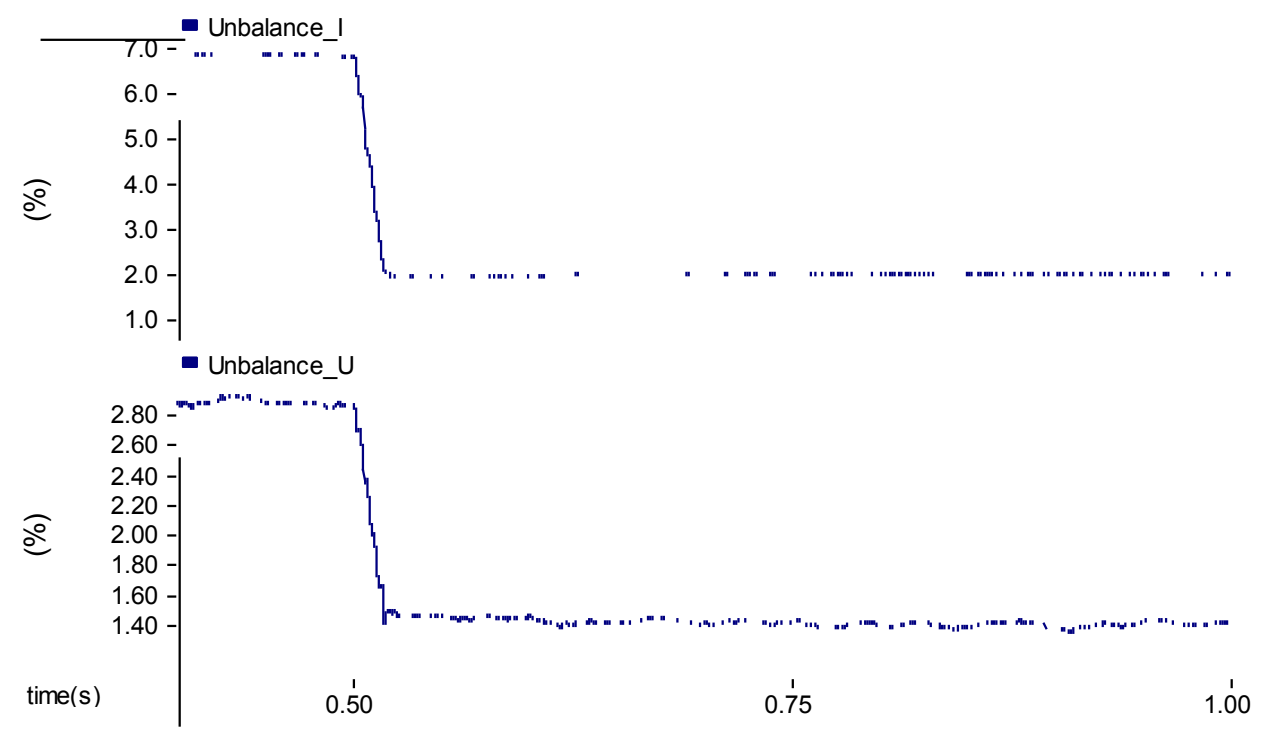

Fig.4.18 Unbalanced indices of PCC Voltage and Current

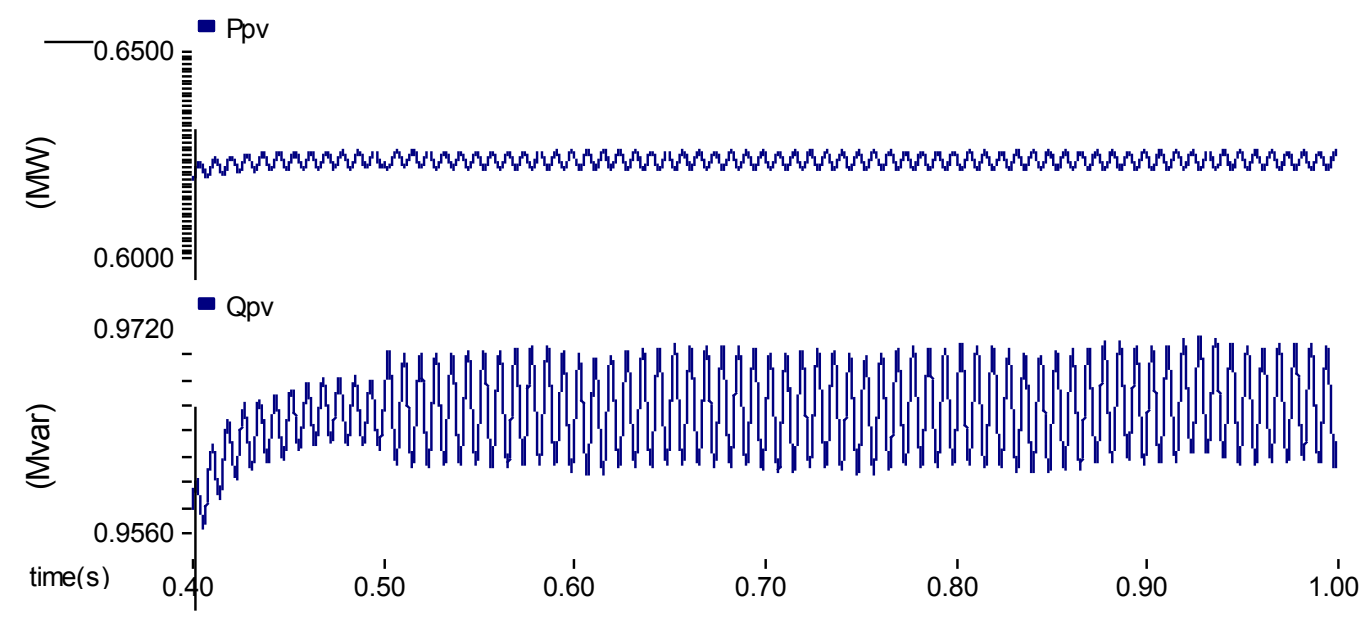

Fig.4.19 Output power of PV farm during Unbalance Compensation

Fig.4.18 shows the system voltage and current unbalance indices before and after compensation. It is seen that the PCC voltage and current unbalance indices are reduced and more balanced after the compensation. Moreover, the active power output as shown in Fig.4.19 doesn't change much due to the compensation. 


\subsection{Chapter Conclusion}

This section develops a multi-objective control scheme for a grid connected three phase PV system. The simulation result is done in PSCAD and the result show that this control scheme can perform voltage flicker suppression, unbalance compensation as well as harmonic reduction. Moreover, the control scheme also improves the fault ride through ability of PV farm by improving the dynamic performance of the power electronic interface during a system fault.

This control scheme can also been used in direct drive wind turbine since the power electronic interface of DDWT is similar to PV inverter. 


\section{Chapter 5. Modeling and Coordinated Controller Design of A PV/Battery System}

To design and test a coordinated controller, a transient system model with highly accurate representation of the complexity of the PV panel and battery unit is needed. Fig.5.1 shows the one line diagram of the microgrid system consisting of a weak power grid, a PV array system, a battery storage system, and two different kinds of loads: a fixed impedance load and a controllable fixed power load.

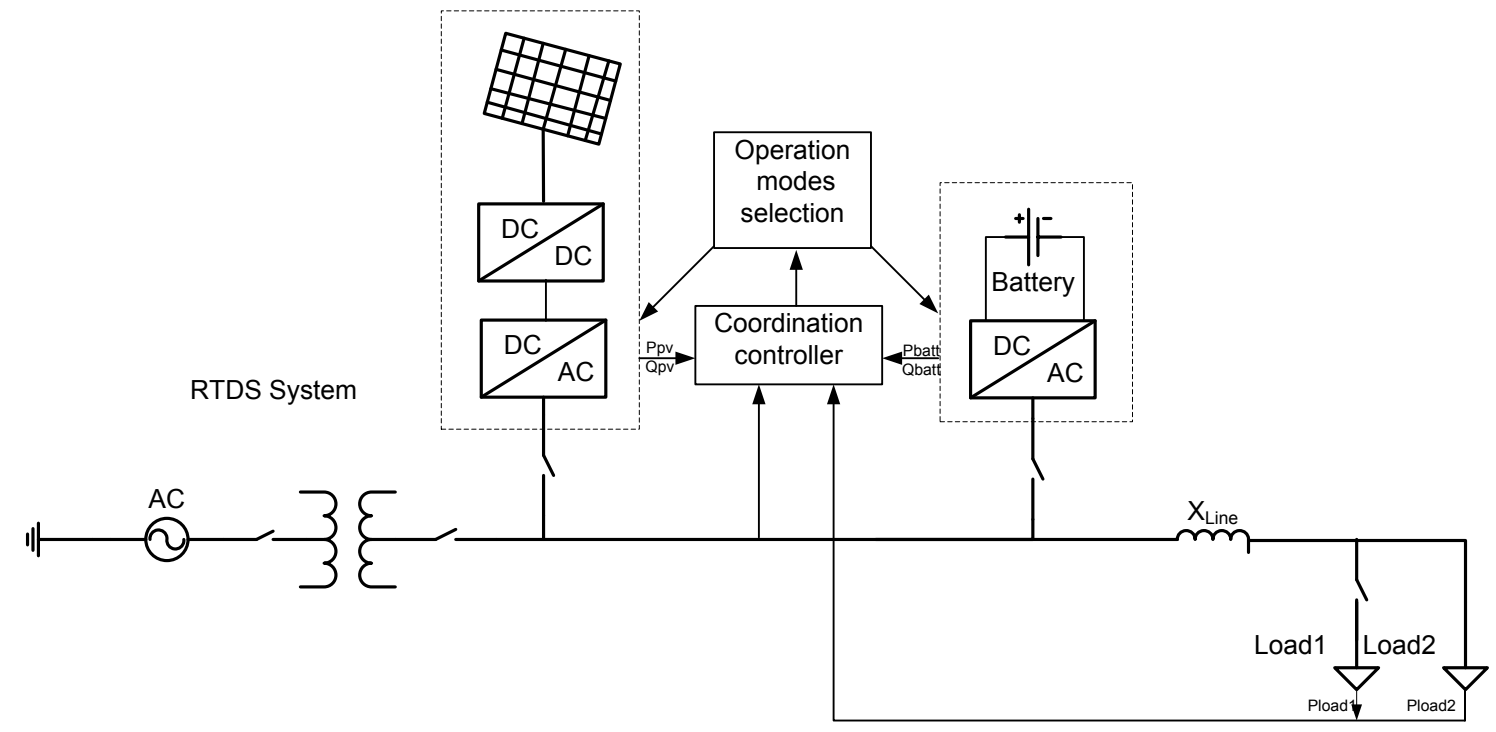

Fig.5.1 One line diagram of the hybrid system

\subsection{Circuit design}

The PV system is connected to the grid through its power electronic interface including DCDC booster and DC-AC inverter. The selections of circuit components such as $L, C_{\text {in }}$ and $C_{\text {out }}$ in DC boost circuit and $L_{f}, C_{f}$ in the inverter circuit as shown in Fig.5.2 are essential to the control strategy. 


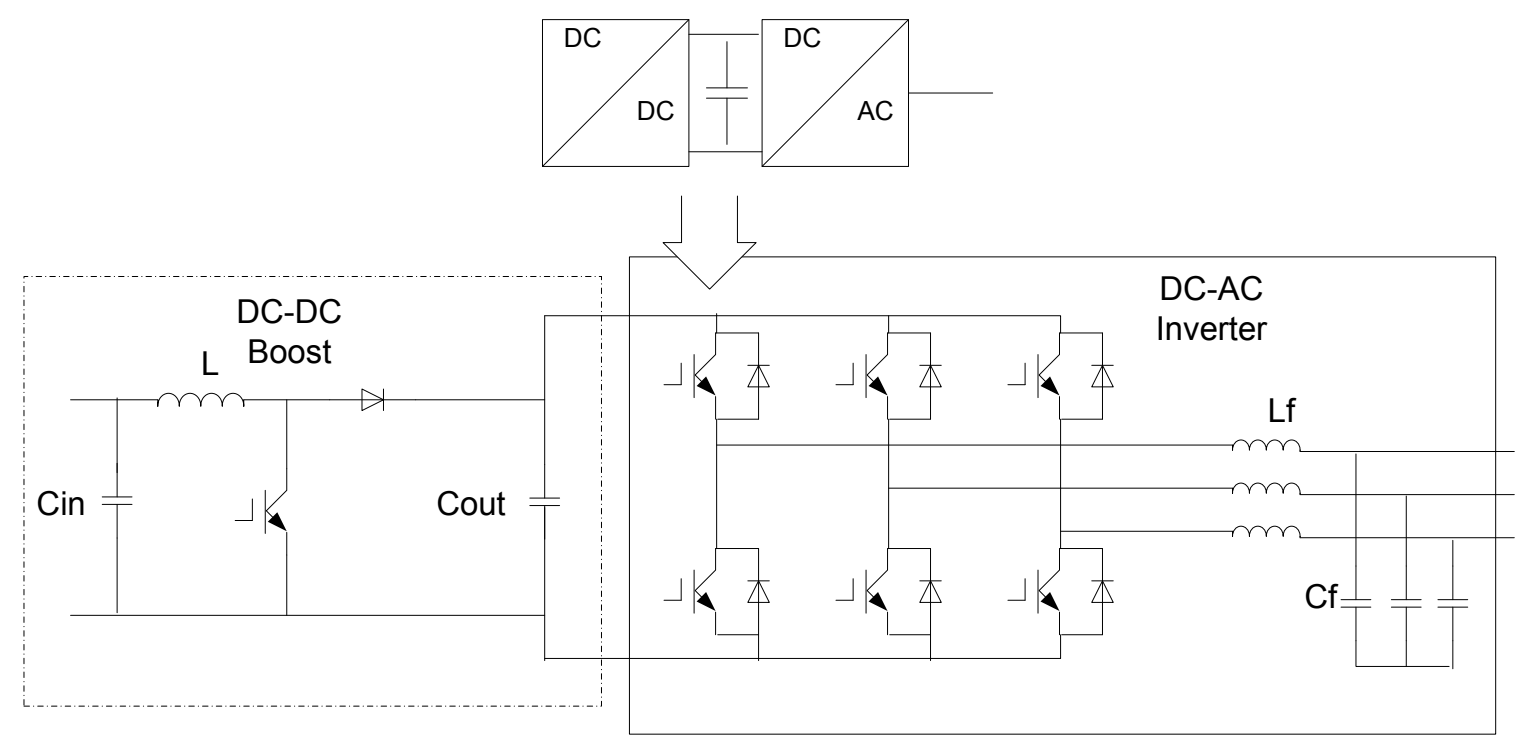

Fig.5.2 Power electronics interface of PV farm

The inductor $L$ in the boost converter is used to limit the current ripple $\Delta i_{L}$ through the inductor and the semiconductor devices such as diode and thyristor. The inductor $L$ is so chosen that $\Delta i_{L}$ is not too small leading to an increase in inductance, and hence the switching loss and cost. Also, $\Delta i_{L}$ cannot be too large leading to an increase in the peak current of the inductor, and hence size and cost. Typical values of $\Delta i_{L}$ are in the range of $10 \%$ to $20 \%$ of the rated current. In this work, the current ripple $\Delta i_{L}$ is selected as $10 \%$ and $L$ is given by:

$$
L=\frac{V_{o} \times D(1-D) \times T_{S}}{\Delta i_{L}}
$$

$T_{S}$ is the switch cycle, and $D$ is the duty cycle given by (5.2)

$$
\frac{V_{o}}{V_{i}}=\frac{1}{1-D}
$$

where, $V_{o}$ is the output DC voltage of the booster, and $V_{i}$ is the PV panel terminal voltage. The input capacitor $C_{i n}$ is used to restrain the voltage ripple at the PV panel terminal and hence yield better voltage profile and MPPT performance. $C_{i n}$ can be calculated as

$$
C_{i n}=\frac{\Delta i_{L} \times T_{S}}{8 \Delta V_{c i n}}
$$

where, $\Delta V_{\text {cin }}$ is the voltage ripple at the PV panel terminal. Typical values of $\Delta V_{\text {cin }}$ lie in the range of $1 \%$ to $5 \%$. 
The voltage ripple $\Delta V_{\text {cout }}$ at the capacitor output is given by

$$
\Delta V_{\text {cout }}=\frac{V_{o} D T_{S}}{R C_{\text {out }}}
$$

where, $R$ is the equivalent load if the load is purely resistive. $C_{\text {out }}$ can be calculated using $\Delta V_{\text {cout }}$ from equation (5.5)

$$
C_{\text {cout }}=\frac{V_{o} D T_{S}}{R \Delta V_{\text {cout }}}
$$

The LC filter design is based on voltage ripple and current ripple analysis. The voltage ripple and current ripple cannot be too small, which would weaken the harmonic reduction ability and cannot be too large which would lead the PV inverter to become a harmonic source. Here, $\Delta V_{a c L}$ is set as $0.05 V_{a c L}$ and $\Delta i_{a c}$ is set as $0.1 i_{a c}$. With specified maximum ac current ripple $\Delta i_{a c(\max )}$, the value of $L_{f}$ can be calculated from equation (5.6).

$$
\Delta i_{a c(\max )}=\frac{0.433}{3} \times \frac{V_{o}}{L_{f}} \times T_{S}
$$

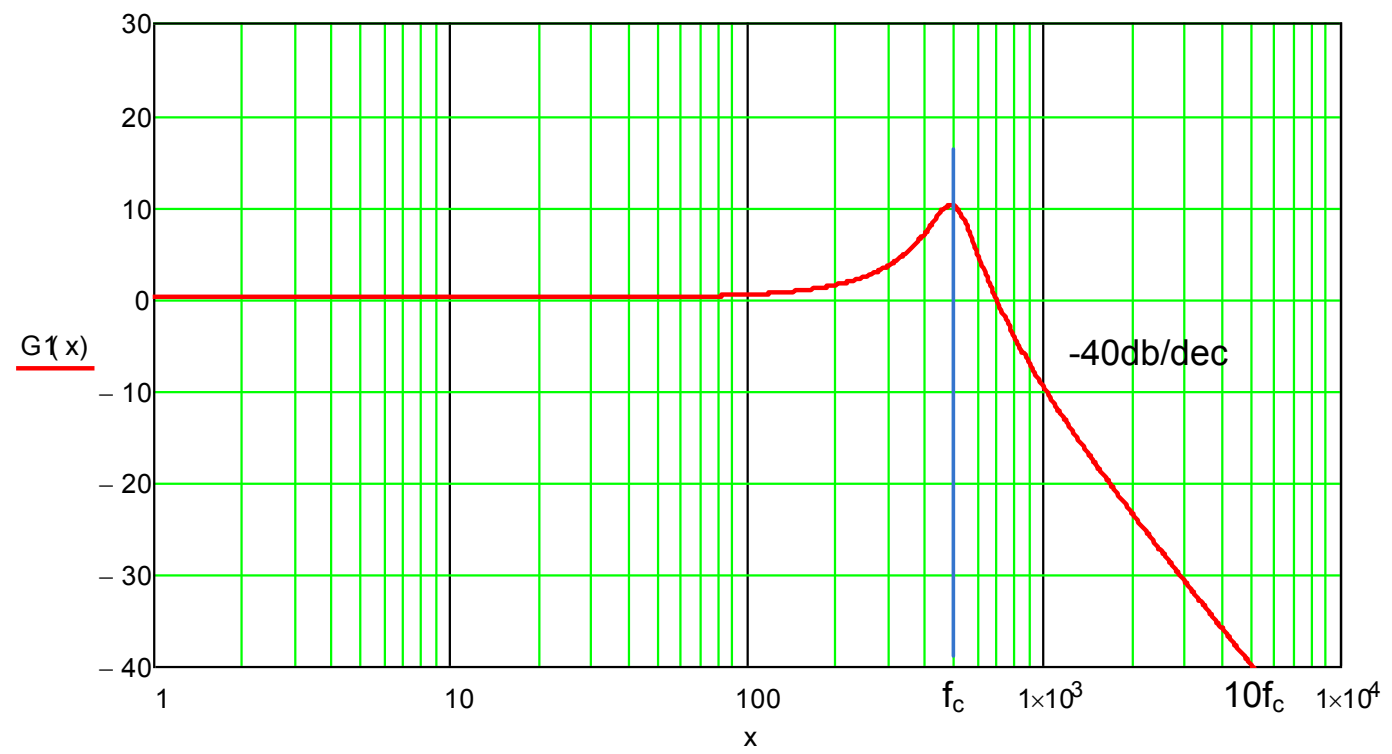

Fig.5.3 Gain versus frequency curve of a second-order LC low- pass filter

The inductors and capacitors compose a second-order filter for which the gain vs. frequency curve is shown in Fig.5.3. The corner frequency $f_{c}$ is equal to

$$
f_{c}=\frac{1}{2 \pi \sqrt{L_{f} C_{f}}}
$$


The gain of the LC low-pass filter yields

$$
\operatorname{gain}(f)=\left|\frac{\Delta V_{a c L}}{V a c L}\right|=\left|\frac{1}{1+\frac{L_{f}}{R}\left(2 \pi f_{s} i_{a c}+L_{f} C_{f}\left(2 \pi f_{s} i_{a c}\right)^{2}\right.}\right|=\left|\frac{1}{1+Q\left(i_{a c} \frac{f_{S}}{f_{c}}\right)+\left(i_{a c} f_{\frac{S}{f_{c}}}\right)^{2}}\right|
$$

where, $\Delta V_{a c L}$ is the ac voltage ripple, $i_{a c}$ is the ac current, $f_{s}$ is the switching frequency, $Q$ is the quality factor. When $f_{s}$ is far greater than the corner frequency, the gain is approximately equal to

$$
\operatorname{gain}(f) \approx\left(\frac{f_{s}}{f_{c}}\right)^{2}=\left|\frac{\Delta V_{a c L}}{V a c L}\right|
$$

\subsection{PV Electronic Interface Design}

\subsubsection{PV DC-DC boost control}

There is always a single maximum power point of the PV generator for each operating condition and it is desired that the PV generator operate at this point. The DC voltage of the PV inverter should be higher than the magnitude of the PCC line to line voltage. The PV DCDC booster controller as shown in Fig.5.4 maintains the PV panel operation at maximum operating points and higher voltage level of the DC bus of PV inverter, higher than the magnitude of the PCC line to line voltage.

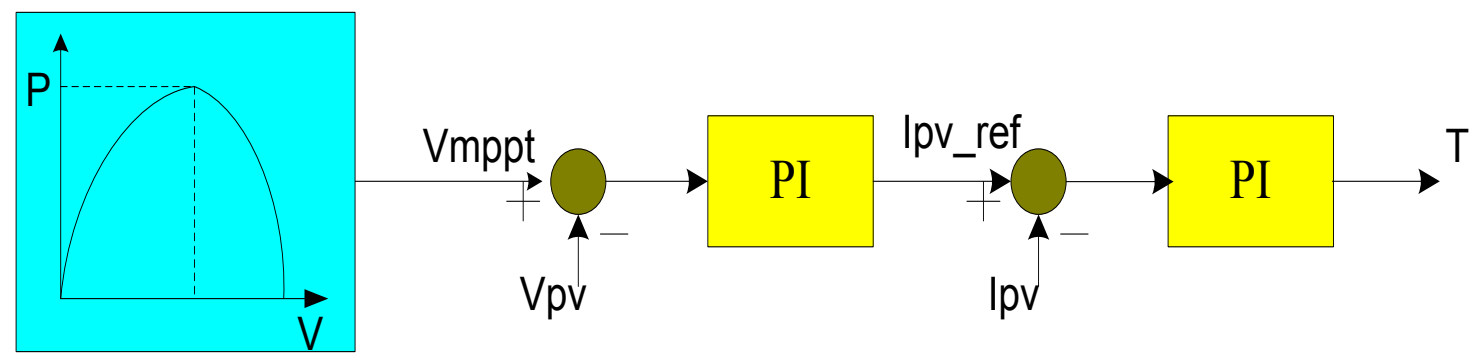

Fig.5.4 PV DC-DC booster controller

A Perturbation \& Observation (P\&O) MPPT technique is implemented to obtain voltage command at maximum power point Vmppt.Ipv_ref is used to trace the voltage error between Vmppt and actual voltage Vpv through the outer voltage loop and the output signal $T$ for the triggering can be obtained from the inner current loop. The inner current loop improves the current as well.

\subsubsection{PV DC-AC and battery inverter control}


Real power generated by the PV panel is regulated to deliver maximum power to the grid while maintaining the voltage of the coupling capacitor between the PV DC-DC booster and the PV DC-AC inverter. The active and reactive power regulation is achieved by the Voltage Controlled Current Source (VCCS) inverter control as shown in Fig.5.5. A dq0 transformation is implemented to obtain the decoupled control of the active power and reactive power output for the PV inverter. The $q$-axis and $d$-axis current commands Iqpv_ref and Idpv_ref are obtained through the PI controller to regulate active and reactive power output respectively. $I_{\text {modulation }}$ is used to determine the angle needed for triggering the IGBTs of the PV inverter. Reactive power regulation has two modes: Zero Reactive Power Control (0-RPC) mode and voltage control mode. Optimal dynamic power support for different system scenarios is achieved by switching between these two modes.

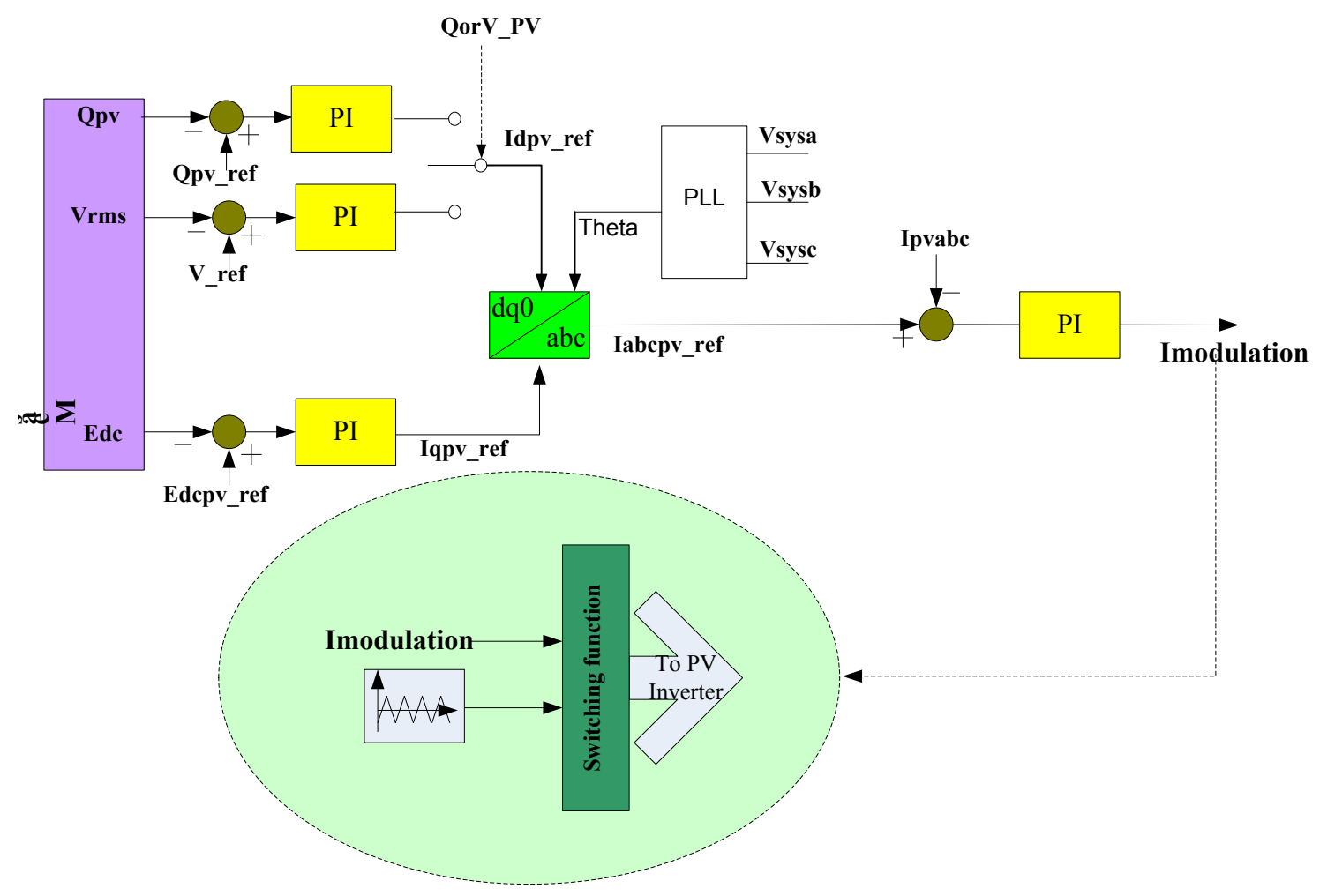

Fig.5.5 PV inverter controller

The controller of battery inverter is similar as PV inverter controller as shown in Fig.5.6. 


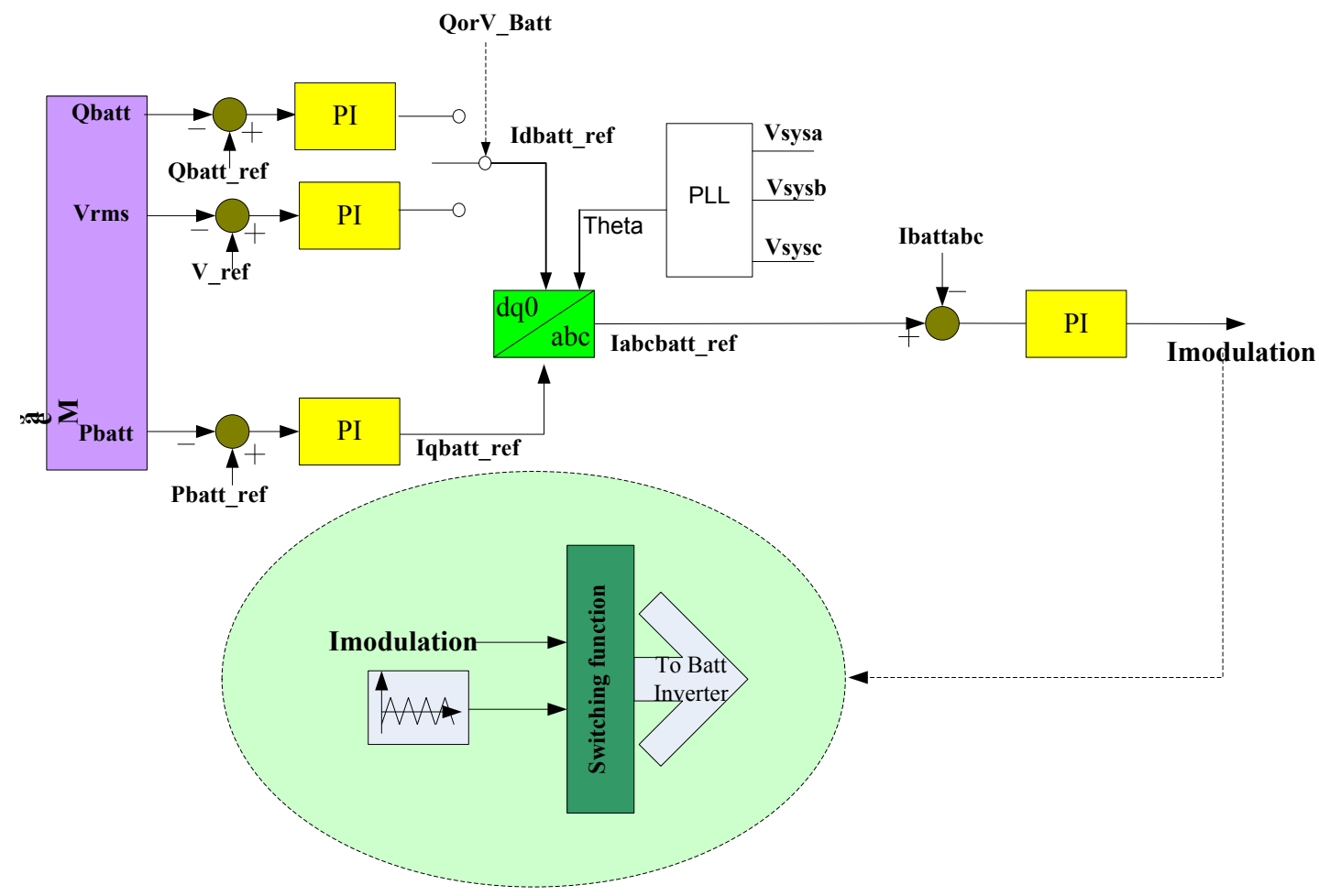

Fig.5.6 Battery inverter controller

\subsection{Reactive Power Coordinated Control Scheme}

PV and Battery inverters can be coordinated to provide dynamic reactive power support. In contrast to the PV panel, battery storage has only limited charging and discharging times so it is not desired to provide reactive power compensation in normal operation. Moreover, oscillations may occur when PV and battery both work in voltage control mode. However the battery should compensate for reactive power when the PCC voltage violatea the limit and the PV inverter is at maximum reactive power output. A coordinated control scheme is designed considering the above mentioned criterions as shown in the flow chart in Fig.5.7 to control the PV and Battery inverters, and restore the hybrid system from over voltage and under voltage problems. The monitored signals in Fig.5.7 are the system parameters that include the reactive power and breaker status of PV, battery and load as well as the PCC bus voltage. Six different operation modes are implemented as follows: 


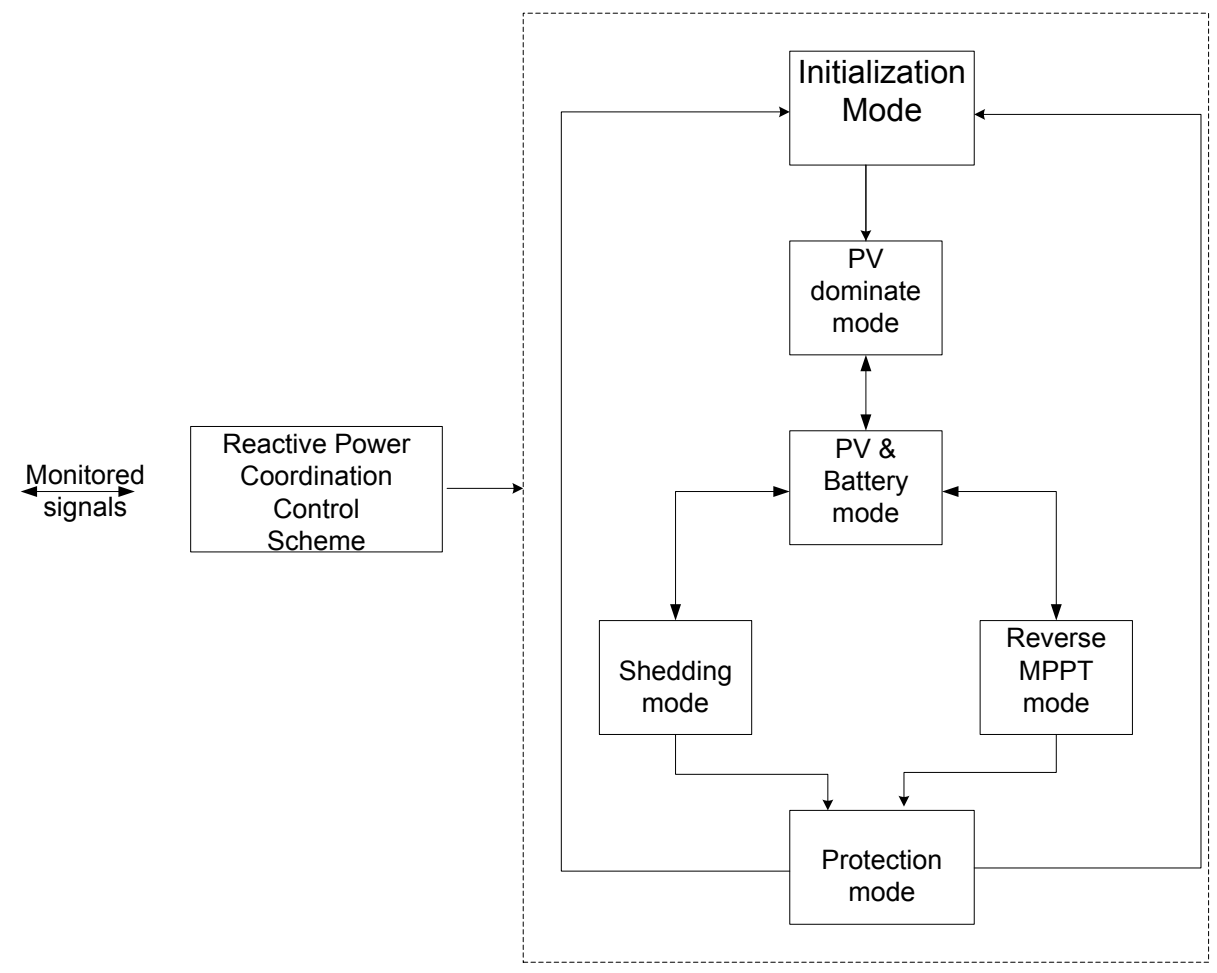

Fig.5.7 The flow chart of reactive power coordination control scheme

1) Initialization mode: This mode occurs during the system starting procedures. The PV starts in 0-RPC mode and then switches to voltage control mode in steady state. The battery starts $2 \mathrm{~s}$ after PV in 0 -RPC mode to reduce transients. PV generates reactive power until PCC bus voltage is constant.

2) PV dominant mode: This mode is activated after the initialization mode where PV is the primary reactive power compensator. In this mode the battery is at zero reactive power output regardless of charging or discharging. In the PV dominant mode small transient disturbances are mitigated by the PV thereby improving the life cycle of battery storage.

3) PV and Battery mode: This mode is activated when the PCC voltage violates the high limit and the PV inverter reaches its maximum reactive power capability. The battery switches to voltage control mode to provide dynamic reactive power support.

4) Shedding mode: This mode is activated when the PCC voltage violates the low limit and both PV and battery are at their maximum reactive power output. Under this condition some of the load may be shed for voltage recovery.

5) Reverse Maximum Power mode: This mode is activated when the PCC voltage violates the high limit and both PV and Battery inverters absorb maximum reactive power. 
The active power output of the PV panels is reduced to increase the PV maximum reactive power capability.

6) Protection Mode: This mode is activated when the PCC voltage violates the protection limits. In order to protect the power electronics device from over voltage or under voltage conditions and other safety issues, both the PV and battery system are disconnected from the power grid.

Table 5.1 gives the control strategy map for each operation mode. With this algorithm, the autonomous operation of PV and battery is achieved, providing flexibility of operation.

TABLE 5.1

CONTROL STRATEGY MAP FOR OPERATION MODES UNDER REACTIVE POWER COORDINATED CONTROL

\begin{tabular}{|c|c|c|c|c|c|c|}
\hline $\begin{array}{c}\text { Operation } \\
\text { mode }\end{array}$ & $\begin{array}{c}\text { PV } \\
\text { booster }\end{array}$ & $\begin{array}{c}\text { PV } \\
\text { inverter }\end{array}$ & $\begin{array}{l}\text { Battery } \\
\text { Inverter }\end{array}$ & $\begin{array}{c}\text { PV } \\
\text { breaker }\end{array}$ & $\begin{array}{l}\text { Battery } \\
\text { breaker }\end{array}$ & $\begin{array}{c}\text { Dynamic } \\
\text { Load breaker }\end{array}$ \\
\hline $\begin{array}{c}\text { Starting } \\
\text { mode }\end{array}$ & MPPT & 0-RPC & 0-RPC & Close & Close & Close \\
\hline $\begin{array}{l}\text { PV dominate } \\
\text { mode }\end{array}$ & MPPT & Voltage control & 0-RPC & Close & Close & Close \\
\hline $\begin{array}{c}\text { PV and Battery } \\
\text { mode }\end{array}$ & MPPT & Voltage control & Voltage control & Close & Close & Close \\
\hline $\begin{array}{c}\text { Shedding } \\
\text { mode }\end{array}$ & MPPT & Voltage control & Voltage control & Close & Close & Open \\
\hline $\begin{array}{l}\text { RMPPT } \\
\text { mode }\end{array}$ & RMPPT & Voltage control & Voltage control & Close & Close & Close \\
\hline $\begin{array}{c}\text { Protection } \\
\text { Mode }\end{array}$ & MPPT & 0-RPC & 0-RPC & Open & Open & Open \\
\hline
\end{tabular}

The proposed control scheme is implemented in RTDS, and the flow chart of the reactive power coordination process is shown in Fig.5.8, 


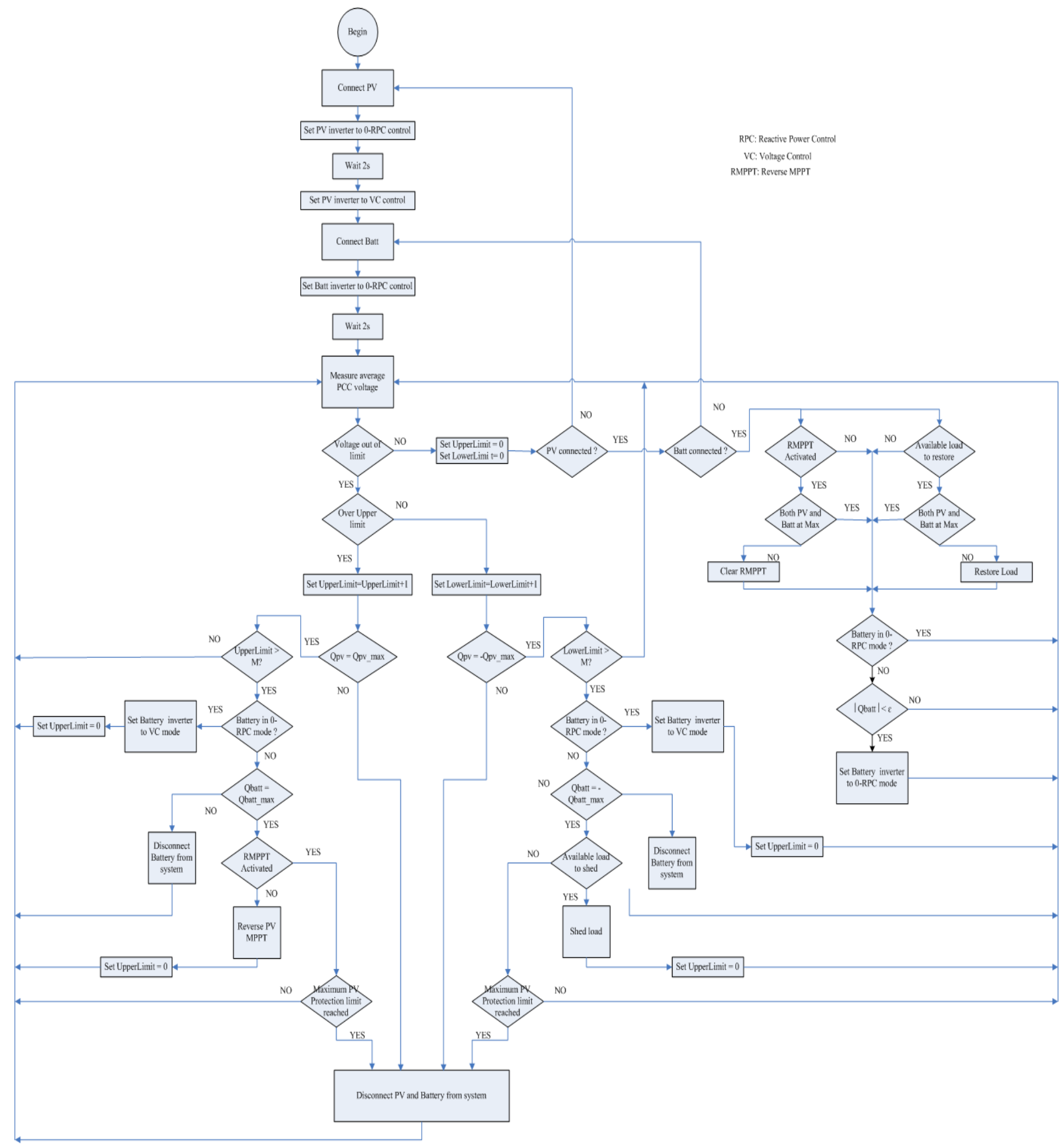

Fig.5.8 Flow chart of reactive power coordination for RTDS implementation

\subsection{Active Power Coordinated Control Scheme}


The uncontrollable nature of solar energy leads to intermittent active power supply and hence unbalance between supply and demand which can be mitigated by battery energy as shown in Eq. (5.10)

$$
P_{P V}+P_{B E S S}=P_{\text {Load }}
$$

where $P_{P V}$ is the power generated by PV plant, $P_{B E S S}$ is the power generated by battery set, and $P_{\text {Load }}$ is the demand. If $P_{P V}$ is larger than demand, the battery is charged, otherwise, it is discharged.

\subsection{Battery operation control}

The battery balances the power supply and demand as well as provides dynamic reactive power support, and hence the direction of power flow through battery frequently changes. In order to increase the life span of battery storage without compromising the control objectives, a charging and discharging control scheme is developed. The battery operation control is shown in (5.11),

$$
\begin{aligned}
& \qquad \text { If } S O C>S O C \_l o w \text { and Pbattset }>0 \text {, discharging mode } \\
& \text { If } S O C<S O C \_ \text {up and Pbattset<0, charging mode }
\end{aligned}
$$

where, SOC_low and SOC_up are the lower and upper limits of SOC, Pbattset is the reference active power output of battery. A hysteresis band and an idle mode are added in addition to the charging and discharging modes. In the idle mode, battery is connected to the system but doesn't participate in the control scheme. Considering the direction of battery to grid as positive, the battery will switch to idle mode if one of the criterions is met-

1) The reference power is within the deadband [-Pband, + Pband].

2) The reference power is higher than the upper limit of deadband, and the SOC of battery is lower than SOC_low.

3) The reference power is lower than the lower limit of deadband, and the SOC of battery is higher than SOC_up. However, once activated, charging won't be deactivated until SOC reach 100 percent unless discharging mode is needed.

\subsection{Optimal PI Controller Tuning Using Genetic Algorithm}


It is seen from 5.3 and 5.4 that a large number of PI control blocks are used in the PV and battery inverters controllers, and hence a set of PI parameters are essential to obtain the optimal performance of PV and battery system. The analytical approach is discussed and genetic algorithm for optimal PI parameters is proposed.

\subsubsection{Small signal model of PV inverter}

Fig.5.9 shows a typical grid connected PV DC-AC inverter,

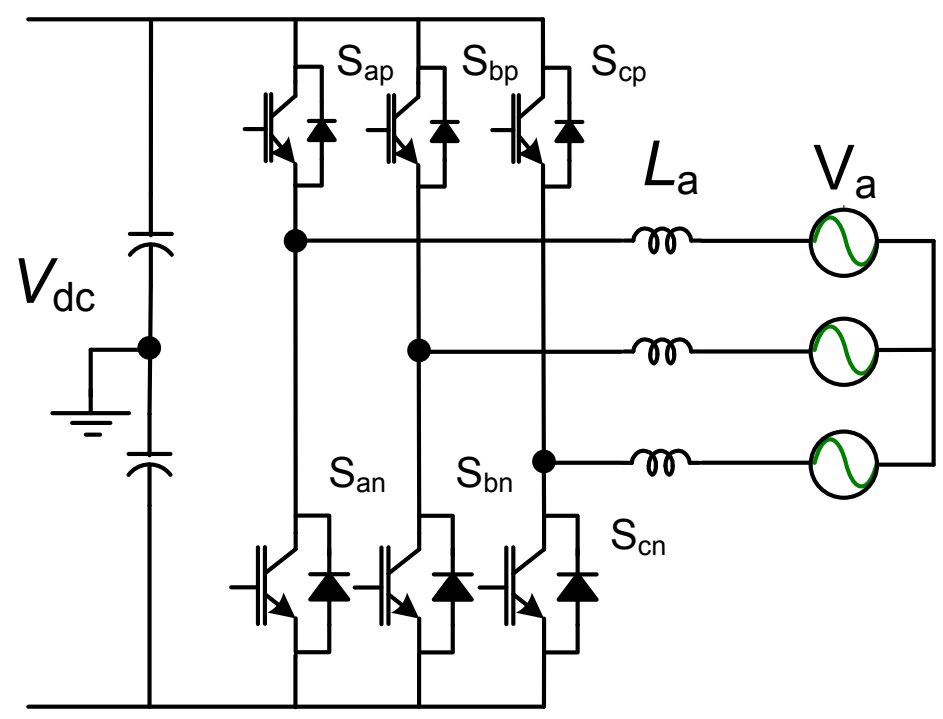

Fig.5.9 Circuit diagram of a PV DC-AC inverter

The specifications of the PV inverter are shown in table 5.2,

TABLE 5.2:

PV INVERTER SPECIFICATIONS

\begin{tabular}{|l|l|}
\hline Parameter & Value \\
\hline$V d c$ & $1000 \mathrm{~V}$ \\
\hline$V a$ & $480 \mathrm{~V}$ (line-to-line) \\
\hline$L$ & $300 \mathrm{uH}$ \\
\hline$f S$ & $6 \mathrm{kHz}$ \\
\hline$C$ & $10000 \mathrm{uH}$ \\
\hline$S$ & $0.8 \mathrm{MVA}$ \\
\hline
\end{tabular}

The large signal average model of the PV inverter developed is shown in Fig.5.10, 

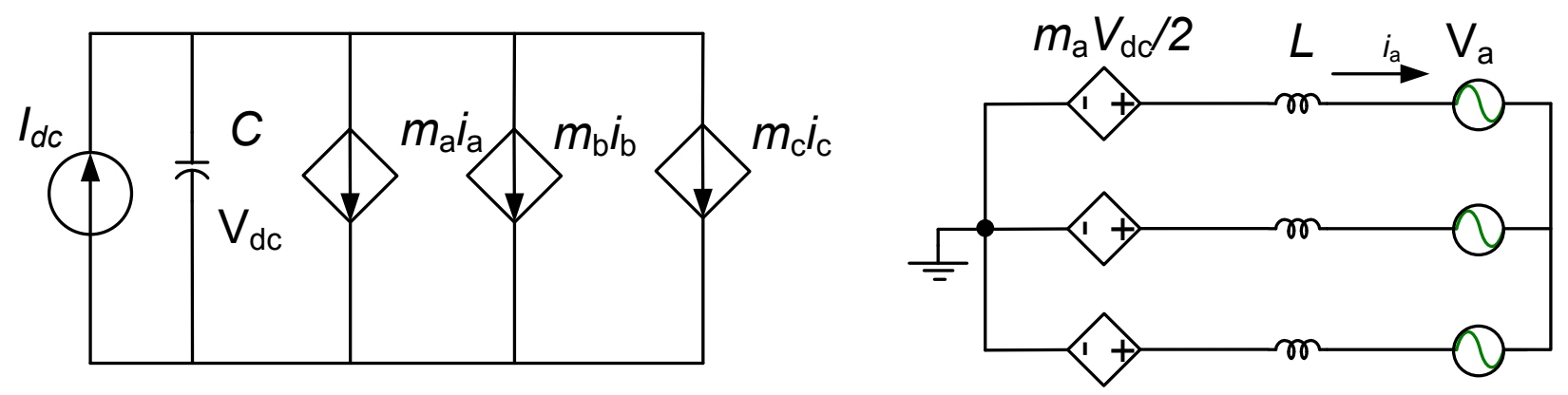

Fig.5.10 Average PV inverter model

The dynamics characteristics of the ac side system is given as,

$$
\left\{\begin{array}{l}
m_{a} \frac{v_{d c}}{2}=L \frac{d i_{a}}{d t}+v_{a} \\
m_{b} \frac{v_{d c}}{2}=L \frac{d i_{b}}{d t}+v_{b} \\
m_{c} \frac{v_{d c}}{2}=L \frac{d i_{c}}{d t}+v_{c}
\end{array}\right.
$$

Similarly, for dc side, we can get,

$$
C \frac{d v_{d c}}{d t}=i_{d c}-\left(m_{a} i_{a}+m_{b} i_{b}+m_{c} i_{c}\right)
$$

Applying the transformation matrix shown in equation (5.14),

$$
T=\sqrt{\frac{2}{3}}\left[\begin{array}{ccc}
\cos (\omega t) & \cos (\omega t-120) & \cos (\omega t-240) \\
\sin (\omega t) & \sin (\omega t-120) & \sin (\omega t-240) \\
\sqrt{\frac{1}{2}} & \sqrt{\frac{1}{2}} & \sqrt{\frac{1}{2}}
\end{array}\right]
$$

The state equations in $d q$-frame can be obtained as shown in equation (5.15),

$$
\left\{\begin{array}{l}
L \frac{d i_{q}}{d t}=m_{q} \frac{v_{d c}}{2}-\omega L i_{d}-v_{q} \\
L \frac{d i_{d}}{d t}=m_{d} \frac{v_{d c}}{2}+\omega L i_{q}-v_{d} \\
L \frac{d i_{0}}{d t}=m_{0} \frac{v_{d c}}{2}-v_{0} \\
C \frac{d v_{d c}}{d t}=i_{d c}-\left(m_{q} i_{q}+m_{d} i_{d}+m_{0} i_{0}\right)
\end{array}\right.
$$


Since the zero-sequence components are zero for a balanced three-phase system, the state equations can be reduced to,

$$
\left\{\begin{array}{l}
L \frac{d i_{q}}{d t}=m_{q} \frac{v_{d c}}{2}-\omega L i_{d}-v_{q} \\
L \frac{d i_{d}}{d t}=m_{d} \frac{v_{d c}}{2}+\omega L i_{q}-v_{d} \\
C \frac{d v_{d c}}{d t}=i_{d c}-\left(m_{q} i_{q}+m_{d} i_{d}\right)
\end{array}\right.
$$

If we choose $i_{q}, i_{d}$ and $v_{d c}$ as state variables $x, m_{q}$ and $m_{d}$ as control inputs $u$, and $v_{q}, v_{d}$ and $i_{d c}$ as disturbance inputs $v$, the state equation can be expressed as,

$$
\frac{d x}{d t}=f(x, u, v)
$$

Here,

$$
x=\left[\begin{array}{c}
i_{q} \\
i_{d} \\
v_{d c}
\end{array}\right], u=\left[\begin{array}{l}
m_{q} \\
m_{d}
\end{array}\right], f(x, u, v)=\left[\begin{array}{c}
m_{q} \frac{v_{d c}}{2 L}-\omega i_{d}-\frac{v_{q}}{L} \\
m_{d} \frac{v_{d c}}{2 L}+\omega i_{q}-\frac{v_{d}}{L} \\
\frac{i_{d c}}{C}-\frac{1}{C}\left(m_{q} i_{q}+m_{d} i_{d}\right)
\end{array}\right]
$$

The state equations can be linearized around an equilibrium point $(X, U, V)$ where $(\tilde{x}, \tilde{u}, \tilde{v})$ is the perturbation vector around the operation point, then the state variables and the state model can be expressed as (5.18) and (5.19), respectively,

$$
\begin{aligned}
& \left\{\begin{array}{l}
x=X+\tilde{x} \\
u=U+\tilde{u} \\
v=V+\tilde{v}
\end{array}\right. \\
& \frac{d x}{d t}=f(X, U, V)+\frac{\partial f(x, u, v)}{\partial x}\left|(X, U, V) \widetilde{x}+\frac{\partial f(x, u, v)}{\partial u}\right|(X, U, V) \widetilde{u}+\frac{\partial f(x, u, v)}{\partial v} \mid(X, U, V) \widetilde{v}
\end{aligned}
$$

The linearized small-signal state equations are given as, 


$$
\left[\begin{array}{c}
\frac{d \widetilde{i}_{q}}{d t} \\
\frac{d \widetilde{i_{d}}}{d t} \\
\frac{d \widetilde{v}_{d c}}{d t}
\end{array}\right]=\left[\begin{array}{ccc}
0 & -\omega & \frac{M_{q}}{2 L} \\
\omega & 0 & \frac{M_{d}}{2 L} \\
\frac{-M_{q}}{C} & \frac{-M_{d}}{C} & 0
\end{array}\right]\left[\begin{array}{c}
\widetilde{i}_{q} \\
\widetilde{i}_{d} \\
\widetilde{v}_{d c}
\end{array}\right]+\left[\begin{array}{cc}
\frac{V_{d c}}{2 L} & 0 \\
0 & \frac{V_{d c}}{2 L} \\
\frac{-I_{q}}{C} & \frac{-I_{d}}{C}
\end{array}\right]\left[\begin{array}{c}
\widetilde{m}_{q} \\
\widetilde{m}_{d}
\end{array}\right]+\left[\begin{array}{ccc}
-\frac{1}{L} & 0 & 0 \\
0 & -\frac{1}{L} & 0 \\
0 & 0 & \frac{1}{C}
\end{array}\right]\left[\begin{array}{c}
\widetilde{v}_{q} \\
\widetilde{v}_{d} \\
\widetilde{i}_{d c}
\end{array}\right]
$$

The steady-state operation point can be found as,

$$
\left\{\begin{array}{l}
M_{q} \frac{V_{d c}}{2}=V_{q}+\omega L I_{d} \\
M_{d} \frac{V_{d c}}{2}=V_{d}-\omega L I_{q} \\
I_{d c}=\left(M_{q} I_{q}+M_{d} I_{d}\right)
\end{array}\right.
$$

The passive power generated by the inverter can be expressed mathematically as

$$
Q=v_{q} i_{d}-v_{d} i_{q}
$$

Usually, we find an appropriate initial phase angle that to make $v_{d}$ as zero. Therefore the passive power can be simplified to be,

$$
Q=v_{q} i_{d}
$$

Thus, the passive power only depends on the $d$-axis current. In the case of a grid-connected inverter, the grid voltage can be assumed to be constant; therefore its perturbation should be zero,

$$
\left[\begin{array}{l}
\widetilde{v}_{q} \\
\widetilde{v}_{d}
\end{array}\right]=\left[\begin{array}{l}
0 \\
0
\end{array}\right]
$$

\subsubsection{Analytical solution}

The control objectives of grid connected inverter of PV are to keep constant dc-link voltage and deliver required reactive power. The control block is shown in Fig.5.11. 


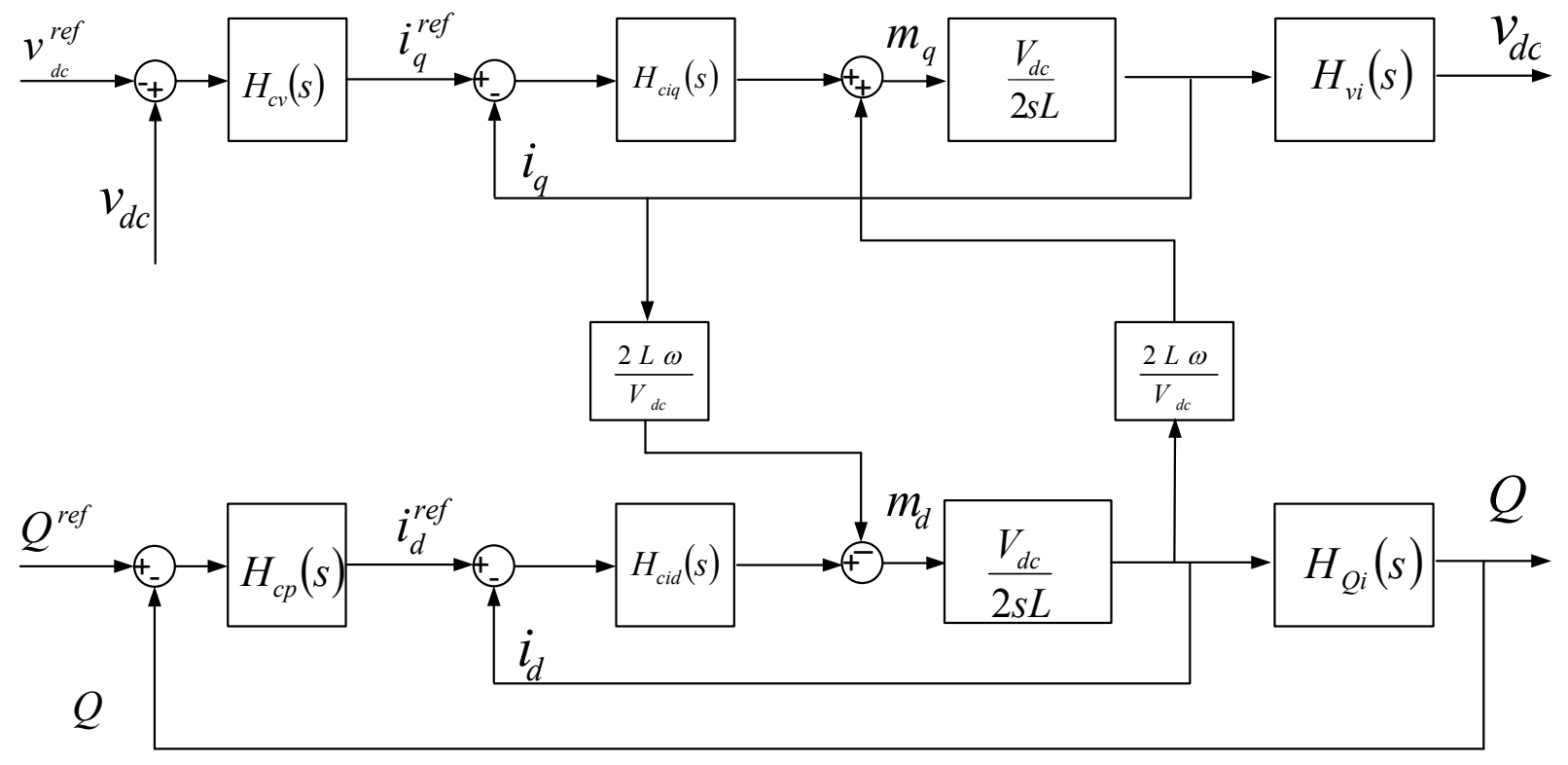

Fig.5.11 Control block of a grid connected inverter for PV system

The dual-loops are utilized to form the control system. The inner loop is to regulate the active and reactive power currents, respectively, and the outer loops are employed to track the desired dc-link voltage and reactive power. Since in a well designed dual loop control system the response of the inner loop is much faster than that of the outer loop, it can be assumed that the state variables of the outer loop are constant for the inner loop. Therefore the state equations of the inner loop can be simplified to be,

$$
\left[\begin{array}{c}
\frac{d \widetilde{i_{q}}}{d t} \\
\frac{d \widetilde{i_{d}}}{d t}
\end{array}\right]=\left[\begin{array}{cc}
0 & -\omega \\
\omega & 0
\end{array}\right]\left[\begin{array}{c}
\tilde{i}_{q} \\
\widetilde{i_{d}}
\end{array}\right]+\left[\begin{array}{cc}
\frac{V_{d c}}{2 L} & 0 \\
0 & \frac{V_{d c}}{2 L} \\
\frac{-I_{q}}{C} & \frac{-I_{d}}{C}
\end{array}\right]\left[\begin{array}{l}
\widetilde{m}_{q} \\
\tilde{m}_{d}
\end{array}\right]+\left[\begin{array}{ccc}
-\frac{1}{L} & 0 & 0 \\
0 & -\frac{1}{L} & 0 \\
0 & 0 & \frac{1}{C}
\end{array}\right]\left[\begin{array}{c}
\tilde{v}_{q} \\
\widetilde{v}_{d} \\
\tilde{i}_{d c}
\end{array}\right]
$$

It can be observed that the transfer function from the control input (modulation indexes) to outputs (currents) is

$$
H_{i q}(s)=H_{i d}(s)=\frac{V_{d c}}{2 L s}
$$

Since the plant is a first-order system and the pole locates in the origin, the Proportion controller can be used to adjust the bandwidth of the system. The loop gain is 


$$
L_{i}(s)=H_{c i q}(s) H_{i q}(s)=\frac{k_{p} V_{d c}}{2 L s}
$$

The maximum bandwidth is selected as the one fifth of the switching frequency $(10 \mathrm{kHz})$, which is $2 \mathrm{kHz}$. Substituting the parameters and bandwidth into (5.26), $k_{p}$ can be obtained,

$$
k_{p}=\frac{2 L_{s} L_{i}}{V_{d c}}
$$

For the passive power loop, the transfer function is,

$$
H_{Q i}(s)=\frac{\widetilde{q}}{\widetilde{i_{d}}}=V_{q}
$$

The integrator controller is utilized to regulate the outer loop. The loop transfer function after compensation is

$$
L_{q}(s)=H_{c q}(s) H_{q}(s)=\frac{k_{i} V_{q}}{s}
$$

In order to decouple the inner current loop and the outer passive power loop, the bandwidth of the outer loop should be under $(1 / 5 \sim 1 / 10)$ of the bandwidth of the inner current loop. Herein the maximum bandwidth of the outer voltage loop is set to be $240 \mathrm{~Hz}$, the parameter of the integrator controller can be obtained, which is

$$
k_{i}=\frac{L_{q} s}{V_{q}}
$$

From (5.24), the dc-link voltage $\widetilde{v_{d c}}$ can be regulated by $\widetilde{l_{q}}$ and $\widetilde{\imath_{d}}$, but $M_{q}$ is much larger than $M_{d}$; therefore the term of $\widetilde{\imath_{q}}$ is dominant. It is reasonable that only $\widetilde{\imath_{q}}$ is regulated to control the dc-link voltage, and the transfer function can be expressed mathematically as

$$
H_{v i}(s)=-\frac{M_{q}}{C s}
$$

An integrator controller can be used, and the loop transfer function is

$$
L_{v}(s)=H_{c v}(s) H_{v}(s)=-\frac{k_{p} M_{q}}{C s}
$$


The $M_{q}$ can be obtained around an operation point, which is about 0.64 . The maximum bandwidth of the outer voltage loop is also $240 \mathrm{~Hz}$. The parameter of the controller is obtained by,

$$
k_{p}=\frac{L_{v} C s}{M_{q}}
$$

\subsubsection{Genetic algorithm implementation}

As mentioned before, the genetic algorithm consists of three steps: initialization, evaluation and reproduction. The optimization scheme used in this work is as shown in Fig.5.12,

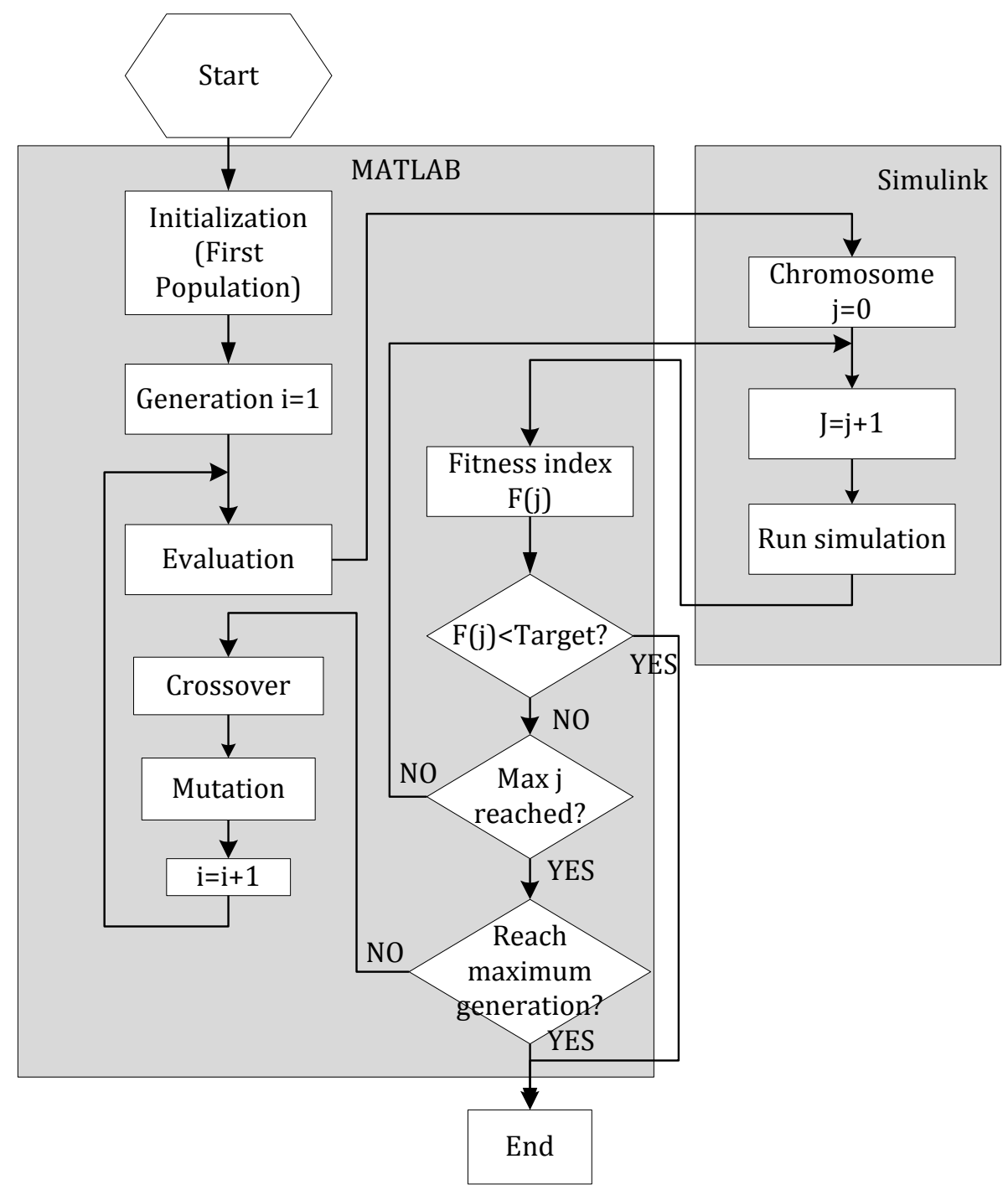

Fig.5.12 Optimization procedures using GA 
It is seen that the first generation is initialized in MATLAB, and the control parameters included in each chromosome is decoded and sent to the PI controllers of the SIMULINK model one by one. The simulation results are sent back to MATLAB for evaluation and reproduction. The optimum PI parameters are obtained when a fitness index reaches desired value or certain generation is met. The detail implementation is discussed in below.

\section{1) Initialization}

From the analysis in 5.6.1 and 5.6.2, it is known that the proportion and integrator controller are designed to regulate the real and reactive power output of PV inverter, respectively. Then for a system with a single PV inverter, four parameters are needed to be optimized by the genetic algorithm. Accordingly, the number of genes in each chromosome is four, and the range of the control parameter is within [0 10]. The chromosome structure is as shown in Fig.5.13,

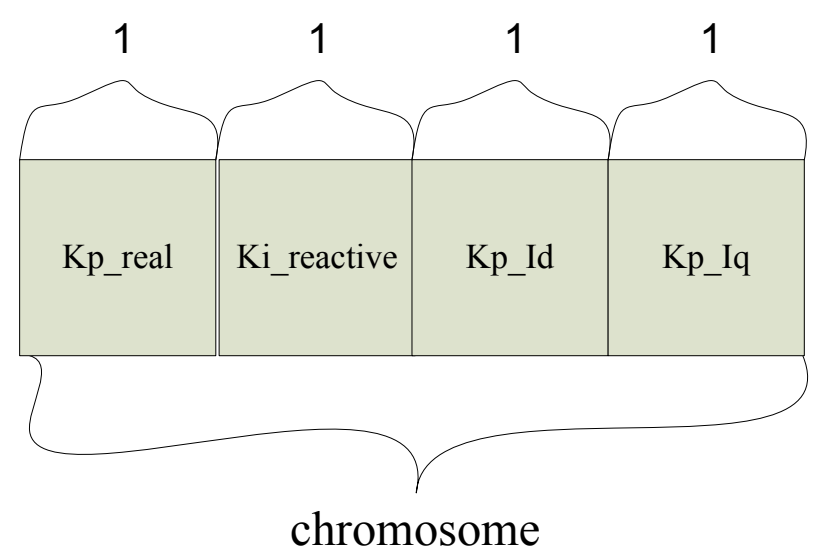

Fig.5.13 The structure of the chromosome

The population size is set as 500 and the chromosomes of the initial population are then generated by random pick from [0 10].

2) Evaluation

A grid connected PV model is modeled in SIMULINK as shown in Fig.5.14 


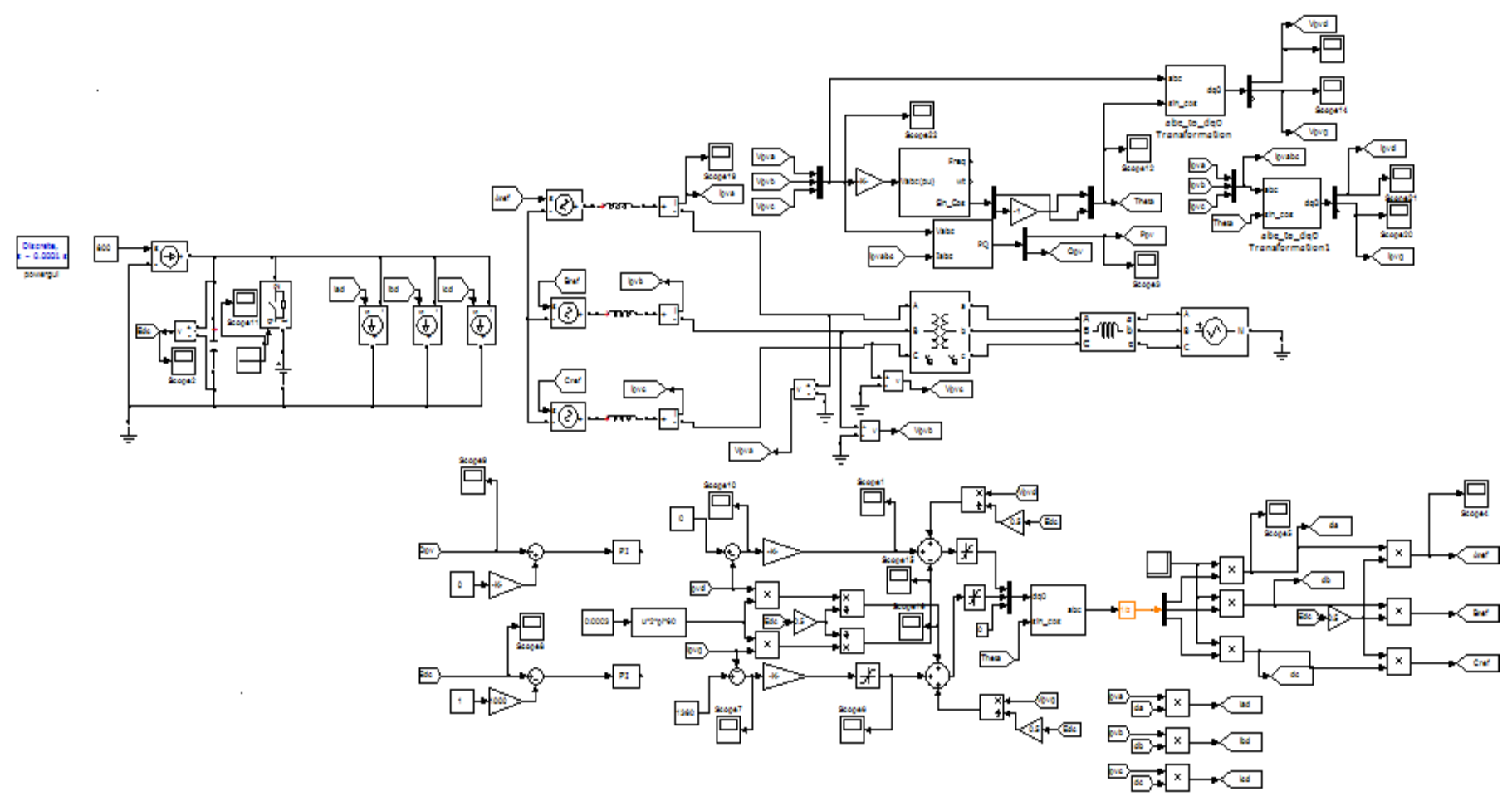

Fig.5.14 Screenshot of the Simulink model of a grid connected PV system

The control parameters of the real and reactive power loops, $d$-axis and $q$-axis current loop are defined as Kp_real and Ki_reactive, $K p_{-} I d$ and $K p_{-} I q$, respectively. The errors between reference and actual value for the four control variables are sent to genetic algorithm for evaluation.

The genetic algorithm proceeds with selection and reproduction, and the optimized result is the decoded solution when termination condition has been reached. A sufficiently small time step $T_{S}$ ensures the errors as constant during each time step. The simulation result contains the information of the errors between reference and actual value for the four control variables, and is evaluated by a pre-defined fitness function. The fitness of the chromosomes is described as the reciprocal of the summation of the absolute integration of the error and a summation of the absolute integration of the error is used as reference for calculating fitness index of each chromosome as show in Fig.5.15. All the chromosomes are then evaluated and ranked by the fitness index. 


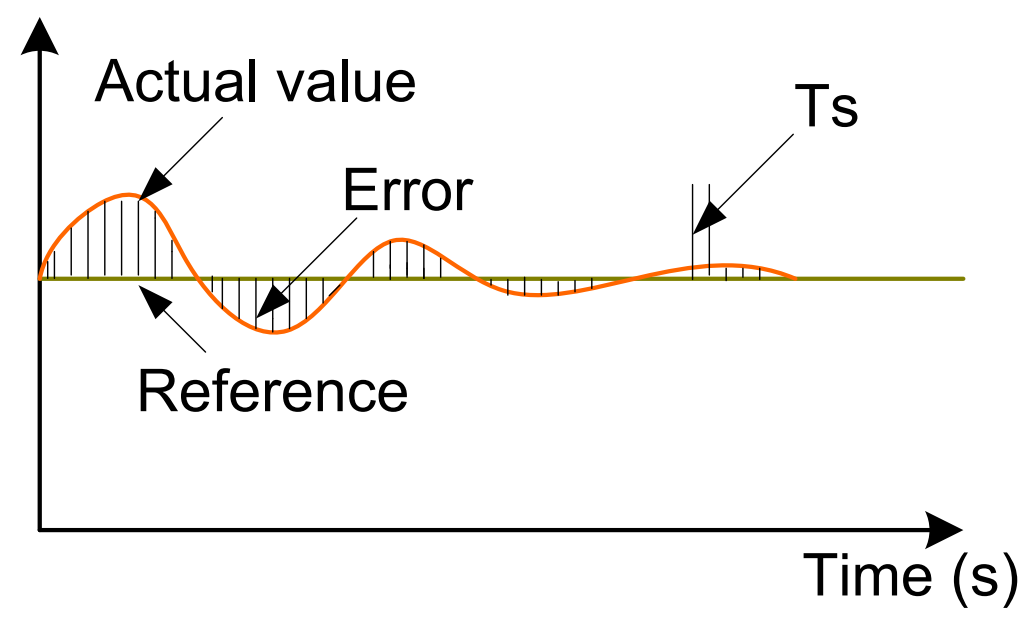

Fig.5.15 Fitness evaluation diagram

\section{3) Reproduction}

To ensure the chromosome that has the higher index has better chance to be selected as the parent for reproduction, Roulette Wheel Selection Algorithm as shown in Fig.5.16 is applied in this work to choose the parent chromosomes.

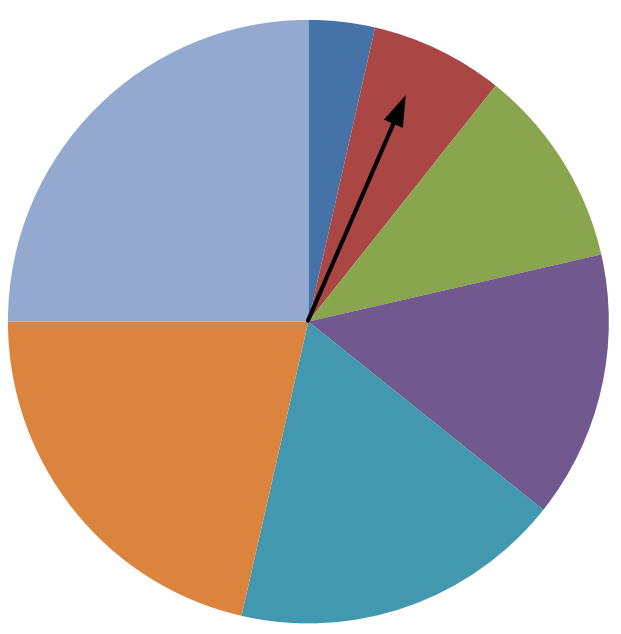

Fig.5.16 Roulette Wheel Selection Algorithm

Single point crossover with $80 \%$ crossover rate is applied combine with the top $20 \%$ ranked chromosomes from the last generation to form the next generation. A mutation with $10 \%$ mutation rate is then applied to the new generation to restore any lost information. This generational process is repeated until the highest ranking solution's fitness is reaching or has 
reached a plateau such that successive iterations no longer produce better results. The data used in Table 5.2 is used and the GA result gives similar result as hand calculation.

\subsection{Simulation Result}

The proposed system as shown in Fig.5.1 is modeled in RSCAD to verify the proposed control strategy. The rating of PV and battery is $0.7 \mathrm{MW}$ and $0.3 \mathrm{MW}$ respectively. The rated system voltage is $11 \mathrm{kV}$ on the primary side and $0.48 \mathrm{kV}$ on the secondary side. Several scenarios are simulated and the results are presented in this section.

\subsubsection{Initialization progress}

A constant insolation of $1000 \mathrm{~W} / \mathrm{m}^{2}$ is given as input to the $\mathrm{PV}$ array which is interconnected at 0s. The total load of the system is 0.6MW. The initialization progress is as shown in Fig.5.17, where, Ppvac and Qpvac are the PV inverter's active and reactive power outputs, Pbattac, Qbattac are the battery inverter's active and reactive power outputs, $P L$ and $Q L$ are the active and reactive power demands of the fixed impedance load, Pmon and Qmon are the active and reactive power demands of the dynamic load, $P S$ and $Q S$ is the active and reactive power of the grid, and Vpccrms is the RMS value of the PCC bus voltage. It is seen that a total $0.6 \mathrm{MW}$ load is supplied by the grid before PV integration. The PV and battery supply load after the connection of battery storage. Under MPPT control the PV generates 0.7MW active power and $0.1 \mathrm{MW}$ power is used to charge the battery. The reactive power control of the PV farm changes from 0-RPC to voltage control after the connection of PV farm and Qpvac increases from 0 Mvar to 0.36 Mvar while battery reactive power output is maintained at 0 Mvar. Vpccrms is maintained at rated value $0.48 \mathrm{kV}$ under voltage control of the PV farm. Two voltage spikes as seen in Fig.5.17 are due to the operation mode changes and the interconnection of the battery. 

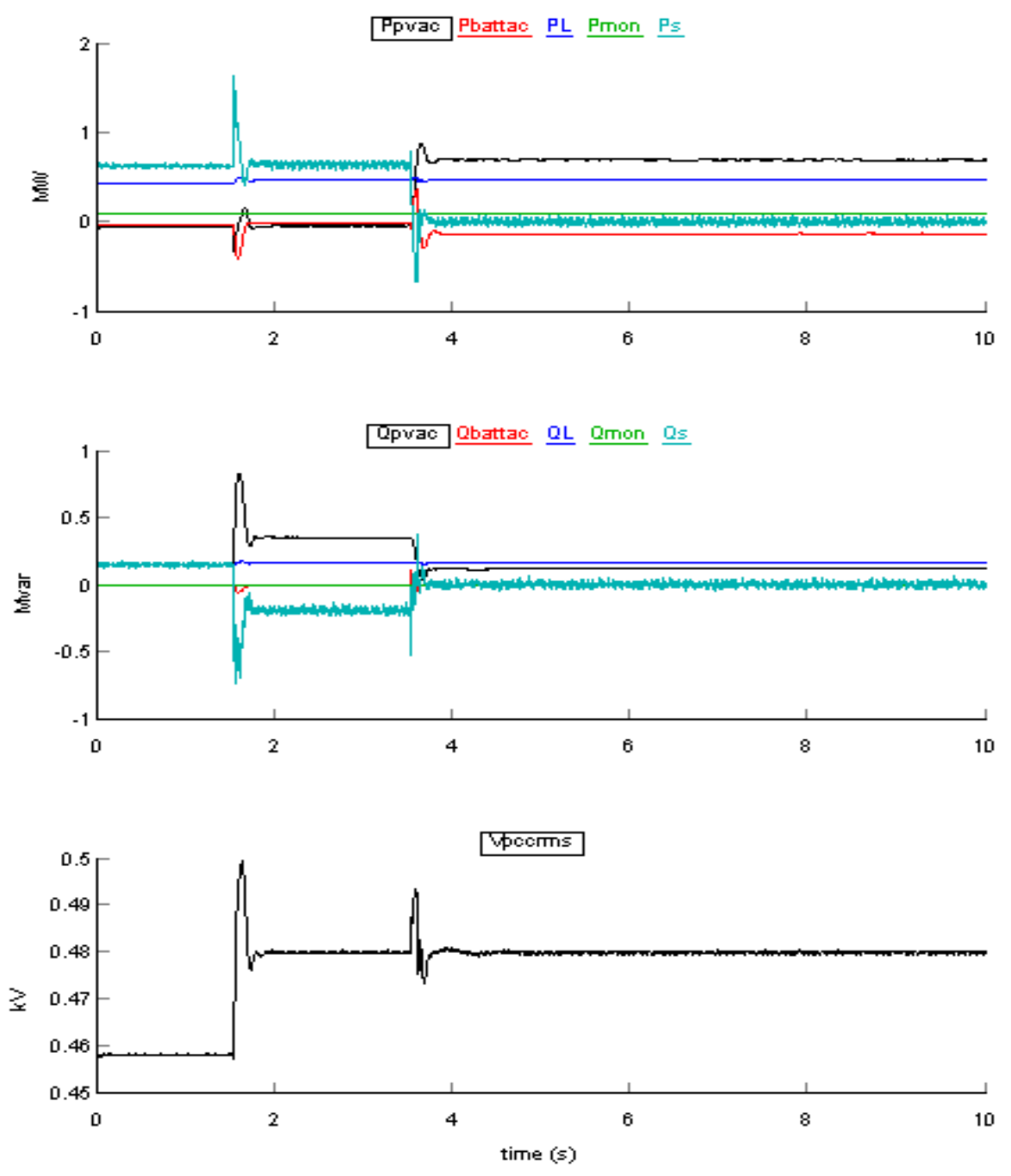

Fig.5.17 Simulation results of the hybrid system under initialization mode

\subsection{MW load step change}

A constant insolation of $800 \mathrm{~W} / \mathrm{m}^{2}$ is given as the input to the $\mathrm{PV}$ array. The total load changes from $0.77 \mathrm{MW}$ to $0.57 \mathrm{MW}$ at 2s. It is seen from Fig.5.18 that the PV farm is at its maximum active power (0.6 MW). The battery is controlled by the active power coordination controller to compensate for the mismatch between PV generation and the load. Battery generates 0.17 MW active power from $0-2 \mathrm{~s}$ and absorbs $0.03 \mathrm{MW}$ active power after $2 \mathrm{~s}$. The hybrid system is under PV dominant mode where PV generates reactive power to maintain the PCC bus voltage constant, and Qpvac shows a variation pattern that is similar to that of the load change. 

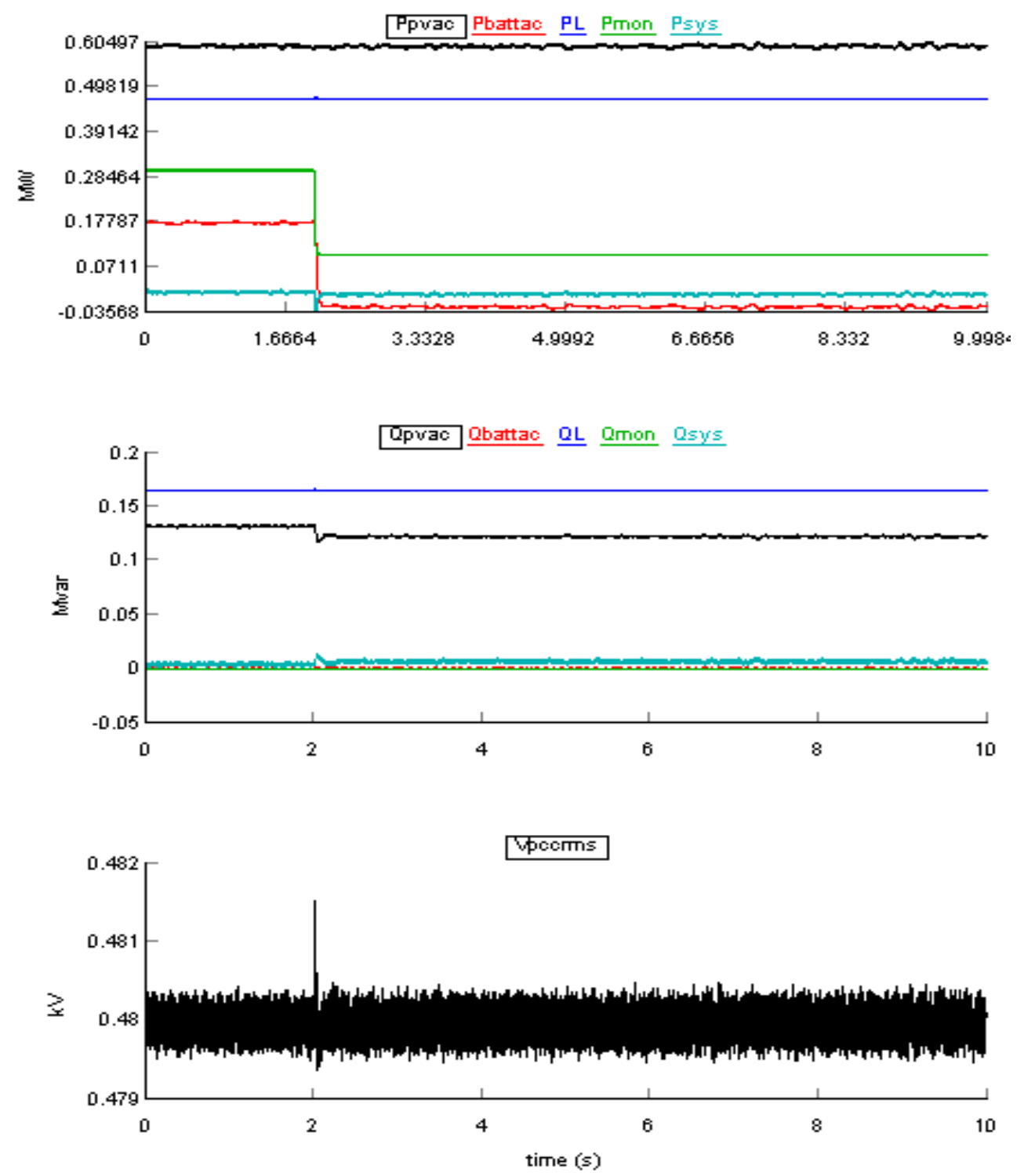

Fig.5.18 Simulation results of the hybrid system under load step change from $0.77 \mathrm{MW}$ to $0.57 \mathrm{MW}$

\subsection{Mvar load step change}

A constant insolation of $1000 \mathrm{~W} / \mathrm{m}^{2}$ is the input to the PV array. The hybrid system is initially working under PV dominate mode, and a 0 to 0.5 MVAR step change is applied to the dynamic load at 2s. The system performance is shown in Fig.5.19, where QorVpv is the PV reactive power control mode (0: 0-RPC, 1:Voltage control), and QorVbatt is the Battery reactive power control mode (0: 0-RPC, 1: Voltage control). Fig.5.19 shows that Vpccrms violates the low voltage limit at 2 s even when Qpvac is reaching maximum reactive power limit at 0.48 Mvar. The Battery is switched from 0-RPC to voltage regulation control at 4.1s and generates 0.38 MVAR of reactive power and then reduces to 0.30 MVAR in steady state. Vpccrms returns to 
its rated value after the hybrid system is working under PV and Battery mode. Ppvac, Pbattac and $P S$ are not affected by the mode change.
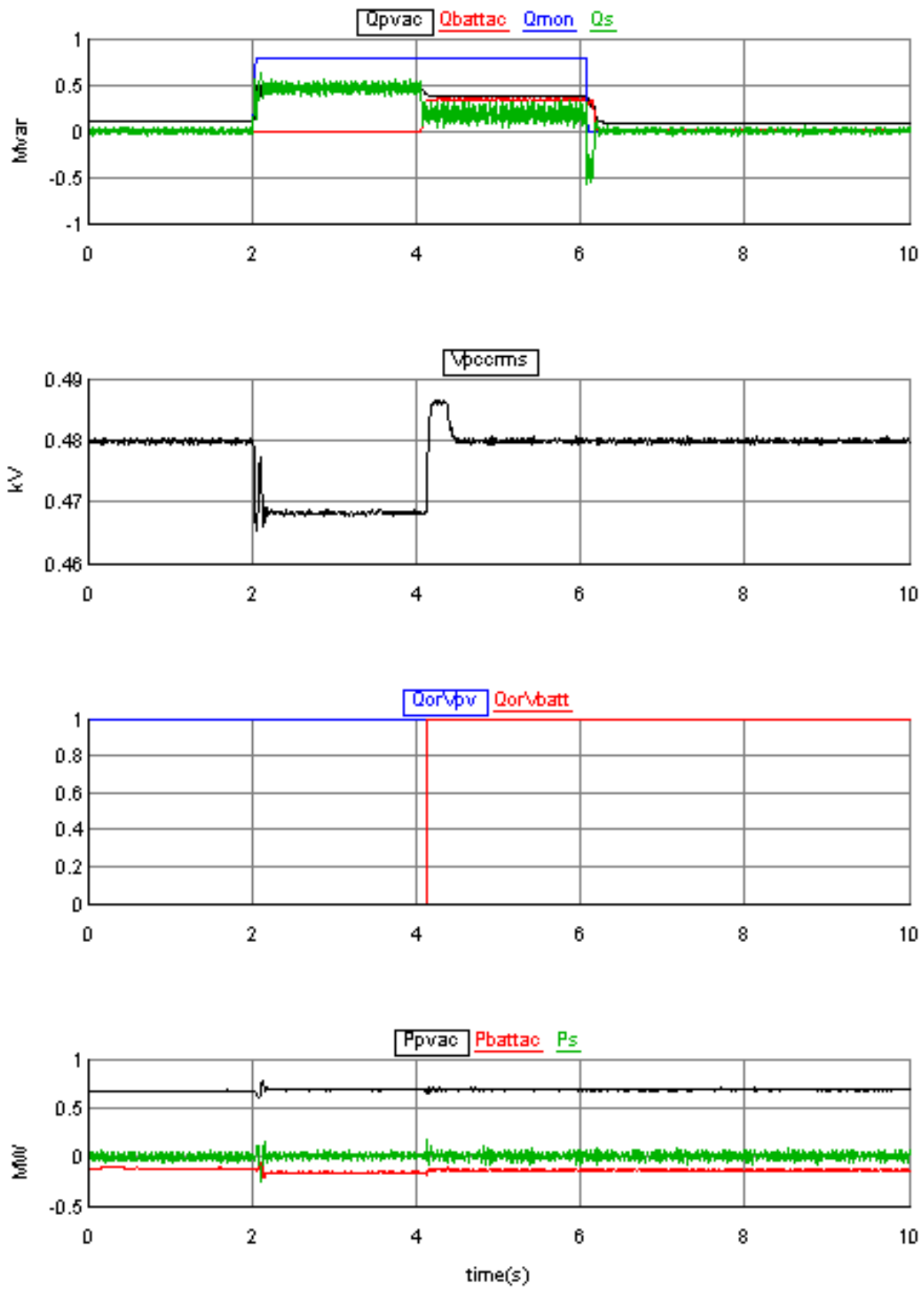

Fig.5.19 Simulation results of the hybrid system under 0.5 Mvar load step change 

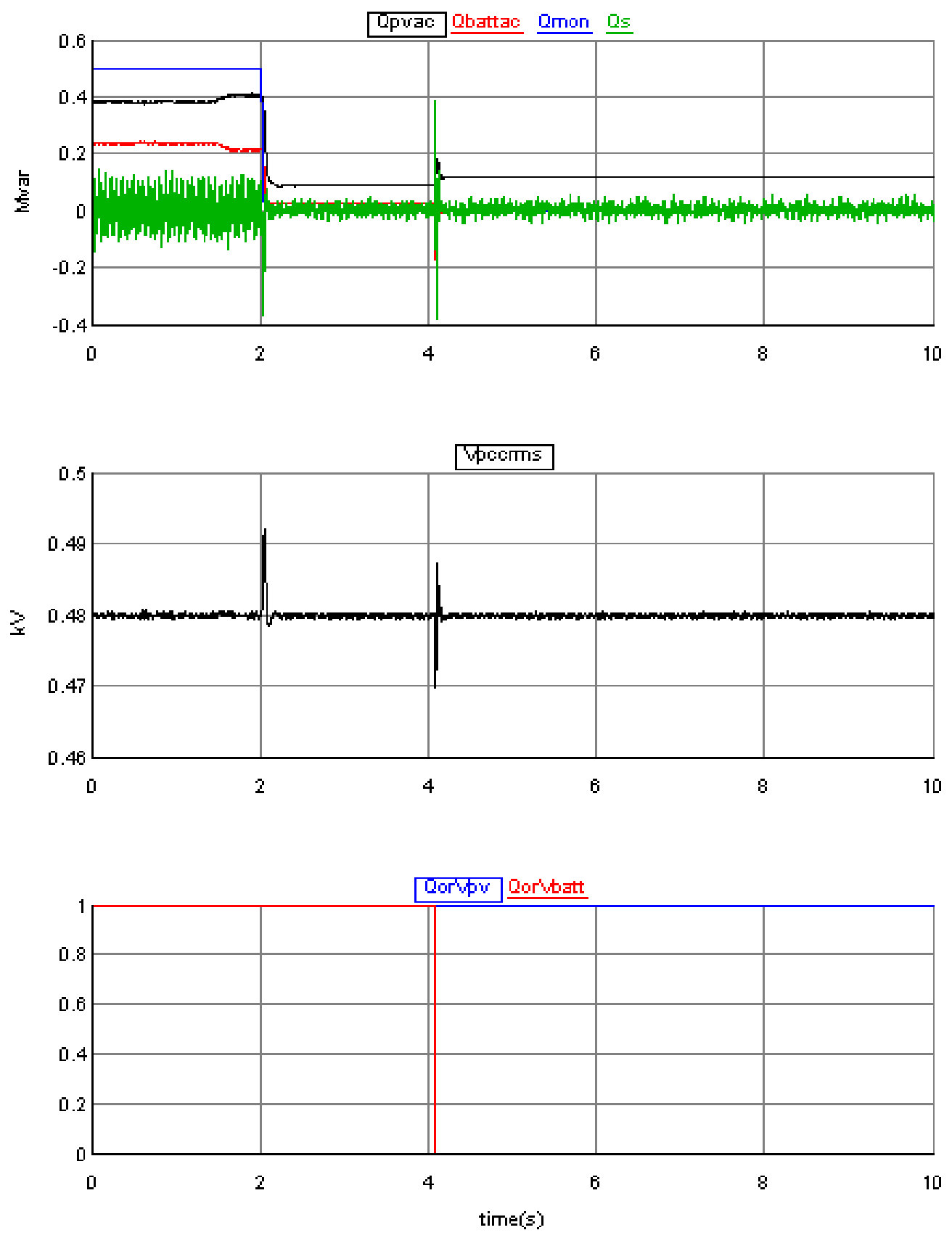

Fig.5.20 Simulation results of the hybrid system when the load changes back from 0.5 Mvar to 0 Mvar

Fig.5.20 shows the system response when the load demand goes back to 0 Mvar at 2s. The reactive power output of PV and battery both reduces at $2 \mathrm{~s}$. The coordinated controller restores the system from PV and battery mode to PV dominate mode at 4s with PV generating reactive power to maintain Vpcrms constant at $0.48 \mathrm{kV}$.

5.7.4 0.8 Mvar load step change 

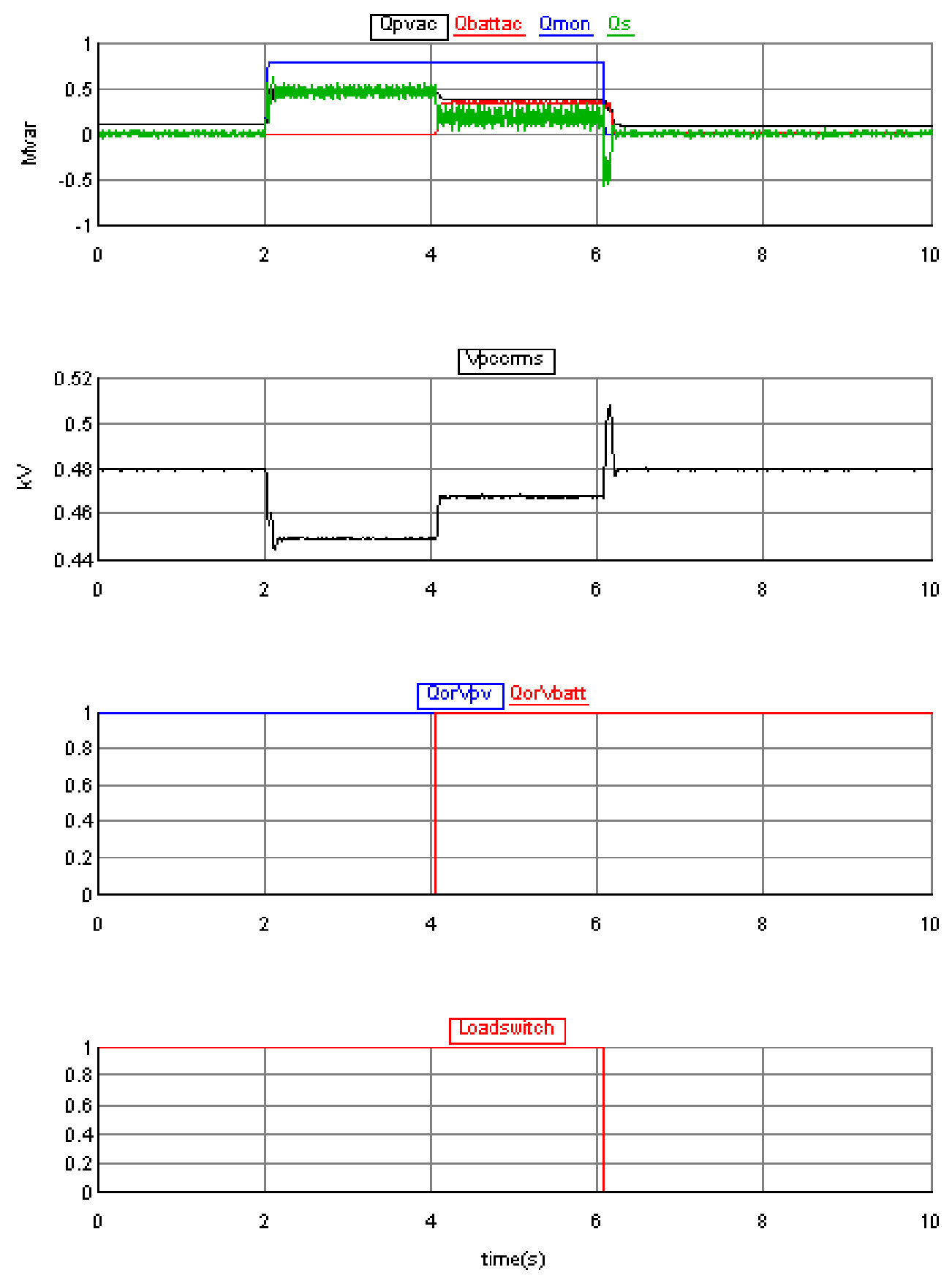

Fig.5.21 Simulation results of the hybrid system under 0.8 Mvar load step change

A constant insolation $800 \mathrm{~W} / \mathrm{m}^{2}$ is given as the input of PV array and the hybrid system is initially working under PV dominate mode. A 0 to 0.8 MVAR step change is applied to the dynamic load at 2s and the system performance is shown in Fig.5.21, where Loadswitch is the status of the dynamic load switch (0: open, 1: close). It is seen that Vpccrms violates the low voltage limit even when PV is generating maximum reactive power at 0.48 Mvar. A voltage 
rise is observed when the system switched to PV and Battery mode, but Vpccrms is still violating the low limit with PV and battery generating maximum reactive power. Shedding mode is activated that disconnects the dynamic load and restores Vpccrms.
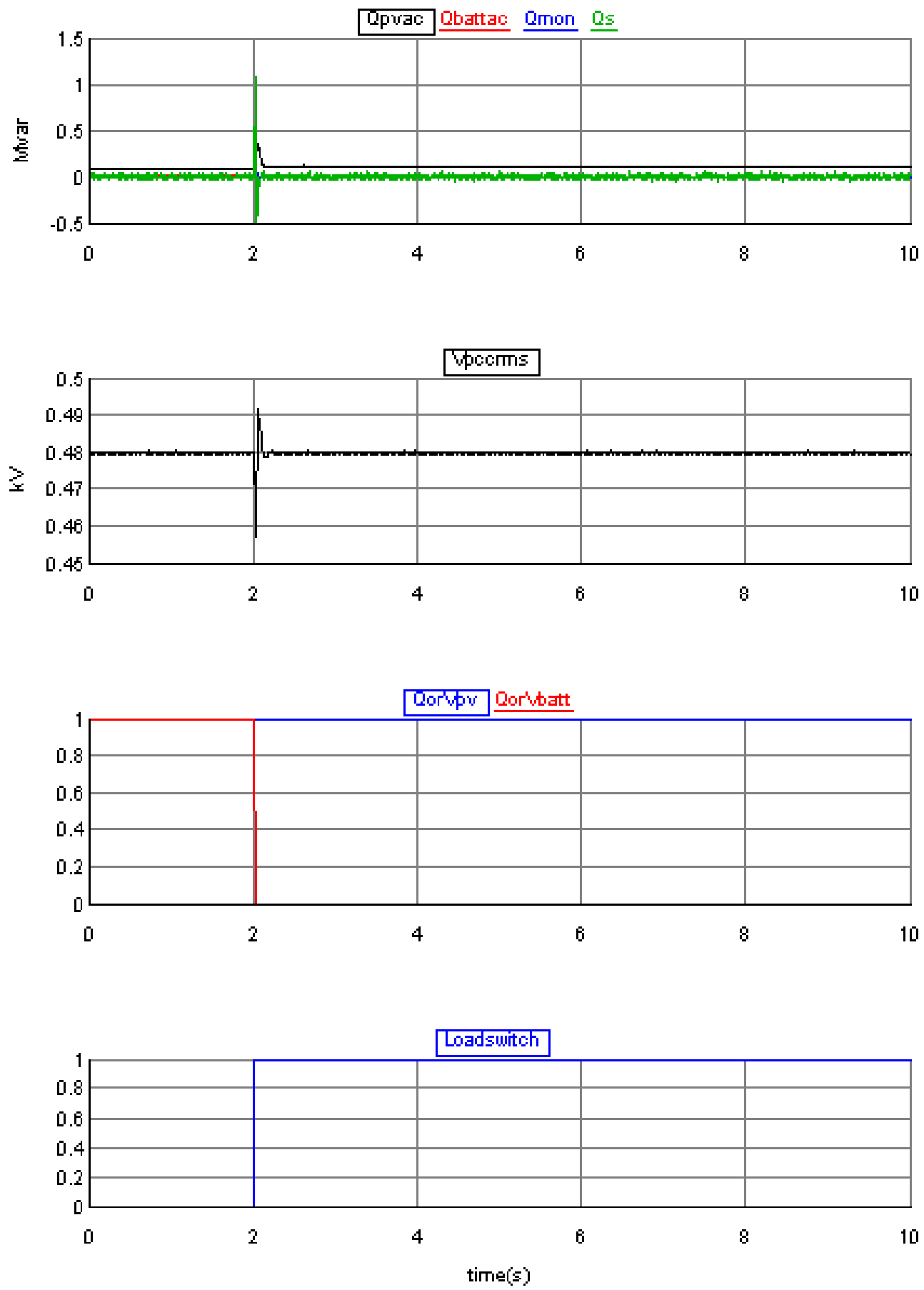

Fig.5.22 Simulation results of the hybrid system when the load changes back from 0.8 Mvar to 0 Mvar 
Fig.5.22 shows the system response when the load demand changes back to 0 Mvar at 2s. The reactive power output of $\mathrm{PV}$ and battery reduces to 0 at $2 \mathrm{~s}$. The system restores to $\mathrm{PV}$ dominate mode with the dynamic load switched on and battery operating at 0-RPC mode.

\subsection{5 -1.5 Mvar load step change}
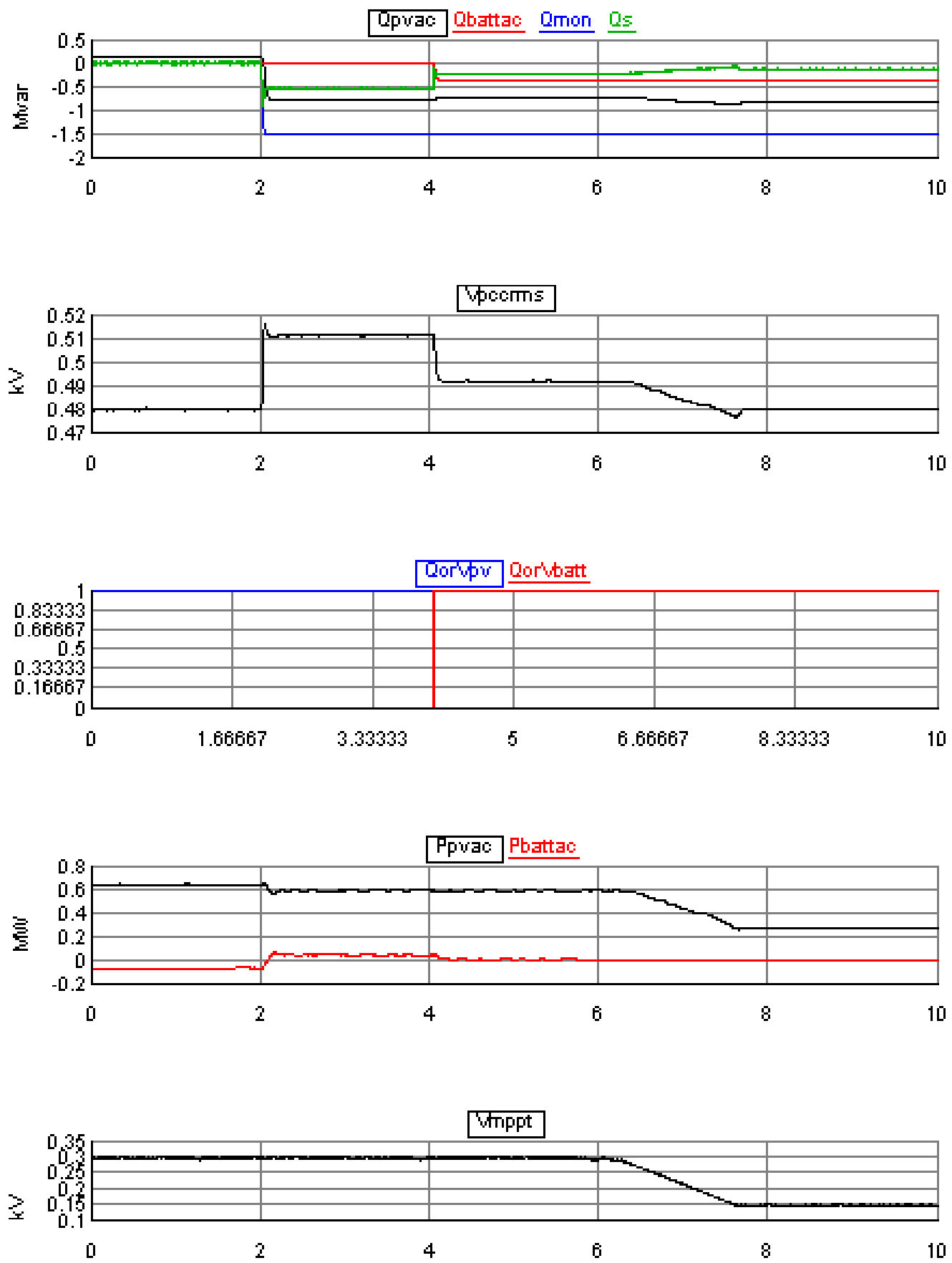

Fig.5.23 Simulation results of the hybrid system under -1.5 Mvar load step change 
A constant insolation $800 \mathrm{~W} / \mathrm{m}^{2}$ is given as the input of $\mathrm{PV}$ array and the hybrid system is initially working under PV dominate mode. A 0 to -1.5 MVAR step change is applied to the dynamic load at $2 \mathrm{~s}$, and the system performance under this load step change is shown in Fig.5.23.
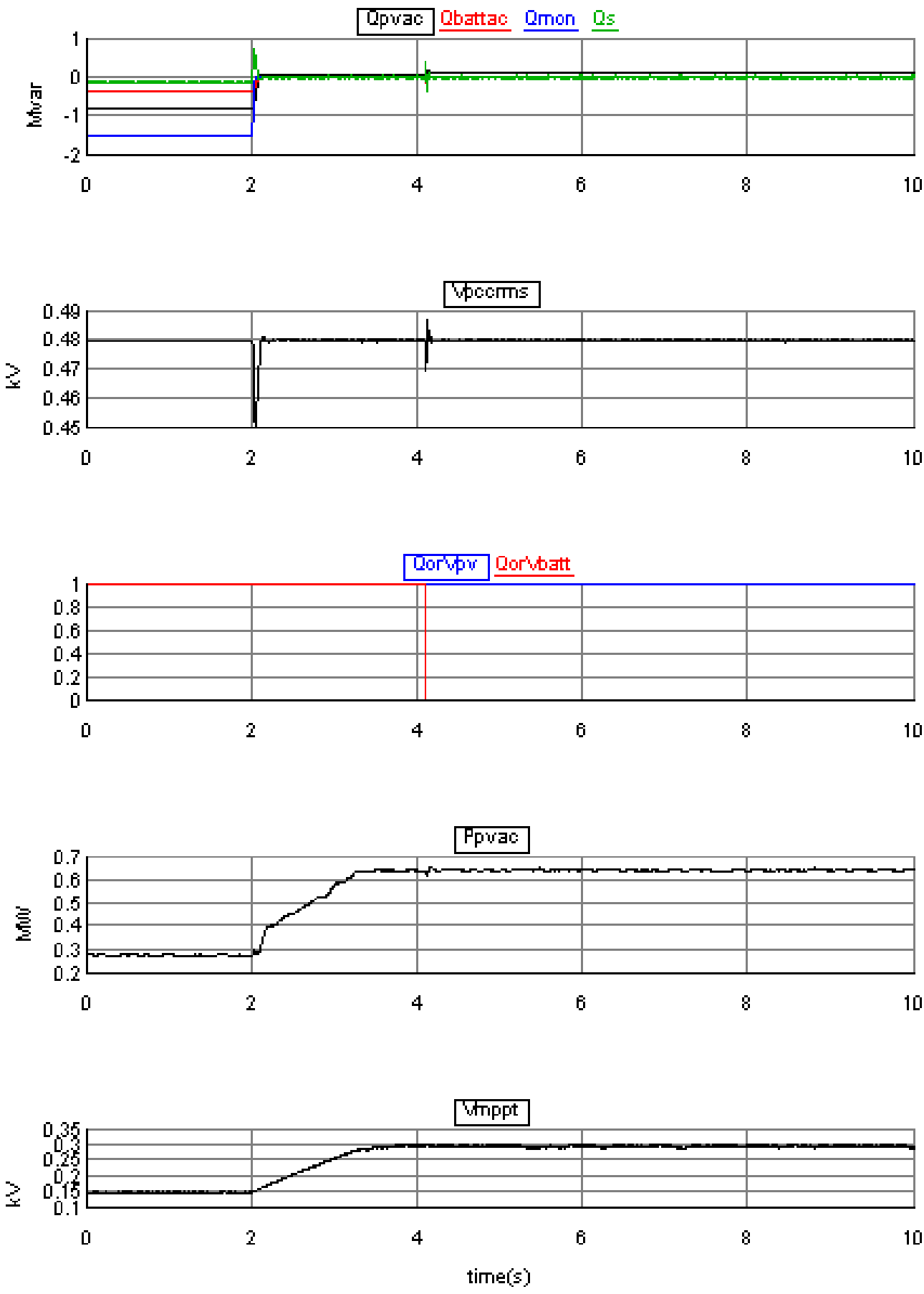

Fig.5.24 Simulation results of the hybrid system when the load changes back from -1.5 Mvar to 0 Mvar 
where Vmppt is the terminal voltage of PV panel. Fig.5.23 shows that Vpccrms violates the high voltage limit even when PV is absorbing maximum reactive power at -0.48 Mvar. A voltage drop is observed when the hybrid system switches to PV and Battery mode at 4s. However, Vpccrms is still violating the high voltage limit with both PV and Battery absorbing maximum reactive power. RMPPT mode is then activated to reduce the active power output of PV panel as well as release extra capacity to absorb reactive power. Vmppt is gradually reducing and Ppvac is changing in the same pattern. In addition, Qpvac is changing from 0.48 Mvar to 0.8 Mvar and Vpccrms is then restored to rated value.

Fig.5.24 shows the system response when the load changes back to 0 Mvar at 2s. The reactive power output of PV and battery reduces to near 0 at $2 \mathrm{~s}$, and RMPPT is then deactivated. The system is restored to PV dominate mode with PV working at maximum power active power output and battery operating at 0-RPC mode. 

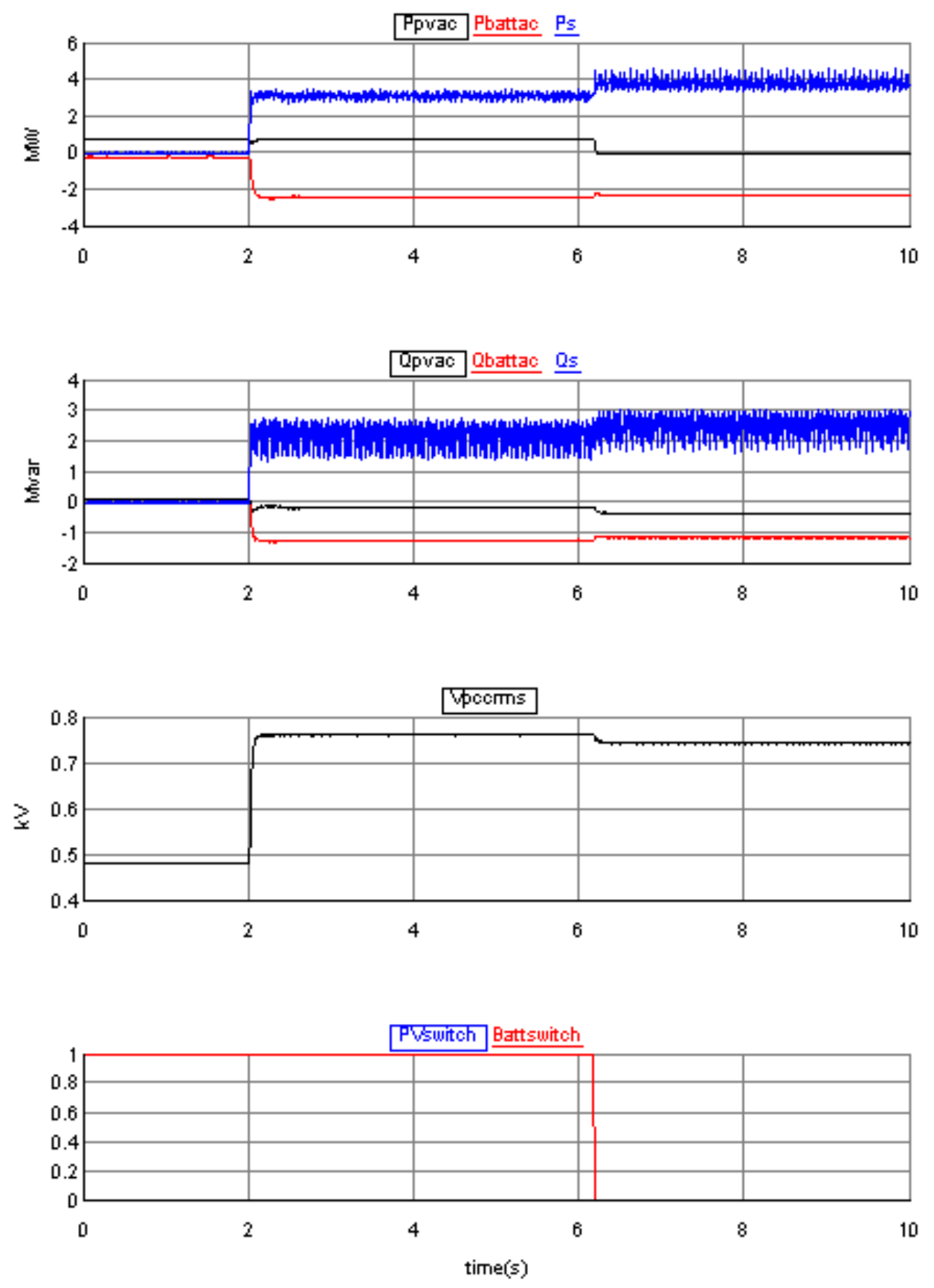

Fig.5.25 Simulation result of the hybrid system under over voltage

\subsubsection{System under over voltage}

The system is initially working under PV dominate mode. A system over voltage is simulated at 2s, the performance of the hybrid system is shown in Fig.5.25, where PVswitch and Battswitch is the status of the PV and battery switch respectively (0: open, 1: close). It is seen that the system voltage violates the voltage protection limit even when the system goes from 
PV dominate mode to PV and Battery mode, and then to RMPPT mode. The protection mode is then activated which disconnects the PV farm and battery storage from the grid to prevent the PV and battery system from physical damage.

\subsection{Hardware Implementation}

Fig.5.26 shows the one line diagram of a microgrid system that consists of a weak distribution feeder, a grid-connected PV system, a grid-connected battery system and two different types of loads: a fixed impedance load and a controllable fixed power load. The PV system consists of a physical PV panel, a digital DC-DC booster and a DC-AC inverter. The battery system consists of a physical lithium-ion storage unit and a digital DC-AC inverter. An infinite voltage source in series with a high inductor is used to represent a weak electric grid with an $\mathrm{R} / \mathrm{X}$ ratio of $1 / 4$. Physical PV panel and battery storage are used and interconnected with RTDS through the Giga -Transceiver Analogue Output (GTAO) and Giga -Transceiver Analogue Input (GTAI) cards which are optically isolated from external hardware for protection. The physical setup of PV panel and Li-ion battery storage is shown in Fig.5.27. The light source above the PV panel is used to simulate sun light, and a dimmer switch is used on the light source to vary the intensity of the light, which is used to effectively simulate real situations of insolation change, such as cloud cover. The load resistor $R$ in the physical system restricts the current from PV panel to exceed the maximum current. 


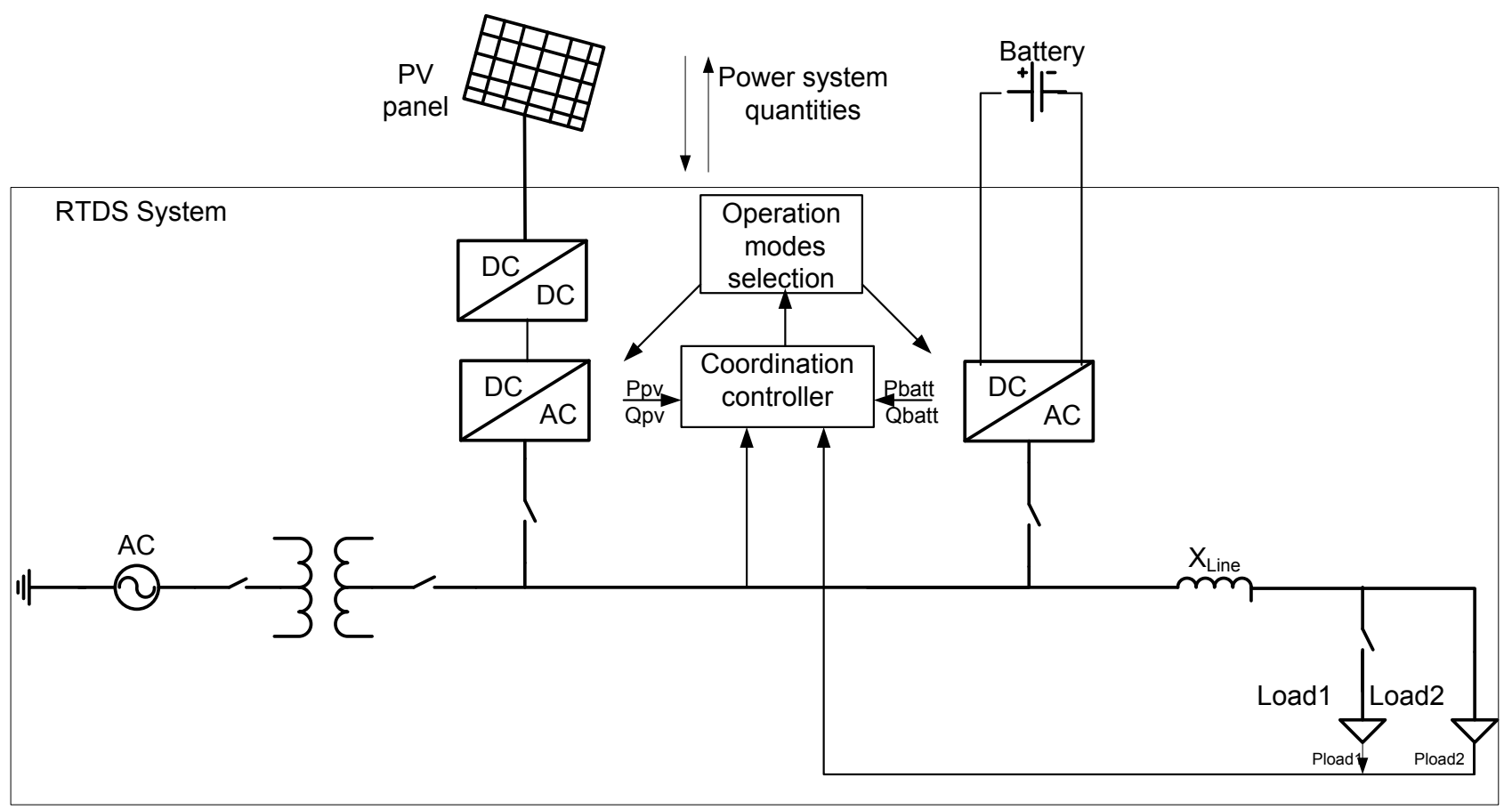

Fig.5.26 One line diagram of the hybrid system 


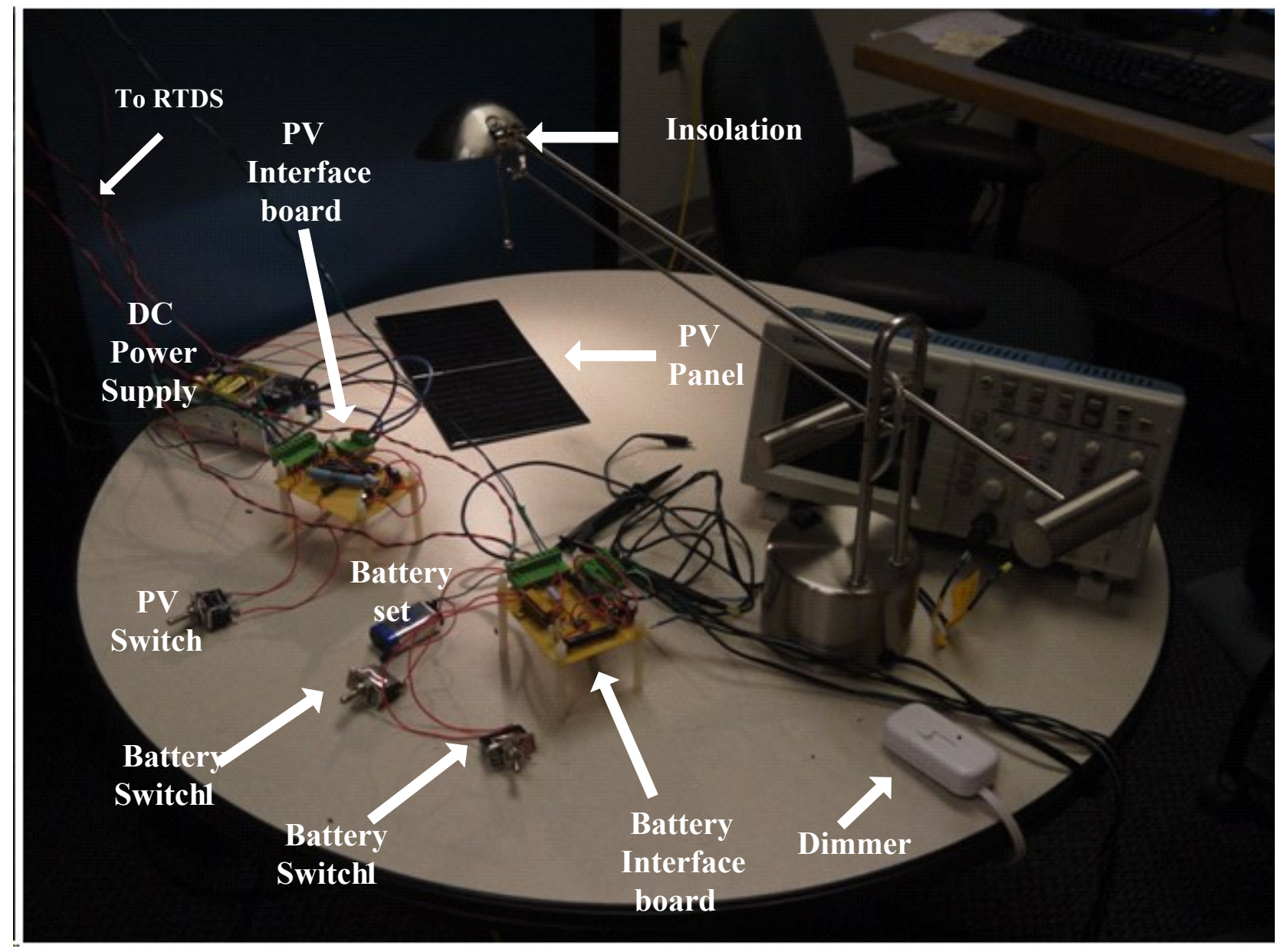

Fig.5.27 Hardware setup of the microgrid

A PV panel can be treated as a controllable current source and the terminal voltage is determined by the insolation level. For the HIL application with RTDS, a controllable current source as shown in Fig.5.28 is used to receive the digital current signal obtained from physical PV panel using the GTAI card. The GTAO card compares the terminal voltage VPV which is scaled down to a physical device rating $V p v$, with the physical PV panel terminal voltage $V p v_{-} a c t$. The error modification controller uses Vpvctrl to regulate the OPA551PA operational amplifier. The OPA551PA operates as a voltage-follower circuit to control the output voltage of the PV panel so that $V p v_{-} a c t$ is regulated to be equal to $V p v$. Thus, the voltage and current outputs of the controllable current source exactly match the output of the physical PV panel at any instant.

The Li-ion battery interface is similar to the PV panel interface except that the components have different values. The battery can be in charging, discharging or idling modes, and the 
modes are controlled by terminal voltage. An operational amplifier to regulate the battery output power, a Zener diode to regulate output voltage of amplifier for protection, LEDs for indicating charging/discharging are also interfaced as shown in Fig.5.28.

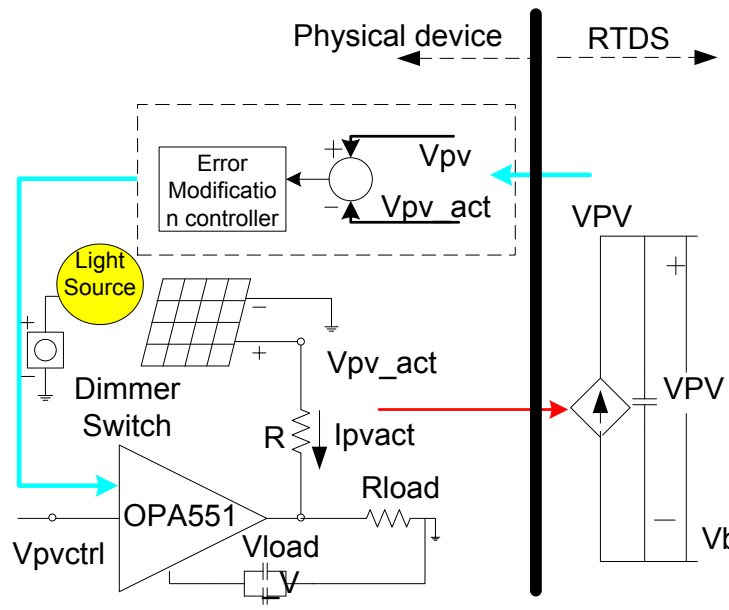

a)

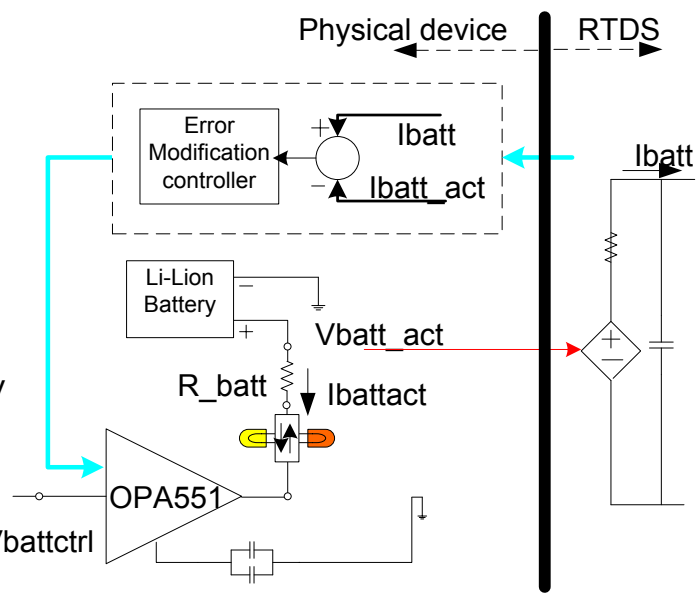

b)

Fig.5.28 Hardware interface board setup a) PV interface; b) Battery interface

\subsection{Experiment Result}

The system in Fig.5.26 is modified to establish a closed loop experimental platform with RTDS and other hardwares as shown in Fig.5.26 to verify the proposed coordinated control strategy. The analog quantities from physical PV and battery are amplified to interface with the distribution system in RSCAD. A PV and battery rated $0.7 \mathrm{MW}$ and $0.3 \mathrm{MW}$ respectively are interfaced with the distribution system. The fixed load is 0.5MVA with 0.95 lag, and the initial dynamic load is $0.1 \mathrm{MW}$. Several scenarios are simulated and the experiment results are presented in this section.

\subsubsection{Interface test}

An insolation change is applied using the light dimmer in hardware interface as shown in Fig.5.28. The voltage and current profiles on both sides of the PV interface before and after insolation change are shown in Fig.5.29. The subscripts cell, act, $p v$, and batt denote the digital, actual, PV panel, and battery unit, respectively. It can be seen that power generated by physical PV panel and battery are accurately simulated by RTDS and the controls are reflected on the physical devices. 

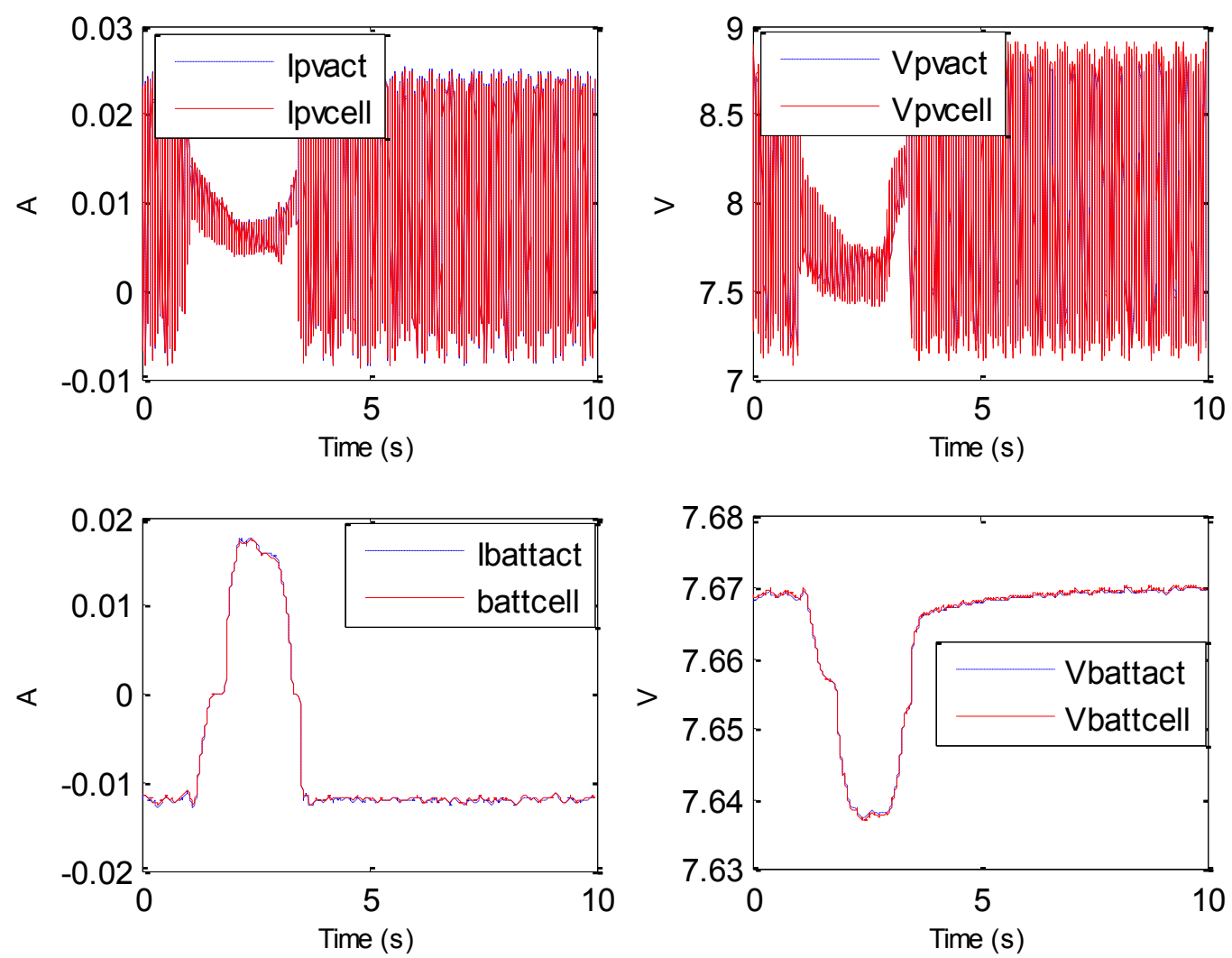

Fig.5.29 Interface test during insolation change 

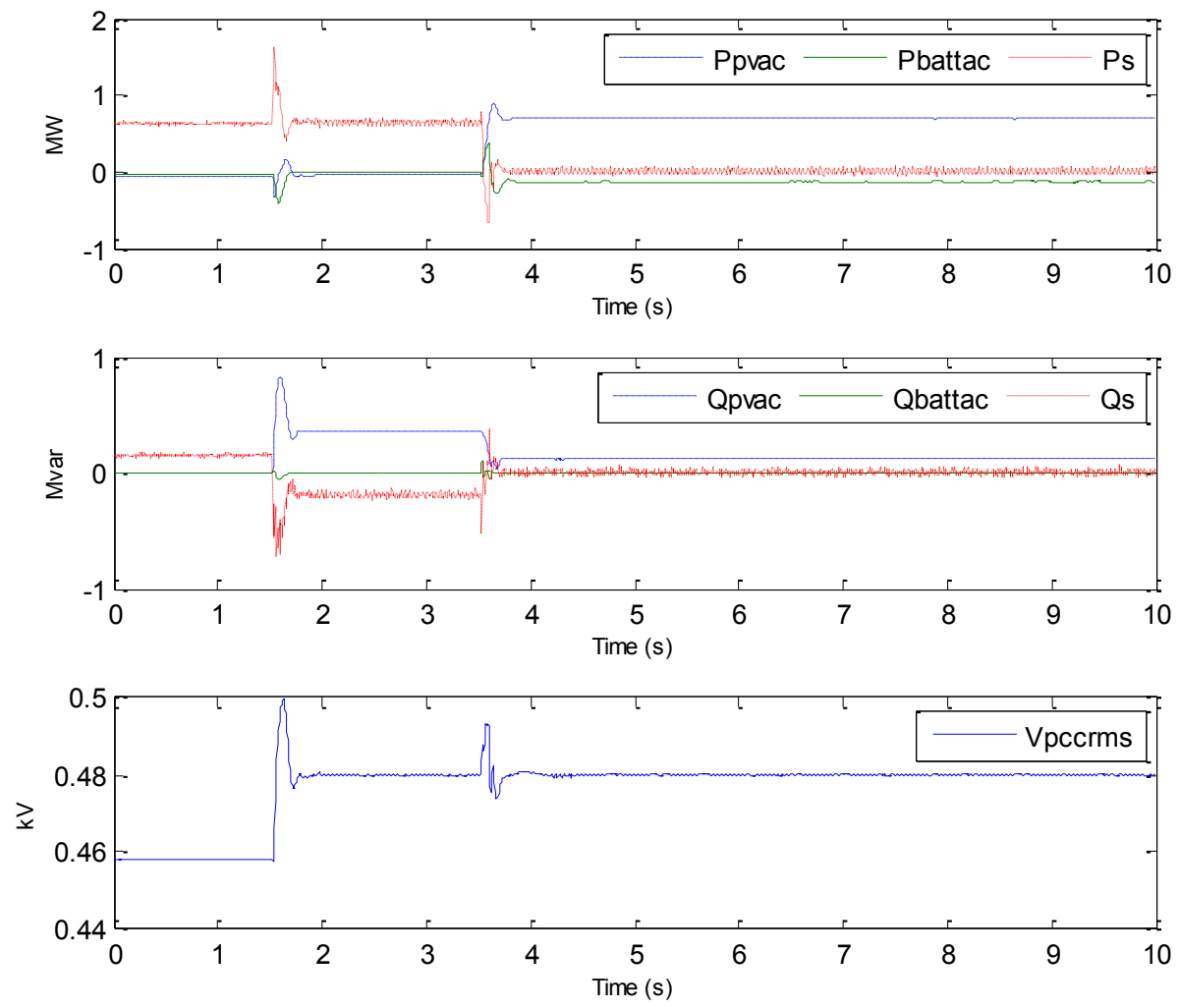

Fig.5.30 System performance during initialization progress

\subsubsection{Initialization progress}

Initially, the microgrid is operating in starting mode with PCC bus voltage Vpccrms as $0.458 \mathrm{kV}$. The PV panel is switched on at 0s under constant insolation. Fig.5.30 plots the active and reactive power delivered by PV farm (Ppvac, Qpvac) and battery (Pbattac, Qbattac), and PCC bus voltage (Vpccrms). After the initialization, the system transitions to PV dominant mode which results in increase in Qpvac from OMVAR to 0.36MVAR to maintain Vpccrms at $0.48 \mathrm{kV}$. $\mathrm{PV}$ and battery maintain power balance with no power exchange with the main power system.

\subsubsection{Insolation change}

The active power coordination during a transient insolation change is tested for insolation sag simulated by the adjustment of light intensity. Fig.5.31 shows the active power sag of the PV farm observed during the insolation sag change which is similar to light intensity change. Using the designed coordinated control, the battery maintains the power balance in the system. The hybrid system operates in PV dominant mode with PV farm providing dynamic voltage support to maintain the PCC bus voltage at $0.48 \mathrm{kV}$. 

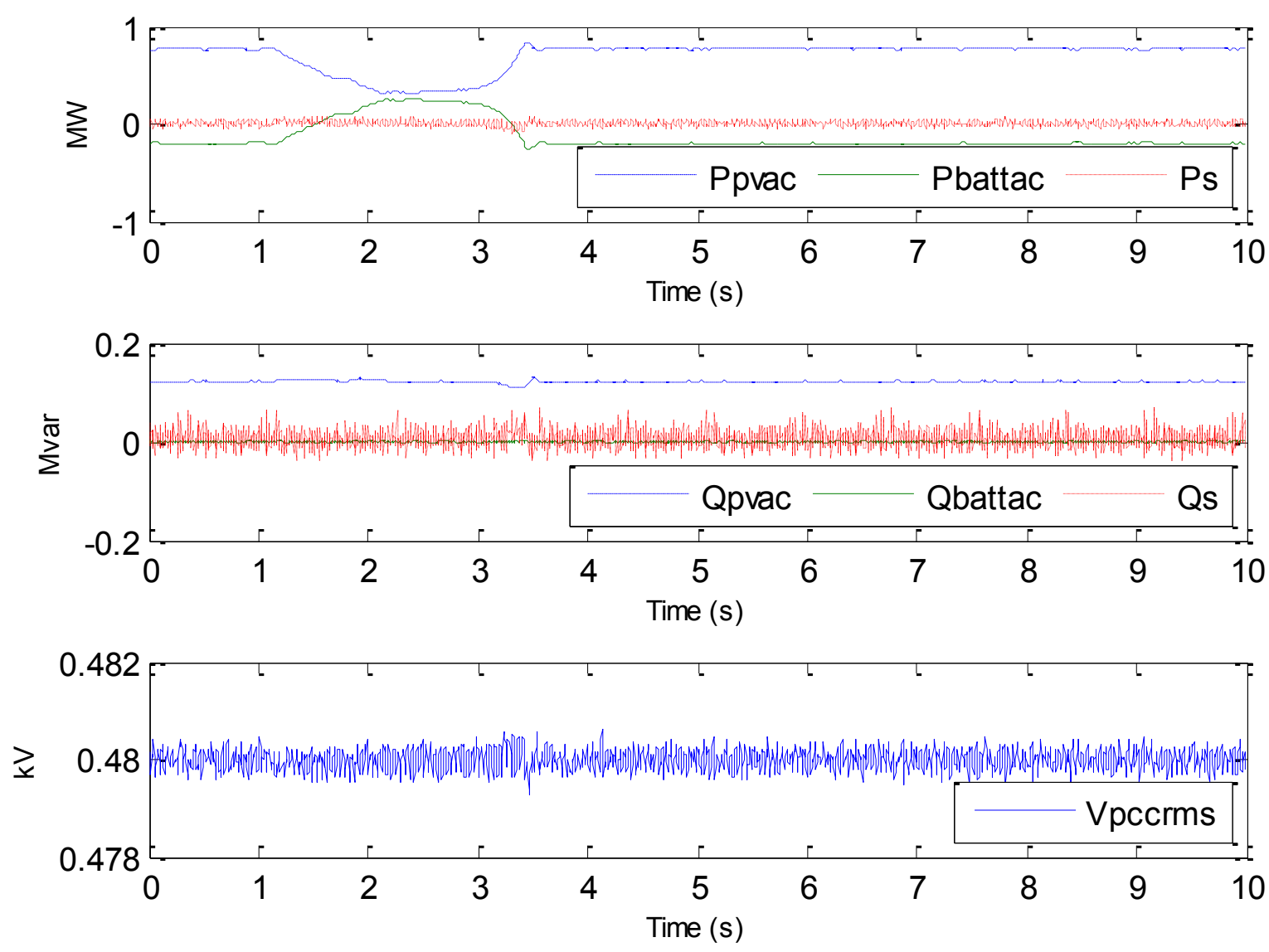

Fig.5.31 System Performance during insolation change

\subsection{MVAR load step change}

Fig.5.32 plots the PCC bus voltage, operation mode (0: 0-RPC, 1: Voltage control) and real power during a $0.5 \mathrm{Mvar}$ load step change and constant insolation. The hybrid system is initially operating under PV dominant mode with Vpccrms at $0.48 \mathrm{kV}$. A $0.5 \mathrm{Mvar}$ inductive load step change at 2 s results in Vpccrms violating the low voltage limit and an increase in reactive power output of the PV inverter Qpvac from 0.1MVAR to its maximum capacity of 0.48MVAR. The hybrid system transitions to PV and battery mode which increases the battery reactive power output Qbattac from 0 to $0.38 \mathrm{MVAR}$ and restores $V p c c r m s$ to $0.48 \mathrm{kV}$. PV and battery maintain the power balance in the microgrid with zero power exchange with the main grid. 

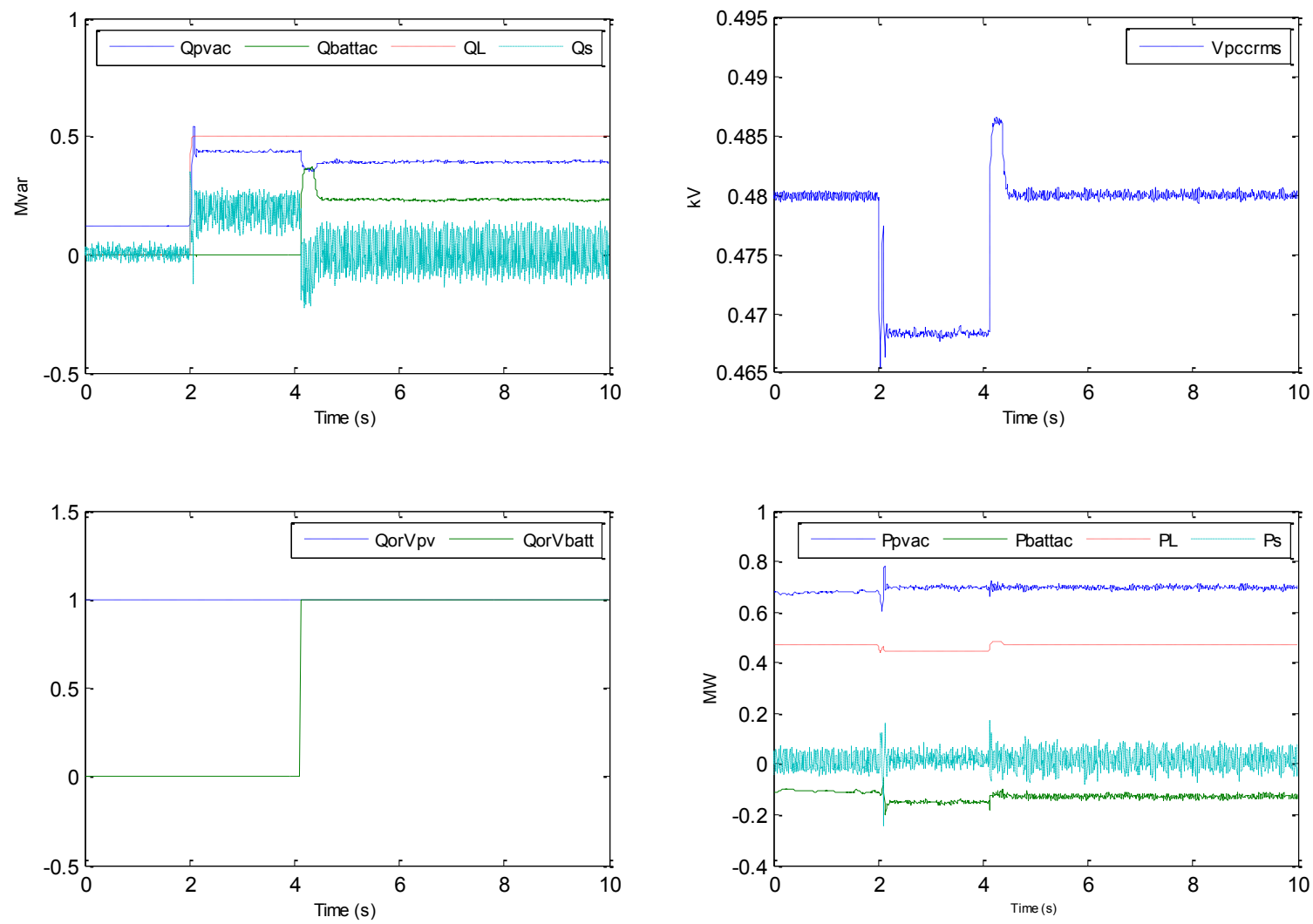

Fig.5.32 System performance under 0.5MVAR load step change

\subsection{MVAR load step change}

Fig.5.32 shows the system performance during a 0.8MVAR load step change and constant insolation. Initially, the system is operating in PV dominant mode with Vpccrms at $0.48 \mathrm{kV}$. The light intensity is maintained as constant and a 0.8MVAR inductive load step change at $2 \mathrm{~s}$ results in Vpccrms violating the low voltage limit and an increase in reactive power output of the PV inverter Qpvac from 0.1MVAR to its maximum capacity of 0.48MVAR. The hybrid system transitions to PV and battery mode which improve the voltage profile but cannot remove violations even though battery reaches its maximum reactive power output. The system transitions to shedding mode where the dynamic load is disconnected at $6.1 \mathrm{~s}$ to restore Vpccrms to $0.48 \mathrm{kV}$. 

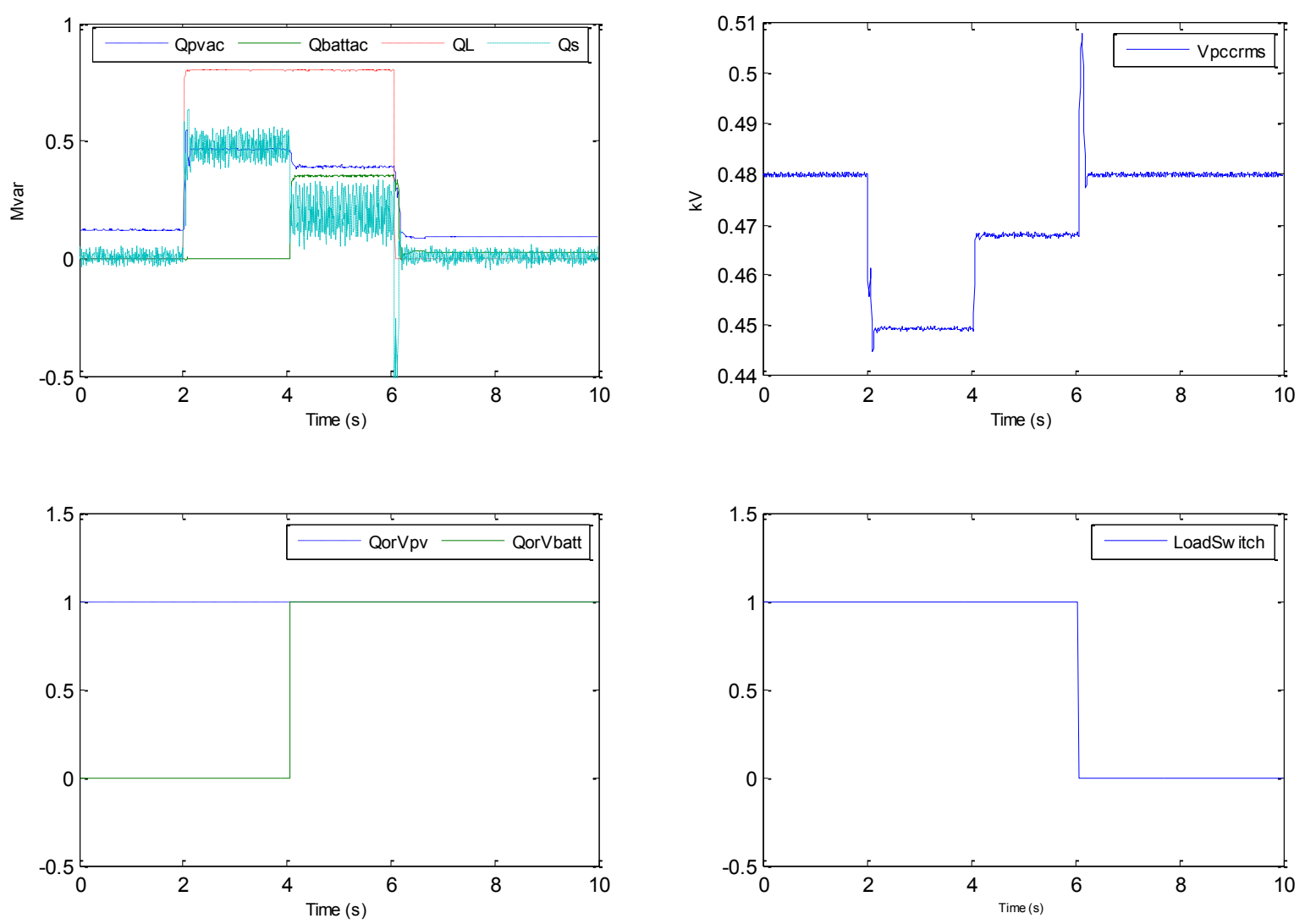

Fig.5.33 System performance during 0.8MVAR load step change

\subsection{MVAR load step change}

A capacitive load step change of 1.5Mvar occurs at 2 s results in Vpccrms violating high voltage limit, the reactive power of PV farm decreases to -0.48MVAR as shown in Fig.5.34. The system transitions to PV and battery mode which improves the voltage profile but voltage violations still exist with battery at its maximum reactive power output. The system transitions to RMPPT mode at 6.3s, where the PV panel voltage Vmppt gradually decreases which results in decrease in the PV active power output Ppvac and decrease in Qpvac from --0.48MVAR to $0.8 \mathrm{MVAR}$ and restoring of Vpccrms to $0.48 \mathrm{kV}$. 

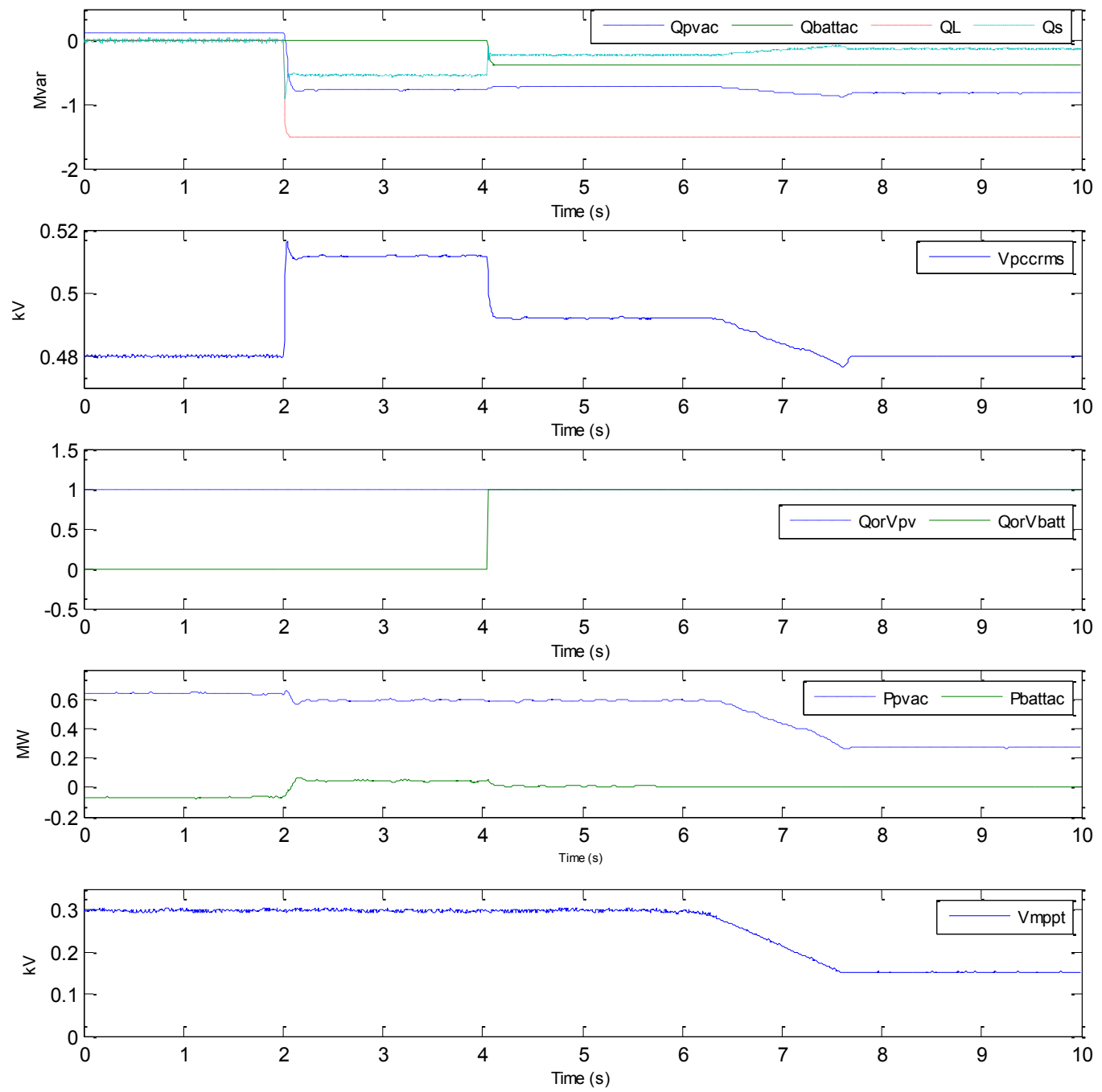

Fig.5.34 System performance under -1.5MVAR load step change

\subsection{Chapter Conclusion}

In this section, the modeling and control of a microgrid with PV and battery interconnections is presented and a genetic algorithm approach is adopted to obtain the optimum PI parameters. A comprehensive coordinated control strategy is proposed to resolve the active power intermittency as well as provide optimal dynamic reactive power support. A novel real-time experimental method for connecting physical PV panel and battery storage is also proposed to establish a HIL microgrid system consisting of physical PV system and battery storage for control strategy test. Through extensive theoretical analysis, computer simulations, and hardware experiment test, the following conclusions are obtained. 
1) With the proposed coordinated control strategy, a battery storage system adds an extra degree of flexibility to a Microgrid by allowing the temporal separation between generation and consumption of power. This strategy increases the stability range of the Microgrid without the need for an additional reactive power compensator.

2) The interface method is feasible and effective, and the output power of physical device and the digital model exactly match.

3) The proposed coordinated control can prevent the system from voltage collapse by providing three levels of protection: PV voltage control mode, PV and battery voltage control mode, and load shedding and RMPPT mode. This three level protection optimizes PV and battery inverter performance without influencing the life span of battery storage. The protection mode also protects the PV and battery by disconnecting PV and battery from the grid when the system voltage is over or under specified limits.

4) The coordinated control scheme can be used as a general application for microgrids with PVs, wind turbines and batteries. Further integration studies and control strategies can be extended to frequency deviations due to large changes in load and generation. 


\section{Chapter 6. Coordinated Predictive Control of a Wind/Battery Microgrid system}

A MPC based coordinated control scheme is designed that enables the microgrid to provide sustainable power as well as dynamic reactive power support to the load in both gridconnected and islanded operation, thus reducing active power oscillation as well as tracking maximum wind energy. The schematic representation of the microgrid system is shown in Fig.6.1. The system is composed of a VSCF wind generator, battery storage and two different kinds of load: a fixed impedance load and a dynamic load with controllable power. Wind generator consists of a gearless direct drive wind turbine, a multi pole Permanent Magnet Synchronous Generator (PMSG), and an AC-DC-AC converter. The battery system consists of a lithium-ion storage unit and a DC-AC inverter. An infinite voltage source in series with a high inductor is used to represent a weak electric grid. The controller design is introduced in the following sections.

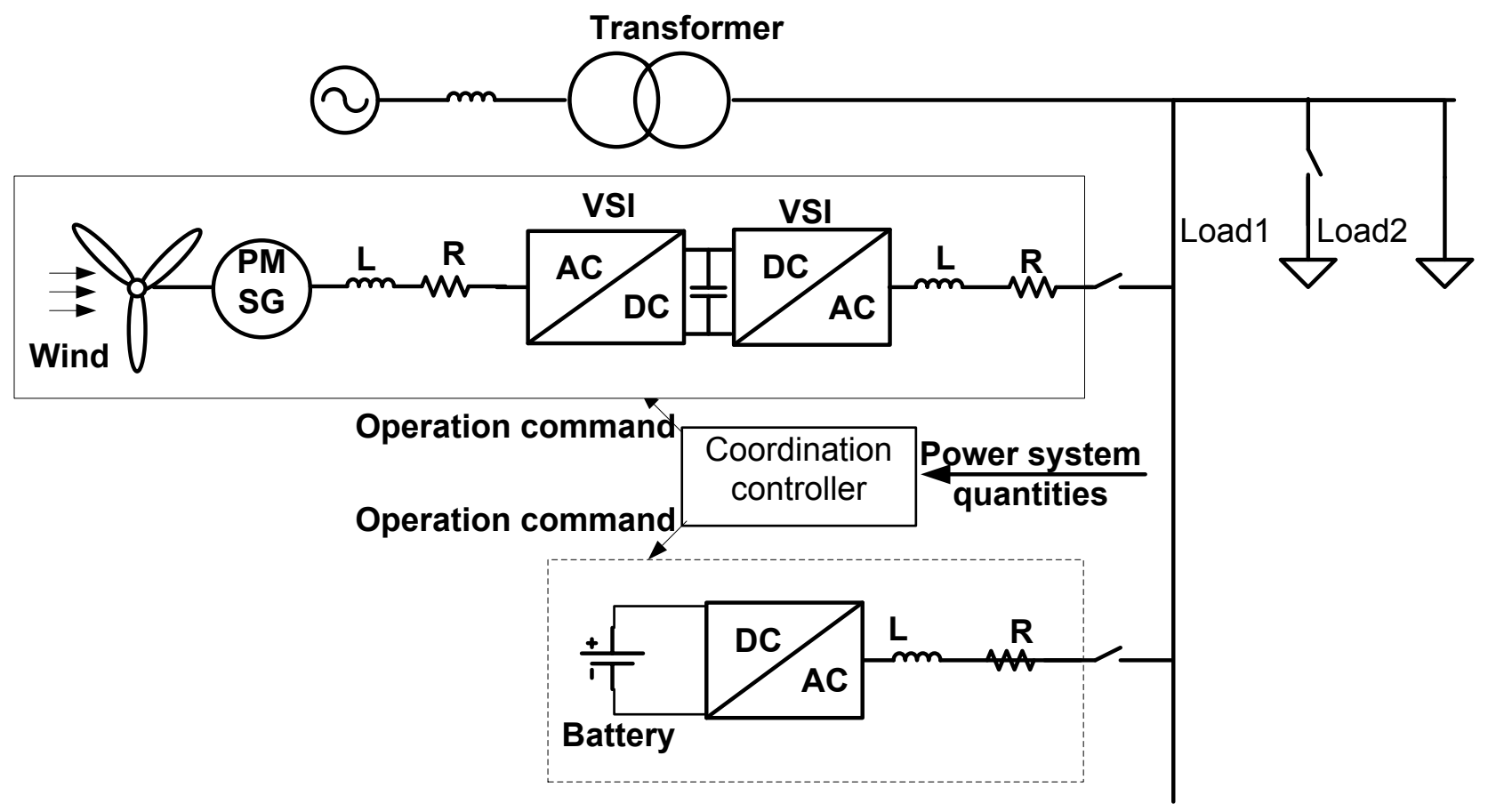

Fig.6.1 One line diagram of the microgrid

\subsection{MPC Strategy of a Wind AC-DC-AC Inverter}


The modeling of a wind turbine introduced in [102] is adopted in this paper. However, instead of using diode rectifier, an Insulated Gate Bipolar Transistor (IGBT) based AC-DC-AC inverter is used as the interface between wind turbine and grid as shown in Fig.6.2,

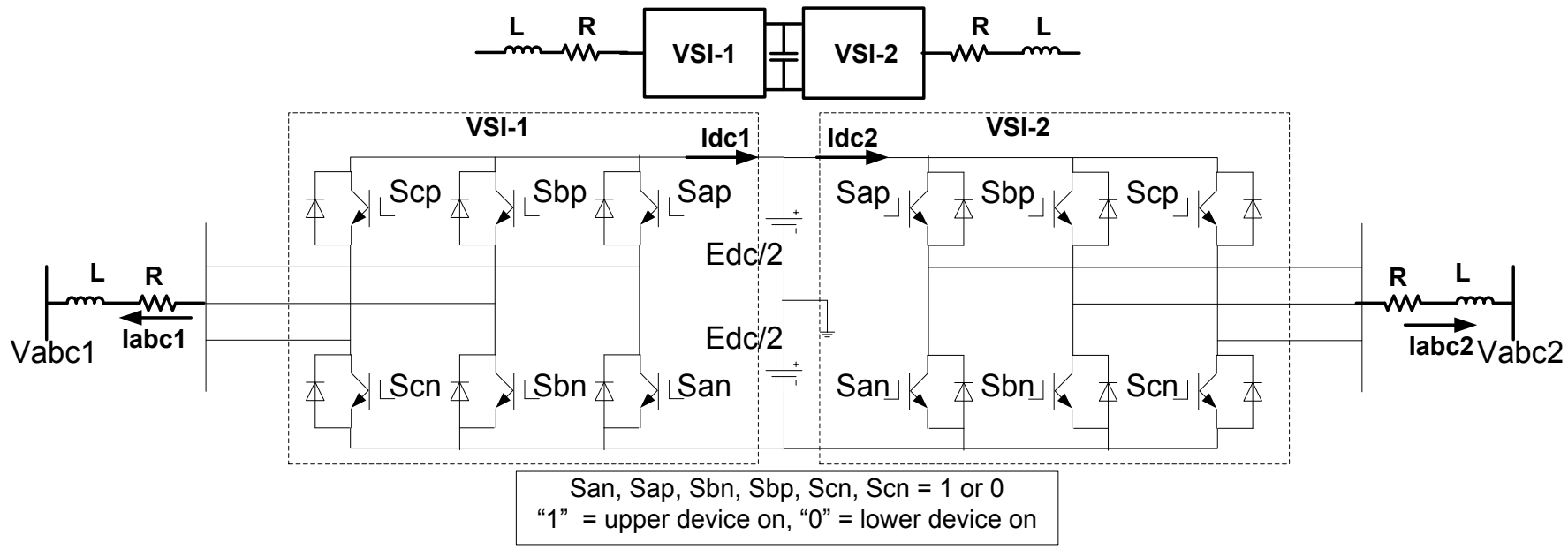

Fig.6.2 circuit diagram of wind converter

\subsubsection{Basic operation of AC-DC-AC inverter}

A six IGBT bridge Voltage Source Inverter (VSI) is located on both sides of the DC bus as shown in Fig.6.2. In a 2-level VSI used in this paper, there are a total of $C_{2}^{1}$ available switching states in each phase [81]. The switching function of phases $j=a, b, c$ of VSI-k $(k=1,2)$ are controlled so that at any instant only one bridge is switched on for each phase, which means either the upper or lower arm is on. Taking advantage of the limited switching states, the desired switching states can be obtained to track the maximum wind energy and provide desired reactive power.

\subsubsection{Mathematical model of a VSI}

Assuming the three phase voltage is balanced, the dynamic behavior of VSI-k phase $j$ can be expressed as in equations (6.1) and (6.2),

$$
\begin{array}{r}
V_{j k}+L \frac{d i_{j k}}{d t}+R i_{j k}+V_{p j k}=\frac{E_{d c}}{2} \\
V_{j k}+L \frac{d i_{j k}}{d t}+R i_{j k}-V_{n j k}=-\frac{E_{d c}}{2}
\end{array}
$$

where $V$ and $i$ are the voltage and current of VSI, respectively, $V_{p}$ and $V_{n}$ are the upper and lower bridge voltages, respectively, $j k$ denote phase- $j$ of VSI- $k$, and $E_{d c}$ is the dc bus voltage. Adding (6.1) with (6.2), the phase voltage of VSC can be expressed as, 


$$
\frac{d i_{j k}}{d t}=\frac{V_{n j k}-V_{p j k}-2 R i_{j k}-2 V_{j k}}{2 L}
$$

Based on the switching function, $V_{p j k}$ and $V_{n j k}$ can be represented as,

$$
\left\{\begin{array}{l}
{\left[\begin{array}{l}
V_{p j k} \\
V_{n j k} \\
S_{n j k}
\end{array}\right]=\left[\begin{array}{c}
0 \\
E_{d c} \\
0
\end{array}\right], S_{p j k}=1} \\
{\left[\begin{array}{l}
V_{p j k} \\
V_{n j k} \\
S_{n j k}
\end{array}\right]=\left[\begin{array}{c}
E_{d c} \\
0 \\
1
\end{array}\right], S_{p j k}=0}
\end{array}\right.
$$

where, $S_{p}$ and $S_{n}$ represent the switching states of upper and lower bridge arm, respectively. In PSCAD, 1 is close, and 0 is open. Note that, different softwares use different representations. The dc bus voltage $E_{d c}$ can be calculated as,

$$
i_{d c 1}-i_{d c 2}=C \frac{d E_{d c}}{d t}
$$

\subsubsection{MPC strategy}

The objectives of the generator side VSI are to regulate the ac current to capture maximum wind energy as well as maintain zero reactive power exchange. MPC strategy is so designed to obtain the next time step switching states based on the forecasted wind speed and the

dynamic behavior of VSI. Assuming the next time step wind speed is known at next time instant, the wind generation and torque with respect to wind speed can be described as $[103,104]$,

$$
\begin{aligned}
& P_{\text {wind_opt }}=\frac{1}{2} \rho \pi R^{2} C_{p_{-} \_p t} V_{w}^{3} \\
& T_{w}=\frac{P_{w_{-} o p t}}{\omega_{w}}=\frac{1}{2} \rho \pi R^{2} C_{p_{-} o p t} \frac{V_{w}^{3}}{\omega_{w}}
\end{aligned}
$$

where $\rho$ is the air density, $R$ is the blade radius, $V_{w}$ is the wind speed, $\omega_{w}$ is the mechanical speed of wind turbine, $T_{w}$ is the mechanical torque of wind turbine, and $C_{p}$ is the power coefficient and it is a function of $\omega_{w}, V_{w}$ and blade pitch angle $\beta . C_{p_{-} o p t}$ is the optimum power coefficient and captures the maximum wind energy $P_{\text {wind_opt }}$. The approximation of $C_{p_{-} o p t}$ used in this paper can be found in $[105,106]]$.

The power exchange between wind turbine and generator side VSI in next time step can be expressed in synchronous dq0 reference frame as, 


$$
\left[\begin{array}{l}
P\left(t+T_{s}\right) \\
Q\left(t+T_{s}\right)
\end{array}\right]=\left[\begin{array}{c}
\frac{1}{2} \rho \pi R^{2} C_{p} V_{w}^{3}\left(t+T_{s}\right) \\
0
\end{array}\right]=\left[\begin{array}{l}
\frac{3}{2} V_{q}\left(t+T_{s}\right) I_{q}\left(t+T_{s}\right) \\
\frac{3}{2} V_{q}\left(t+T_{s}\right) I_{d}\left(t+T_{s}\right)
\end{array}\right]
$$

where $d$ and $q$ denote the $d$-axis and $q$-axis sequence, respectively. $T_{S}$ is the time step. By selecting a sufficiently small $T_{s}, V_{q}\left(t+T_{s}\right)$ can be approximated by the measured value of $V_{q}(t)$ [61]. It is seen that the active and reactive power $P\left(t+T_{s}\right)$ and $Q\left(t+T_{s}\right)$ can be obtained by regulating $I_{q}\left(t+T_{s}\right)$ and $I_{d}\left(t+T_{s}\right)$. The reference $a b c$ current of next time step can be calculated by the PI controller as shown in Fig.6.3. Similarly, the objectives of the grid side VSI are to deliver desired reactive power to the grid as well as maintain the dc bus voltage. The reference current of grid side VSI can be obtained in similar manner as shown in Fig.6.3. The phase tracking system presented in [107] is applied in this paper to get the synchronous rotating speed.

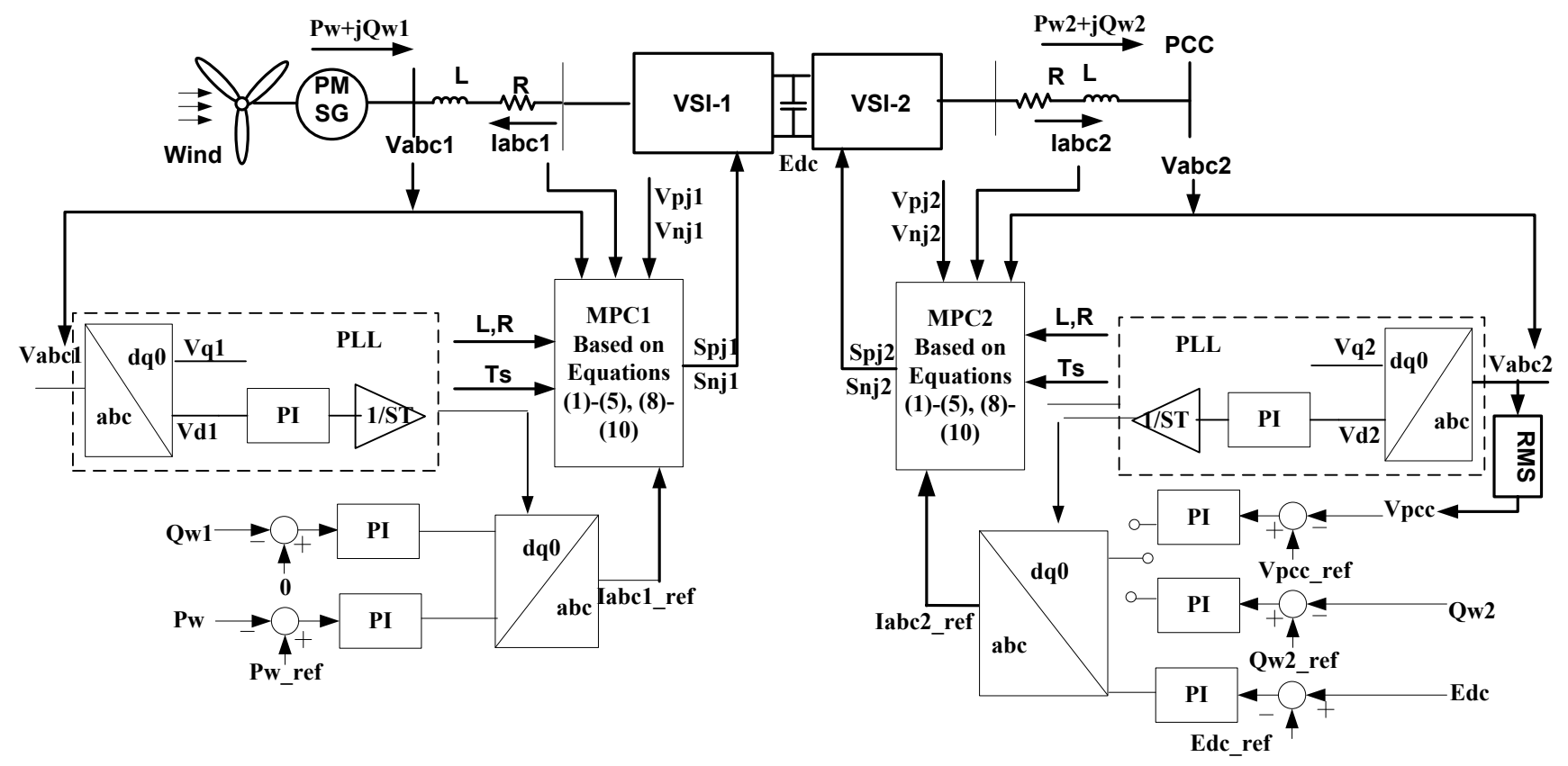

Fig.6.3 Control scheme of wind converter

\subsubsection{MPC formulation}

An accurate prediction of next time step current is essential for tracking. Based on equation (6.3), the dynamic discrete-time model of generator side VSI can be deduced with a backward Euler approximation as shown in equation (6.9),

$$
i_{j k}\left(t+T_{s}\right)=\frac{L}{L+R \times T_{s}} i_{j k}(t)+T_{s} \times \frac{V_{n j k}\left(t+T_{s}\right)-V_{p j k}\left(t+T_{s}\right)}{2 L+2 R \times T_{s}}-T_{s} \times \frac{V_{j k}\left(t+T_{s}\right)}{L+R \times T_{s}}
$$


To reduce the error between predicted and reference current, a cost function is presented to evaluate the predicted current under all combinations of switching states as shown in equation (6.10)

$$
J_{j k}=\left|i_{j k r e f}\left(t+T_{s}\right)-i_{j k}\left(t+T_{s}\right)\right|
$$

The MPC strategy is applied both on the VSI of the wind generator and VSI of battery system, since the battery is connected to the system through a voltage source inverter similar to the grid-side inverter of direct drive wind turbine. The mathematical model and the MPC formulation of VSI can be used for both wind generator and battery system. Similar strategy is applied to battery inverter as shown in Fig.6.4, and the control objective of battery inverter is set to provide desired power to the grid. The acquisition of the active and reactive power reference for battery system will be described in the following sections.

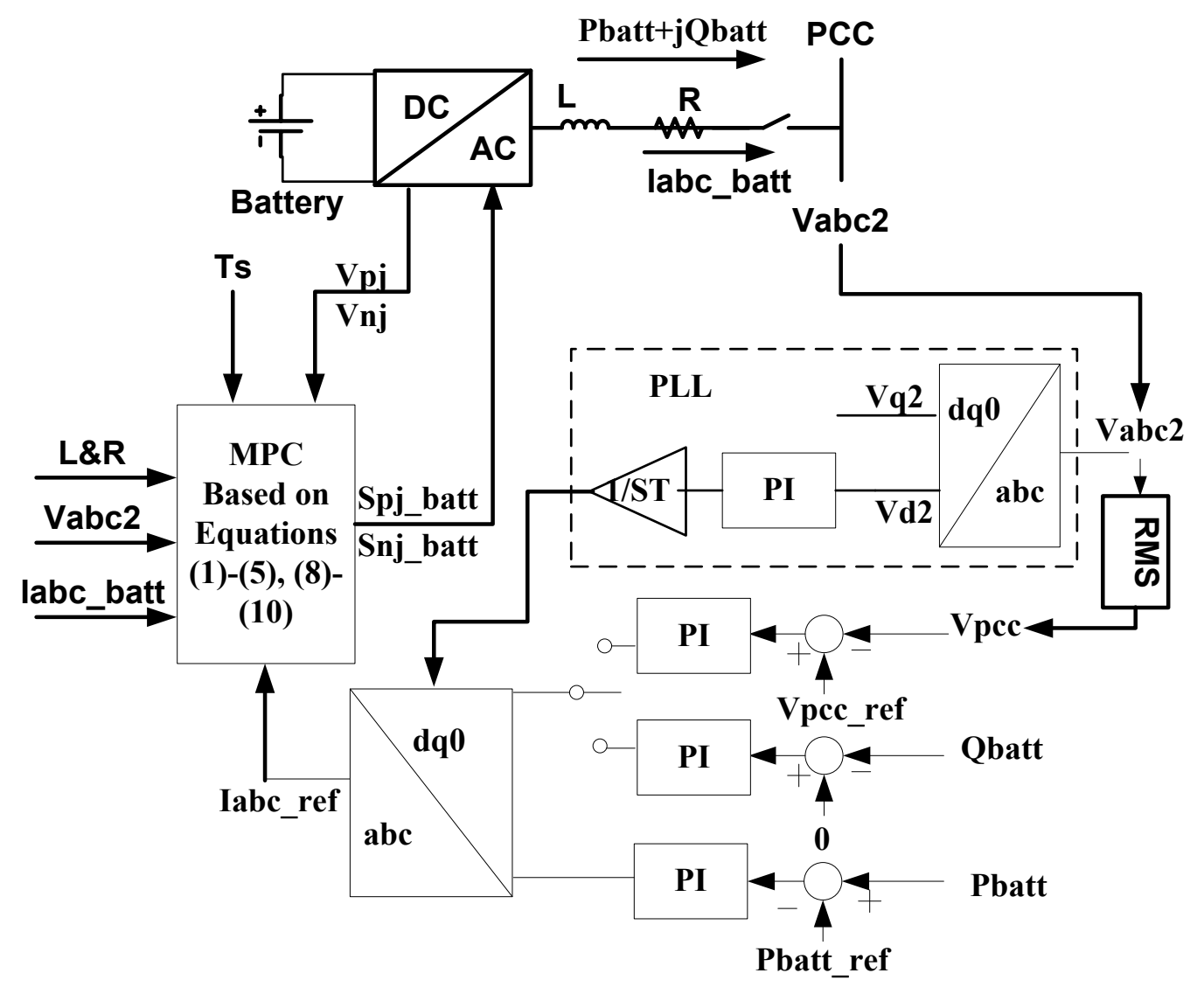

Fig.6.4 Control scheme of battery VSI

\subsection{Microgrid Operation and Coordinated Control Strategy}

The coordinated controller for the microgrid is as shown in Fig.6.5. The monitored power system quantities include PCC bus voltage, real and reactive power output of wind and 
battery as well as the breaker statuses of wind, battery and load. This section presents the reactive and active power coordination under different operation.

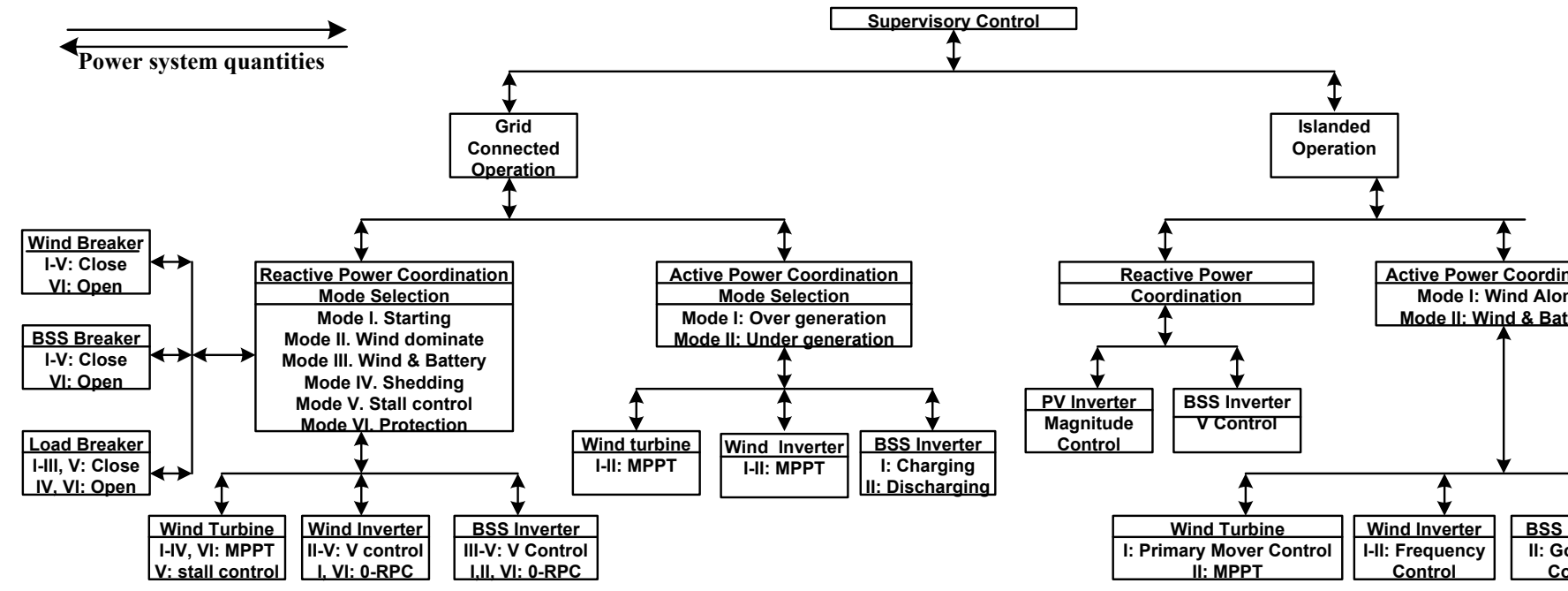

Fig.6.5 Coordinated control scheme of the microgrid with wind and battery storage 


\subsubsection{Grid-connected operation}

- $\quad$ Reactive power coordinated control

In grid-connected mode, the synchronous generators in main power grid maintain the voltage and frequency of the microgrid constant even when there is no power exchange on the interconnected transmission line. However, since the microgrid is normally connected at the remote end of the power system where lack of reactive power support is expected, wind and battery are responsible for providing dynamic reactive power and maintaining the PCC bus voltage. A reactive power coordinated control scheme for grid-connected operation is designed considering the aforementioned criterions as shown in Fig.6.5 for the coordination of wind converter and battery inverter. Six different operation modes are implemented as follows:

1) Start mode: This mode occurs during the system starting procedures. The wind farm starts in zero Reactive Power Control (0-RPC) mode and then switches to voltage control mode in steady state. The battery is connected $2 \mathrm{~s}$ after wind farm starts operating in 0 RPC mode to reduce transients. Wind converter generates reactive power until PCC bus voltage is constant.

2) Wind dominant mode: This mode is activated after the initialization mode where wind farm is the primary reactive power compensator. In this mode the battery is at zero reactive power output regardless of charging or discharging. In the wind dominant mode small transient disturbances are mitigated by the wind farm thereby improving the life cycle of battery storage.

3) Wind and Battery mode: This mode is activated when the PCC voltage violates the high limit and the wind converter reaches its maximum reactive power capability. The battery switches to voltage control mode to provide dynamic reactive power support. When wind and battery both are in voltage control mode, the coordinated control prevents oscillations by utilizing a basic droop [108] in the voltage vs reactive power characteristics of both wind and battery to ensure the voltage change in PCC bus is absorbed by both wind and battery system. The parameters of the controllers are optimized through a genetic algorithm to achieve optimal performance in voltage regulation and active power control. 
4) Shedding mode: This mode is activated when the PCC voltage violates the low limit and both wind farm and battery are at their maximum reactive power output. Under this condition some of the load may be disconnected for voltage recovery.

5) Stall mode: This mode is activated when the PCC voltage violates the high limit and both wind and battery inverters absorb maximum reactive power. The wind turbine goes to stall regulation, and the maximum power control is overridden. The blade pitch angle is increased to reduce the wind energy capture. The active power output of the wind turbine is reduced to increase the maximum reactive power capability of wind converter.

6) Protection Mode: This mode is activated when the PCC voltage violates the protection limits. In order to protect the power electronics device from over voltage or under voltage conditions and other safety issues, both the wind and battery system are disconnected from the power grid.

Time deadband is introduced in this coordinated controller to avoid the excessive mode changes. Table 6.1 gives the control strategy map for each operation mode. With this algorithm, autonomous operation of wind and battery is achieved, providing flexibility of operation.

TABLE 6.1

CONTROL STRATEGY MAP FOR OPERATION MODES UNDER REACTIVE POWER COORDINATE CONTROL

\begin{tabular}{|l|l|l|l|l|l|l|}
\hline $\begin{array}{l}\text { Operation } \\
\text { mode }\end{array}$ & $\begin{array}{l}\text { Wind } \\
\text { Turbine }\end{array}$ & $\begin{array}{l}\text { Wind } \\
\text { inverter }\end{array}$ & $\begin{array}{l}\text { Battery } \\
\text { Inverter }\end{array}$ & $\begin{array}{l}\text { Wind } \\
\text { breaker }\end{array}$ & $\begin{array}{l}\text { Battery } \\
\text { breaker }\end{array}$ & $\begin{array}{l}\text { Dynamic } \\
\text { Load breaker }\end{array}$ \\
\hline Start mode & MPPT & 0-RPC & 0-RPC & Close & Close & Close \\
\hline $\begin{array}{l}\text { Wind } \\
\text { dominant } \\
\text { mode }\end{array}$ & MPPT & $\begin{array}{l}\text { Voltage } \\
\text { control }\end{array}$ & 0-RPC & Close & Close & Close \\
\hline $\begin{array}{l}\text { Wind and } \\
\text { Battery mode }\end{array}$ & MPPT & $\begin{array}{l}\text { Voltage } \\
\text { control }\end{array}$ & $\begin{array}{l}\text { Voltage } \\
\text { control }\end{array}$ & Close & Close & Close \\
\hline $\begin{array}{l}\text { Shedding mode } \\
\text { MPPT }\end{array}$ & $\begin{array}{l}\text { Voltage } \\
\text { control }\end{array}$ & $\begin{array}{l}\text { Voltage } \\
\text { control }\end{array}$ & Close & Close & Open \\
\hline Stall mode & $\begin{array}{l}\text { Stall } \\
\text { regulate }\end{array}$ & $\begin{array}{l}\text { Voltage } \\
\text { control }\end{array}$ & $\begin{array}{l}\text { Voltage } \\
\text { control }\end{array}$ & Close & Close & Close \\
\hline $\begin{array}{l}\text { Protection } \\
\text { Mode }\end{array}$ & MPPT & 0 -RPC & 0-RPC & Open & Open & Open \\
\hline
\end{tabular}

The coordinated control expands the operation range and provides the system four level of protection,

1) Voltage regulation by wind farm only.

2) Voltage regulation by both farm and battery.

3) Load shedding and stall regulation 


\section{4) Wind and battery splitting}

The system can also restore from any operation point to initial conditions if certain requirements are met.

- $\quad$ Active power coordinated control

The uncontrollable nature of wind energy leads to intermittent active power supply and hence unbalance between supply and demand. However, in remote distribution network, it is desirable that distributed generators supply local load. The power imbalance can be mitigated by the active power coordination shown in Fig. 6. Equation (6.11) define the battery active power output,

$$
\mathrm{P}_{\text {wind }}+\mathrm{P}_{\text {batt }}+\mathrm{P}_{\text {grid }}=\mathrm{P}_{\mathrm{t}}+\mathrm{P}_{\text {loss }}
$$

where $P_{\text {wind }}, P_{\text {batt }}$, and $P_{\text {grid }}$ are the active power command of wind, battery and grid, $P_{t}$ is the total load demand, and $P_{\text {loss }}$ is the system loss. The microgrid is so designed that the wind farm and battery can support the total local load, and hence under most conditions, no real and reactive power will be needed from the grid, resulting in low power losses also. The active power from grid is needed when the wind speed is low and the total load $P_{t}$ exceeds the maximum active power generation of $P_{\text {wind }}+P_{\text {batt }}$. After inclusion of $P_{\text {loss }}$ in the equation, no significant change is observed due to negligible transmission losses in the microgrid

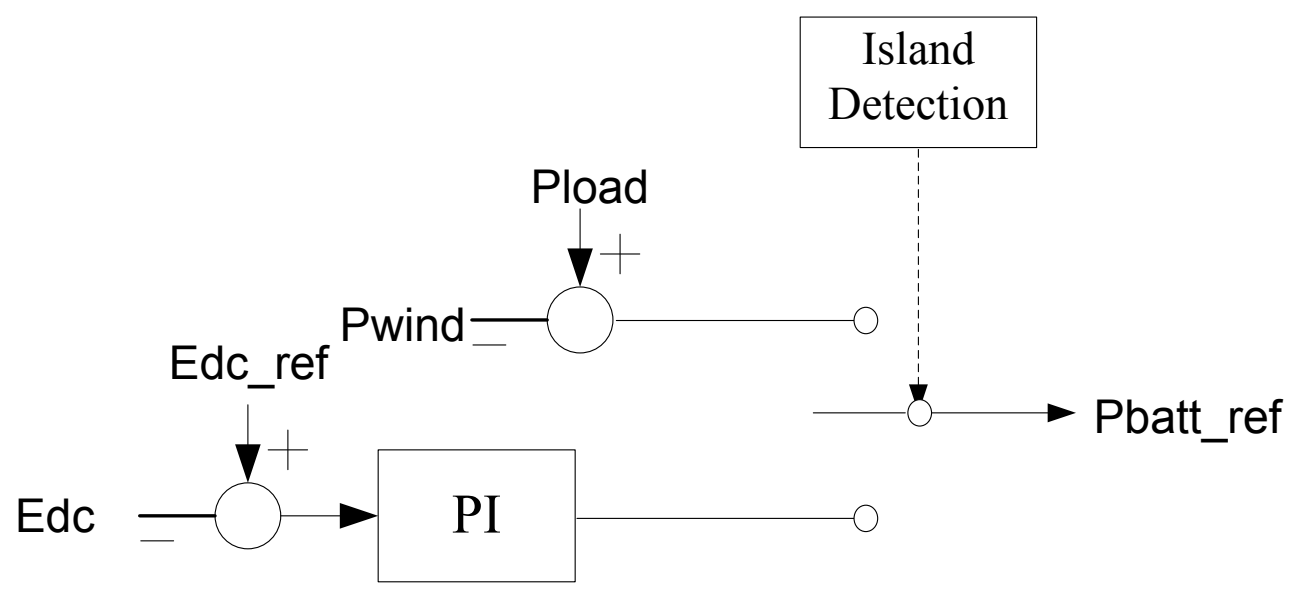

Fig.6.6 Active power coordination

\subsubsection{Island operation}

In a standalone operation, the network is isolated from the main AC supply system. There are no synchronous alternators that can provide voltage and frequency references. In this paper, 
a seamless transition method is proposed as shown in Fig.6.7 to calculate the reference voltage angle for islanded control operation. An ideal three phase sinusoid wave is used as the base reference angle, and the phase angle of PCC voltage before islanding is used as the initial phase angle of the sinusoid wave to minimize the oscillation caused by large angle shift before and after islanding. Wind farm is the primary source of supply in the island. Wind converter generates a $60 \mathrm{~Hz}$ three phase balanced constant voltage signal as reference. A voltage drop or voltage rise at dc link can occur due to fluctuations in wind power. Battery is used to regulate the PCC bus voltage as well as to ensure the maximum power extraction capability of wind farm during islanded operation. However, in certain cases, partial noncritical load will be shed to maintain power balance.

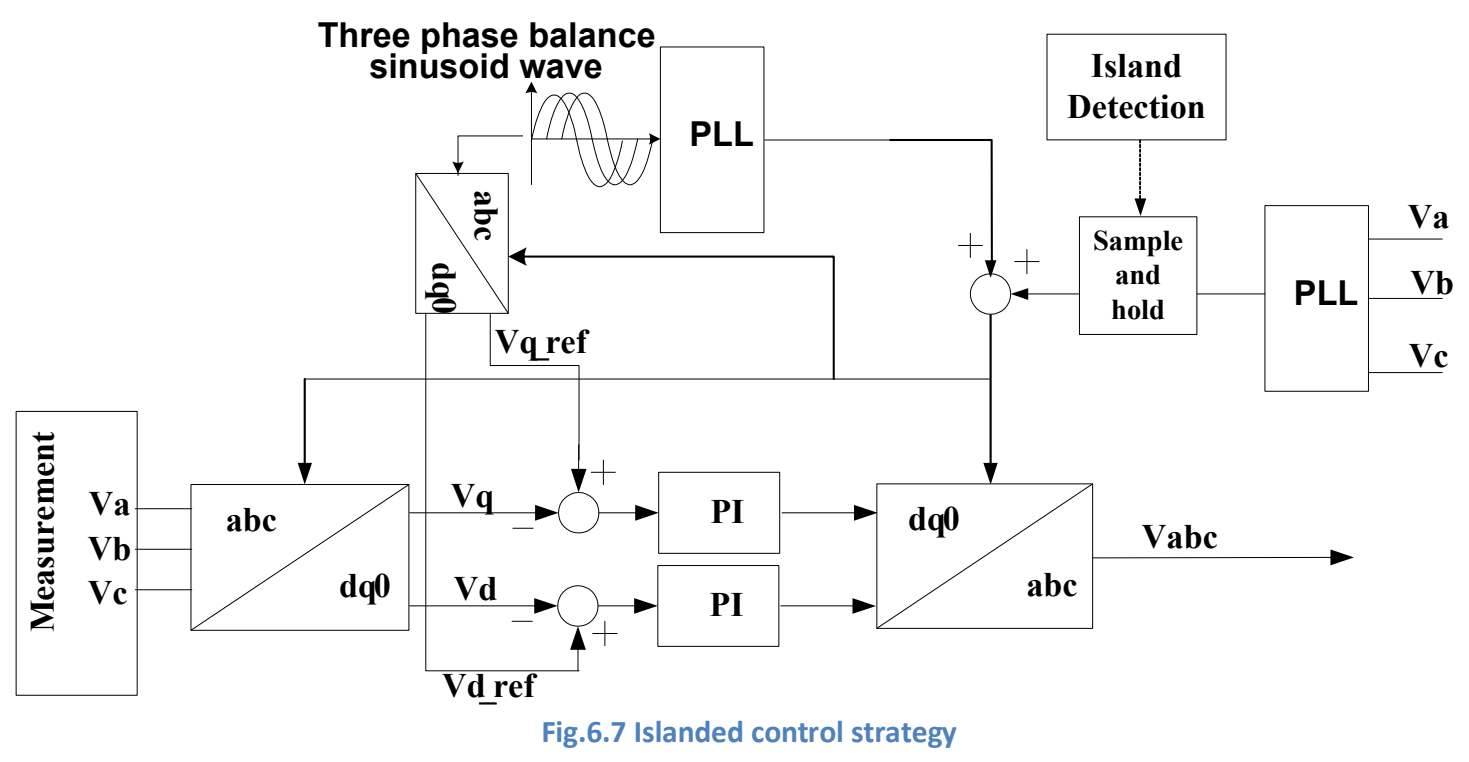

- $\quad$ Reactive power coordination

The variable reactive power output of wind farm is compensated by the dynamic reactive power of the battery. Thus the battery inverter is under voltage control mode in islanded operation.

- $\quad$ Active power coordination

In order for wind system to be operated in maximum power tracking mode, a battery is used as the governor of the wind turbine and is regulated as shown in Fig.6.6. The battery absorbs energy or injects energy to maintain the DC-link voltage of the wind converter. As a result, the wind farm is capable of provide the load. In case of large wind speed drop, load shedding operation will disconnect part of the load to restore system frequency.

\subsubsection{Battery operation control}


The State of Charge (SOC) of the battery is essential in the coordinated control. It is necessary to avoid depleting or overcharging the battery. Moreover, time deadband $t_{d b}$ prevents frequent operation mode changes and here $t_{b}$, the time duration, under charging/discharging modes should be greater than $t_{d b}$ of $0.033 \mathrm{~s}$..

$$
\text { BatteryMode }=\left\{\begin{array}{c}
\text { discharging, } S O C>S O C_{\text {low }} \& P_{\text {battset }} \text { or } Q_{\text {battset }}>0 \& t_{b} \geq t_{d b} \\
\text { charging, } S O C<S O C_{u p} \& P_{\text {battset }} \text { or } Q_{\text {battset }}<0 \& t_{b} \geq t_{d b}
\end{array}\right.
$$

where, $S O C_{\text {low }}$ and $S O C_{u p}$ are the lower and upper limits of $S O C, P_{\text {battset }}$ and $Q_{\text {battset }}$ are the reference active and reactive power output of battery. In order to increase the life span of battery storage without compromising the control objectives, a thorough charging/discharging control scheme is applied. The concept of this control strategy is to introduce a hysteresis band and add an idle mode in addition to the charging and discharging modes. In idle mode, battery is still connected to the system but it is not charging or discharging unless the idle mode has been deactivated. The idle mode is activated to protect battery life if one of the following criterions is met,

1) The reference power is within the deadband [-Pband, + Pband].

2) The reference power exceeds the deadband upper limit, and the battery SOC violates SOC_low.

3) The reference power violates the deadband lower limit, and the battery SOC violates SOC_up.

However, the charging mode is activated based on equation (6.12), until the battery SOC reaches its upper limits $S O C_{-}$up according to criterion 3. The battery may oscillate between charging and idle mode at this operation point. To avoid excessive charging or discharging frequently, the battery is charged fully unless discharging mode is activated.

\subsection{Simulation Study}

A comprehensive simulation study was carried out in PSCAD/EMTDC to verify the performance of the MPC based coordinated controller. A 2 MW direct drive wind turbine consisting of a PMSG and an AC-DC-AC converter is connected to the system through LR filter as shown in Fig.6.1. The PMSG has 84 poles with a base angular frequency of $117.93 \mathrm{rad} / \mathrm{s}$. The simulation time step is chosen as $0.0001 \mathrm{~s}$. A $0.5 \mathrm{MW}$ battery is also connected to the 0.48 $\mathrm{kV}$ PCC bus. The total load of the system is $1.5 \mathrm{MW}$. Several scenarios are simulated and the results are presented in this section. 
6.3.1 Comparison of MPC controller with industry standard
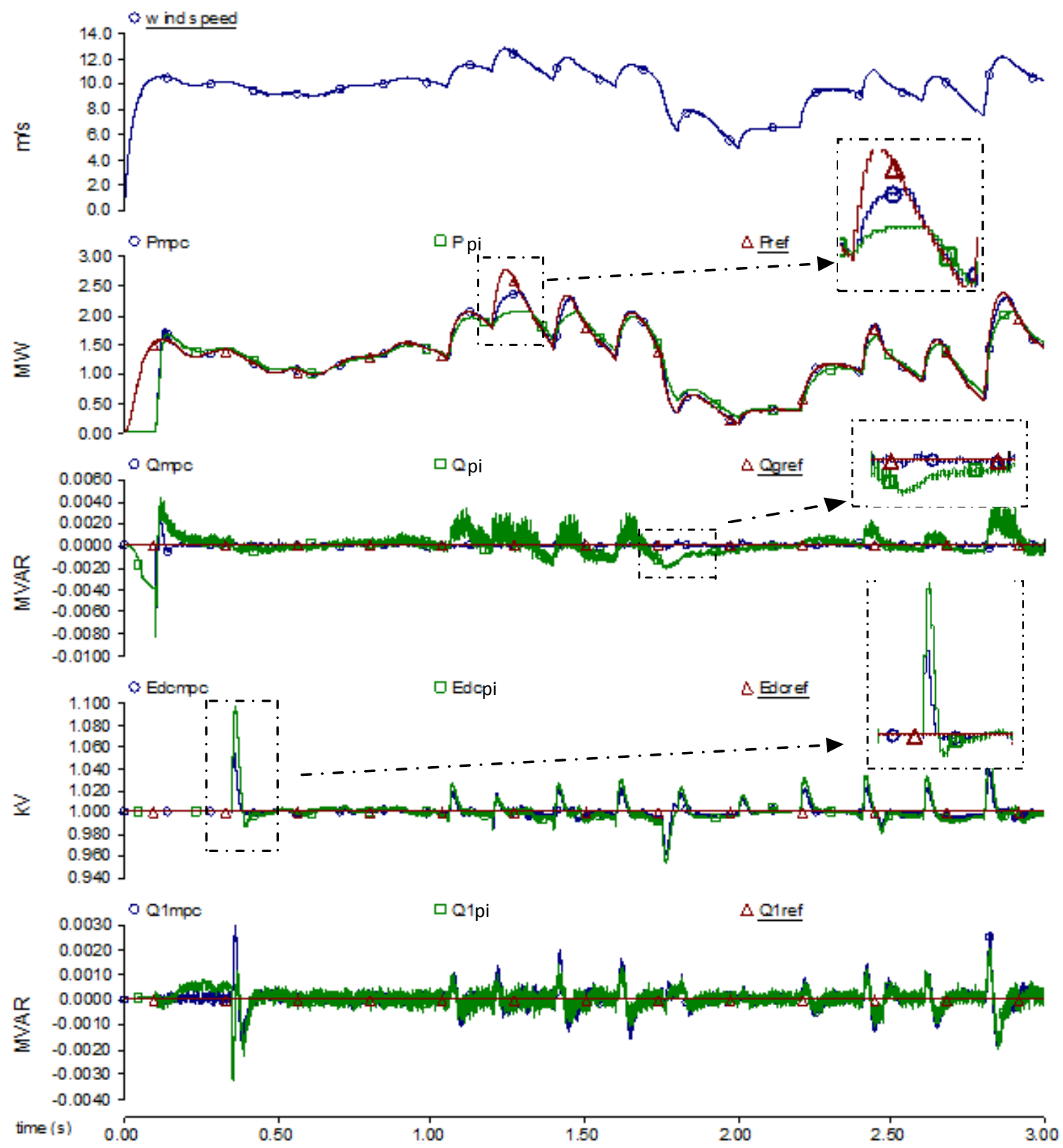

Fig.6.8 Comparison between MPC and optimized PI controller

The performance of MPC is compared with the PI controller with optimized parameters. The optimized PI parameters are obtained by the genetic algorithm proposed in section 5.6. An actual 3s wind speed profile is used as shown in Fig.6.8. $P$ and $Q$ are the active and reactive power of VSI-1, and $Q 1$ is the reactive power of VSI-2. The subscripts $m p c$, pi and ref denote MPC, PI controller and reference. Fig.6.8 shows that both the PI controller as well as MPC controller maintains 0 reactive power exchange between wind turbine and VSI-1 and between grid and VSI-2. It is also seen that the active power output of wind generator can 
track the wind speed variations with the proposed MPC and the optimal PI controller. The reactive power output and DC link voltage fluctuations are within \pm 0.001 Mvar for reactive power and \pm 0.02 p.u. for DC link voltage. The maximum overshoot of reactive power is less than 0.003 Mvar, and the overshoot of 1.09 p.u. in DC link voltage at $0.35 \mathrm{~s}$ is caused by the excessive wind speed. The rotor side converter controller maintains the active power output at $2 \mathrm{MW}$ when the wind speed exceeds the rated value. It can be seen from the figures that MPC has faster response, less overshoots and less steady state error in dc bus voltage, active and reactive power. The tracking error in Fig.6.8 is caused by the current limiter in wind converter to prevent the converter from over-loading. The rating of wind converter is $2 \mathrm{MW}$ in this study. The active power reference is obtained from equations (6.6) and (6.7), and the value can be higher than $2 \mathrm{MW}$ when the wind speed exceeds the rated value. Under such condition, the current of wind converter is limited by the current limiter to prevent the overloading of wind converter. Thus, the rotor side converter controller maintains the active power output at $2 \mathrm{MW}$ when the wind speed exceeds the rated value.

\subsubsection{Reactive power coordination under grid-connected operation}

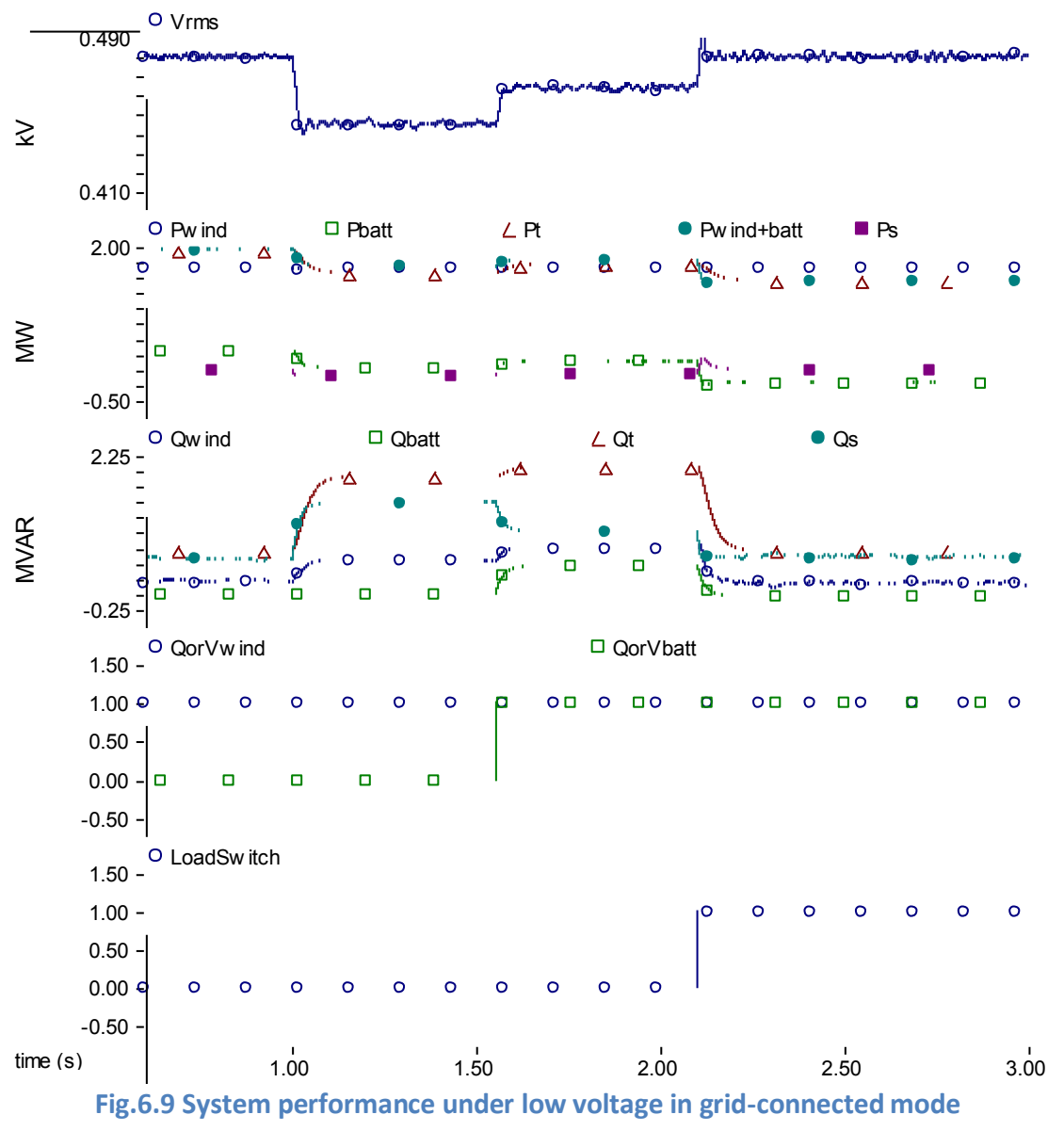


The four level protection capability of the proposed reactive power coordination under gridconnected operation is evaluated under severe voltage variations due to large inductive or capacitive load changes. Fig.6.9 plots the PCC bus voltage, real and reactive power, the operation mode (0: 0-RPC, 1: Voltage control) and the switch status of dynamic load (0: close, 1: open). The subscripts wind, batt, $t$, wind + batt, $s$ denote wind farm, battery, total load, wind and battery, and system, respectively. Initially the wind system is operating in wind dominant mode with Vrms as $0.48 \mathrm{kV}$, and the wind speed is maintained as constant. A 2 MVAR load step change at $1 \mathrm{~s}$ results in Vrms violating the lower voltage limit and an increase in reactive power output of wind turbine from 0.2 MVAR to its maximum capacity of 0.88 MVAR. The system transitions to wind and battery mode at $1.5 \mathrm{~s}$ which improves the voltage profile but cannot remove violations even though battery reaches its maximum reactive power output. The system transitions to shedding mode where the dynamic load is disconnected to restore Vrms to $0.48 \mathrm{kV}$.

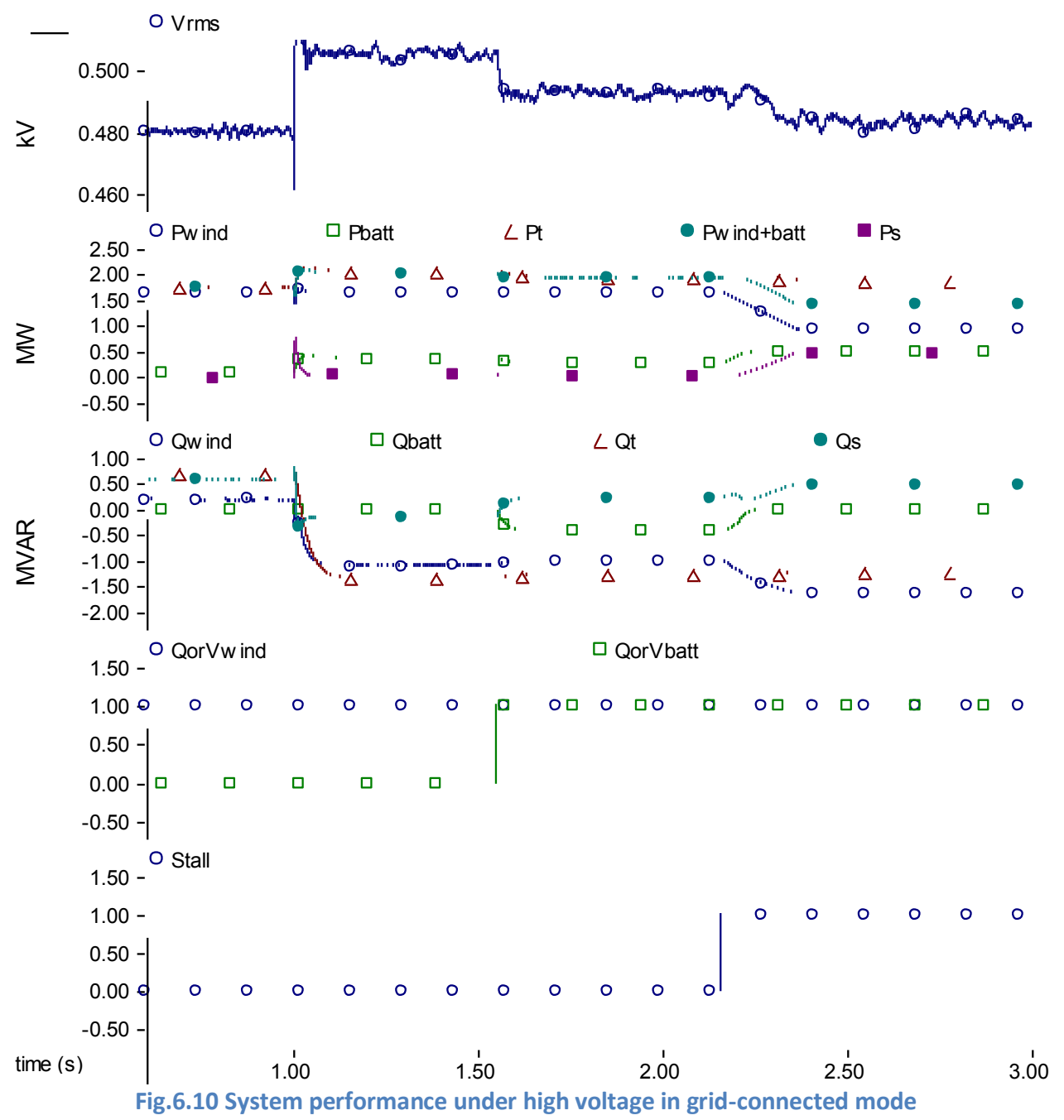


Similarly, when a load step at 1s results in Vrms violating higher voltage limit, the reactive power output of wind farm decrease to -1 MVAR as shown in Fig.6.10. Since the bus voltage is not within limit in the wind dominant mode even under wind farm generating maximum reactive power output, the system transitions to wind and battery mode at $1.5 \mathrm{~s}$ which improves voltage profile but cannot remove violations even though battery reaches its maximum reactive power output. The system transitions to stall operation mode at $2.2 \mathrm{~s}$, where the blade angle gradually increases which results in decrease in Pwind and increase in Qwind from -1 MVAR to -1.5 MVAR and restoring Vrms to $0.48 \mathrm{kV}$.

6.3.3 Active power coordination under grid-connected operation

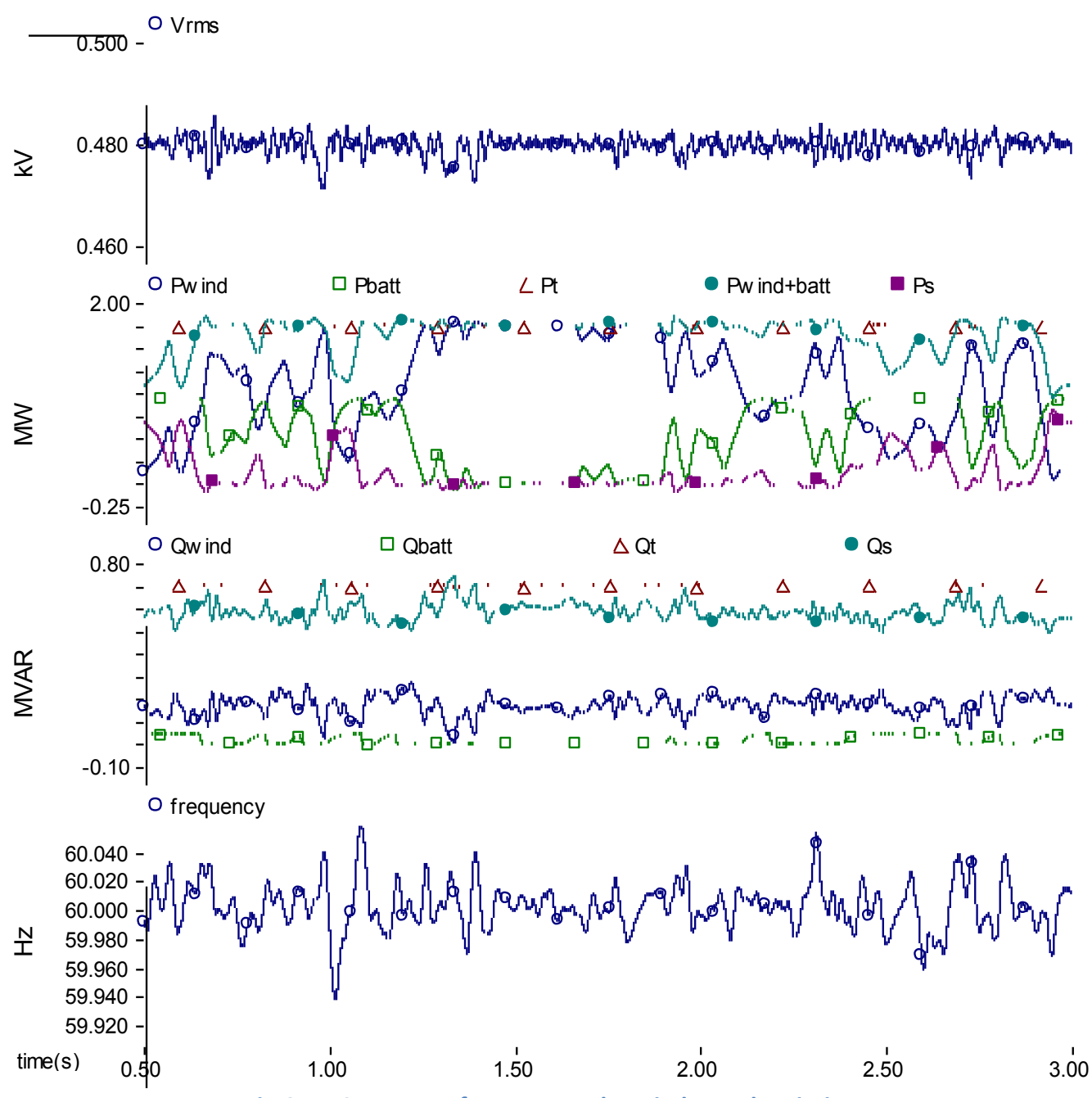

Fig.6.11 System performance under wind speed variation

The active power output of wind farm changes as shown in Fig.6.11 which changes in wind speed as shown in Fig.6.8. Wind and battery maintain power balance in the microgrid with no power exchange with the main power system. However, under low wind speed at $2 \mathrm{~s}$ the 
battery is at its maximum active power generation. The coordinated controller adjust the reactive power output of wind farm to maintain the PCC bus voltage at $0.48 \mathrm{kV}$, and the power mismatch is compensated by the main grid. The proposed coordinated control also provides active output power control that maintains system frequency.

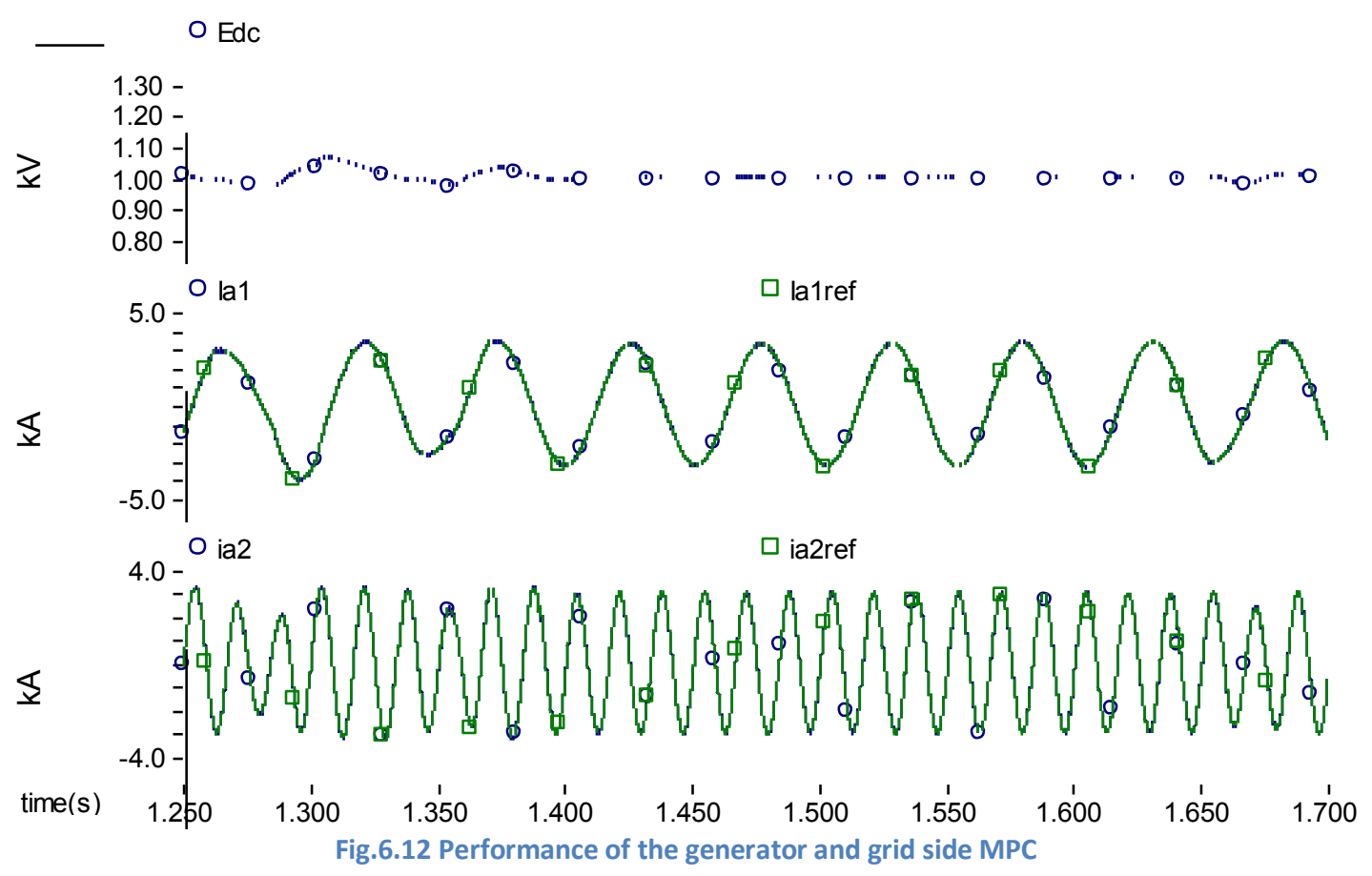

Fig.6.12 plots currents ia1 and ia2 of grid side and generator side MPC, respectively. It is seen that ia1 and ia2 track the reference current ia1ref and ia2ref while interconnecting the lowvarying frequency system on generator side with the $60 \mathrm{~Hz}$ electric power system.

6.3.4 Active and reactive power coordination under transition from grid-connected to islanded operation

The PCC bus voltage and wind converter output current when the microgrid is disconnected from main grid at 1.5s is as shown in Fig.6.13. There is no phase shift in the PCC bus voltage Va2 during the islanding. However, the current of VSI oscillates for one cycle.

As seen from Fig.6.14 the island power balance is disturbed when there is a large wind speed drop at 2.4s. Some of the dynamic load is disconnected at $2.6 \mathrm{~s}$ and battery adjusts reactive power output to compensate for voltage oscillation due to active power output variations of wind converter to maintain Vrms at $0.48 \mathrm{kV}$. The system frequency is maintained at $60 \mathrm{~Hz}$. 


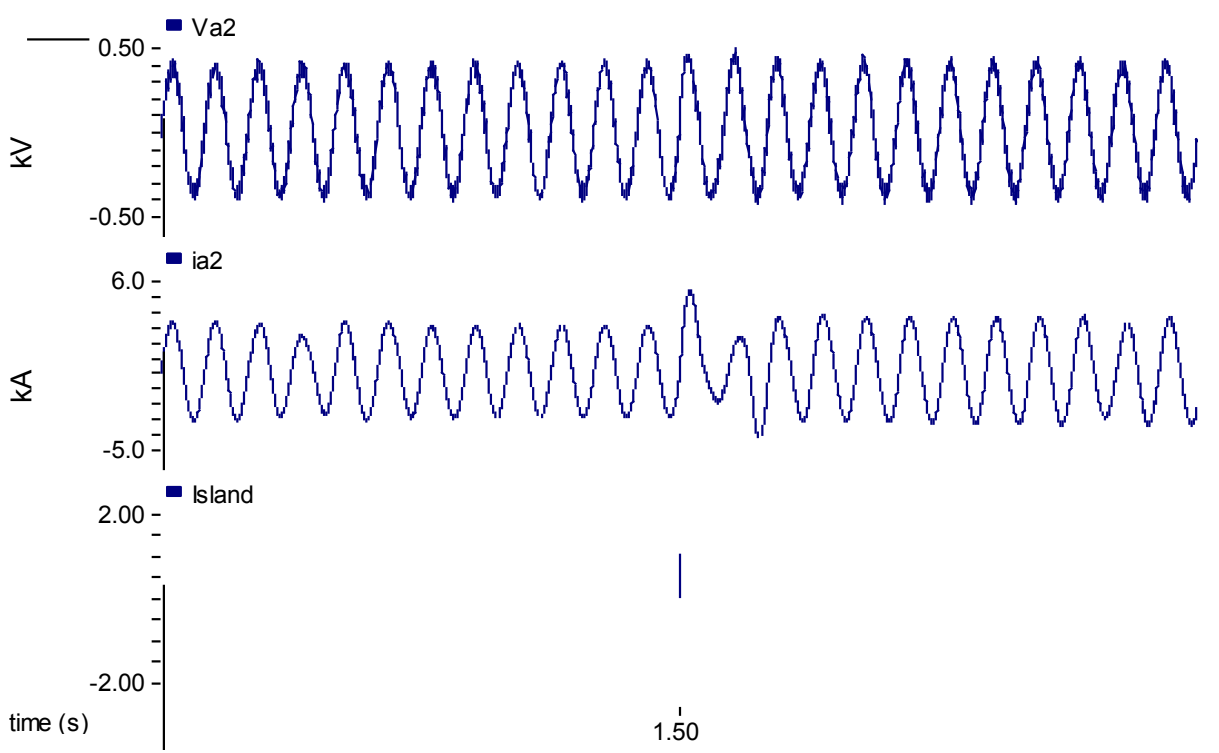

Fig.6.13 System response to operation mode change
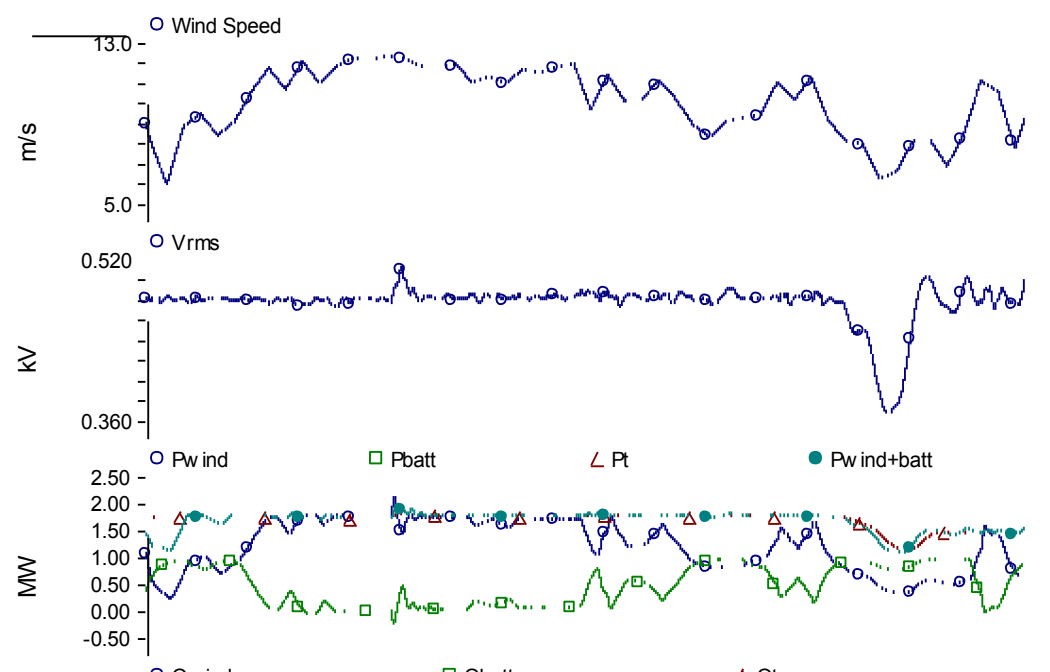

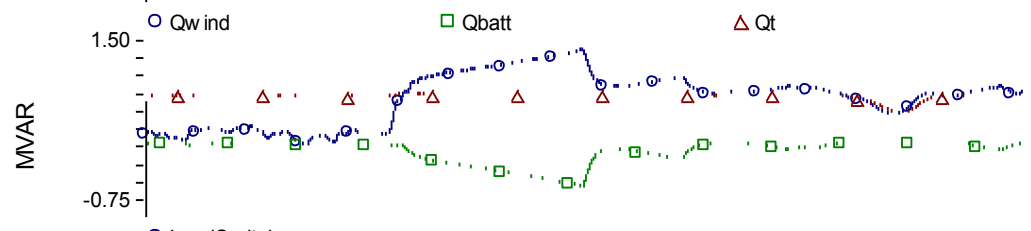

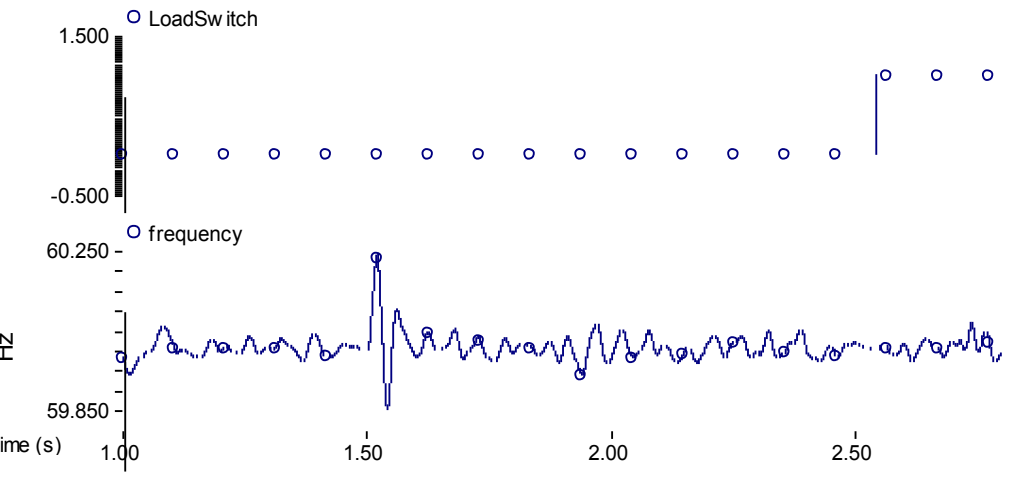

Fig.6.14 System performance during the transition from grid-connected to islanded mode 
6.3.5 Active and reactive power coordination under transition from islanded to gridconnected operation

The system performance from islanded to grid-connected operation is shown in Fig.6.15 and Fig.6.16. As seen from the figures, there is no phase shift in the PCC bus voltage during the transition from islanded to grid-connected mode. The microgrid is able to provide the local load before and after the transition, and the PCC bus voltage is maintained at $0.48 \mathrm{kV}$.

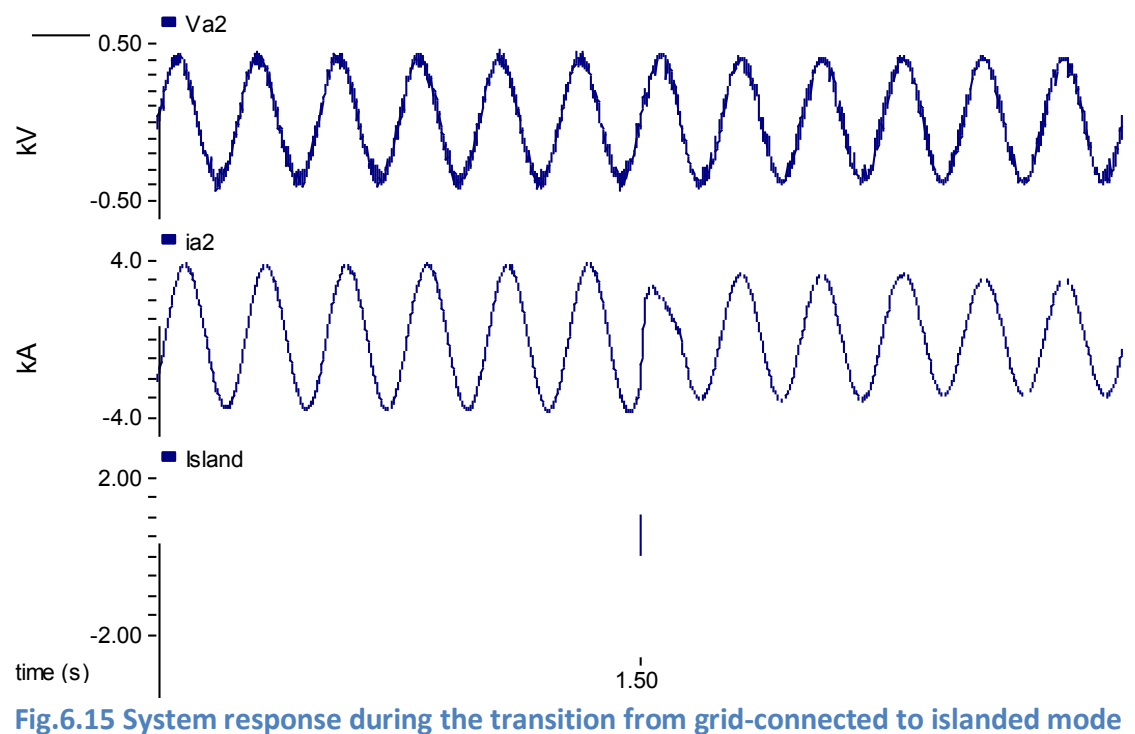

\subsubsection{Reactive and active power coordination under black start in islanded operation}

Under black start, the average wind energy calculated by wind forecasting combine with the maximum battery capacity determines the load that can be supplied. Fig.6.17 shows the system performance under black start with 1.2 MW load connected. Wind farm is connected at $0.3 \mathrm{~s}$ and battery is connected $0.1 \mathrm{~s}$ after. It is seen that the wind farm reaches steady state at $0.6 \mathrm{~s}$, and the PCC bus voltage Vrms is maintained at $0.48 \mathrm{kV}$. 


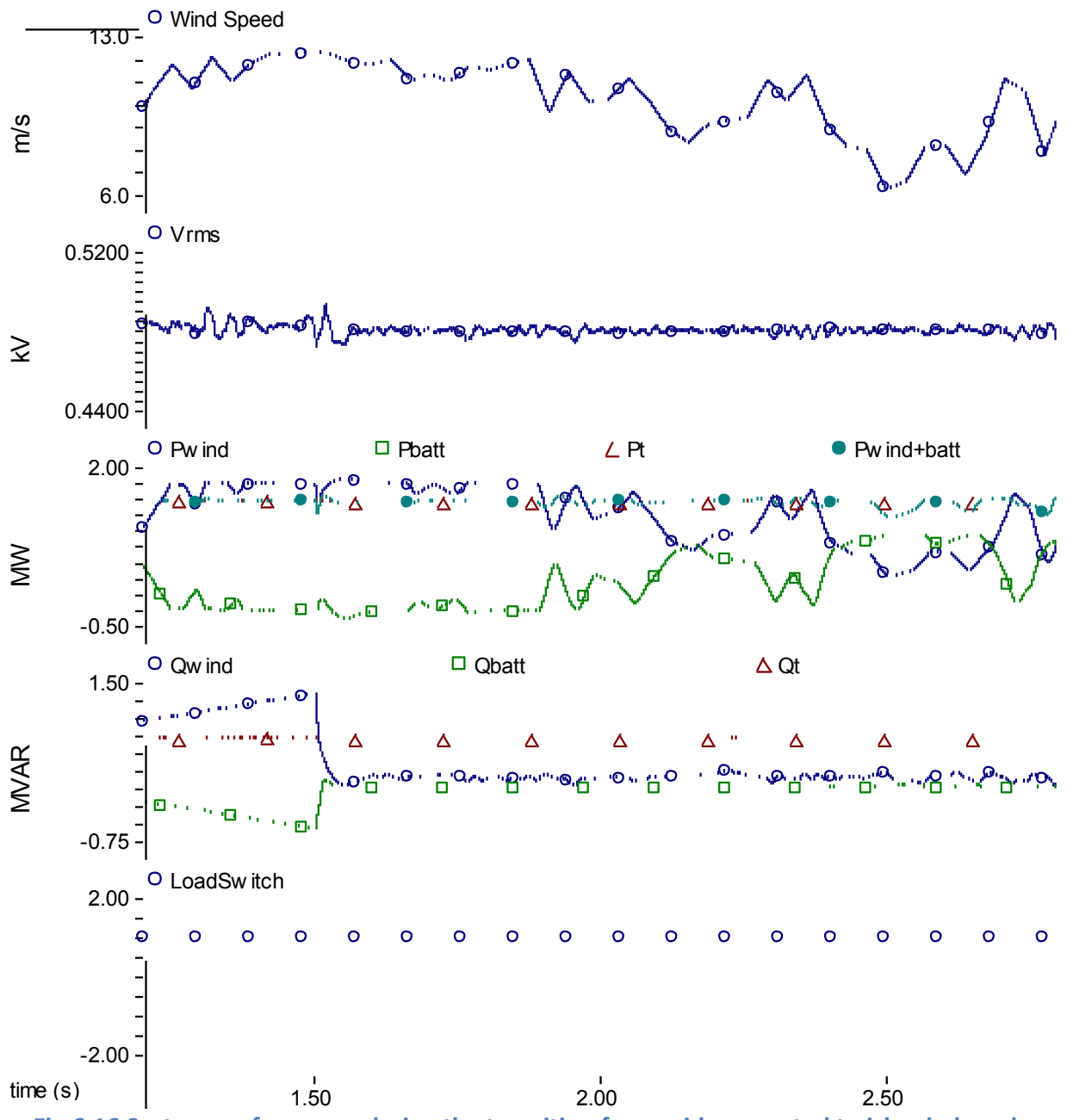

Fig.6.16 System performance during the transition from grid-connected to islanded mode 

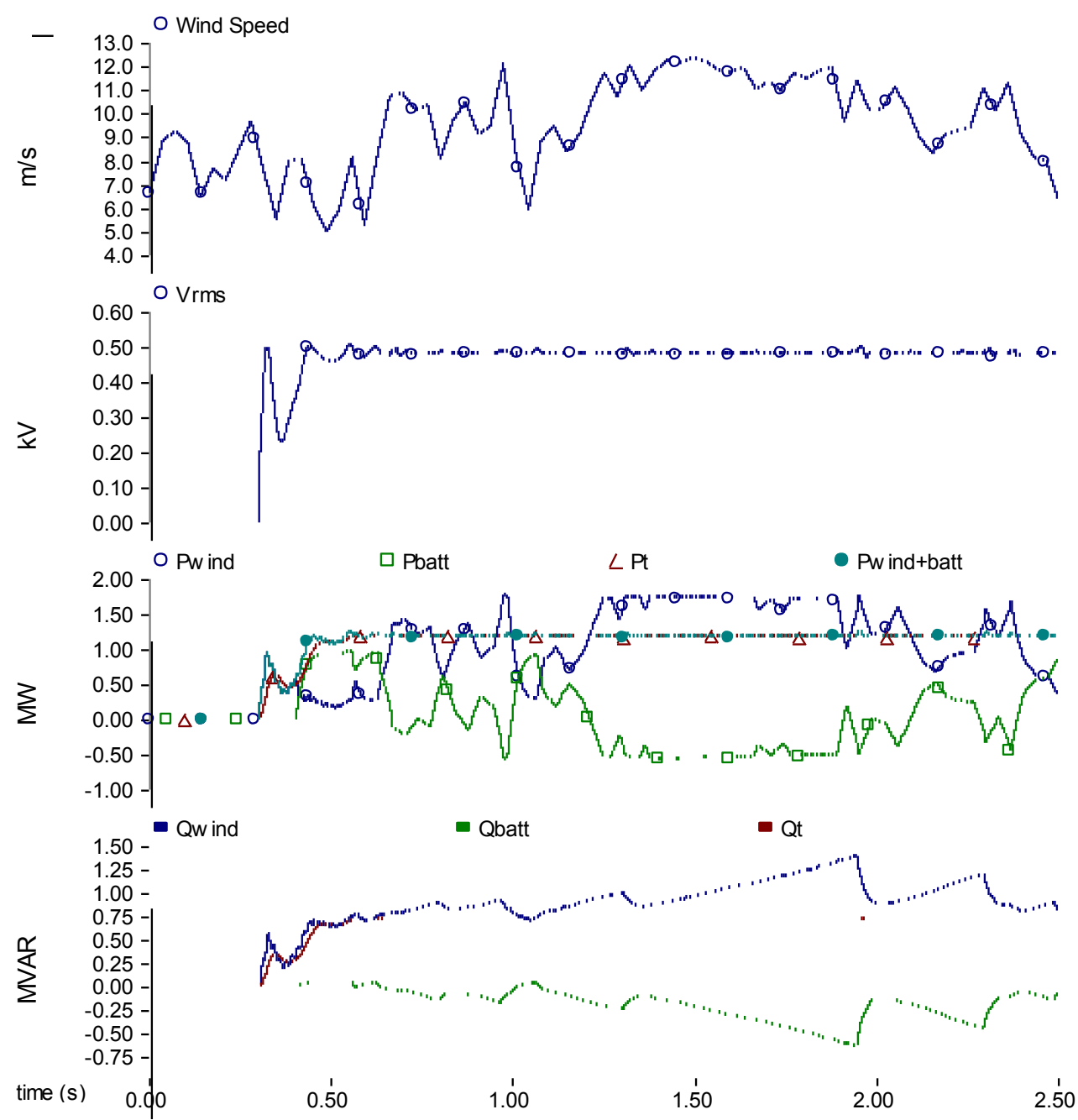

Fig.6.17 System performance during black start in islanded operation

\subsection{Chapter Conclusion}

This section presents a MPC based supervisory control system that coordinates the operation of wind farm and battery storage system in a microgrid for grid-connected and islanded operation. The MPC increases the accuracy of maximum wind energy capture as well as minimizes the power oscillations caused by varying wind speed. The proposed coordinated controller mitigates both active and reactive power disturbances that are caused by the intermittencies in wind speed and load change. Thus the control strategy adds an extra degree of flexibility to the microgrid by providing four level of protection, and maintaining power balance. 


\section{Chapter 7. Adaptive Critic Design Based Dynamic Stochastic Optimal Control Design for a Microgrid with Multiple Renewable Resources}

This section extends the work of [109] by implementing the System Wide Adaptive Predictive Supervisory Control (SWAPSC) scheme. The proposed SWAPSC consists of a DHP based system layer control, a coordinated control based local layer control, and a Model Predictive Control based device layer control. The SWAPSC smoothens the PV and wind generation output, provides dynamic reactive power support, and reduces the power loss as well as maximizes the usage of battery storage. Only grid-connected operation of the microgrid is considered in this study since the aim of this study is to test the effectiveness of the proposed scheme. Further study related to island operation will be presented in future work.

\subsection{Overall Frame of the Proposed SWAPSC}

The IEEE 13 node feeder is used as a platform system for evaluating the performance of the proposed controller. Fig.7.1 hows the single-line diagram of the IEEE 13 node system that includes a direct drive wind farm, a PV system, and two battery systems. The system is unbalanced with voltage at node 634 as $0.48 \mathrm{kV}$ and all other nodes as $4.16 \mathrm{kV}$ with total load of 4.053 MVA and lagging power factor of 0.855 and a regulator at node 650. A PV set consists of a PV farm and battery 1 is interconnected at node 680 through a $0.48 \mathrm{kV} / 4.16 \mathrm{kV}$ transformer, and a wind set consists of a wind power generation system and battery 2 are interfaced at 634 directly. Each battery system is equipped with an inverter-based power electronic interface. PV, wind and battery systems are modeled in detail, with the power electronic switch dynamics taken into account. 


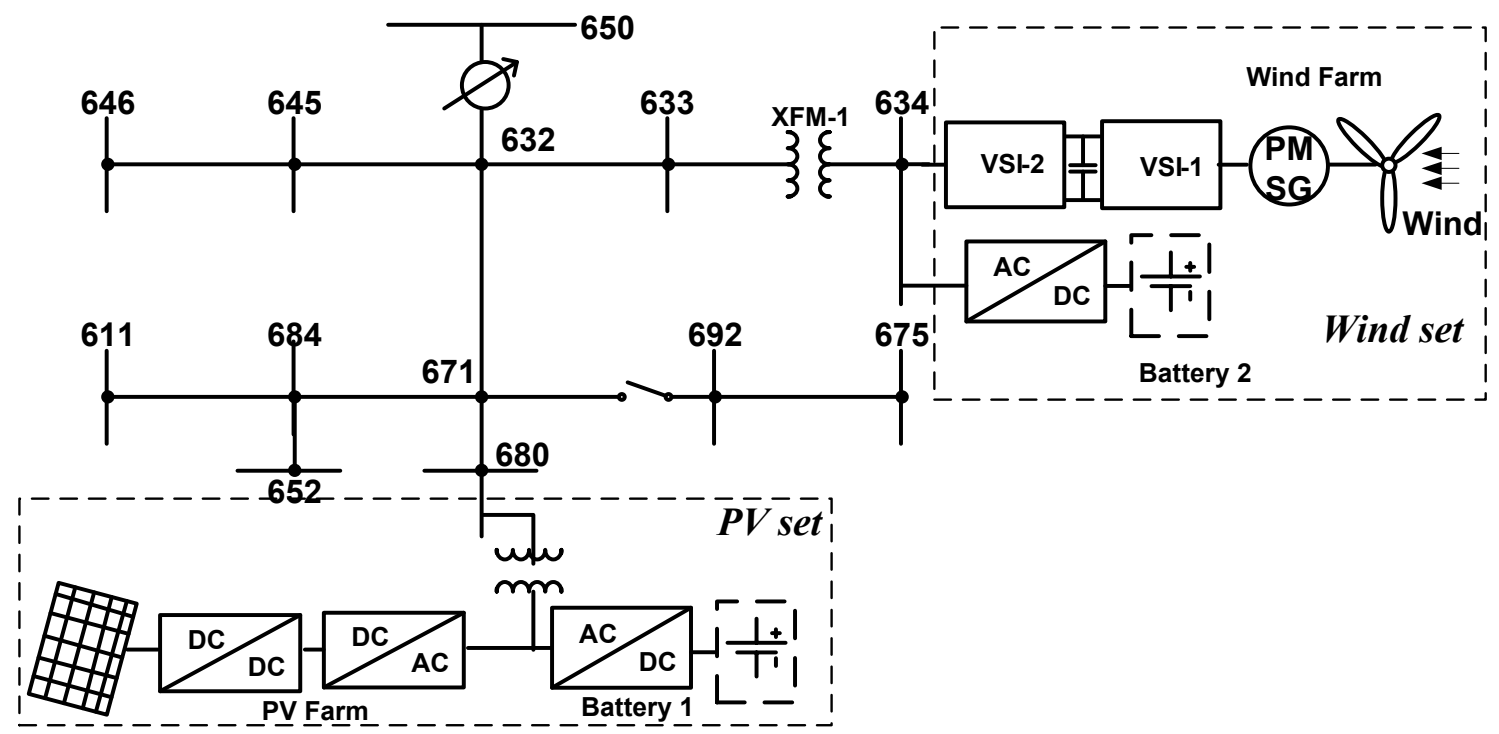

Fig.7.1 IEEE 13 node system with renewable resources.

It is essential to optimally operate the power system for which the dynamic adjustment of real and reactive power of renewable generators is needed. Hence, the principle roles of a well designed microgrid controller are:

- $\quad$ Active power dispatch of PV and wind set ;

- Voltage regulation at PCC buses;

- $\quad$ SOC management;

- Overall power loss reduction;

- $\quad$ Control effort minimization;

Improved life spans of batteries, maintaining maximum reactive power reserve as well as increased control accuracy are also important in practical applications. Layered control structure can address these requirements at different layers since they are of different significances and time scales [110]. A SWAPSC scheme is as shown in Fig.7.2 , consisting of three layers, namely system layer, local layer and device layer. The DHP based system layer collects online system-wide power system quantities, simulates the critical thinking of human intelligence based on neural network computations, and determines a sequence of optimal dispatch signals $\left[P_{\text {pvsetref }}, Q_{\text {pvsetref }}, P_{\text {windsetref }}, Q_{\text {windsetref }}\right]$ for PV and wind sets, by learning of the control behavior patterns. Here, $P_{\text {pvsetref }}$ and $Q_{p v s e t r e f}$ are the reference of total injected active and reactive power for PV set, and $P_{\text {windsetref }}$ and $Q_{\text {windsetref }}$ are the references of total injected active and reactive power for wind set. These dispatch signals ensure the active power of PV and wind are dispatchable, and help maintain the 
interconnecting PCC buses voltages within limit, maintain the SOC of both batteries, and reduce overall power loss and minimize control effort. The insolation level, wind speed, and the operation state of both batteries are not considered in the calculation of references which can compromise the battery life and reactive power reserves.

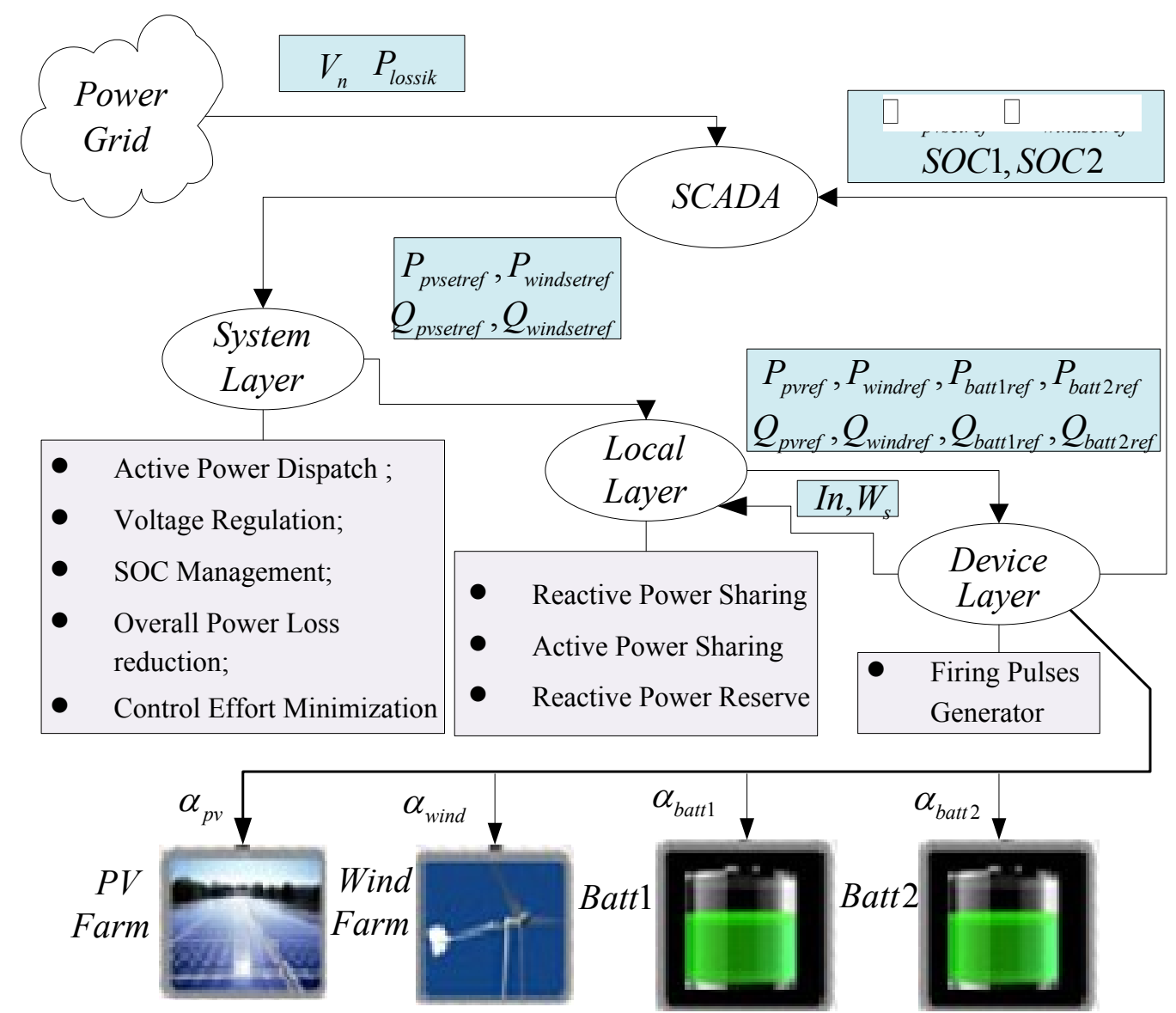

Fig.7.2 Structure of the proposed SWAPSC. $V_{n}$ is the voltage of bus n. $P_{\text {lossjk }}$ is the loss of line $j$ to $k$. In is the insolation level. $W_{n}$ is the wind speed. $\alpha$ is the firing pulse vector. Subscripts $p v$, wind, batt1 and batt2 denote PV farm, wind farm, battery system 1 and battery system 2, respectively.

The local layers dispatch signals have knowledge of the current operation status of PV, wind farm, battery storage and power grid. There are two local layers as shown in Fig.7.2, determine the operating mode of PV and Wind as well as the reference active and reactive power of battery from the reference dispatch signals of the system layer to minimize battery capacity without compromising the overall controller performance. The device layer determines the firing pulses ( $\alpha_{p v}$ for PV inverter, $\alpha_{\text {wind }}$ for wind converter, $\alpha_{\text {batt } 1}$ for battery 1 inverter, and $\alpha_{\text {batt2 }}$ for battery 2 inverter) of the power electronic devices according to the operation mode determined by local layer control. MPC provides current waveform 
tracking to achieve the desired reference active and reactive power. The detailed design of each control layer is presented in the following sections.

\subsection{DHP Based System Layer Control Design}

The optimal operation point varies with different control objectives. Therefore, the key to the design of the SWAPSC's system control layer is to find the relationship between dispatch commands and the objectives. However, the inherent relationships can be very complicated and are not generic, relying on specific control complexity and objectives, in particular for distribution systems [111]. The optimization problem is formulated as in (7.1),

$$
\begin{gathered}
\min E=\sum_{n=1}^{\infty} F\left(y_{1}(n+1), y_{2}(n+1), \ldots, y_{m}(n+1)\right) \\
\text { s.t. } y(n+1)=f(y(n), A(n)) \\
Y_{\min } \leq Y(n) \leq Y_{\max }
\end{gathered}
$$

The objective is to determine a series of control actions $[A(n) \mid 1 \leq n \leq \infty]$ that can minimize the error $\mathrm{E}$ to track reference signals. The error $\mathrm{E}$ at time constant $n$ is a function of outputs $y(n+1)=\left[y_{1}(n+1), y_{2}(n+1), \ldots, y_{m 2}(n+1)\right]$ at time constant $n+1$ where $y_{1}(n+1)$, $y_{2}(n+1), \ldots, y_{m 2}(n+1)$ are case-dependent and can be a combination of power system quantities such as active power, bus voltage, or system loss and the outputs at $n+1$ are dependent on the output at previous time instant $y(n+1)$ and the control action $A(n)$. $G(y(n+1)$ is a function of current and future states of the power system which are not known. Hence, there are three issues that need to be addressed for this, first, the system behavior, i.e., the relationship between the control command and the corresponding system responses at next time step need to be modeled. It is known that the mathematical representation of a power system with detailed renewable generator models often involves higher order differential equations. Thus, an accurate and efficient system representation is very complex and almost impossible to achieve. The nature of the online optimization does not allow the control actions to be reversed once implemented [111]. Thus, the controller should be able to learn ahead the optimality of future control actions and should be adaptive to the frequent operation variations of renewable resources. 


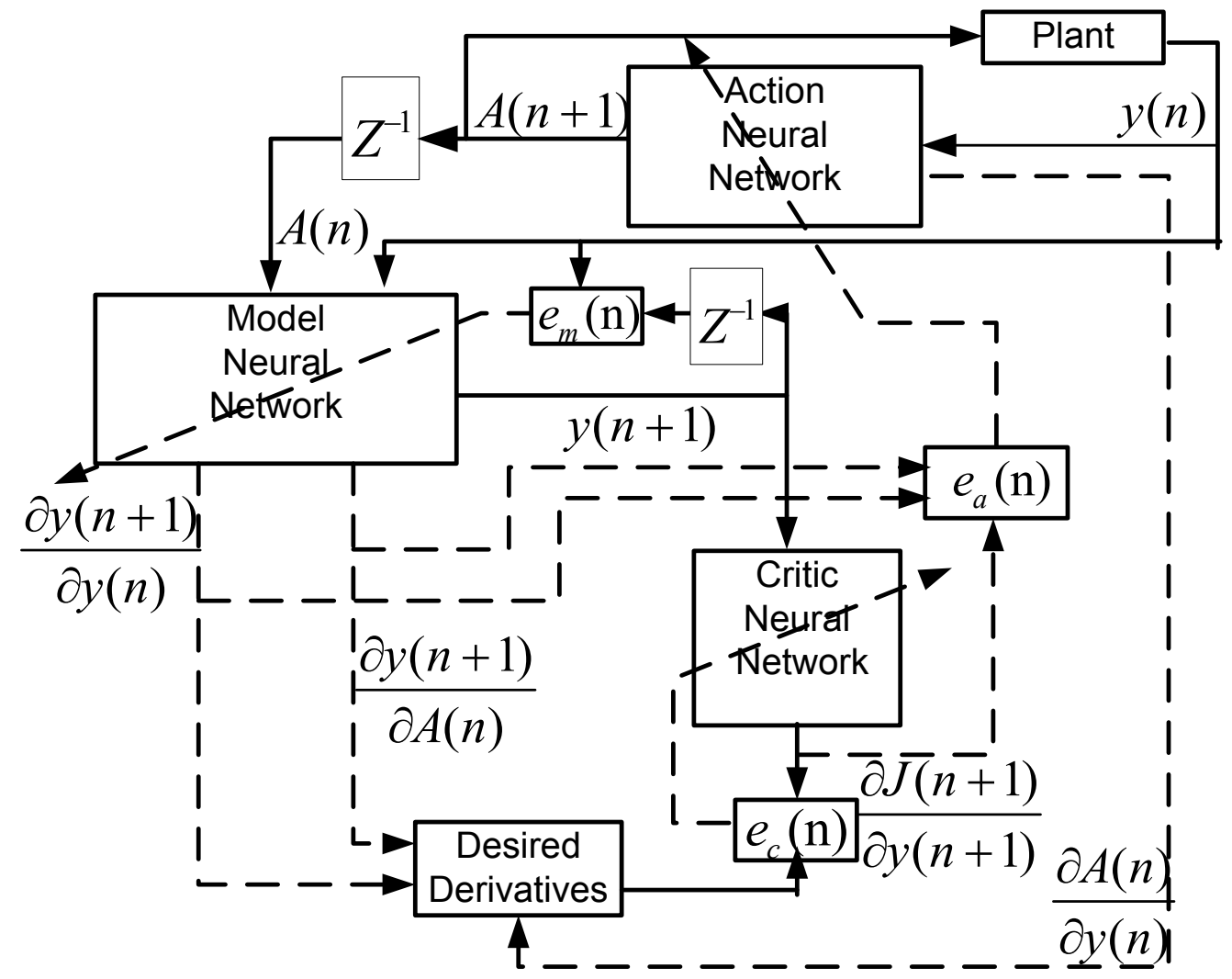

Fig.7.3 System layer control using DHP

The DHP algorithm as shown in Fig.7.3 provides the solution to the aforementioned issues by using three neural networks, namely model network, action network, and critic network. The model network identifies the non-linear relation between control action and system response, and provides an accurate model to represent the system dynamics. The control action $A(n)$ and system response $Y(n)$ are the input vectors of the model network and are based on the error $e_{m}(n)$ between actual and reference output. The model network is trained to predict the system response vector $Y(n+1)$ at time $n+1$. The outputs from model network are the partial derivatives of $Y(n+1)$ with respect to $A(n)$ and $Y(n)$ which train the critic and action networks. The critic network learns to approximate the strategic utility function or cost-togo function $J(n)$ in a step by step manner by solving the Bellman equations as shown in (7.2), where

$$
J(n)=\sum_{i=0}^{\infty} \gamma^{i} \cdot U(n+i)=U(n)+\gamma \cdot J(n+1)
$$

the discount factor is $0<\gamma<1$, and $\mathrm{U}(\mathrm{n})$ is a user defined utility function that represents the performance metric of the system at time instance $n$ which is a function of control vector 
$A(n)$ and output vector $Y(n)$. It is seen from (7.1) and (7.2) that the optimal control strategy of the system layer is to minimize $\mathrm{J}(\mathrm{n})$, in a closed-loop control under complex system constraints. Thus, at any instant, if we can find an action $A(n)$ that optimizes the short term $\mathrm{J}(\mathrm{n})$, an optimized $U(n)$ over all future times is ensured. The inputs to the critic network are the predicted system response vector $Y(n+1)$, and the critic error vector $e_{c}(n)$ (obtained from back propagated derivative signals from model and action networks) and are used for critic network training. The critic network estimates the gradient of $J(n+1)$ with respect to $Y(n+1)$ and together with the output directives from model network generates an action error vector $e_{a}(n)$ for action network training. The action network considers the system state $Y(n)$ at time $n$ and determines the control action $A(n+1)$ which aims to minimize $J(n+1)$ obtained from critic network. The derivative of control action $A(n)$ with respect to $Y(n)$ determines the critic error. The continuous optimal operation is realized in such a way that, at each calculation time step, the system control layer adjusts the reference active and reactive power of the PV and wind set. Details on implementation of DHP are introduced in this section.

\subsubsection{Control Objectives}

As discussed above, the cost-to-go function is essential in online optimization and is directly dependent on $U(n)$, the choice of which depends on the control and optimization objectives. The goals of optimization are to reduce the impact of intermittencies in PV and wind energy as well as improve the voltage stability and reduce line losses while minimizing control effort. The utility function $U(n)$ is defined in (7.3) and that includes dispatching error $U_{\text {disp }}(n)$, voltage deviations $U_{v o l}(n)$, state of charge $U_{\text {soc }}(n)$, line losses $U_{\text {loss }}(n)$ and the control effort $\mathrm{U}_{\text {con }}(\mathrm{n})$ prioritized by, $\mathrm{k}_{\mathrm{disp}}, \mathrm{k}_{\mathrm{vol}}, \mathrm{k}_{\mathrm{soc}}, \mathrm{k}_{\text {loss }}$ and $\mathrm{k}_{\mathrm{con}}$ are the corresponding weight factors whose selection depend on the priority of the corresponding component in $U(n)$.

$$
U(n)=k_{\text {disp }} U_{\text {disp }}(n)+k_{\text {vol }} U_{v o l}(n)+k_{\text {soc }} U_{\text {soc }}(n)+k_{\text {loss }} U_{\text {loss }}(n)+k_{\text {con }} U_{c o n}(n)
$$

The dispatch error $U_{\text {disp }}(n)$ is calculated using (7.4) and is representation of the impacts of present action on the dispatch error of PV and wind sets, calculated using $\mathrm{P}_{\text {pvset }}$ act $(n)$ and $\mathrm{P}_{\text {windset }_{\text {act }}}(\mathrm{n})$, the actual active power outputs of PV and wind set, respectively.

$$
\begin{aligned}
U_{\text {disp }}(n) & =\Delta P_{\text {pvset }}(n)^{2}+\Delta P_{\text {windset }}(n)^{2} \\
& =\left(P_{\text {pvset }_{\text {ref }}}(n)-P_{\text {pvset }_{\text {act }}}(n)\right)^{2}+\left(P_{\text {windset }_{\text {ref }}}(n)-P_{\text {windset }_{\text {act }}}(n)\right)^{2}
\end{aligned}
$$


The optimal use of battery for smoothing the intermittent power output of PV and wind is to ensure the PV and wind set active power outputs are dispatchable on an hourly basis $[112,113,114]$. However, here, due to the complexity of the system model that includes DHP controller and the detailed PV, wind and battery systems, the duration of the simulation study is $5 \mathrm{~s}$ and the dispatch interval is $100 \mathrm{~ms}$. It is assumed that the $5 \mathrm{~s}$ ahead wind speed and insolation is accurate and available, a good choice of the desired active power dispatch set point for the PV and wind sets at the time step $n, P_{\text {pvset }}(n)$ and $P_{\text {windset }}(n)$ are the 100 ms average of the estimated active power output. Large dispatch interval (one hour) can be employed with simplified renewable resources models.

The effect of control action on voltage stability is defined as $U_{v o l}(n)$ and the voltages at interconnection nodes 634 and $680, \Delta \mathrm{V}_{634}(\mathrm{n})$ and $\Delta \mathrm{V}_{680}(\mathrm{n})$ are considered.

$$
U_{v o l}(n)=\left|\Delta V_{634}(n)\right|^{2}+\left|\Delta V_{680}(n)\right|^{2}
$$

The state of charge $U_{\text {soc }}(n)$ is calculated using (7.6) and here SOCs of battery systems 1 and 2, SOC1(n) and SOC2(n) are considered. $\mathrm{SOC}_{\mathrm{UL}}$ and $\mathrm{SOC}_{\mathrm{LL}}$ are the upper and lower limits of SOC, respectively. $U_{s o c}(n)$ increases dramatically whenever either of the SOC is out of bound, otherwise, remain negligible.

$$
U_{S o c}(n)=e^{\left(\left(\frac{\operatorname{SOC} 1(n)}{\operatorname{SOC}_{U L}}\right)^{4}-1\right)}+e^{\left(\left(\frac{\operatorname{SOC}_{2}(n)}{\operatorname{SOC}_{U L}}\right)^{4}-1\right)}+e^{\left(\left(\frac{\operatorname{SOC}_{L L}}{\operatorname{SOC1}(n)}\right)^{4}-1\right)}+e^{\left(\left(\frac{\operatorname{SOC}_{L L}}{\operatorname{SOC} 2(n)}\right)^{4}-1\right)}
$$

The line losses index $U_{\text {loss }}(n)$ and control effort $U_{\text {con }}(n)$ are calculated using (7.7) and (7.8),

$$
\begin{gathered}
U_{\text {loss }}(n)=P^{2}{ }_{\text {loss }}(n) \\
U_{\text {con }}(n)=P^{2}{ }_{\text {pvbatt }}(n)+(n)+P^{2}{ }_{\text {windbatt }_{r e f}}(n)+Q^{2}{ }_{\text {pvbatt }}(n)+Q^{2}{ }_{\text {windbatt }_{r e f}}(n)
\end{gathered}
$$

The solution obtained for this multi-objectives optimization problem using the critic, model and action networks is suboptimal. Recurrent Neural Networks (RNNs) proposed in [93] are implemented to construct critic, model and action networks. To be convenient, subscript c, m, and a denote critic, model and action network, respectively.

\subsubsection{Critic Network Design}

As mentioned in section 2.6, in DHP, the derivatives of the cost-to-go J[y(n+1)]with respect to $y(n+1)$ are approximated instead of $J[y(n+1)]$ using the critic network. Denote $\hat{\lambda}(n)=$ 
$\frac{\mathrm{d}(\mathrm{J}[\mathrm{y}(\mathrm{n})])}{\mathrm{dy}(\mathrm{n})}$ and $\hat{\mathrm{x}}(\mathrm{n}+1)=\frac{\partial(\mathrm{J}[\mathrm{y}(\mathrm{n}+1)])}{\partial \mathrm{y}(\mathrm{n}+1)}$ as the outputs of critic network at times $\mathrm{n}$ and $\mathrm{n}+1$. This approximation can only be accurate if the error defined in (9) is minimized. [93],

$$
E_{c}(n)=\left\|e_{c}(n)\right\|^{2}
$$

where

$$
\begin{gathered}
e_{c}(n)=\frac{d(J[y(n)]-\gamma \cdot J[y(n+1)]-U(n))}{d y(n)} \\
\frac{\partial(\mathrm{J}[\mathrm{y}(\mathrm{n}+1)])}{\partial \mathrm{y}(\mathrm{n})}=\hat{\lambda}(\mathrm{n}+1) \cdot\left(\frac{\partial y(n+1)}{\partial y(n)}+\frac{\partial y(n+1)}{\partial A(n)} \cdot \frac{\partial A(n)}{\partial y(n)}\right)
\end{gathered}
$$

and

$$
\frac{\partial U(n)}{\partial y(n)}=\frac{\partial(U(n))}{\partial y(n)}+\frac{\partial U(n)}{\partial A(n)} \cdot \frac{\partial A(n)}{\partial y(n)}
$$

Substitute (7.11) and (7.12) into (7.10) we obtain (7.13),

$$
\begin{aligned}
e_{c}(n)= & \hat{\lambda}(n)-\left\{\frac{\partial(U(n))}{\partial y(n)}+\frac{\partial U(n)}{\partial A(n)} \cdot \frac{\partial A(n)}{\partial y(n)}\right. \\
& \left.+\widehat{\gamma \cdot \lambda}(n+1)\left[\frac{\partial y(n+1)}{\partial y(n)}+\frac{\partial y(n+1)}{\partial A(n)} \cdot \frac{\partial A(n)}{\partial y(n)}\right]\right\}
\end{aligned}
$$

The derivatives in (7.13) are obtained from model and action networks as shown Fig.7.3. Fig.7.4 shows the structure of the RNN-type critic neural network. 


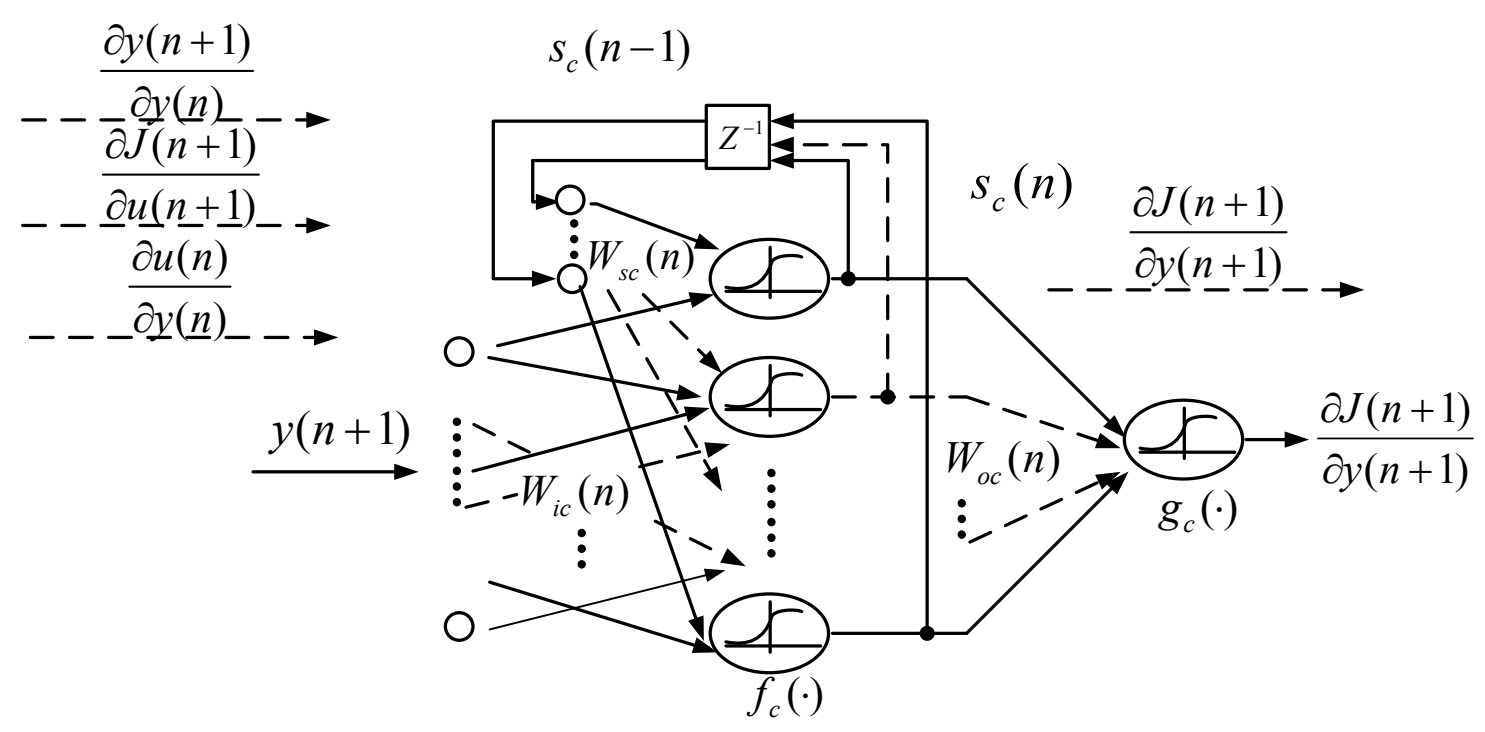

Fig.7.4 The structure of critic network

It is seen that the critic network consists of three layers: an input layer with input vector $y(n+1)=\left[\Delta P_{\text {pvset }}(n), \Delta P_{\text {windset }}(n), \Delta V_{634}(n), \Delta V_{680}(n), \operatorname{SOC} 1(n), \operatorname{SOC} 2(n), P_{\text {loss }}(n) \quad, \quad\right.$ a hidden layer with 20 neurons and an output layer with output vector $\hat{\lambda}(n+1)$. Here, $s(\cdot)$ is the internal state vector, $\mathrm{f}(\cdot)$ and $\mathrm{g}(\cdot)$ are the activation functions of hidden and output layer, respectively. The output of critic network is a nonlinear weighted sum of system responses and control actions as shown in (7.14),

$$
\hat{\imath}(n+1)=g_{c}\left(W_{o c} \cdot f_{c}\left[W_{i c} \cdot y(n+1)+W_{s c} \cdot s_{c}(n-1)\right]\right)
$$

Here the weights in output layer $\mathrm{W}_{\mathrm{oc}}$, in input layer $\mathrm{W}_{\mathrm{ic}}$ and in hidden layer $\mathrm{W}_{\mathrm{sc}}$ are updated to minimize error in (7.9). A commonly used update procedure, namely the backpropagation method based method as shown in (7.15)-(7.17) is used,

$$
\begin{aligned}
& W_{o}(n+1)=W_{o}(n)-\eta_{o} \frac{\partial E(n)}{\partial W_{o}(n)} \\
& W_{s}(n+1)=W_{s}(n)-\eta_{s} \frac{\partial E(n)}{\partial W_{s}(n)} \\
& W_{i}(n+1)=W_{i}(n)-\eta_{i} \frac{\partial E(n)}{\partial W_{i}(n)}
\end{aligned}
$$

The learning rates $\eta_{0}, \eta_{s}$, and $\eta_{i}$ control the update step size of corresponding weight matrices, and the error gradients for critic network are given by (7.18)-(7.20), 


$$
\begin{array}{r}
\frac{\partial E_{c}(n)}{\partial W_{o c}(n)}=e_{c}(n) \cdot \nabla g_{c} \cdot s_{c}(n-1)^{\prime}-\gamma_{c} \cdot \frac{\partial y(n+1)}{\partial y(n)} \cdot e_{c}(n) \cdot \nabla g_{c} \cdot s_{c}(n)^{\prime} \\
\frac{\partial E_{c}(n)}{\partial W_{s c}(n)}=\nabla f_{c} \cdot W_{o c}(n)^{\prime} \cdot \nabla g_{c} \cdot e_{c}(n) \cdot s_{c}(n-2)^{\prime}-\gamma_{c} \cdot \nabla f_{c} \\
\cdot W_{o c}(n)^{\prime} \cdot \nabla g_{c} \cdot \frac{\partial \tilde{y}(n+1)}{\partial y(n)} \cdot e_{c}(n) \cdot s_{c}(n-1)^{\prime}(7.19) \\
\frac{\partial E_{c}(k)}{\partial W_{i c}(k)}=\nabla f_{c} \cdot W_{o c}(n)^{\prime} \cdot \nabla g_{c} \cdot e_{c}(n) \cdot s_{c}(n-1)^{\prime}-\gamma_{c} \cdot \nabla f_{c} \\
\cdot W_{o c}(n)^{\prime} \cdot \nabla g_{c} \cdot \frac{\partial y(n+1)}{\partial y(n)} \cdot e_{c}(n) \cdot s_{c}(n)^{\prime}(7.20)
\end{array}
$$

The derivatives of the hidden and output layer functions are $\nabla f$ and $\nabla g$, respectively. The critic network is trained online together with action and model networks simultaneously since the procedure involves the derivatives from both model and action networks. $A(n+1)$ and $y(n+1)$ are obtained from action and model network, respectively, and are used in (13) to obtain $e_{c}(n+1)$.The weights of critic network are updated by using (7.15)-(7.17). The critic network is said to converge when the error defined in (9) is within tolerance, and provides the derivatives of $J[y(n+1)]$ with respect to $y(n+1)$.

\subsubsection{Model Network Design}

The model network predicts the future state of the system from the current state and the current control action as shown Fig.7.5, 


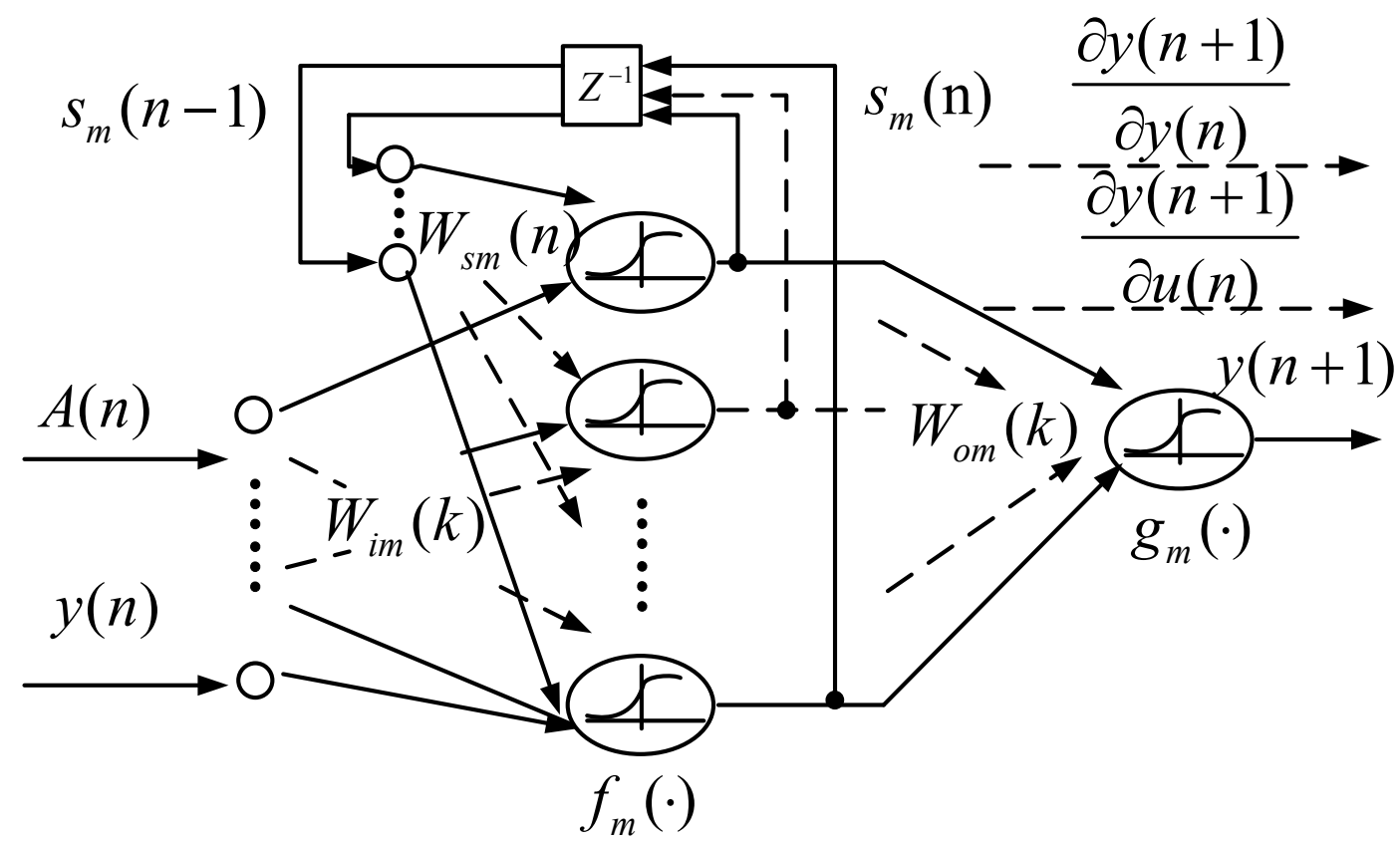

Fig.7.5 The structure of model network

The model network consists of three layers: an input layer with two input vectors $A(n)=$ $\left[\mathrm{P}_{\text {pvset }}, \mathrm{Q}_{\text {pvset }}, \mathrm{P}_{\text {windset }}, \mathrm{Q}_{\text {windset }}\right]$ and $\mathrm{y}(\mathrm{n})$, a hidden layer with 20 neurons, and an output layer with output vector $y(n+1)$ which represents the future system response. The approximation can only be accurate if the error $E_{m}(n)$ is minimized,

$$
E_{m}(n)=e_{m}^{2}(n)=\|\hat{y}(n)-y(n)\|^{2}
$$

where, $\hat{y}(n)$ is the one step delayed the model network output $y(n+1)$. Similar to critic network, the output of model network is a nonlinear weighted sum of system responses and control actions. Backpropagation method is used to update the weights of model network, and the error gradients of model network are given by (7.22) - (7.24),

$$
\begin{gathered}
\frac{\partial E_{m}(n)}{\partial W_{o m}(n)}=\nabla g_{m} \cdot e_{m}(n) \cdot s_{m}(n)^{\prime} \\
\frac{\partial E_{a}(n)}{\partial W_{s m}(n)}=\nabla f_{m} \cdot W_{o m}(n)^{\prime} \cdot \nabla g_{m} \cdot e_{m}(n) \cdot s_{m}(n-1)^{\prime} \\
\frac{\partial E_{a}(n)}{\partial W_{i m}(n)}=\nabla f_{m} \cdot W_{o m}(n)^{\prime} \cdot \nabla g_{m} \cdot e_{m}(n) \cdot y_{m}(n-1)^{\prime}
\end{gathered}
$$

The training of model network doesn't need derivatives from other networks. It can be pretrained offline using the historical data of control signals and system responses utilizing the 
pre-training operation point in Table 7.1. The pre-training of model network accelerates the training convergence speed of system controller. The model network can approximate the system dynamics over a wide operation range. The model network is said to converge when the error in (7.22) - (7.24) is within tolerance, and is used to train the action and critic networks online by utilizing a relatively small learning factor. More details about training data selection can be found in $[88,115]$.

TABLE 7.1

PRE-TRAINING OPERATION POINTS (OP)

\begin{tabular}{llllll}
\hline OP & Insolation $\left(\mathrm{W} / \mathrm{m}^{2}\right)$ & $\begin{array}{l}\text { Ppv_ref } \\
(\mathrm{MW})\end{array}$ & Wind speed $(\mathrm{m} / \mathrm{s})$ & $\begin{array}{l}\text { Pwind_ref } \\
(\mathrm{MW})\end{array}$ & Load \\
1 & 1000 & MPPT & 11.355 & MPPT & constant \\
2 & 1000 & MPPT & 11.355 & MPPT & +0.7 Mvar \\
3 & 900 & MPPT & 10 & MPPT & constant \\
4 & 800 & MPPT & 11.355 & MPPT & constant \\
5 & 1000 & MPPT & 10 & MPPT & constant \\
6 & 5 s actual data & MPPT & 11.355 & MPPT & constant \\
7 & 1000 & MPPT & 5 s actual data & MPPT & constant \\
8 & 5 s actual insolation data & MPPT & 5 s actual wind data & MPPT & constant \\
\hline
\end{tabular}

\subsubsection{Action Network Design}

The action network determines the control action at next time step that can minimize the cost-to-go function obtained from critic network as seen in Fig.7.6.

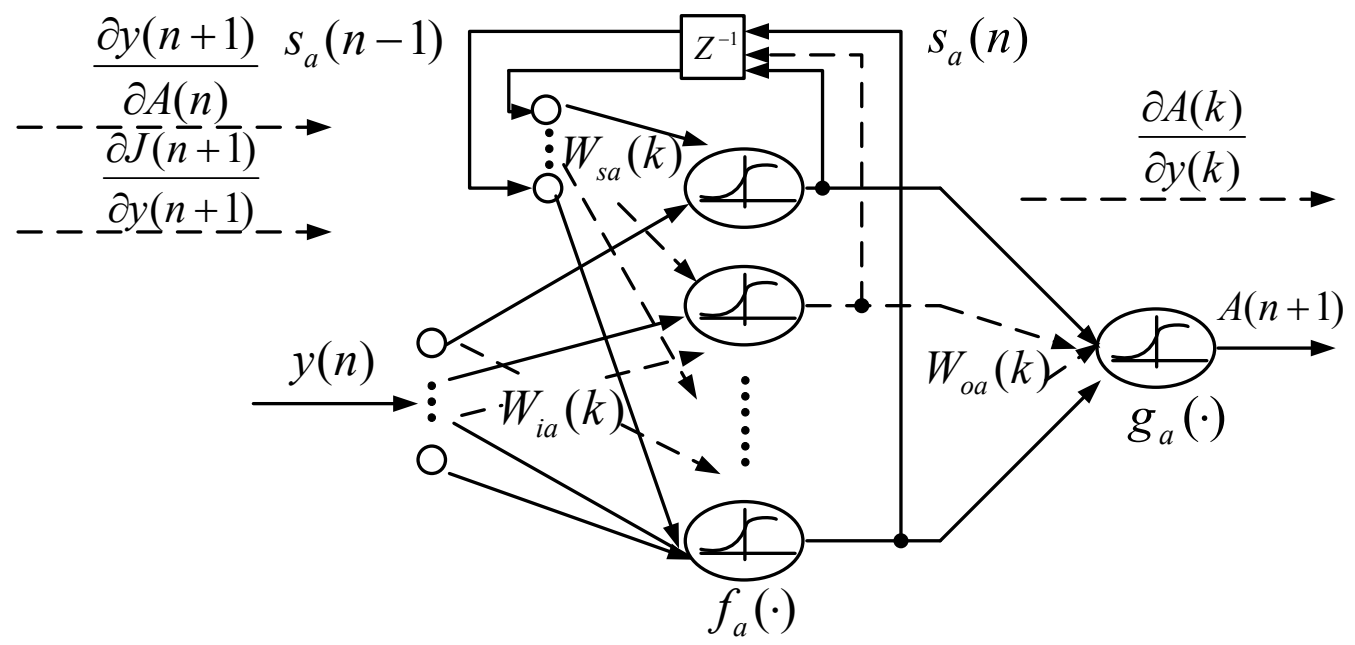

Fig.7.6 The structure of action network

The action network consists of three layers: an input layer with an input vector $y(n)$, a hidden layer with 20 neurons, and an output layer with output vector $\mathrm{A}(\mathrm{n}+1)$. The action network determines a sequence of control actions to minimize $J(n+1)$ at each time step by minimizing the error in (7.25), 


$$
E_{a}(n)=\left|\frac{\partial \hat{J}(n)}{\partial A(n)}\right|^{2}
$$

In (7.25), the derivatives of cost-to-to function with respect to the control action are given by (7.26),

$$
\frac{\partial \hat{J}(n)}{\partial A(n)}=\frac{\partial(U(n))}{\partial A(n)}+\gamma \cdot \tilde{\lambda}(n+1)\left[\frac{\partial(y(n+1))}{\partial A(n)}\right.
$$

The action network converges when the error defined by (25) is within tolerance. Backpropagation method is used here, and the gradients of action network are given by (7.27) - (7.29),,

$$
\begin{gathered}
\frac{\partial E_{a}(n)}{\partial W_{o a}(n)}=\nabla g_{a} \cdot \frac{\partial \hat{\jmath}(n)}{\partial A(n)} \cdot s_{a}(n)^{\prime} \\
\frac{\partial E_{a}(n)}{\partial W_{s a}(n)}=\nabla f_{a} \cdot W_{o a}(n)^{\prime} \cdot \nabla g_{a} \cdot \frac{\partial \hat{J}(n)}{\partial A(n)} \cdot s_{a}(n-1)^{\prime} \\
\frac{\partial E_{a}(n)}{\partial W_{i a}(n)}=\nabla f_{a} \cdot W_{o a}(n)^{\prime} \cdot \nabla g_{a} \cdot \frac{\partial \hat{\jmath}(n)}{\partial A(n)} \cdot y(n)^{\prime}
\end{gathered}
$$

More information on DHP action network training can be found in [88].

\subsection{Local Layer Control Design}

The set points of total injected active and reactive power for the converters is determined by the local layer, here are separate local layer controllers for wind and PV set. The control for battery utilizes the following prescriptions to improve battery life:

1) Discharging is activated if $P_{\text {battset }_{\text {act }}}$ or $Q_{\text {battset }}>\varepsilon$;

2) Charging is activated if $P_{\text {battset }_{\text {act }}}$ or $Q_{\text {battset }_{\text {act }}}<-\varepsilon$;

3) Battery is charged fully unless discharging mode is activated to avoid excessive charging or discharging frequently.

The limits on the inverter capacity require the coordinated control of active and reactive power distribution. Five operation modes of coordinated control are used for wind and PV set as shown in Error! Reference source not found. and explained here. 


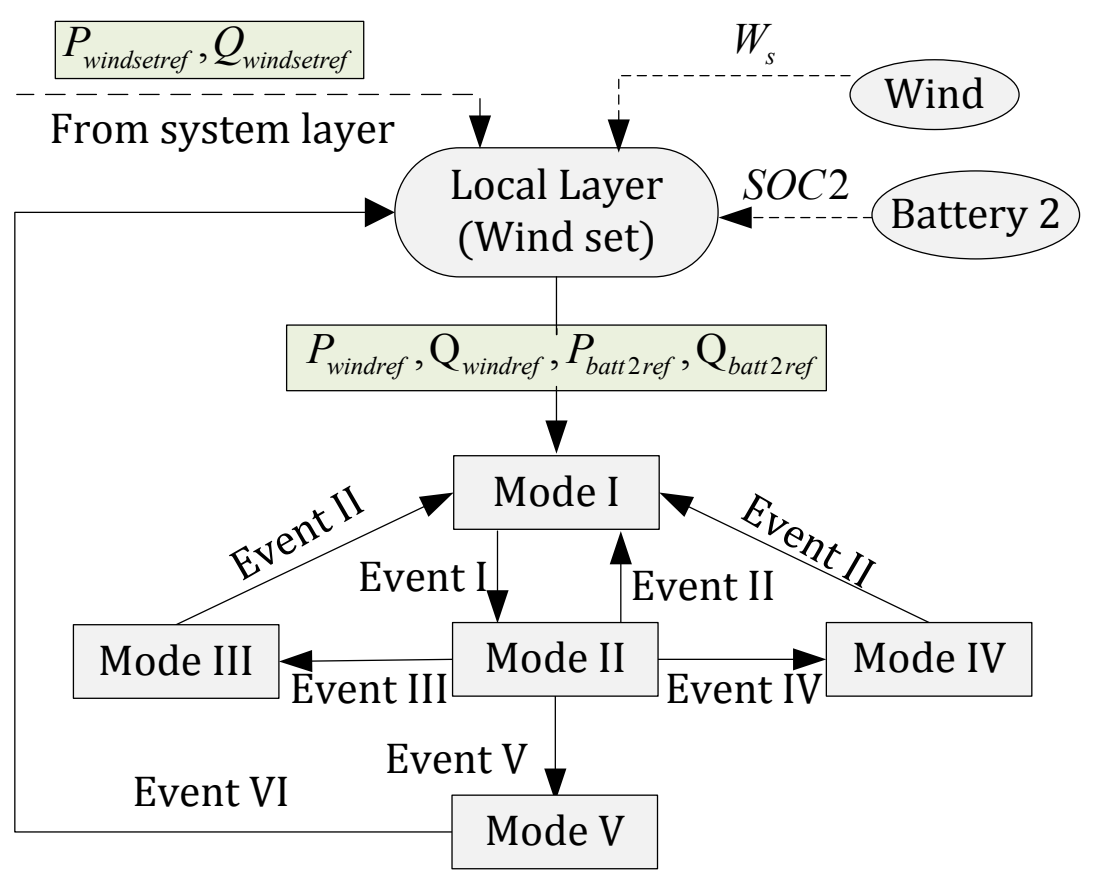

Fig.7.7 Local control layer configuration of the wind set

\section{A. Mode I: Normal Operation Mode}

Wind converter is in MPPT (MPPT can be overridden if reactive power deficiency becomes the primary issue) for maximum power efficiency. The power mismatch between dispatch command and actual output is compensated by battery storage and reactive power is compensated by wind converter. Battery does not compensate for reactive power regardless in this mode. Small transient disturbances are mitigated by the wind converter thereby improving the life cycle of battery storage.

\section{B. Mode II: Wind and Battery Mode}

Mode II is active if the PCC voltage violates the limit and $\left|Q_{\text {windset }_{\text {ref }}}\right|$ is greater than the reactive power capacity of wind converter under MPPT (Event I). The wind converter and the battery inverter compensate the reactive power by droop control to maintain the voltage at PCC bus. When the $\left|Q_{\text {windset }_{r e f}}\right|$ is less than the reactive power capacity of wind converter (Event II), there is no reactive power compensation from battery and the system returns to Mode I.

C. Mode III: Stall Mode 
Mode III is activated exclusively from Mode II, when the PCC voltage violates the high limit and $\left|Q_{\text {windset }_{\text {ref }}}\right|$ is larger than the total reactive power capacity of wind and battery when wind converter is under MPPT (Event III). Wind turbine operation is changed to stall regulation, and the MPPT is overridden. The blade pitch angle is increased to reduce the wind energy capture to reduce the active power output of the wind turbine and increase the maximum reactive power capability of wind converter. This mode remains activated until Event II occurs.

\section{Mode IV: Shedding Mode}

Mode IV is activated exclusively from Mode II, when the PCC voltage violates the low limit and $\left|Q_{\text {windset }_{\text {ref }}}\right|$ is larger than the total available capacity of wind and battery while wind converter is working under MPPT (Event IV). Non-critical loads are disconnected sequentially based on their priorities for voltage recovery. This mode remains activated until Event II occurs.

\section{E. Mode V: Protection Mode}

Mode V is activated when the PCC voltage violates the protection limits (Event V). In order to protect the power electronics devices from over or under voltages and other safety issues, both the wind and battery system are disconnected from the power grid. This mode is active until PCC voltage is in limit (Event VI).

Time and voltage dead-bands of the coordinated controller prevent the excessive mode changes and preserve reactive power. For instance, during Mode I, when the reactive power

dispatch command $\left|Q_{\text {windset }_{\text {ref }}}\right|$ is higher than the maximum reactive power capability of wind converter but the PCC voltage is within voltage dead-band, mode change does not occur and the system remains in mode I to reserve reactive power of battery system. Similarly, PV set- PV and battery work under coordinated control modes.

\subsection{MPC Based Device Layer Control Design}

The firing sequence is controlled by the device layer controls using control signals from local layers. The device layer uses model predictive control that increases the tracking accuracy of the converter control and ensures the controller adaptive. MPC with prediction horizon N=1 
is used here, and the controllers for wind farm converter, PV converter and battery inverters are designed in this section.

\subsubsection{Wind Farm Converter}

A direct drive wind turbine model developed in previous work $[116,109]$ is used as shown in Fig.7.8.

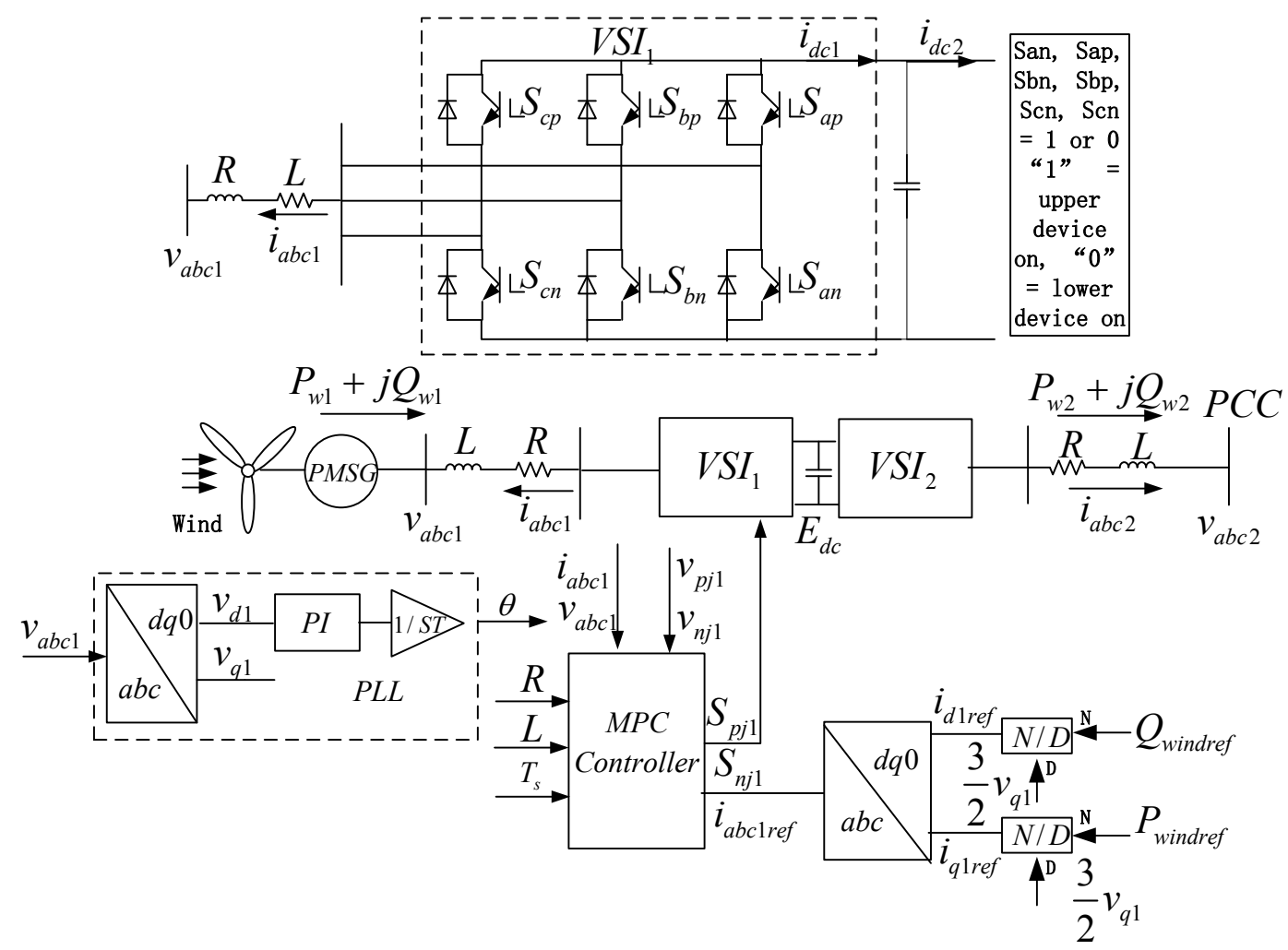

Fig.7.8 Overall control of wind farm

The wind generator has a gearless direct drive wind turbine, a multi-pole Permanent Magnet Synchronous Generator (PMSG), and an AC-DC-AC converter. The wind local layer control captures the maximum wind energy and delivers desired reactive power to the grid utilizing the Voltage Source Inverters (VSI) on both sides of the DC bus.

Assuming the three phase voltage is balanced, and the dynamic behavior of $\mathrm{VSI}_{\mathrm{k}}$ phase $\mathrm{j}$ can be expressed as in equations (7.30) and (7.31), where $V$ and $i$ are the voltage and current of $\mathrm{VSI}_{\mathrm{k}}$ respectively, $\mathrm{V}_{\mathrm{p}}$ and $\mathrm{V}_{\mathrm{n}}$ are the upper and lower bridge voltages, respectively, jk denotes phase $\mathrm{j}$ of $\mathrm{VSI}_{\mathrm{k}}$, and $\mathrm{E}_{\mathrm{dc}}$ is the dc bus voltage.

$$
V_{j k}+L \frac{d i_{j k}}{d t}+R i_{j k}+V_{p j k}=\frac{E_{d c}}{2}
$$




$$
V_{j k}+L \frac{d i_{j k}}{d t}+R i_{j k}-V_{n j k}=\frac{E_{d c}}{2}
$$

From (30) with (31), (32) is obtained

$$
\frac{d i_{j k}}{d t}=\frac{V_{n j k}-V_{p j k}-2 R i_{j k}-2 V_{j k}}{2 L}
$$

Based on the switching functions, $V_{\mathrm{pjk}}$ and $\mathrm{V}_{\mathrm{njk}}$ can be expressed as in (7.33), where, $\mathrm{S}_{\mathrm{p}}$ and $S_{n}$ represent the switching states of upper and lower bridge arm, respectively. In a 2-level VSI used in this paper, there are a total of $\mathrm{C}_{2}^{1}$ available switching states for each phase. The switching states are obtained by MPC based device layer control to carry out the control signals from local layer control. The dc bus voltage $E_{d c}$ can be calculated using (7.34).

$$
\begin{gathered}
\left\{\begin{array}{l}
{\left[\begin{array}{l}
V_{p j k} \\
V_{n j k} \\
S_{n j k}
\end{array}\right]=\left[\begin{array}{c}
0 \\
E_{d c} \\
0
\end{array}\right], S_{p j k}=1} \\
{\left[\begin{array}{l}
V_{p j k} \\
V_{n j k} \\
S_{n j k}
\end{array}\right]=\left[\begin{array}{c}
E_{d c} \\
0 \\
1
\end{array}\right], S_{p j k}=0}
\end{array}\right. \\
i_{d c 1}-i_{d c 2}=C \frac{d E_{d c}}{d t}
\end{gathered}
$$

By regulating the ac current $\mathrm{i}_{\mathrm{jk}}$, the generator side $\mathrm{VSI}_{1}$ captures maximum wind energy as well as maintains zero reactive power exchange. The predicted wind generation is determined by the wind speed $V_{w}$, the mechanical speed of wind turbine $\omega_{\mathrm{w}}$, and the power coefficient $C_{p}$ which is a function of $\omega_{w}, V_{w}$ and blade pitch angle $\beta$. The wind generation and torque with respect to wind speed can be described as (7.35), where $\mathrm{T}_{\mathrm{w}}$ is the mechanical torque of wind turbine, $\rho$ is the air density, $\mathrm{R}$ is the blade radius, and $\beta$. $\mathrm{C}_{\mathrm{p}_{-} \text {opt }}$ is the optimum power coefficient and captures the maximum wind energy $\mathrm{P}_{\text {wind_opt }}$. The approximation of $C_{p_{-} o p t}$ used in this paper can be found in [105]. The power exchange between wind turbine and generator side VSI in next time step can be expressed in synchronous dq0 reference frame as in (7.36).

$$
T_{w}=\frac{P_{w_{-} o p t}}{\omega_{w}}=\frac{1}{2} \rho \pi R^{2} C_{p_{-} o p t} \frac{V_{w}^{3}}{\omega_{w}}
$$




$$
\left[\begin{array}{l}
P\left(t+T_{s}\right) \\
Q\left(t+T_{s}\right)
\end{array}\right]=\left[\begin{array}{c}
\frac{1}{2} \rho \pi R^{2} C_{p} V_{w}^{3}\left(t+T_{s}\right) \\
0
\end{array}\right]=\left[\begin{array}{l}
\frac{3}{2} v_{q}\left(t+T_{s}\right) i_{q}\left(t+T_{s}\right) \\
\frac{3}{2} v_{q}\left(t+T_{s}\right) i_{d}\left(t+T_{s}\right)
\end{array}\right]
$$

where $d$ and $q$ denote the $d$-axis and q-axis sequence, respectively. $T_{s}$ is the time step. By selecting a sufficiently small $\mathrm{T}_{\mathrm{s}}, \mathrm{v}_{\mathrm{q}}\left(\mathrm{t}+\mathrm{T}_{\mathrm{s}}\right)$ and $\mathrm{V}_{\mathrm{w}}\left(\mathrm{t}+\mathrm{T}_{\mathrm{s}}\right)$ can be approximated by the measured value of $v_{q}(t)$ and $V_{w}(t)$. It is seen that the active and reactive power $P\left(t+T_{s}\right)$ and $\mathrm{Q}\left(\mathrm{t}+\mathrm{T}_{\mathrm{s}}\right)$ can be obtained by regulating $\mathrm{i}_{\mathrm{q}}\left(\mathrm{t}+\mathrm{T}_{\mathrm{s}}\right)$ and $\mathrm{i}_{\mathrm{d}}\left(\mathrm{t}+\mathrm{T}_{\mathrm{s}}\right)$, and the reference current in abc-axis at next time step $\mathrm{i}_{\text {ref }}\left(t+\mathrm{T}_{\mathrm{s}}\right)$ can be calculated based on the measurement as shown in Fig.7.8.

The MPC strategy of grid side VSI is so designed to obtain the next time step switching states based on desired active and reactive power output of wind generator. Prediction of next time step current is essential for tracking. Based on (7.32), the dynamic discrete-time model of generator side VSI for current prediction can be deduced with a backward Euler approximation as shown in (7.37),

$$
i_{j k}\left(t+T_{s}\right)=\frac{L}{L+R \times T_{s}} i_{j k}(t)+T_{s} \frac{V_{n j k}\left(t+T_{s}\right)-V_{p j k}\left(t+T_{s}\right)}{2 L+2 R \times T_{s}}-T_{s} \cdot \frac{V_{j k}\left(t+T_{s}\right)}{L+R \times T_{s}}
$$

Since the input of the predict current $i_{j k}\left(t+T_{s}\right)$ belongs to a finite set of switching combinations, a cost function is presented to evaluate the predicted current under all combinations of switching states as shown in equation (7.38),

$$
J_{j k}=\left|i_{j k r e f}\left(t+T_{s}\right)-i_{j k}\left(t+T_{s}\right)\right|
$$

The approach is to reduce the error between predicted and reference current $i_{j k r e f}\left(t+T_{s}\right)$, based upon minimizing $J_{j k}$ with respect to the pool of all switching sequences, and the one that gives the lowest $\mathrm{J}_{\mathrm{jk}}$ is selected to generate the firing pulses for $\mathrm{VSI}_{\mathrm{k}}$. The aforementioned MPC strategy is applied on both VSIs of the wind generator.

\subsubsection{PV Converter}

Real power generated by the PV farm is regulated to deliver maximum active power and desired reactive power to the grid while maintaining the voltage of the coupling capacitor of the PV DC-DC booster and DC-AC inverter as shown in Fig.7.9. The mathematical mode and 
MPC formulation of VSI of direct drive wind turbine can be used for PV system as the PV is connected to the system through similar to wind turbine. A similar type MPC based device layer controller as introduced in session V-A is used for the PV converter to obtain the switching states.

Real power generated by the PV farm is regulated to deliver maximum active power and desired reactive power to the grid while maintaining the voltage of the coupling capacitor between the PV DC-DC booster and the PV DC-AC inverter as shown. Since the PV is connected to the system through voltage source inverter similar to the grid-side inverter of direct drive wind turbine. The mathematical model and the MPC formulation of VSI can be used for PV system. A similar type MPC based device layer controller as introduced in session V-A is implemented in PV converter to generate the firing pulses.

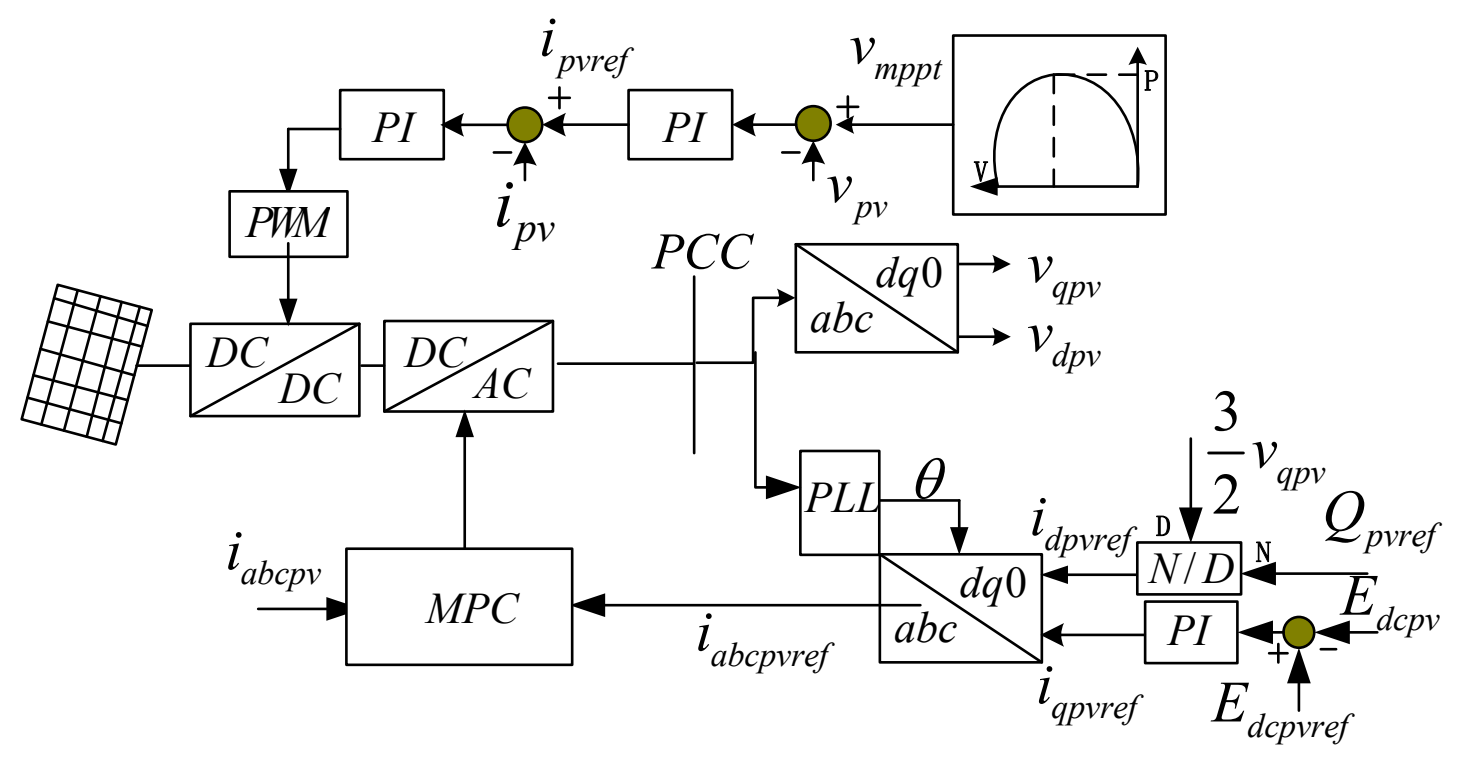

Fig.7.9 Overall control of PV farm

\subsubsection{Battery Inverter}

The control scheme of battery inverter is similar to PV inverter as shown in Fig.7.10. The device controller of battery inverter determines the switching states of battery inverter and communicates the SOC of battery to system control layer for system wide optimization. The proposed MPC based device layer control further reduces the possibility of instability over a wide range of operating conditions. 


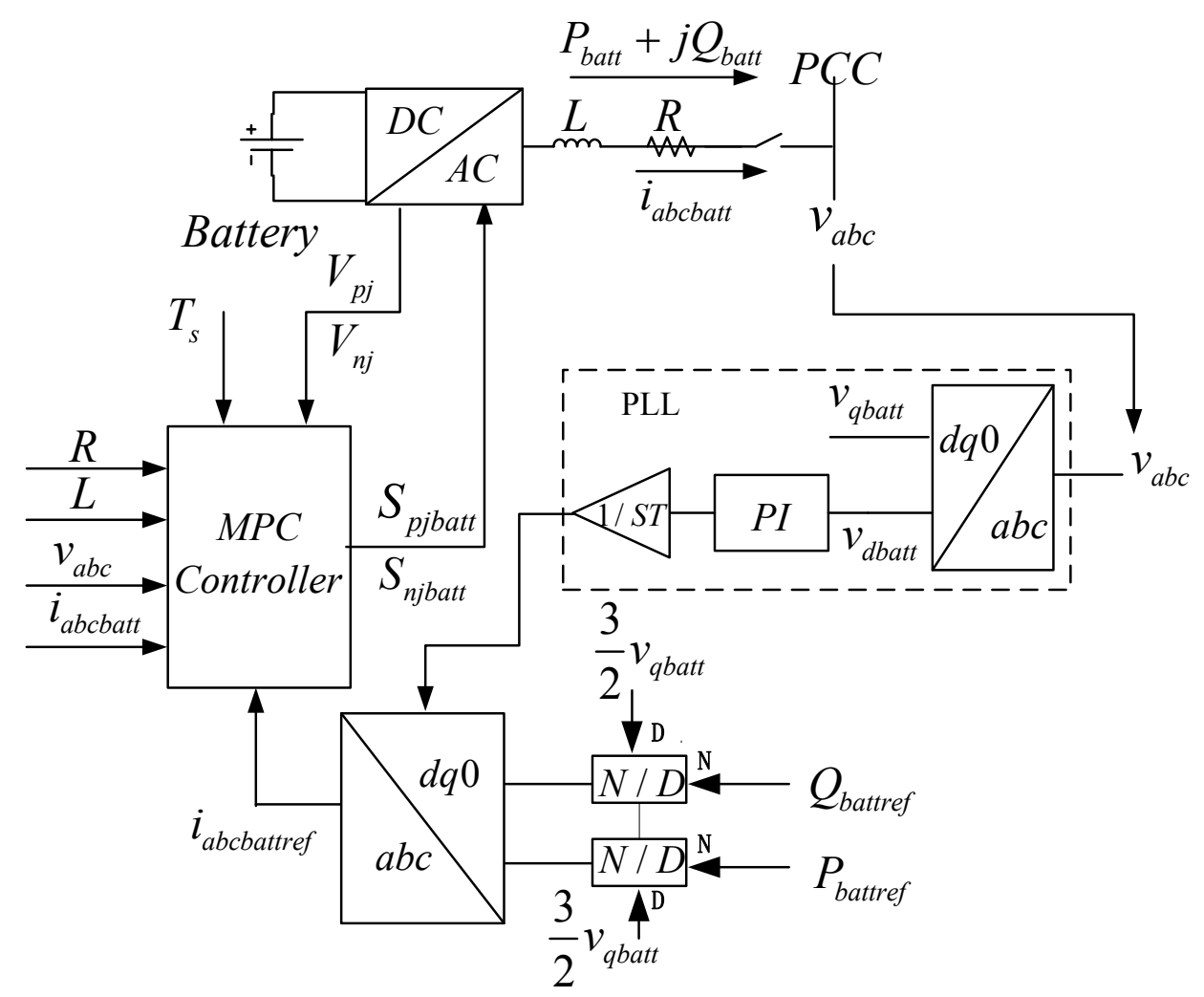

Fig.7.10 Overall control of battery

\subsection{Simulation Results}

Simulation studies are carried out on an IEEE 13 node system in EMTDC/PSCAD to verify the proposed SWAPSC scheme. A direct drive wind generator and a battery storage rated 1.61 MW and 0.5 MW respectively are connected to the system at node 634, and a grid connected PV generator and a battery storage rated $0.8 \mathrm{MW}$ and $0.3 \mathrm{MW}$ respectively are connected at node 680. A set of PV panels connect in shunt and series to obtain a 0.8 MW PV system. Similarly, lead acid batteries of $12.716 \mathrm{~V}$ are connected in shunt and series for MWh level of battery storage. In order to reduce the computational burden, the total simulation duration is limited to $5 \mathrm{~s}$. The SOC variation under this simulation scale is lower and SOC_LL and SOC_UL here are set as $49 \%$ and $51 \%$ to demonstrate the effectiveness of the proposed controller. The SOC_LL and SOC_UL here are not hard limits, and the actual SOC can slightly exceed the limits with initial SOC as 50\%. Here only the results of OP-2 and OP-8 in table 7.1 are presented.

7.5.1. Load Step Change 


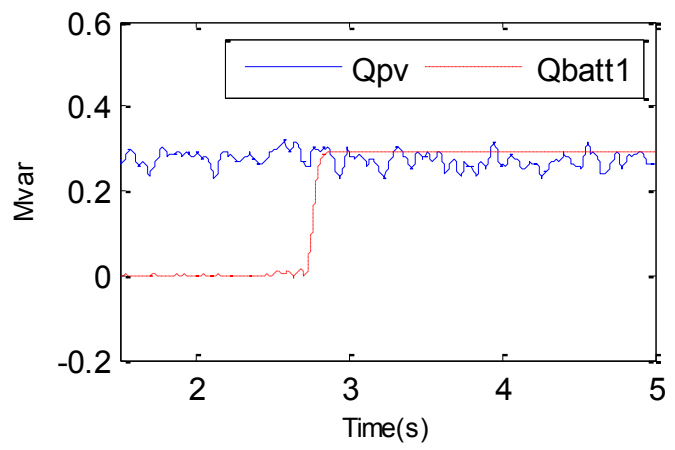

(a)

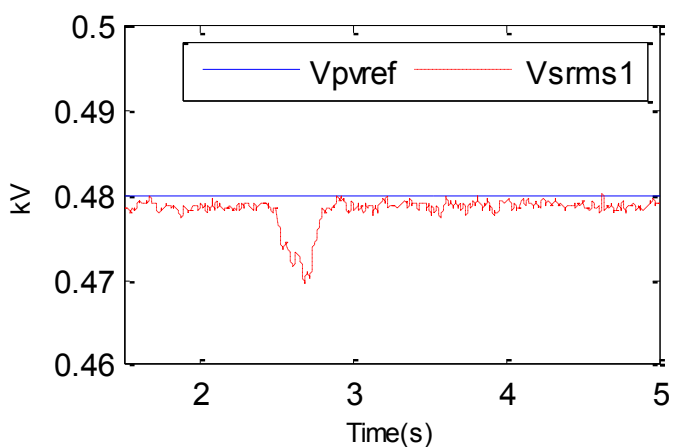

(c)

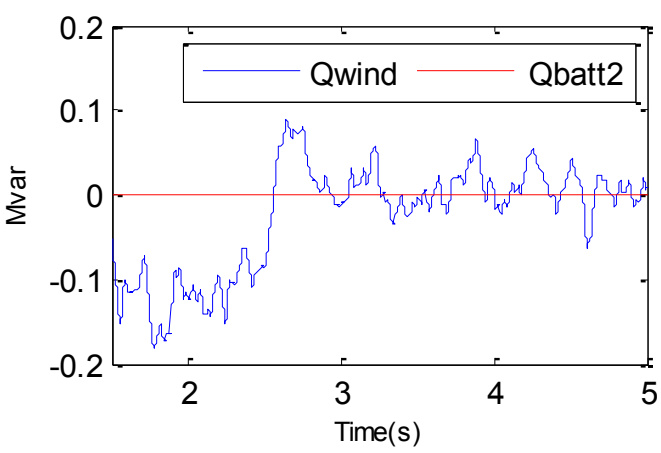

(b)

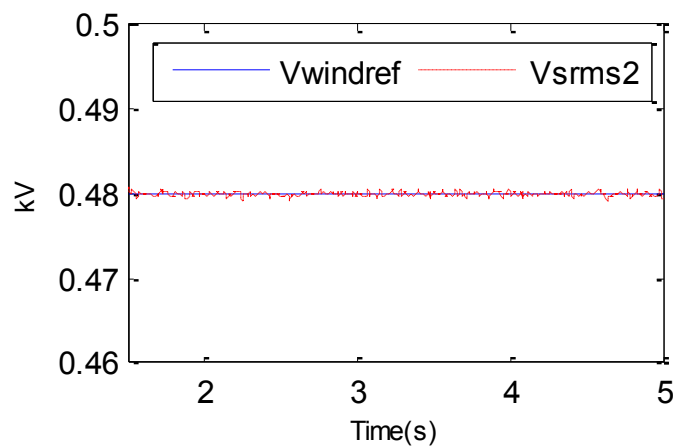

(d)

Fig.7.11 PCC bus voltages with SWAPSC under load step change

The system is running at OP-2 under constant insolation and wind speed as shown in Fig.7.11, with wind reactive power generation of -0.12 Mvar to maintain voltage Vsrms 2 of node 680 at $0.48 \mathrm{kV}$ and $\mathrm{PV}$ at its maximum reactive power output to maintain voltage Vsrms1 at node 680 within limit. As indicated in Section IV, both battery systems are not compensating for reactive power while wind and PV set are working under Mode I (Normal Operation Mode). At 2.5s, a $0.75 \mathrm{MW}+0.42 \mathrm{Mvar}$ load step change is simulated at node 671 . The wind converter increases its reactive power Qwind to $0.02 \mathrm{Mvar}$ to maintain Vsrms2 at $0.48 \mathrm{kV}$ while still in Mode I. However, the reactive power set-point from system control for PV set is higher than maximum reactive power capacity of PV and thus the local layer controls the PV set to mode II (PV and Battery Mode for PV set), increasing battery 1 reactive power output to 0.3 Mvar and restores Vsrms 1 to $0.48 \mathrm{kV}$. 


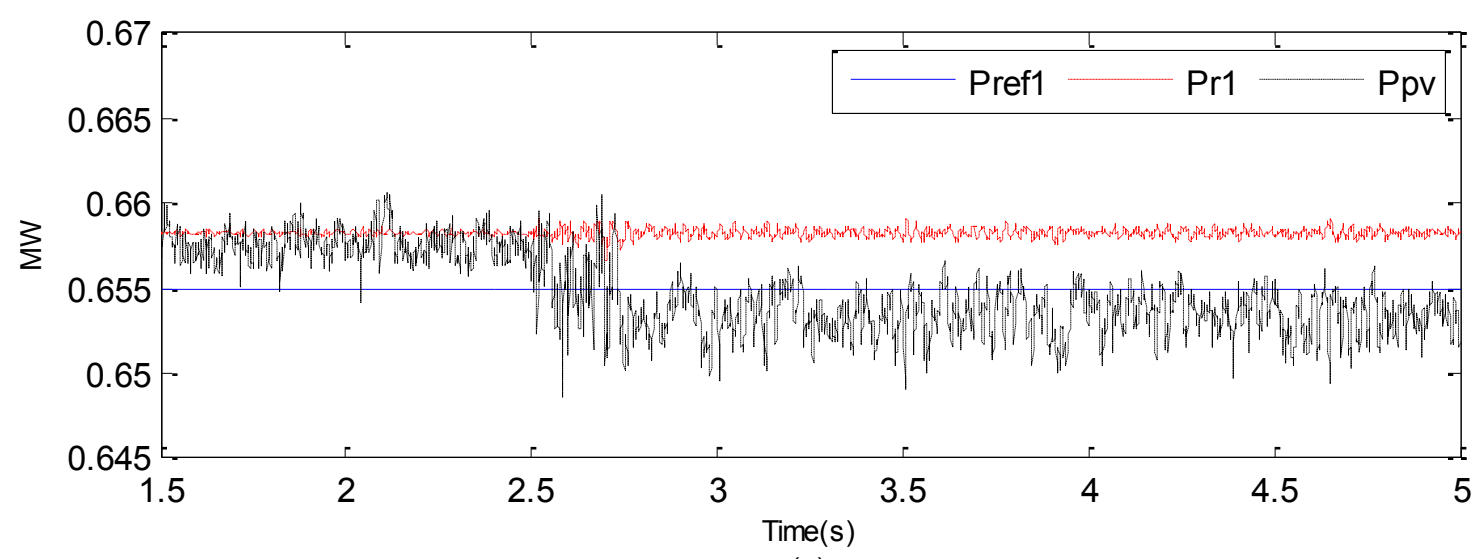

(a)

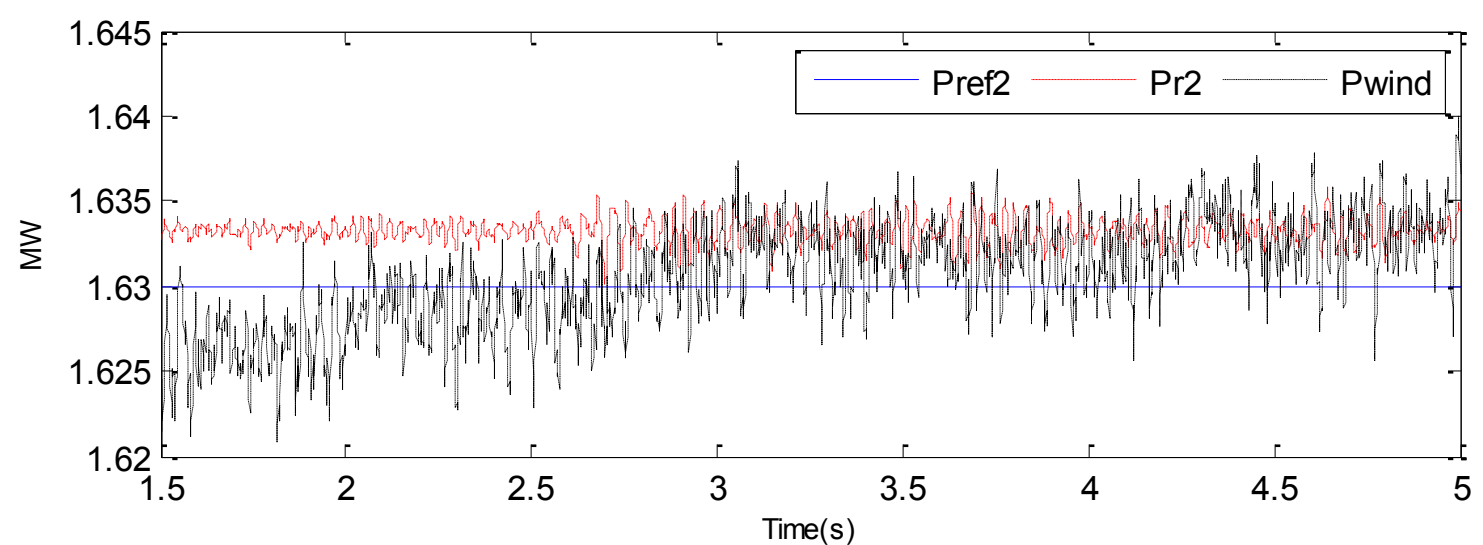

(b)

Fig.7.12 Smoothing of PV and wind farm power with BESS under load step change

Fig.7.12 shows that total injected active power of PV and wind sets Pr1=Ppv+Pbatt1 and Pr2 $=P$ wind $+P$ batt 2 are maintained at 0.658 and $1.633 \mathrm{MW}$ which are slightly higher than the corresponding reference when the load step changes occurs. Less power is generated by grid, resulting in lower system loss Ploss with SWAPSC as seen in Fig.7.13 (f). Since the insolation and wind speed are both constant, the active power outputs of PV and wind Ppv and Pwind are close to the active power dispatch commands Pref1 and Pref2. The active power output of battery 1 and battery 2, Pbatt1 and Pbatt2, are close to 0, and thus SOC1 and SOC2 are within limits as shown Fig.7.13 (a), (b), (d), (e). 


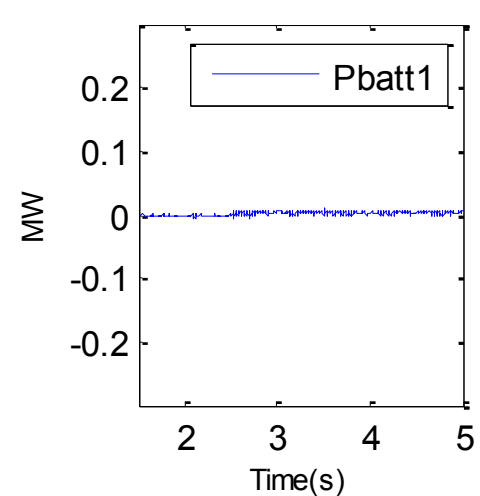

(a)

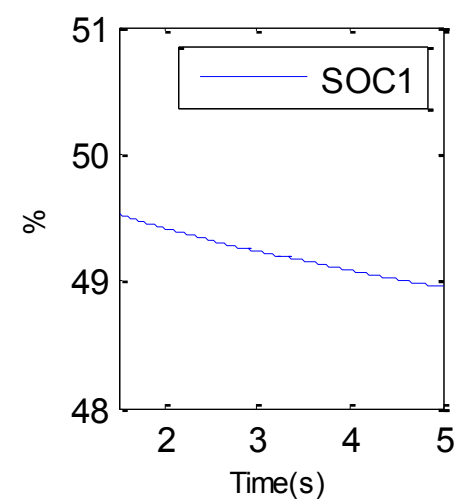

(d)

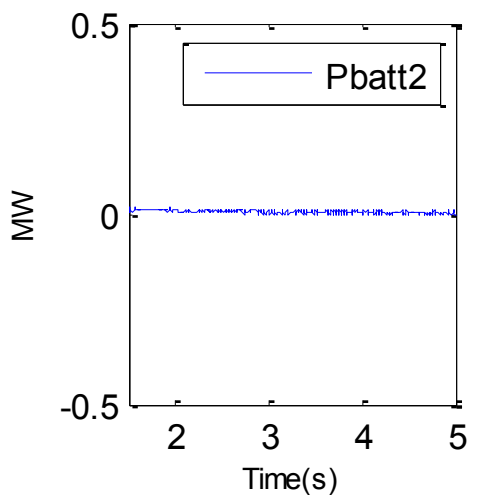

(b)

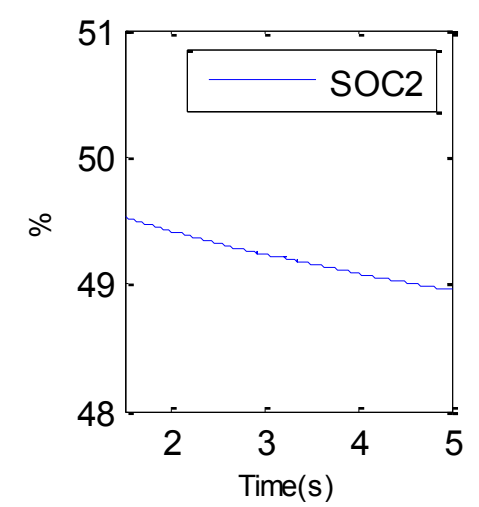

(e)

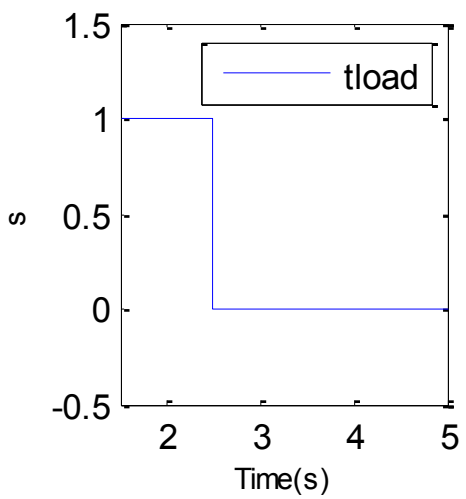

(c)

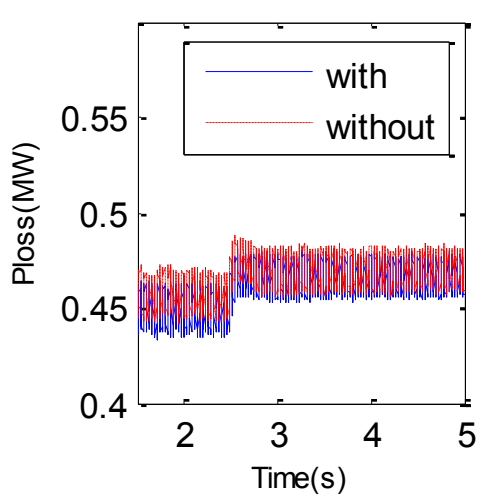

(f)

Fig.7.13 BESS performance with SOC control under load step change

\subsubsection{5 s Actual Insolation and Wind Speed Data}

The system is running under 5 s actual insolation and wind speed data as shown in Fig.7.14 (a) and (b), with wind converter and PV inverter regulating their reactive power outputs to maintain Vsrms1 and Vsrms2 at $0.48 \mathrm{kV}$, and both batteries are not compensating for reactive power as shown in Fig.7.15 (a) - (d). 


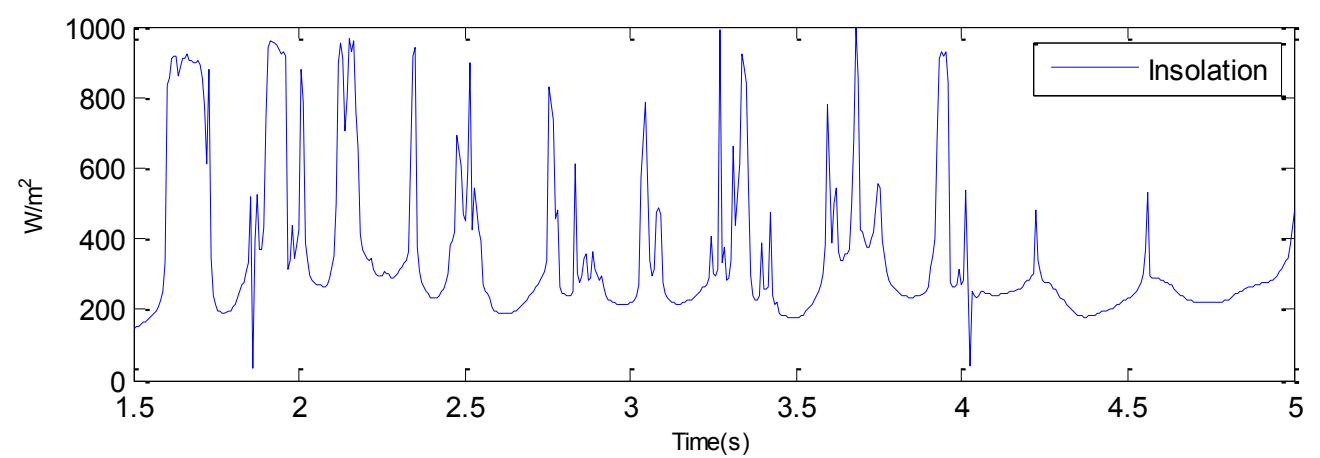

(a)

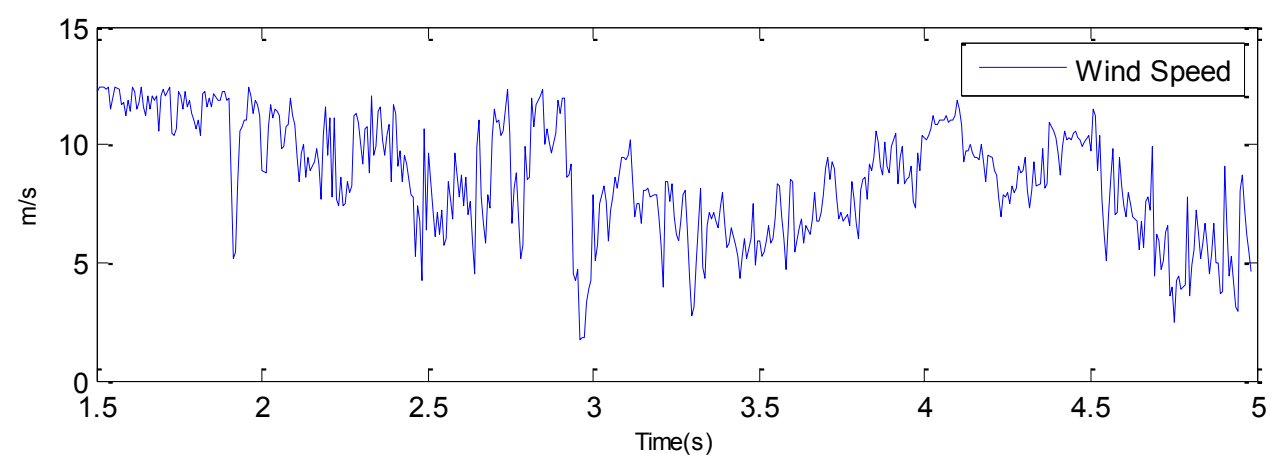

(b)

Fig.7.14 5s insolation and wind speed

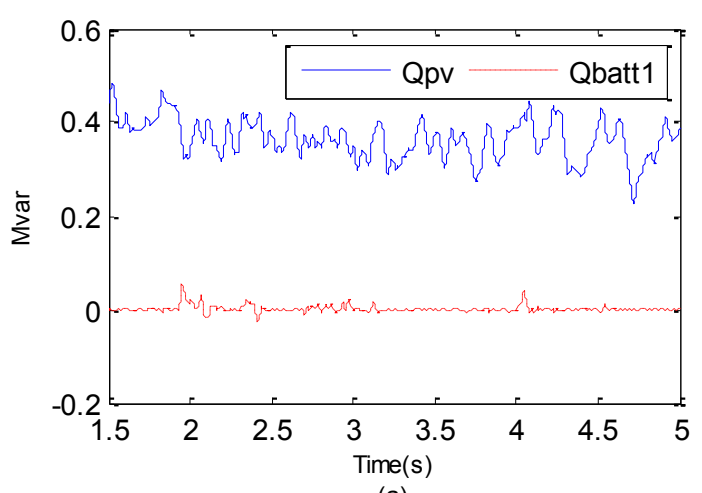

(a)

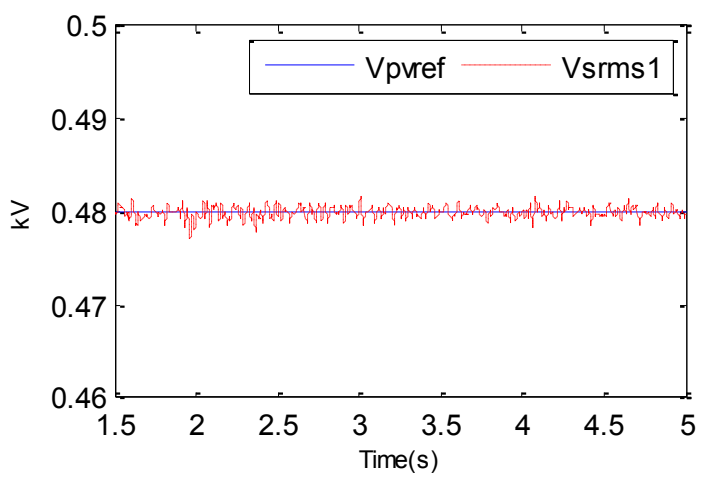

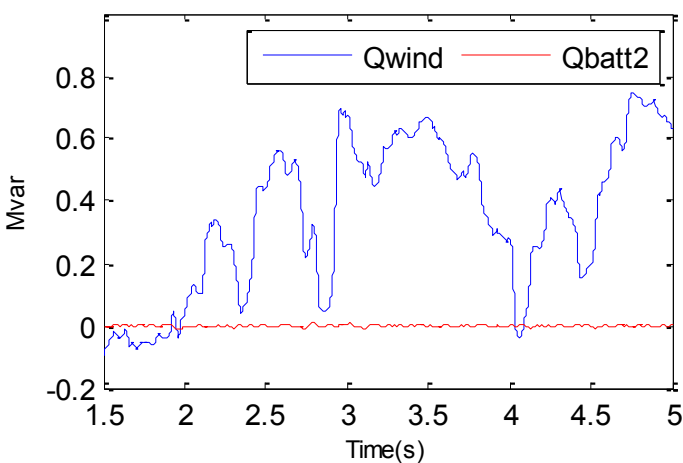

(b)

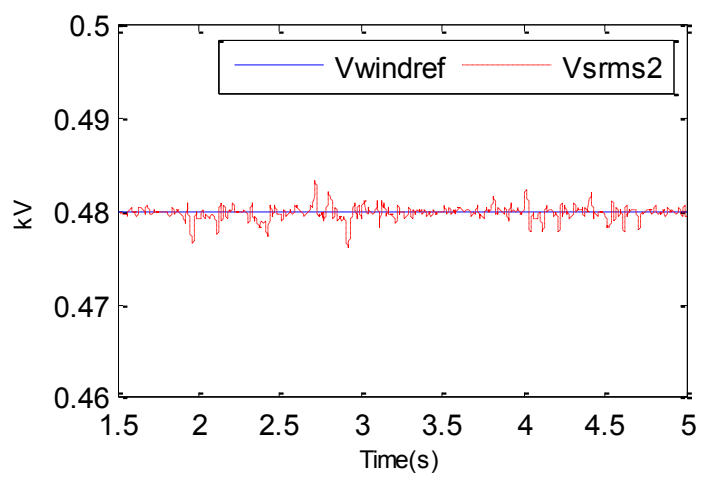

(d)

Fig.7.15 PCC bus voltages with SWAPSC under insolation and wind speed change 


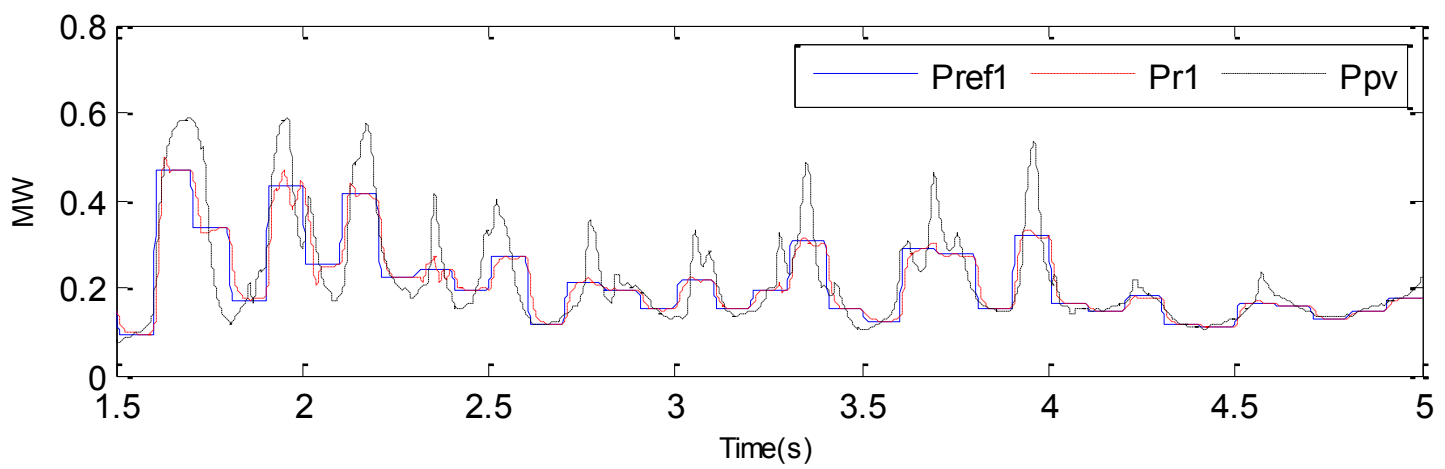

(a)

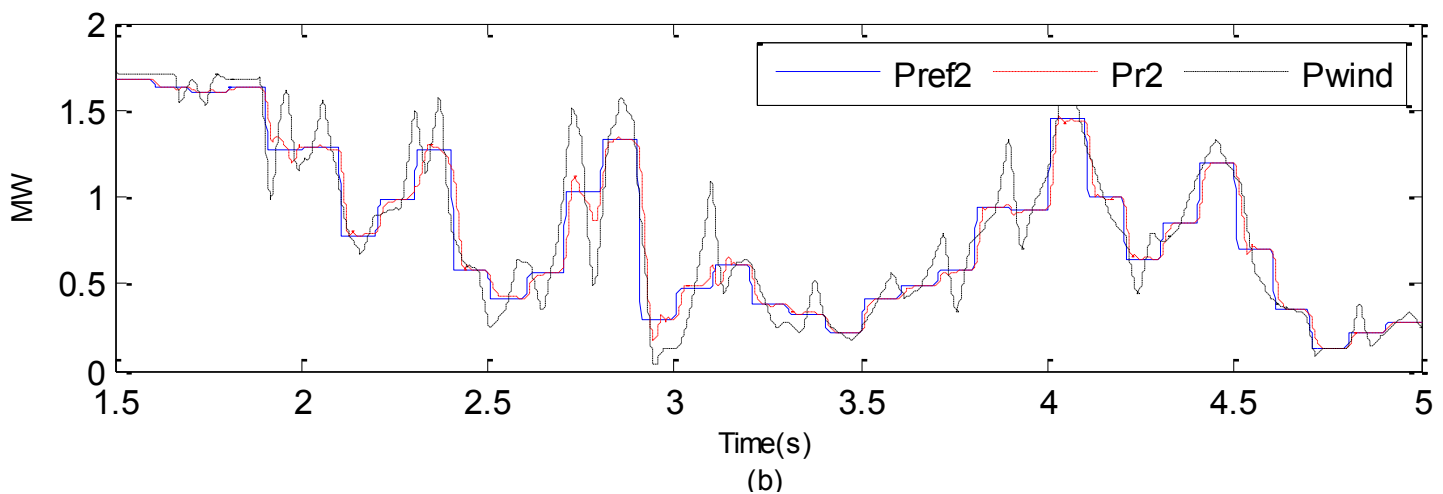

(b)

Fig.7.16 Smoothing of PV and wind farm power with BESS under insolation and wind speed change

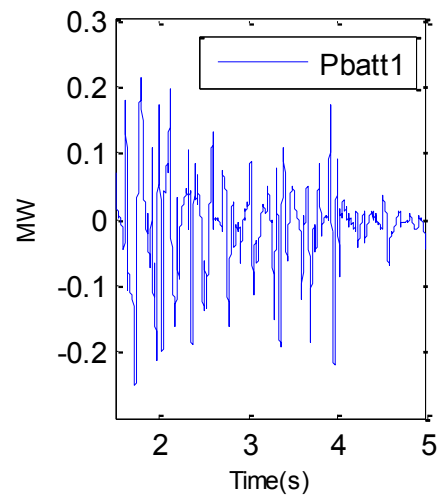

(a)

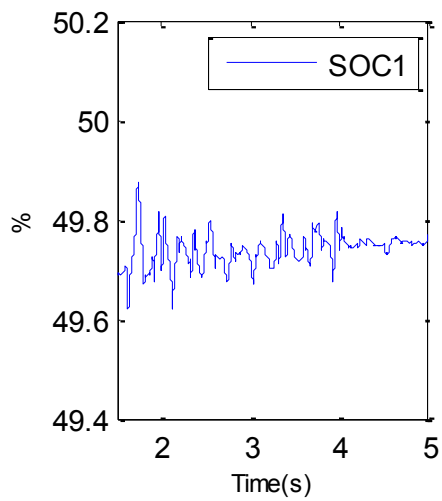

(d)

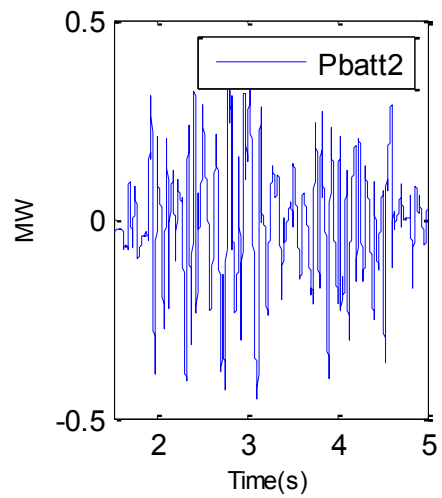

(b)

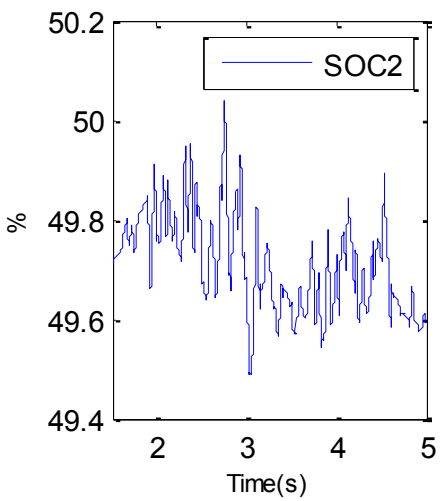

(e)

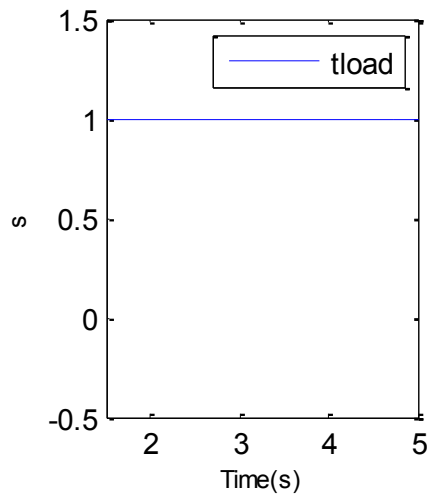

(c)

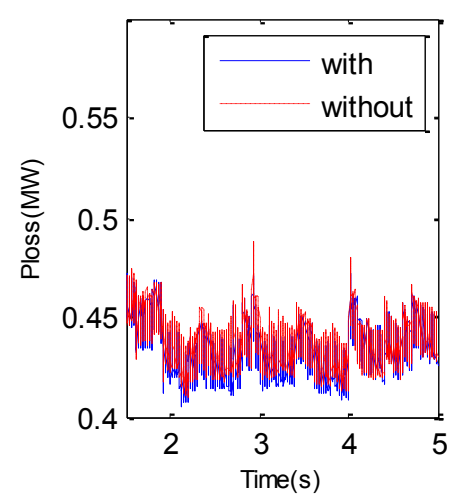

(f)

Fig.7.17 BESS performance with SOC control under insolation and wind speed change 
The active power output of PV Ppv, the reference and actual total injected powers Pref1 and Pr1 using the 5s actual insolation data is shown in Fig.7.16 (a). It is seen that the total injected power of PV set is slightly higher than but follows the trajectory of the desired set points in general; although small deviations occur from time to time when sudden drop or rise in insolation happens or SOC1 exceeds the limit.

The active power output of wind Pwind, the reference and actual total injected powers Pref2 and Pr2 using the 5s actual wind speed data is also shown in Fig.7.17 (a) and (b). It is seen that the total injected power of wind set is slightly higher but follows the trajectory of the desired set points in general; although small deviations occur from time to time when sudden drop or rise in wind speed happens or SOC2 exceeds the limit. Active power outputs of battery 1 and 2 smooth the PV and wind output as shown in Fig.7.17 (a) and (b). , and the output power of battery systems 1 and 2 is limited to rated \pm 0.3 and $\pm 0.5 \mathrm{MW}$, respectively, with SOC1 and SOC2 within limits. It is worth noting that in this study a lower $\mathrm{k}_{\text {loss }}$ for the loss optimization is chosen since a large value of $\mathrm{k}_{\text {loss }}$ can lead to larger mismatch between Pr and Pref. Fig.7.16(f) shows that the system loss is slightly reduced when the proposed SWAPSC is utilized.

It is interesting to compare the results in this work using SWAPSC controller with existing controllers. The existing controllers for active power smoothing or voltage regulation aim to optimize a single objective where as the proposed control scheme considers multi-objectives (active power smoothing, voltage regulation, SOC control, loss reduction and control effort minimization) and hence though the results for a single objective differ only slightly, it is the optimization of multiple objectives that make this controller superior.

\subsection{Chapter Conclusion}

An advanced optimization and intelligent control algorithm for a microgrid with multiple renewable resources has been proposed in this paper that aims at maintaining the smart grid at optimum operating point under various operation conditions such as load variation, insolation change, wind speed change, etc. The proposed SWAPSC scheme consists of a threelayer control scheme: DHP based system layer control, coordination based local layer control, and model predictive control based device layer control. By adjusting the real and reactive powers of the PV, wind and battery systems, the SWAPSC ensures the total injected power from the PV and wind sets are dispatchable. There is improvement in the voltage stability 
range, maximum reactive power is preserved indicated the life span of the batteries, reduction in total system loss with minimum control effort. The detailed design of the DHP and MPC control strategies are presented. Simulation results demonstrate that the proposed control scheme ensures superior performance compared to the existing controllers that tend to optimize a single objective. 


\section{Chapter 8. Summary Conclusion and Contributions}

In summary, this dissertation presents the study on modeling and advanced control strategy design to enhance the performance and reliability of a microgrid with PV and wind integration. The research performed in this work includes the following: 1) the modeling and circuit design of a grid-connected PV system, a direct drive wind turbine, and a lithium-ion type battery system (Chapter 5 \& Chapter 6); 2) a multi-objective controller with Power Quality Control (PQC) and Fault Ride Through Control (FRTC) (Chapter 4); 3) a coordination controller for PV and battery to provide a comprehensive solution to both active and reactive power issues caused by the intermittency of insolation and load change (Chapter 5); 4) an MPC based coordinated predictive controller to provide sustainable power as well as dynamic reactive power support to the load in both grid-connected and islanded operation, thus reducing active power oscillation as well as tracking maximum wind energy (Chapter 6); 5) an ACD based system wide coordinated controller to smoothen the PV and wind generation output, provide dynamic reactive power support, and reduce the power loss as well as maximize the usage of battery storage. Many original contributions have been made on inverter modeling and control as listed,

1) A comprehensive literature review on the existing work related to this study has been conducted in Chapter 2. The topics include power quality control, microgrid modeling, coordinated control design, optimization using GA, and intelligence control techniques. The advantages and disadvantages of the existing work are compared and evaluated.

2) PV inverters normally operate under the rated capacity and hence the remaining capacity of PV inverters can be utilized for power quality and system stability improvement. A multi-objective controller for PV inverter is proposed to compensate for voltage flicker, harmonics, transient voltage, voltage unbalance as well as provide dynamic reactive power support during transient state. The control scheme reduced unnecessary economic investment by enabling the PV inverter to perform STATCOM function. The power quality and the low voltage ride through capability of the PV farm are improved. 
3) Battery storage is necessary in reducing the power variation caused by the intermittency of insolation, and enhancing the coordination between PV and battery provides more reliable power to the grid. In order to further maximize the usage of the battery, a coordinated control scheme is proposed to supervise the output real and reactive power of a battery storage system which adds an extra degree of flexibility to a Microgrid by allowing the temporal separation between generation and consumption of power. The proposed coordinated control can prevent the system from voltage collapse by providing three levels of protection: PV voltage control mode, PV and battery voltage control mode, and load shedding and RMPPT mode. The three levels of protection optimize PV and battery inverter performance without influencing the life span of battery storage. A novel real-time experimental method for connecting the physical PV panel and the battery storage is also proposed to establish a Hardware in the Loop microgrid experimental platform consisting of a physical PV system and battery storage for control strategy test.

4) An MPC based comprehensive coordinated control scheme is proposed for a microgrid with wind and battery to provide sustainable power as well as dynamic reactive power support to the load. A transition method is also implemented to avoid the phase shift in PCC bus voltage during islanding or re-connecting. With the proposed control strategy, the wind farm is capable of operating at maximum power point in both grid-connected and islanded mode with minimum active power variations. Dynamic simulations show that the MPC strategy increases the accuracy of the wind converter and reduces the response time of the wind system.

5) The dynamic stochastic optimal control design for a microgrid with PV and wind integration is proposed to handle the fast insolation and wind speed variation by coordinating all renewable resources and battery storages in the power grid. The aims of the control scheme are to make the active power output of PV and wind dispatchable and hence reduce the size of battery storage, increase the voltage stability in the local region, make the battery systems operate within the safety range, and reduce the total line losses of the system. A three layer hierarchy structure is applied to obtain these objectives. The design procedures and all the control rules are presented in detail. Real insolation and wind speed data is used and an IEEE 13 bus feeder is modeled as the test system to demonstrate the system wide optimize 
controller. Simulation results show the promising performance of the proposed controller under various operating conditions. 


\section{Chapter 9. Recommendations for Future Work}

The following suggestions are made for any continuing research based on the results presented in this study.

\subsection{Genetic Algorithm Approaches in PI Parameters Optimization}

The design of a genetic algorithm approach of PI parameter optimization for a system with a single PV inverter is presented. It could be a worthwhile topic if the work can be expanded to a system with multi PVs, and multi wind farms. An average model is required to reduce the computation effort, and a proper weight factor selection is also needed to ensure the convergence of the GA program. One can model both the power system and GA program in MATLAB. But it would be more interesting if one can model the power system in PSCAD, and GA program in MATLAB. Then the establishment of the interface between PSCAD and MATLAB is necessary.

\subsection{Distributed PV Panel Modeling and Control}

The PV model studied in this work is an integrated model which uses a single PV panel to represent a large number of small PV panels connected in shunt and series. The influence of the insolation change on the PV output is treated as a unified one. However, in the real world, the terminal voltage and output power of each PV panel is different which increases the difficulty of MPPT strategy design and may induce circulation current among shunt connected PV panels. One can study the shadow effect on the power quality of the PV farm and how to reduce the circulation current by using MPC.

\subsection{SWAPSC in Regional Voltage Stability Improvement and Load Frequency Control}

Only the voltage stabilities of the two PCC buses where PV and Wind systems connect are considered in the SWAPSC design. Including the voltage stability of all the buses in between can enhance the system wide benefit even more. Moreover, the test system of the IEEE 13 node feeder in this work uses an infinite source in series with an internal impedance to represent the main power grid. The influence of the insolation, wind speed and load change 
on load frequency is minimized due to this setup. A synchronous generator can be used to replace the infinite source and carry out the load frequency control study. The main challenge is that the synchronous generator in PSCAD is a model with high order differential equations and it can be very time consuming for the machine to reach a steady state. Proper designed turbine and governor models could be used to address this issue.

\subsection{SWAPSC in Island Operation}

The proposed SWAPSC scheme only considered the grid-connected operation of the microgrid. By adding the islanded operation, the control idea can be more intriguing. However, the control objectives of the microgrid under grid connected and islanded operation are different. A proper selected utility function is crucial in the design and training of the ACD based system layer control. Since more operation scenarios are involved, one can use an average model and remove the MPC based device layer to reduce the computation effort. 


\section{Chapter 10. Publication}

- "Study of unified control of STATCOM to resolve the power quality issues of a gridconnected three phase PV system.", 2012 IEEE PES Innovative Smart Grid Technologies Conference.

- "Multi-objective Control Scheme to Improve the Performance of Three-phase Gridconnected PV Generation”, 2012 IEEE PES General Meeting.

- "Modeling and Coordinate Controller Design of A Microgrid System in RTDS", 2013 PES General Meeting.

- $\quad$ "Coordinated Predictive Control of a Wind/Battery Microgrid System," IEEE Journal of Emerging and Selected Topics in Power Electronics.

- $\quad$ "A Coordinated Controller Design of a Microgrid in a Hardware in the Loop (HIL) Experimental Platform Using RTDS" under review by IEEE Transactions on Sustainable Energy.

- "Adaptive Critic Design Based Dynamic Stochastic Optimal Control Design for a Microgrid with Multiple Renewable Resources", under review by IEEE Transactions on Smart Grid. 


\section{Chapter 11. Reference}

[1] European Commission Climate Action. The EU climate and energy package. [Online]. http://ec.europa.eu/clima/policies/brief/eu/package_en.htm

[2] U.S. Depatment of Energy report. (2008, July) $20 \%$ wind energy by 2030: increasing wind energy's contribution to U.S. electricity supply. [Online].

http://www1.eere.energy.gov/windandhydro/pdfs/41869.pdf

[3] Y. Zhang, "Study on Operation Characteristics of Grid-connected Doubly Fed Induction Generators," Master Dissertation, Dept. Elec. Eng., North China Electric Power Univ., 2008.

[4] R. C. Dugan, et al., Electrical power systems quality, 3rd ed.: McGraw-Hill, 2012.

[5] Micah Erichson, Robert Lasseter, Integration of Battery-Based Energy Storage Element in the CeRTS Microgrid. Madison, WI: University of Wisconsin-Madison, Oct 27, 2009.

[6] W. Jewell, et al., "Electrical power system quality," IEEE Power and Energy Magazine, vol. 99, pp. 63-64, 2003.

[7] T. Larsson and C. Poumarede, "STATCOM, an efficient means for flicker mitigation," IEEE meeting in Power Engineering Society, vol. 2, pp. 1208-1213, 1999.

[8] H. Chong, et al., "Evaluation of Cascade-Multilevel-Converter-Based STATCOM for Arc Furnace Flicker Mitigation," IEEE Transactions on Industry Applications, vol. 43, pp. 378385, 2007.

[9] M. Joorabian et al., "Voltage flicker compensation using STATCOM," IEEE conference on Industrial Electronics and Applications, pp. 2273-2278, 2009.

[10] A.Albanna and C.J. Hatziadoniu, "Harmonic modeling of three-phase neutral-point inverters," North American Power Symposium, pp. 1-6, 2009.

[11] I. T. Papaioannou et al., "Harmonic impact of small photovoltaic systems connected to the LV distribution network," 5th international Conference on Electricity Market, pp. 1-6, 2008. 
[12] M. C. Benhabib et al., "Harmonic effects caused by large scale PV installations in LV network," 9th International Conference on Electrical Power Quality and Utilisation, pp. 16, 2007.

[13] E. Vasanasong and E.D. Spooner, "The effect of net harmonic currents produced by numbers of the Sydney Olympic Village's PV systems on the power quality of local electrical netork," International Conference on Power system Technology, pp. 1001-1006, 2000.

[14] T. Ito, et al., "Harmonic current reduction control for grid-connected PV generation systems," Internationa Power Electronics Conference, pp. 1695-1700, 2010.

[15] M. Keqilao, et al., "Analysis of sine-wave inverter's harmonic contents by simulation," 2nd International Conference on Mechanical and Electronics Engineering, pp. 155-158, 2010.

[16] J. Schlabbach, "Harmonic current emission of photovoltaic installations under system conditions," 5th International Conference on Electricity Market, pp. 1-5, 2008.

[17] K.K.J. Thongprona, "Effects of low radiation on the power quality of distributed PV-grid connected system," the Solar Cells and Solar Energy Material Symposium, vol. 90, pp. 2501-2508, 2006.

[18] M. Ciobotaru, et al., "On-line grid impedance estimation based on harmonic injection for grid-connected PV inverter," IEEE international Symposium on Industrial Electronics, pp. 2437-2442, 2007.

[19] A. Cetin and M.Ermis, "VSC-Based D-STATCOM With Selective Harmonic Elimination," IEEE Transactions on Industry Applications, vol. 45, pp. 1000-1015, 2009.

[20] A. R. Oliva, et al., "Power-quality monitoring of a PV generator," IEEE Transactions on Energy Conversion, vol. 13, pp. 188-193, 1998.

[21] L. Jing, et al., "Study on unified control of grid-connected generation and harmonic compensation in dual-stage high-capacity PV system," IEEE Conference on Energy Conversion Congress and Exposition, pp. 3336-3342, 2009.

[22] G.J. Vachtsevanos, K.C. Kalaitzakis, "On the Control and Stability of Grid Connected Photovoltaic Sources," IEEE Transactions on Energy Conversion, , vol. EC-2, no. 4, pp. 556562, December 1987.

[23] C.L. Masters, "Voltage rise: the big issue when connecting embedded generation to long 11 kV overhead lines," Power Engineering Journal, vol. 16, pp. 5-12, Feb 2002. 
[24] L.A.C. Lopes, T.H.M. El-Fouly, R. Tonkoski, "Coordinated Active Power Curtailment of Grid Connected PV Inverters for Overvoltage Prevention," IEEE Transactions on Sustainable Energy, vol. 2, no. 2, pp. 139-147, April 2011.

[25] Di Lu H. Kanchev and V. Lazarov, B. Francois, F. Colas, "Energy Management and Operational Planning of a Microgrid With a PV-Based Active Generator for Smart Grid Applications," IEEE Transactions on Industrial Electronics, vol. 58, no. 10, pp. 4583-4592, October 2011.

[26] A. Arulampalam, et al., "Power quality and stability improvement of a wind farm using STATCOM supported with hybrid battery energy storage," IEE Proceedings of Transmission and Distribution Generation, vol. 153, pp. 701-710, 2006.

[27] C. Hochgraf and R.H. Lasseter, "STATCOM controls for operation with unbalanced voltages," IEEE Trans. Power Del., vol. 13, no. 2, pp. 538-544, April 1998.

[28] E. H. Watanabe, and M. Aredes C. A. C. Cavaliere, "Multi-pulse STATCOM operation under unbalanced voltages," IEEE Power Eng. Soc. Conf. in Proc., pp. 567-572, 2002.

[29] Jinjun Liu, Zhaoan Wang, Biao Wei, Kuang Li, "Strategies and Operating Point Optimization of STATCOM Control for Voltage Unbalance Mitigation in Three-phase Three-Wire System," IEEE Transactions on Power Delivery, vol. 22, no. 1, pp. 413-422, January 2007.

[30] G. Joos, and L. T. Moran S. Chen, "Dynamic performance of PWM STATCOMs operating under unbalance and fault conditions in distribution systems," IEEE Power Eng. Soc. Conf. in Proc., pp. 950-955, 2001.

[31] R. Pawelek, I. Wasiak, R. Mienski, "Shunt compensation for power quality improvement using a STATCOM controller: modeling and simulation," IEE Proceedings of Transmission and Distribution Generation, vol. 151, no. 2, pp. 274-280, March 2004.

[32] F. F. Edwin, G. Spagnuolo, J. Jatskevich, Weidong Xiao, "Efficient Approaches for Modeling and Simulating Photovoltaic Power Systems," IEEE Journal of Photovoltaics, vol. 3, no. 1, pp. 500-508, January 2013.

[33] D. Maksimovic, R.W. Erickson, Fundamentals of Power Electronics, 2nd ed.: Springer, 2000.

[34] G. Gupta, D. Kastwar, A. Hussain, H. Ranjan R. Gupta, "Modeling and design of MPPT controller for a PV module using PSCAD/EMTDC," IEEE PES Innovative Smart Grid Technologies Conference Europe, pp. 1-6, October 2010. 
[35] Jiancheng Zhang, Hongbo Wu, Jing Hu, "A novel MPPT control algorithm based on numerical calculation for PV generation systems," IEEE 6th international Power Electronics and Motion Control Conference, pp. 2103-2107, May 2009.

[36] K. Kalaitzakis, N.C. Voulgaris, E. Koutroulis, "Development of a microcontrolller-based, photovoltaic maximum power point tracking control system," IEEE Transactions on Power Electronics, vol. 16, no. 1, pp. 46-54, January 2001.

[37] Daohong Wang, "A method for instantaneous measurement of PV V-I characteristics and its application for MPPT control," 35th IEEE Photovoltaic Specialists Conference, pp. 2025, June 2010.

[38] Mingwei Shan, Liying Liu, J. M. Guerrero Qiang Mei, "A Novel Improved Variable Step-Size Incremental-Resistance MPPT for PV System," IEEE Transactions on Industrial Electronics, vol. 58, no. 6, pp. 2427-2434, June 2011.

[39] Paul C.Krause, et al., Analysis of Electric Machinery.: IEEE Press, 1994.

[40] M. Mazzola, J. Gafford, N. Younan, Jianwei Li, "A new parameter estimation algorithm for an electrical analogue battery model," Twenty-Seventh Annual IEEE Applied Power Electronics Conference and Exposition, pp. 427-433, February 2012.

[41] MathWorks Inc., "MATLAB user's Manual," 1984-2012.

[42] Shengyi Liu, R. A. Dougal, Lijun Gao, "Dynamic lithium-ion battery model for system simulation," IEEE Transactions on Energy Conversion, vol. 21, no. 2, pp. 504-511, June 2006.

[43] Min Chen, G. A. Rincon-Mora, "Accurate electrical battery model capable of predicting runtime and I-V performance," IEEE Transactions on Energy Conversion, vol. 21, no. 2, pp. 504-511, June 2006.

[44] Kai Sun, Li Zhang, Yan Xing, J. M. Guerrero, "A Distributed Control Strategy Based on DC Bus Signaling for Modular Photovoltaic Generation System With Battery Energy Storage," IEEE Transactions on Power Electronics, vol. 26, no. 10, pp. 3032-3045, October 2011.

[45] K. J. Kyriakopoulos, C. D. Vournas, G. K. Fourlas, "Hybrid systems modeling for power system," IEEE Circuits and Systems Magazine, vol. 4, no. 3, pp. 16-23, Third Quarter 2004.

[46] M. Negnevitsky, M.E. Haque, A. Gargoom, A.M.O. Haruni, "A Novel Operation and Control Strategy for a Standalone Hybrid Renewable Power System," IEEE Transactions on Sustainable Energy, vol. 4, no. 2, pp. 402-413, April 2013. 
[47] M. Prodanovic, T.C. Green, N. Pogaku, "Modeling, Analysis and Testing of Autonomous Operation of an Inverter-Based Microgrid," IEEE Transactions on Power Electronics, vol. 22, no. 2, pp. 613-625, March 2007.

[48] M. R. Iravani, F. Katiraei, "Power Management Strategies for a Microgrid With Multiple Distributed Generation Units," IEEE Transactions on Power Systems, vol. 21, no. 4, pp. 1821-1831, November 2006.

[49] T. Logenthiran, D. Srinivasan, A.M. Khambadkone, Htay Nwe Aung, "Multiagent System for Real-Time Operation of a Microgrid in Real-Time Digital Simulator," IEEE Transactions on Smart Grid, vol. 3, no. 2, pp. 925-933, June 2012.

[50] Ding Li, Yi Kang Chai, F. Blaabjerg, P.C. Loh, "Autonomous Operation of Hybrid Microgrid With AC and DC Subgrids," IEEE Transactions on Power Electronics, vol. 28, no. 5, pp. 2214-2223, May 2013.

[51] Y. F. Cao, Z. Li, X. Cai G. Shi, "Impact of wind-battery hybrid generation on isolated power system stability," International Symposium on Power Electronics Electrical Drives Automation and Motion, pp. 757-761, June 2010.

[52] Wei Jiang and B. Fahimi, "Active Current Sharing and Source Management in Fuel CellBattery Hybrid ," IEEE Transactions on Industrial Electronics, vol. 57, no. 2, pp. 752-761, February 2010.

[53] Jin-Hong Jeon, Chang-Hee Cho, Jong-Bo Ahn, Sae-Hyuk Kwon, Seul-Ki Kim, "Dynamic Modeling and Control of a Grid-Connected Hybrid Generation System With Versatile Power Transfer," IEEE Transactions on Industrial Electronics, vol. 55, no. 4, pp. 1677-1688, April 2008.

[54] A.M. Osheiba, M.M. Khater, E.S. Abdin, "Modeling and optimal controllers design for a stand-alone photovoltaic-diesel generating unit," Energy Conversion, IEEE Transactions on, vol. 14, no. 3, pp. 560-565, September 1999.

[55] Jin-Hong Jeon, Seul-Ki Kim, Changhee Cho, June Ho Park, Hak-Man Kim, Kee-Young Nam, Jong-Yul Kim, "Cooperative control strategy of Energy Storage System and Microsources for Stabilizing the Microgrid during Islanded Operation," IEEE Transactions on Power Electronics, vol. 25, no. 12, pp. 3037-3048, December 2010.

[56] Jae-Jin Seo, Yun-Seong Kim, Dong-Jun Won, Loc Nguyen Khanh, "Power-Management Strategies for a Grid-Connected PV-FC Hybrid System," IEEE Transactions on Power Delivery, vol. 25, no. 3, pp. 1874-1882, July 2010. 
[57] Y.A.R.I. Mohamed, A.A.A. Radwan, "Modeling, Analysis, and Stabilization of ConverterFed AC Microgrids With High Penetration of Converter-Interfaced Loads," IEEE Transactions on Smart Grid, vol. 3, no. 3, pp. 1213-1225, September 2012.

[58] Jong-Yul Kim, Hak-Man Kim, Seul-Ki Kim, Changhee Cho, Jan-Mok Kim, Jong-Bo Ahn, KeeYoung Nam Jin-Hong Jeon, "Development of Hardware In-the-Loop Simulation System for Testing Operation and Control Functions of Microgrid," IEEE Transactions on Power Electronics, vol. 25, no. 12, pp. 2919-2929, December 2010.

[59] A. Alimardani, S.H. Hosseinian, M. Abedi, P.H. Divshali, "Decentralized Cooperative Control Strategy of Microsources for Stabilizing Autonomous VSC-Based Microgrids," IEEE Transactions on Power Systems, vol. 27, no. 4, pp. 1949-1959, November 2012.

[60] Di Lu H. Fakham and B. Francois, "Power Control Design of a Battery Charger in a Hybrid Active PV Generator for Load-Following Applications," IEEE Transactions on Industrial Electronics, vol. 58, no. 1, pp. 85-94, January 2011.

[61] T. SenjYu, A. Yona, T. Funabashi, Chul-Hwan Kim, M. Datta, "A Frequency-Contrrol Approach by Photovoltaic Generator in a PV-Diesel Hybrid Power System," IEEE Transactions on Energy Conversion, vol. 26, no. 2, pp. 559-571, June 2011.

[62] D.M. Divan, R. Adapa, M.C. Chandorkar, "Control of parrallel connected inverters in standalone AC supply systems," IEEE Transactions on Industry Applications, vol. 29, no. 1, pp. 136-143, January 1993.

[63] N. Mithulananthan, R.C. Bansal, T.K. Saha, A. Arulampalam, "Micro-grid control of PVWind-Disel hybird with islanded and grid connected operations," IEEE International Conference on Sustainable Energy Technologies, pp. 1-9, December 2010.

[64] Yung-Ruei Chang, Che-Wei Chang, "Energy storage systems for seamless mode transfer in microgrid," IEEE Ninth International Conference on Power Electronics and Drive Systems, pp. 799-802, December 2011.

[65] A. Petterteig, R. Pittini, T.M. Undeland S. D'Arco, "Droop regulated VSCs for island operation of future offshore systems," IEEE Trondheim Power Tech, pp. 19-23, June 2011.

[66] K.M. Muttaqi, S. Sayeef, S. Perera, N. Mendis, "Standalone Operation of Wind TurbineBased Variable Speed Generators With Maximum Power Extraction Capability," IEEE Transactions on Energy Conversion, vol. 27, no. 4, pp. 822-834, December 2012. 
[67] J.A. Ferreira, P. Bauer, Y. Zhou, "Grid-connected and islanded operation of a hybrid power system," IEEE Power Engineering Society Conference and Exposition in Africa, pp. 16-20, July 2007.

[68] S. Palle, Y. Sozer, I. Husain, M.N. Arafat, "Transition Control Strategy Between Standalone and Grid-connected Operations of Voltage-Source Inversters," IEEE Transactions on Industry Application, vol. 48, no. 5, pp. 1516-1525, October 2012.

[69] R.C.Bansal, "Automatic Reactive-Power Control of Isolated Wind/Diesel Hybrid Power Systems," IEEE Transactions on Industrial Electronics, vol. 53, no. 4, pp. 1116-1126, June 2006.

[70] B. Singh, S.S. Murthy, N. Kishore, P.K. Goel, "Isolated Wind-Hydro Hybrid System Using Cage Generators and Battery Storage," IEEE Transactions on Industrial Electronics, vol. 58, no. 4, pp. 1141-1153, April 2011.

[71] T. Nakaji, K. Uezato, T. Funabashi, T. Senjyu, "A hybrid power system using alternative energy facilities in isolated island," IEEE Transactions on Energy Conversion, vol. 20, no. 2, pp. 406-414, June 2005.

[72] T. Senjyu, A. Yona, T. Funabashi, Chul-Hwan Kim M. Datta, "A Coordinated Control Method for leveling PV Output Power Flucturations of PV-Diesel Hybrid System Connected to Isolated Power Utility," IEEE Transactions on Energy Conversion, vol. 24, no. 1, pp. 153-162, March 2009.

[73] M.R. Iravani, M.S. Khan, "Hybrid Control of a Grid-Interactive Wind Energy Conversion System," IEEE Transactions on Energy Conversion, vol. 23, no. 3, pp. 895-902, September 2008.

[74] K. Iba, "Reactive power optimization by genetic algorithm," IEEE Transactions on Power Systems, vol. 9, no. 2, pp. 685-692, May 1994.

[75] S.N. Deepa S.N. Sivanandam, Introduction to Genetic Algorithms.

[76] A.L.F. De Almeida, F. R.P. Cavalcanti M.O. Binelo, "MIMO Array Capacity Optimization Using a Genetic Algorithm," IEEE Transactions on Vehicular Technology, vol. 60, no. 6, pp. 2471-2481, July 2011.

[77] Darrel Whitley, A Genetic Algorithm Tutorial. 
[78] G. Ortiz, J.I. Yuz, J. Rodriguez, S. Vazquez, L.G. Franquelo, P. Cortes, "Model Predictive Control of an Inverter With Output LC Filter for UPS Applications," IEEE Transactions on Industrial Electronics, vol. 56, no. 6, pp. 1875-1883, June 2009.

[79] R. Boel, L. Vandevelde, M. Moradzadeh, "Voltage Coordination in Multi-Area Power Systems via Distributed Model Predictive Control," IEEE Transactions on Power Systems, vol. 28, no. 1, pp. 513-521, February 2013.

[80] J.A. Riveros, F. Barrero, H. Guzman, J. Prieto, M.J. Duran, "Reduction of Common-Mode Voltage in Five-Phase Induction Motor Drives Using Predictive Control Techniques," IEEE Transactions on Industry Applications, vol. 48, no. 6, pp. 2059-2067, November 2012.

[81] M. Saeedifard, Jiangchao Qin, "Predictive Control of a Modular Multilevel Converter for a Back-to-Back HVDC System," IEEE Transactions on Power Dilivery, vol. 27, no. 3, pp. 15381547, July 2012.

[82] A.M. Sharaf R.M. Hilloowala, "A rule-based fuzzy logic controller for a PWM inverter in a standalone wind energy conversion scheme," IEEE Transactions on Industry Applications, vol. 32, no. 1, pp. 57-65, Jan/Feb 1996.

[83] Minwon Park, In-keun Yu, T. Murata, J. Tamura, M.H. Ali, "Improvement of WindGenerator Stability by Fuzzy-Logic-Controlled SMES," IEEE Transactions on Industry Applications, vol. 45, no. 3, pp. 1045-1051, May-June 2009.

[84] V. Courtecuisse, C. Saudemont, B. Robyns, Jacques Deuse, M. El Mokadem, "Fuzzy Logic Supervisor-Based Primary Frequency Control Experiments of a Variable-Speed Wind Generator," IEEE Transactions on Power Systems, vol. 24, no. 1, pp. 407-417, February 2009.

[85] G.S. Stavrakakis, E.F. Nogaret G.N. Kariniotakis, "Wind power forecasting using advanced neural networks models," IEEE Transactions on Energy Conversion, vol. 11, no. 4, pp. $762-$ 767, December 1996.

[86] Da Zhang, S.Y. Foo, Hui Li, "A Stochastic Digital Implementation of a Neural Network Controller for Small Wind Turbine Systems," IEEE Transactions on Power Electronics, vol. 21, no. 5, pp. 1502-1507, September 2006.

[87] D.C. Wunsch, E.A. O'Hair, M.G. Giesselmann, Shuhui Li, "Using neural networks to estimate wind turbine power generation," IEEE Transactions on Energy Conversion, vol. 16, no. 3, pp. 276-282, September 2001. 
[88] Wei Qiao, "integrated control of wind farms, FACTs devices and the power network using neural networks and adaptive critic designs," Goergia Tech., Altlanta, Goergia, Ph.D Dissertation 2008.

[89] S. Ray and G.K.Venayagamoorthy, "Wide-area signal-based optimal neurocontroller for a UPFC," IEEE Trans. Power Del., vol. 23, no. 3, pp. 1597-1605, July 2008.

[90] G.K. Venayagamoorthy, B. Chaudhuri, and R. Majumder, S. Ray, "Comparison of adaptive critic-based and classical wide-area controllers for power systems," IEEE Trans. Syst., vol. 38, no. 4, pp. 1002-1007, August 2008.

[91] G.K. Venayagamoorthy, and R.G. Harley S. Mohagheghi, "Optimal wide area controller and state predictor for a power system," IEEETrans. Power Syst., vol. 22, no. 2, pp. 693705, May 2007.

[92] R.G. Harley, G.K. Venayagamoorthy, Wei Qiao, "Coordinated Reactive Power Control of a Large Wind Farm and a STATCOM Using Heuristic Dynamic Programming," IEEE Transactions on Energy Conversion, vol. 24, no. 2, pp. 493-503, June 2009.

[93] G.K. Venayagamoorthy, R.G. Harley, Jiaqi Liang, "Wide-Area Measurement Based Dynamic Stochastic Optimal Power Flow Control for Smart Grids With High Variability and Uncertainty," IEEE Transactions on Smart Grid, vol. 3, no. 1, pp. 59-69, March 2012.

[94] G.K. Venayagamoorthy, Jiaqi Liang, R.G. Harley, D. Molina, "Intelligent Local Area Signals Based Damping of Power System Oscillations Using Virtual Generators and Approximate Dynamic Programming," IEEE Transactions on Smart Grid, vol. 4, no. 1, pp. 498-508, March 2013.

[95] R.G. Harley, Wei Qiao, "Indirect Adaptive External Neuro-Control for a Series Capacitive Reactance Compensator Based on a Voltage Source PWM Converter in Damping Power Oscillations," IEEE Transactions on Industrial Electronics, vol. 54, no. 1, pp. 77-85, February 2007.

[96] R.G. Harley, D.C. Wunsch, G.K. Venayagamoorthy, "Implementation of adaptive criticbased neurocontrollers for turbogenerators in a multimachine power system," IEEE Transactions on Neural Networks, vol. 14, no. 5, pp. 1047-1064, September 2003.

[97] IEEE PES Distribution System Analysis Subcommittee Radial Test Feeders. [Online]. http://ewh.ieee.org/soc/pes/dsacom/tesfeeders.html

[98] D. Muthumuni, S. Santoso, "Distribution System Analysi," in IEEE PES AGM, Montreal, Canada, 2006. 
[99] W.H. Kersting, Distribution System Modeling and Analysis.: CRC Press, 2002.

[100] Monitoba HVDC Research Center, EMTDC Transient Analysis of PSCAD Power System Simulation User's Guide. Winnipeg, Canada.

[101] Manitoba HVDC Research Centre, EMTDC/PSCAD Power System Simulation Software User's Manual, 3rd ed., 1998 release.

[102] Eung-Sang Kim Seul-Ki Kim, "PSCAD/EMTDC-Based Modeling and Analysis of a Gearless Variable Speed Wind Turbines," IEEE Transactions on Energy Conversion, vol. 22, no. 2, pp. 421-430, June 2007.

[103] O. Anaya-lara and E. Acha, "Modeling and analysis of custom power systems by PSCAD/EMTDC," IEEE Transactions on Power Delivery, vol. 17, no. 1, pp. 266-272, January 2002.

[104] S.W.H. deHaan, H. Polinder, and W.L. Kling, J.G. Slootweg, "General model for representing variable speed wind turbines in power system dynamics simulations," IEEE Transactions on Power Systems, vol. 18, no. 1, pp. 144-151, February 2003.

[105] H. Nakayama, M. Sanada, and Y. Takeda, S. Morimoto, "Sensorless Output Maximization Control for Variable-Speed Wind Generation System Using IPMSG," IEEE Transactions on Industry Applications, vol. 41, no. 1, p. 1, January/February 2005.

[106] L. Qu, and R.G. Harley, W. Qiao, "Control of IPM Synchronous Generator for Maximum Wind Power Generation Considering Magnetic Saturation," IEEE Transactions on Industry Applications, vol. 45, no. 3, pp. 1095-1105, May/June 2009.

[107] Se-Kyo Chung, "A phase tracking system for three system utility interface inverters," IEEE Transactions on Power Electronics, vol. 15, no. 3, pp. 431-438, May 2000.

[108] D.M. Divan, R. Adapa, M.C. Chandorkar, "Control of parallel connected inverters in standalone AC supply systems," IEEE Transactions on Industry Applications, vol. 29, no. 1, pp. 136-143, January/February 1993.

[109] S.K. Solanki, J. Solanki, Junbiao Han, "Coordinated Predictive Control of a Wind/Battery Microgrid System," IEEE Journal of Emerging and Selected Topics in Power Electronics, vol. 1, no. 4, pp. 296-305, December 2013.

[110] A. Davoudi, A. Bidram, "Hierarchical Struture of Microgrids Control System," IEEE Transactions on Smart Grid, vol. 3, no. 4, pp. 1963-1976, December 2012. 
[111] Chen Xi, He Zhihai, Z. Sun, "Adaptive Critic Design for Energy Minimization of Portable Video Communication Device," IEEE Transactions on Circuits and Systems for Video Technology, vol. 20, no. 1, pp. 27-37, January 2010.

[112] M.E. Baran, A.Q. Huang, S. Bhattacharya, L. Anderson, S. Teleke, "Control Strategies for Battery Energy Storage for Wind Farm Dispatching," IEEE Transactions on Energy Conversion, vol. 24, no. 3, pp. 725-732, September 2009.

[113] M.E. Baran, S. Bhattacharya, A.Q. Huang, S. Teleke, "Optimal Control of Battery Energy Storage for Wind Farm Dispatching," IEEE Transactions on Energy Conversion, vol. 25, no. 3, pp. 787-794, September 2010.

[114] Xiangjun Li, Dong Hui, and Xiaokang Lai, "Battery Energy Storage Station (BESS)-Based Smoothing Control of Photovoltaic (PV) and Wind Power Generation Fluctuations," IEEE Transactions on Sustainable Energy, vol. 4, no. 2, pp. 464-473, April 2013.

[115] Jiaqi Liang, "WIND ENERGY AND POWER SYSTEM INTERCONNECTION, CONTROL, AND OPERATION FOR HIGH PENETRATION OF WIND POWER," Georgia Institute of Technology, Georgia, Ph.D. Dissertation 2012.

[116] S.K. Solanki, J. Solanki, Junbiao Han, "Multi-objective control scheme to improve the performance of three-phase grid-connected PV generation," IEEE Power and Energy Society General Meeting, vol. 1, no. 6, pp. 22-26, July 2012.

[117] M.A. Abido, M.A. Hassan, "Optimal Design of Microgrids in Autonomous and GridConnected Modes Using Particle Swarm Optimization," IEEE Transactions on Power Electronics, vol. 26, no. 3, pp. 755-769, March 2011.

[118] S. Kouro, B.L. Rocca, R. Vargas, J. Rodriguez, J.I. Leon, S. Vazquez, and L.G. Franquelo, P. Cortes, "Guidelines for weighting factors design in model predictive control of power converters and drives," IEEE Int. Conf. Ind. Technol., pp. 1-7, 2009.

[119] IEEE PES Distribution Systems Analysis Subcommittee Radial Test Feeder. http://ewh.ieee.org/soc/pes/dsacom/tesfeeders.html.

[120] S, D. Muthumuni, S. Santoso, "Distribution System Analysis," in IEE PES AGM, Montreal, Canada, 2006. 


\section{Appendix A Distribution Network Modeling}

The time domain modeling of IEEE 13 node feeder consists of three elements, distribution line modeling, load modeling and regulator modeling. Models are developed for unbalanced distribution lines, unbalanced spot and distributed loads in different configurations, transformers and regulators.

\section{A.1 Distribution line modeling}

A mutually coupled model is used to model unbalanced distribution line which results in three by three impedance matrix. Table A.1 show the unbalance model of line 601,

TABle A.1. Distribution line model of line 601

\begin{tabular}{|c|c|c|c|c|c|c|c|}
\hline \multicolumn{2}{|c|}{$\begin{array}{c}\text { Resistance } \\
\text { (ohm) }\end{array}$} & \multicolumn{2}{c|}{$\begin{array}{c}\text { Reactance } \\
\text { (ohm) }\end{array}$} & \multicolumn{2}{c|}{$\begin{array}{c}\text { Mutual Resistance } \\
\text { (ohm) }\end{array}$} & \multicolumn{2}{c|}{$\begin{array}{c}\text { Mutual Resistance } \\
\text { (ohm) }\end{array}$} \\
\hline A & 0.3375 & A & 1.0478 & A-B & 0.156 & A-B & 0.5017 \\
\hline B & 0.3465 & B & 1.0179 & B-C & 0.158 & B-C & 0.4236 \\
\hline C & 0.3414 & C & 1.0348 & A-C & 0.1535 & A-C & 0.3849 \\
\hline
\end{tabular}

As seen in table A.1 the A-A, B-B and C-C elements form the three diagonal elements of the impedance matrix and $\mathrm{A}-\mathrm{B}, \mathrm{A}-\mathrm{C}$ and $\mathrm{B}-\mathrm{C}$ form the six off diagonal elements of the impedance matrix. For two phase line, one of the diagonal elements of the impedance matrix is zero. However, PSCAD does not accept zero as the input for the missing phase impedance. $0.000001 \mathrm{ohm}$ is then used as the resistance of the missing phase in line 603 and 604 to avoid singularity. Moreover, phases of bus 601, 602, 603 and 604 are transposed to implement the configuration of overhead lines as in data given in reference [101].

\section{A.2 Load modeling}

There are four most commonly used types of load models in distribution network such as distributed load, constant power load, constant current load and constant impedance load. The existing transmission module libraries as well as voltage stability load models in PSCAD 
are not suited for distribution systems due to the load characteristics matching the four models discussed above. The available distribution PQ load model in PSCAD does not give accurate results as it uses constant PQ load model to represent all kinds of load as shown in [98]. Approaches to model loads in PSCAD are given as below-

- As shown in Fig.A.1, the one third model presented in [99] is used where the total distributed load is lumped at one third line length

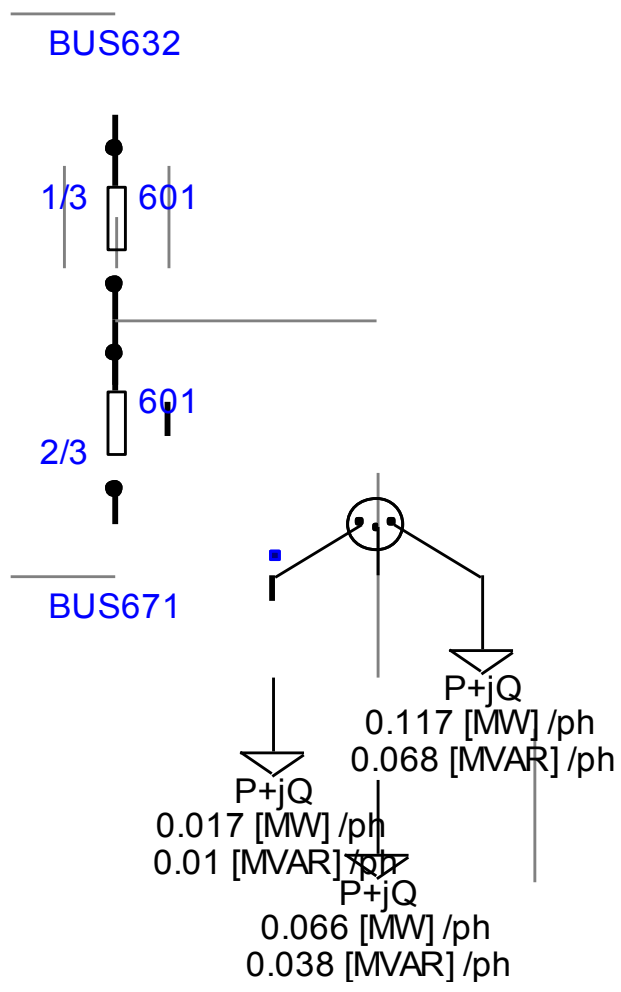

Fig.A.1 Distributed Load Modeling in PSCAD

- Loads are modeled as shunt connected combination of variable resistor and inductor.

Active and reactive powers are specified in the load data and, hence $L$ and $R$ need to be calculated according to the load type as seen in Table A.2 where $P$ and $Q$ are the active and reactive powers of load and $U$ is voltage.

\section{TABLE A.2}

LOAD IMPEDANCE CALCULATION

\begin{tabular}{|c|c|c|c|c|}
\hline Load type & \multicolumn{2}{|c|}{ Impedance } & First & Real time value \\
\cline { 2 - 3 } & $\mathrm{R}$ & $\mathrm{L}$ & iteration & \\
\hline Constant & $\frac{P}{U^{2}}$ & $\frac{Q}{2 \pi f U^{2}}$ & $\begin{array}{c}\text { P, Q, U are } \\
\text { rated }\end{array}$ & P=Rated, Q=Rated \\
Impedance & & & values & U=Rated \\
& & & & \\
\hline
\end{tabular}




\begin{tabular}{|c|c|c|c|c|}
\hline $\begin{array}{c}\text { Constant } \\
\text { Power }\end{array}$ & $\frac{P}{U^{2}}$ & $\frac{Q}{2 \pi f U^{\mathbf{2}}}$ & $\begin{array}{c}\mathrm{P}, \mathrm{Q}, \mathrm{U} \text { are } \\
\text { rated value }\end{array}$ & $\begin{array}{c}\mathrm{P}=\text { Rated, } \mathrm{Q}=\text { Rated } \\
\mathrm{U}=\text { Actual }\end{array}$ \\
\hline $\begin{array}{c}\text { Constant } \\
\text { Current }\end{array}$ & $\frac{U_{1} U_{2}}{P}$ & $\frac{U_{\mathbf{1}} U_{\mathbf{2}}}{\mathbf{2} \pi f Q}$ & $\begin{array}{c}\mathrm{P}, \mathrm{Q}, \mathrm{U} \text { are } \\
\text { rated value }\end{array}$ & $\begin{array}{c}\mathrm{P}=\text { Rated, } \mathrm{Q}=\text { Rated } \\
\mathrm{U} 1=\text { Actual,U2=Rated }\end{array}$ \\
\hline
\end{tabular}

The PSCAD implementation of various load types is shown in Fig.A.2 and Fig.A.3. P, Q and V are both constant in impedance load model while only P and Q are constant in constant PQ load as shown in Table II.

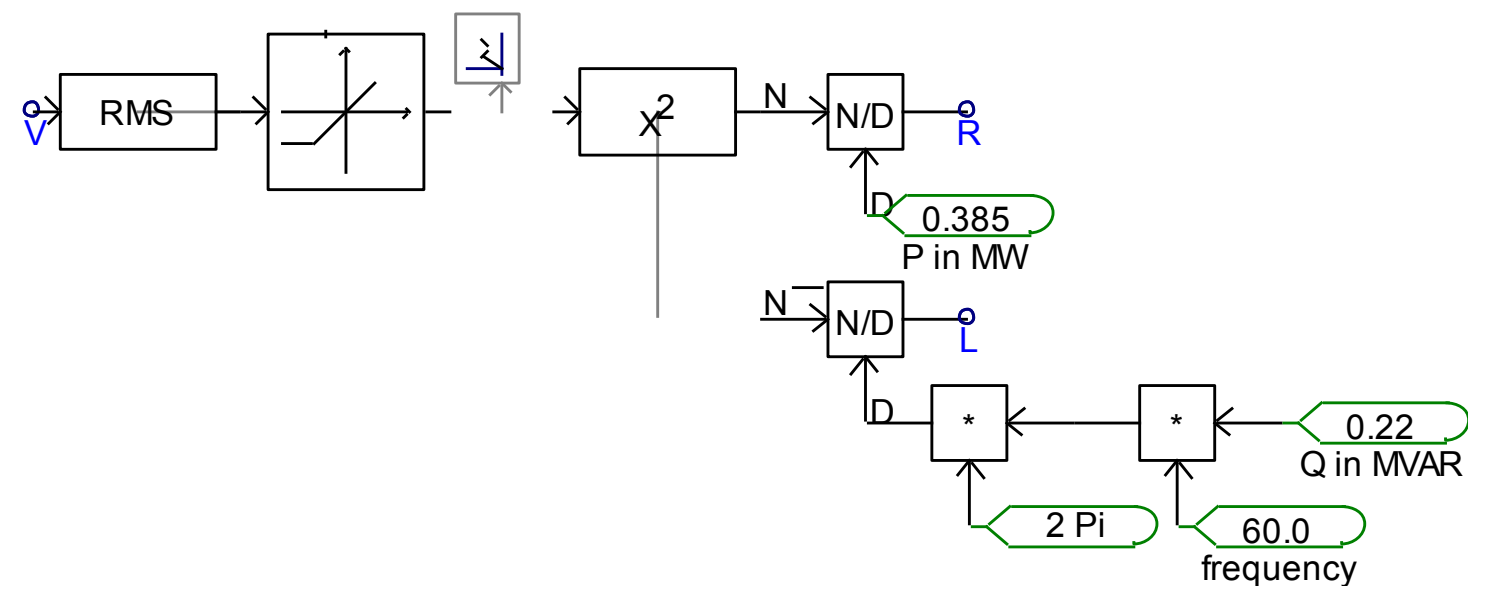

Fig.A.2 Constant Impedance and Constant Power Load
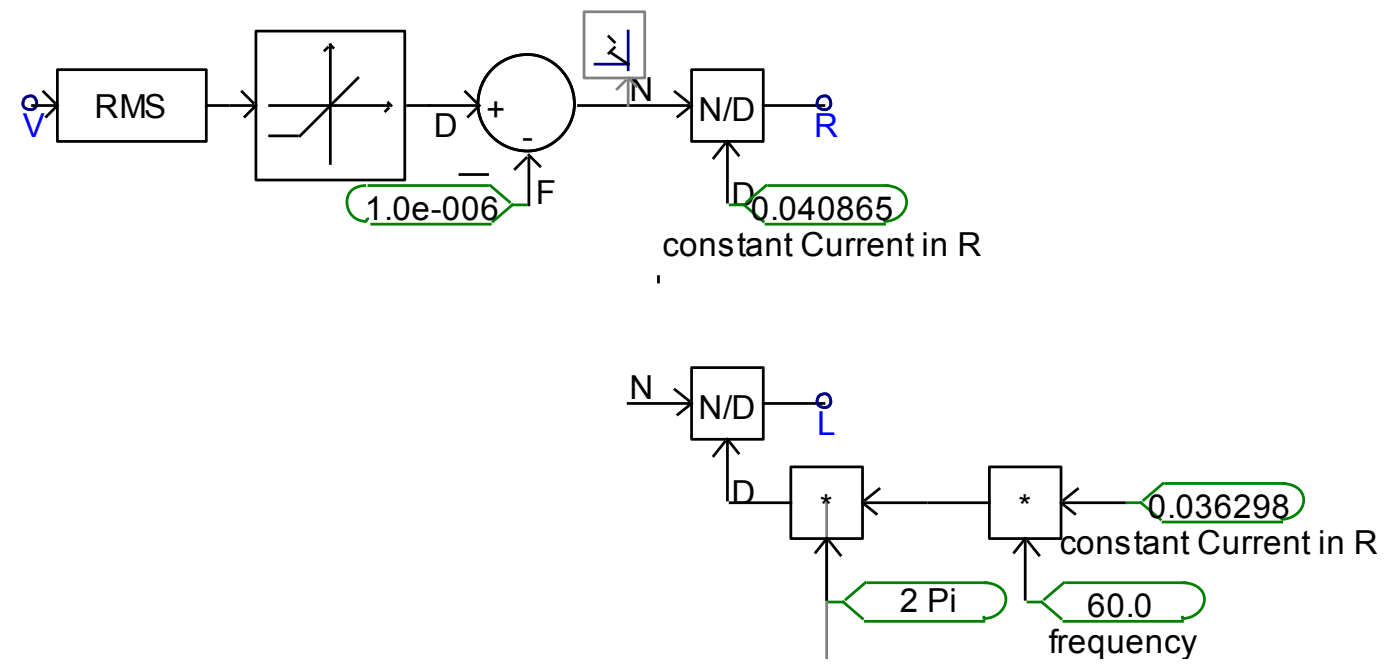

Fig.A.3 Constant Current Load

\section{A.3 Regulator modeling}

There are different types of regulator models and Type A and Type B are most commonly used in distribution networks. Here the regulator is modeled use the Type B line compensator 
model. Primary CT rating, CT series resistor R-VOLT, and CT series reactance X-VOLT are provided in the [101]. These values can be used to calculate, the actual CT rating, actual resistor and inductance required by PSCAD model as shown in equation (A.1-A.3),

$$
\begin{gathered}
\text { Actual CT Ratio }=\frac{\text { Primary CT Rating }}{\text { TurningRatio }} \\
R=\frac{R-\text { VOLT }}{\text { TurningRatio }} \\
L=\frac{L-\text { VOLT }}{\text { TurningRatio } \times 2 \pi f}
\end{gathered}
$$

\section{A.4 OpenDSS Implementation}

Using IEEE 13 node test feeder datasheet, different power system elements such as generators, lines, transformers, and loads have been modeled in OpenDSS software. The characteristics of components have been defined in ".dss" file. These components include voltage regulators, transformers, line impedances, loads and capacitors. For distributed loads, exact lumped load model has been used and an accurate full Carson method has been applied for calculating line impedances.

\section{A.5 Comparison between PSCAD and OpenDSS}

The steady state characteristics of the established electromagnetic transient IEEE 13 bus feeder are evaluated by comparing the power flow results with IEEE published results as shown in Table A.3. As seen in Table A.3 the maximum error in bus voltage magnitude is 0.069 per unit and maximum error in angle is 0.33 degrees respectively. These errors are due to the different power flow methodologies in different software.

\begin{tabular}{|c|c|c|c|c|c|c|c|c|c|c|c|c|c|c|}
\hline Node & MAG & ANGLE & MAG & ANGLE & MAG & ANGLE & MAG & ANGLE & MAG & ANGLE & MAG & ANGLE & \multicolumn{2}{|c|}{ Difference } \\
\hline & \multicolumn{2}{|c|}{ PSCAD } & \multicolumn{2}{|c|}{ OpenDSS } & \multicolumn{2}{|c|}{ PSCAD } & \multicolumn{2}{|c|}{ OpenDSS } & \multicolumn{2}{|c|}{ PSCAD } & \multicolumn{2}{|c|}{ OpenDSS } & $\begin{array}{l}\text { MAG } \\
(\mathrm{pu})\end{array}$ & $\begin{array}{c}\text { ANGLE } \\
\text { (deg) }\end{array}$ \\
\hline & \multicolumn{2}{|c|}{ A-N } & \multicolumn{2}{|c|}{ A-N } & \multicolumn{2}{|c|}{ B-N } & \multicolumn{2}{|c|}{ B-N } & \multicolumn{2}{|c|}{$\mathrm{C}-\mathrm{N}$} & \multicolumn{2}{|c|}{ C-N } & Max & Max \\
\hline 650 & 1.000 & 0.00 & 1.0000 & 0.00 & 1.000 & $\begin{array}{l}-120.00 \\
\end{array}$ & 1.0000 & -120.00 & 1.000 & 120.00 & 1.0000 & 120.00 & 0 & 0 \\
\hline RG60 & 1.062 & 0.00 & 1.0625 & 0.00 & 1.050 & -120.00 & 1.0500 & -120.00 & 1.069 & 120.00 & 1.0687 & 120.00 & 0.0005 & 0 \\
\hline 632 & 1.021 & -2.37 & 1.0210 & -2.49 & 1.037 & -121.53 & 1.0420 & -121.72 & 1.023 & 117.92 & 1.0174 & 117.83 & 0.005 & 0.12 \\
\hline 633 & 1.018 & $\begin{array}{l}-2.43 \\
\end{array}$ & 1.0180 & -2.56 & 1.035 & -121.56 & 1.0401 & $\begin{array}{l}-121.77 \\
\end{array}$ & 1.021 & 117.91 & 1.0148 & 117.82 & 0.0062 & 0.13 \\
\hline 634 & 0.994 & -3.09 & 0.9940 & -3.23 & 1.016 & -122.03 & 1.0218 & -122.22 & 1.001 & 117.44 & 0.9960 & 117.34 & 0.0042 & 0.14 \\
\hline 645 & & & & & 1.028 & -121.71 & 1.0329 & -121.90 & 1.021 & 117.95 & 1.0155 & 117.86 & 0.0049 & 0.19 \\
\hline 646 & & & & & 1.026 & \begin{tabular}{|l|}
-121.79 \\
\end{tabular} & 1.0311 & $\begin{array}{l}-121.98 \\
\end{array}$ & 1.019 & 117.99 & 1.0134 & 117.90 & 0.0045 & 0.19 \\
\hline 671 & 0.990 & -5.09 & 0.9900 & -5.30 & 1.046 & -122.02 & 1.0529 & -122.34 & 0.987 & 116.35 & 0.9778 & 116.02 & 0.0069 & 0.33 \\
\hline
\end{tabular}

TABLE A.3

IEEE 13 NODE POWER FLOW COMPARISON OF PSCAD WITH OPENDSS FEEDER 


\begin{tabular}{|c|c|c|c|c|c|c|c|c|c|c|c|c|c|c|}
\hline 680 & 0.990 & -5.09 & 9900 & $\begin{array}{l}-5.30 \\
\end{array}$ & 1.046 & -122.02 & 1.0529 & -122.34 & 0.987 & 116.35 & $\begin{array}{l}.9778 \\
\end{array}$ & 116.02 & 0.0069 & 0.33 \\
\hline 684 & 0.990 & -5.11 & 0.9881 & -5.32 & & & & & 0.986 & 116.34 & 0.9858 & 115.92 & 0.0002 & 0.42 \\
\hline 611 & & & & & & & & & 0.975 & 116.36 & 0.9738 & 115.78 & 0.0012 & 0.58 \\
\hline 652 & 0.988 & -5.08 & 0.9825 & -5.25 & & & & & & & & & 0.0055 & 0.17 \\
\hline 692 & 0.990 & -5.09 & 0.9900 & \begin{tabular}{|l|}
-5.31 \\
\end{tabular} & 1.046 & -122.02 & 1.0529 & -122.34 & 0.987 & 116.35 & 0.9877 & 116.02 & 0.0003 & 0.33 \\
\hline 675 & 0.984 & -5.33 & 0.9835 & -5.56 & 1.048 & -122.19 & 1.0553 & -122.52 & 0.985 & 116.36 & 0.9858 & 116.03 & 0.0002 & 0.33 \\
\hline
\end{tabular}

\section{A.6 IEEE WECC 9 bus system}

A modified IEEE WECC 9 bus system model is also created in PSCAD. The procedure is similar as IEEE 13 bus system. The active power, voltage amplitude and angle are exactly match, and the absolute maximum reactive power error is $0.2 \mathrm{Mvar}$. The power flow result is shown in Table A.4 and A.5.

TABLE A.4

BUS VOLTAGE OF POWER FLOW RESULT OF MODIFIED IEEE WECC 9 BUS

\begin{tabular}{|l|l|l|}
\hline Node number & $\begin{array}{l}\text { Voltage magnitude of Phase A } \\
\text { (p.u.) }\end{array}$ & Phase A Voltage angle $\left(^{\circ}\right.$ ) \\
\hline 1 & 1.000 & 0.000 \\
\hline 2 & 1.000 & 9.621 \\
\hline 3 & 1.000 & 4.745 \\
\hline 4 & 0.984 & -2.602 \\
\hline 5 & 0.966 & -4.514 \\
\hline 6 & 1.001 & 1.783 \\
\hline 7 & 0.984 & 0.496 \\
\hline 8 & 0.995 & 3.700 \\
\hline 9 & 0.955 & 4.532 \\
\hline
\end{tabular}

TABLE A.5

LINE FLOW OF POWER FLOW RESULT OF MODIFIED IEEE WECC 9 BUS

\begin{tabular}{|c|c|c|c|c|}
\hline \multirow[t]{2}{*}{ Branch } & \multicolumn{2}{|l|}{ IEEE } & \multicolumn{2}{|l|}{ PSCAD } \\
\hline & $\begin{array}{l}\text { Active Power } \\
\text { (MW) }\end{array}$ & $\begin{array}{l}\text { Reactive } \\
\text { Power(Mvar) }\end{array}$ & $\begin{array}{l}\text { Active Power } \\
\text { (MW) }\end{array}$ & $\begin{array}{l}\text { Reactive Power } \\
\text { (Mvar) }\end{array}$ \\
\hline 1 to 4 & 71.95 & 24.07 & 71.12 & 24.07 \\
\hline 4 to 5 & 30.73 & -0.59 & 30.83 & 0.71 \\
\hline 6 to 5 & 60.89 & -12.43 & 60.88 & -12.33 \\
\hline 3 to 6 & 85 & -3.65 & 85.07 & -3.656 \\
\hline 6 to 7 & 24.11 & 4.54 & 24.19 & 4.43 \\
\hline 8 to 7 & 76.50 & 0.26 & 76.48 & 0.33 \\
\hline
\end{tabular}




\begin{tabular}{|l|l|l|l|l|}
\hline 2 to 8 & 163 & 14.46 & 163 & 14.66 \\
\hline 8 to 9 & 86.50 & -2.53 & 86.46 & -2.38 \\
\hline 4 to 9 & 41.23 & 21.34 & 41.38 & 21.14 \\
\hline
\end{tabular}

